
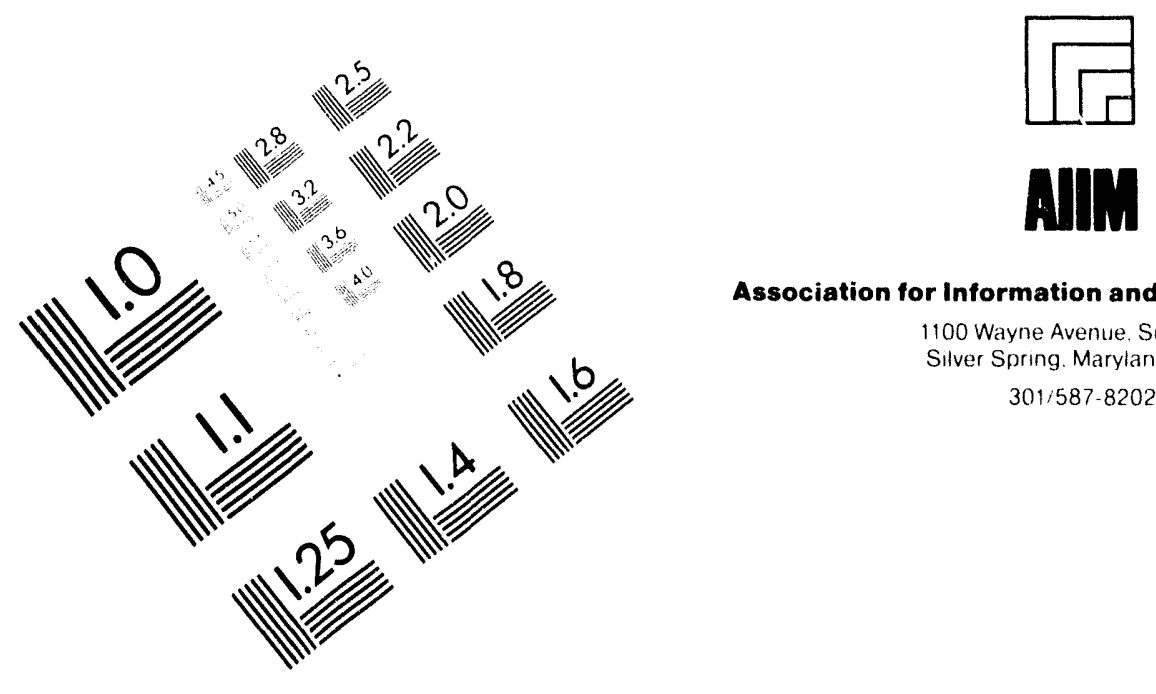

Association for Information and Image Management

1100 Wayne Avenue. Surte 1100

Silver Spring. Maryland 209 to

301/587-8202

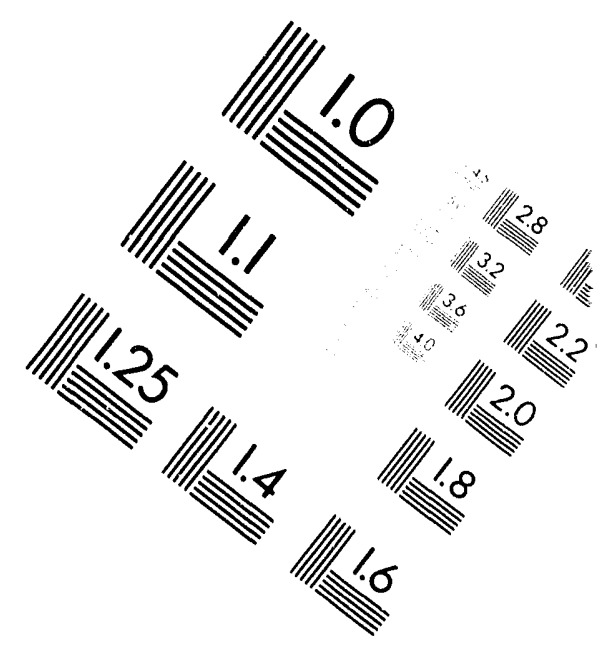

Centimeter

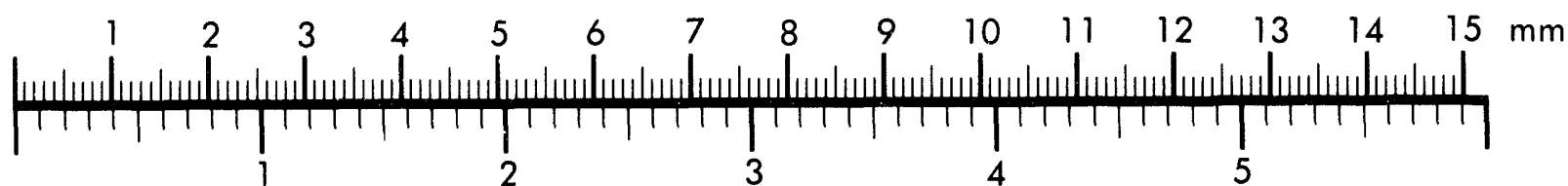

Inches

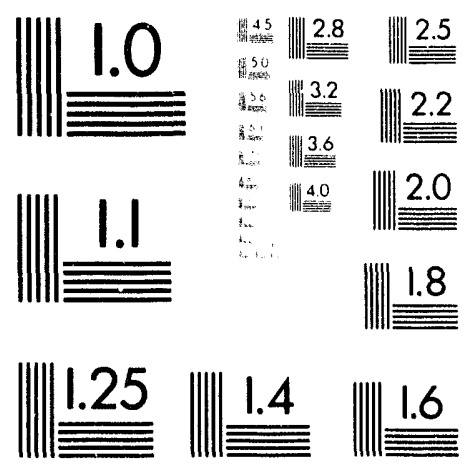

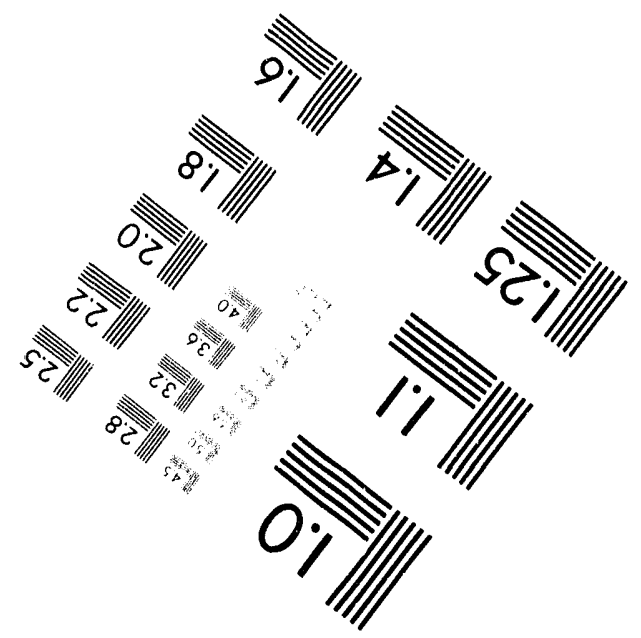

MANUFACTURED TO AIIM STANDARDS

BY APPLIED IMAGE, INC.

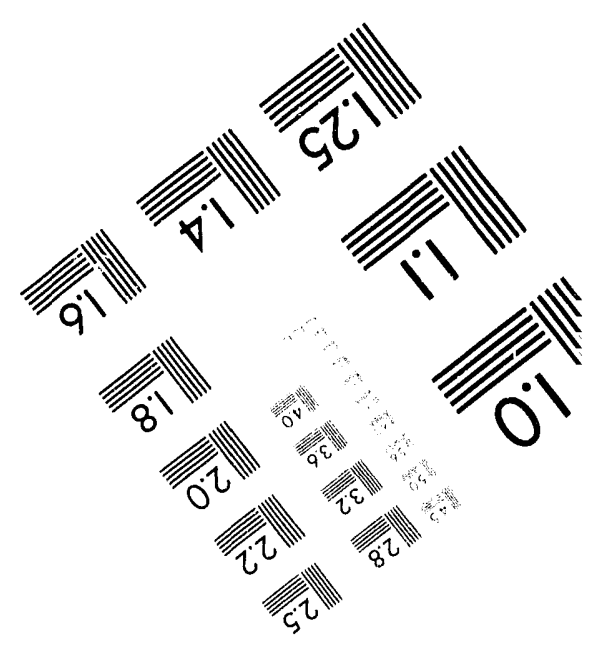



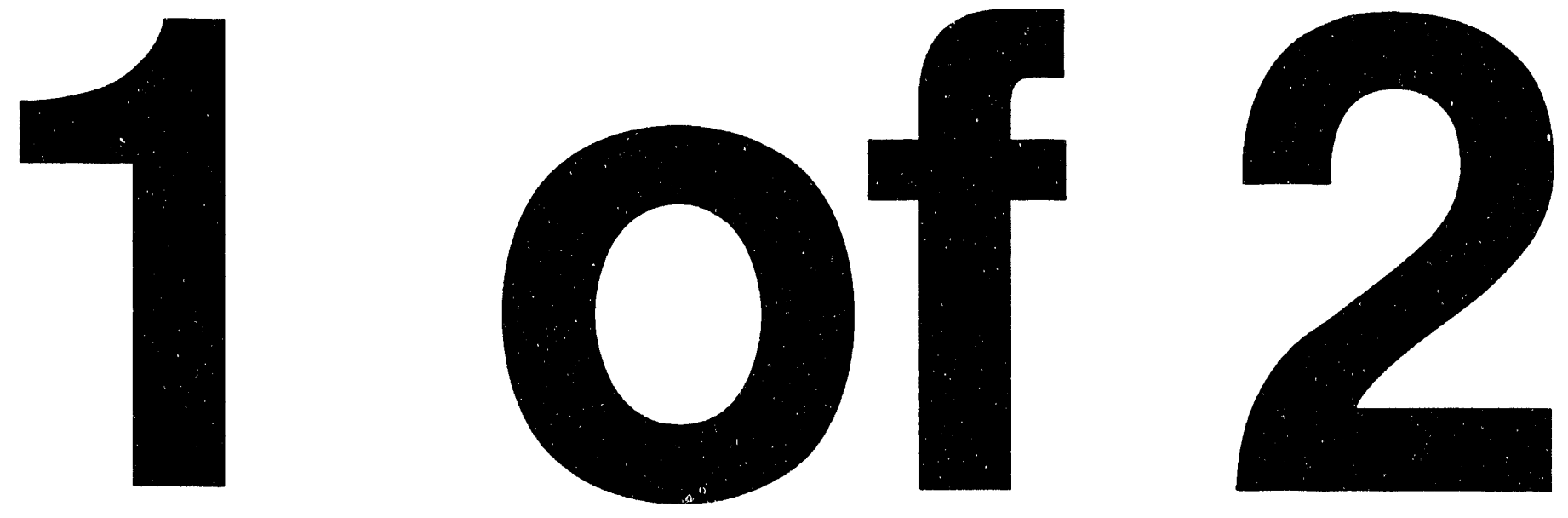


\section{OAK RIDGE} NATIONAL. LABORATORY

MAGTIN MAROETRA

\section{SECOND REPORT ON THE OAK RIDGE K-25 SITE BIOLOGICAL MONITORING AND ABATEMENT PROGRAM FOR MITCHELL BRANCH}

S. M. Adams

W. D. Crumby ${ }^{1}$

R. L. Hinzman

L. A. Kszos
J. G. Smith

J. M. Loar

M. J. Peterson

M. G. Ryon

G. R. Southworth

\footnotetext{
'Automated Sciences Group, Inc., Oak Ridge
}

Environmental Sciences Division

Publication No. 3928

March 1994

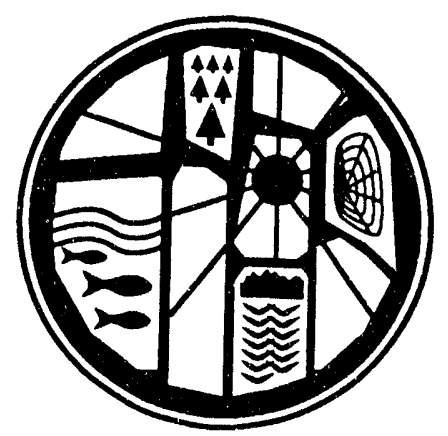


This report has ben reproduced directly from the best available copy.

Avallable to DOE and DOE contractors from the Office of Scientific and Technical Information, P.O. Box 62, Oak Ridge, TN 37831; prices available from (615) 576-8401, FTS 626-8401.

Availabte to the public from the National Technical Information Service, U.S. Department of Commerce, 5285 Port Royal Rd., Springfield, VA 22161.

This report was prepared as an account of work sponsored by an agency of the United States Government. Neither the United States Government nor any agency thereof, nor any of their employees, makes any warranty, express or implied, or assumes any legal liability or responsibility for the accuracy, completeness, or usefulness of any information, apparatus, product, or process disclosed, or represents that its use would not infringe privately owned rights. Reference herein to any specific commercial product, process, or service by trade name, trademark, manufacturer, or otherwise, does not necessarily constitute or imply its endorsement, recommendation, or favoring by the United States Government or any agency thereof. The views and opinions of authors expressed herein do not necessarily state or reflect those of the United States Government or any agency thereot. 
ENVIRONMENTAL SCIENCES DIVISION

\title{
SECOND REPORT ON THE OAK RIDGE K-25 SITE \\ BIOLOGICAL MONITORING AND ABATEMENT PROGRAM FOR MITCHELL BRANCH
}

\author{
J. G. Smith, Editor
}
S. M. Adams
W. D. Crumby ${ }^{1}$
J. M. Loar
R. L. Hinzman
M. J. Peterson
L. A. Kszos
M. G. Ryon
G. R. Southworth

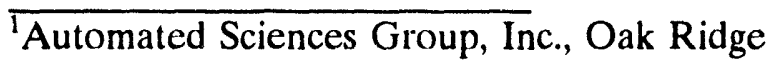

Environmental Sciences Division

Publication No. 3928

Manuscript Completed: December 1993

Date Published: March 1994

Prepared for

R. A. Rich

Water Quality and Hazardous Substances Department

Environmental Management Division

Oak Ridge K-25 Site

Oak Ridge, Tennessee 37831

Prepared by the

OAK RIDGE NATIONAL LABORATORY

Oak Ridge, Tennessee 37831-6285 managed by

MARTIN MARIETTA ENERGY SYSTEMS, INC.

for the

U.S. DEPARTMENT OF ENERGY

under contract No. DE-AC05-840R21400 


\section{CONTENTS}

Page

LIST OF FIGURES $\ldots \ldots \ldots \ldots \ldots \ldots \ldots \ldots \ldots \ldots \ldots \ldots \ldots \ldots \ldots \ldots \ldots \ldots \ldots$

LIST OF TABLES $\ldots \ldots \ldots \ldots \ldots \ldots \ldots \ldots \ldots \ldots \ldots \ldots \ldots \ldots \ldots \ldots \ldots \ldots$

ACRONYMS AND INITIALISMS $\ldots \ldots \ldots \ldots \ldots \ldots \ldots \ldots \ldots \ldots \ldots \ldots \ldots \ldots$ xvii

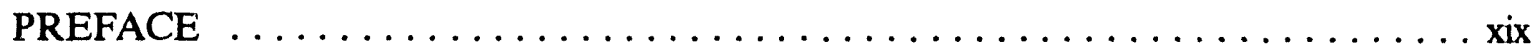

ACKNOWLEDGMENTS $\ldots \ldots \ldots \ldots \ldots \ldots \ldots \ldots \ldots \ldots \ldots \ldots \ldots \ldots \ldots \ldots \ldots \ldots$

EXECUTIVE SUMMARY $\ldots \ldots \ldots \ldots \ldots \ldots \ldots \ldots \ldots \ldots \ldots \ldots \ldots \ldots \ldots \ldots \ldots \ldots$

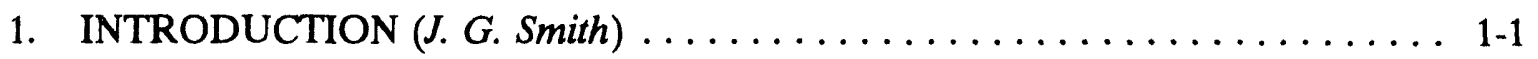

2. DESCRIPTION OF STUDY AREA (R. L. Hinzman and J. M. Loar) . . . . . 2-1

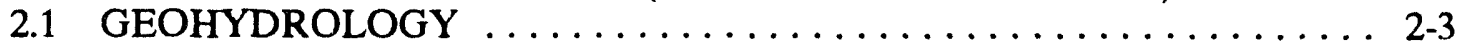

2.1.1 Mitchell Branch ............................ 2-3

2.1.2 Poplar Creek $\ldots \ldots \ldots \ldots \ldots \ldots \ldots \ldots \ldots \ldots \ldots \ldots \ldots \ldots \ldots \ldots \ldots, 2-6$

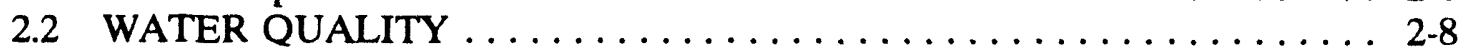

2.2.1 Description of the Oak Ridge

K-25 Site Discharges ...................... 2-8

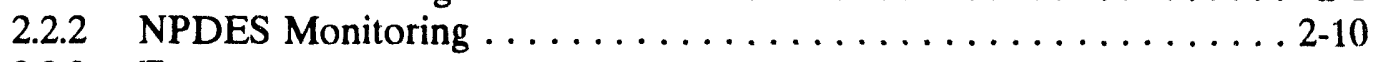

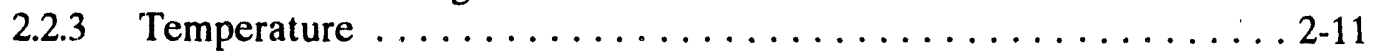

2.3 BIOLOGICAL MONITORING SITES $\ldots \ldots \ldots \ldots \ldots \ldots \ldots \ldots \ldots \ldots \ldots \ldots \ldots \ldots, 12$

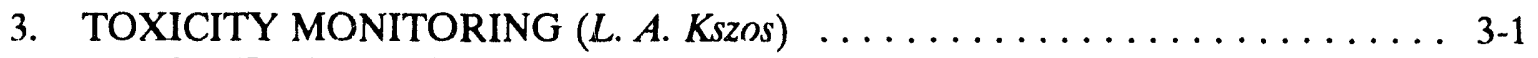

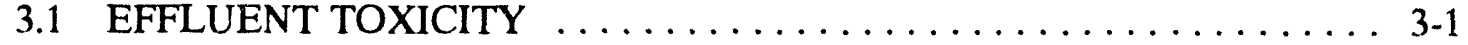

3.1 .1 Introduction $\ldots \ldots \ldots \ldots \ldots \ldots \ldots \ldots \ldots \ldots \ldots \ldots \ldots \ldots, 3-1$

3.1.2 Materials and Methods $\ldots \ldots \ldots \ldots \ldots \ldots \ldots \ldots \ldots \ldots, \ldots \ldots \ldots, 2$

3.1.3 Results $\ldots \ldots \ldots \ldots \ldots \ldots \ldots \ldots \ldots \ldots \ldots \ldots, \ldots, 2$

3.1.4 Discussion ................................ 3-15

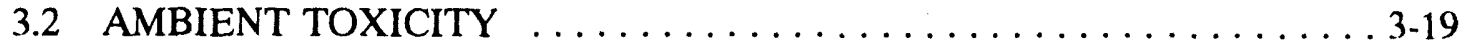

3.2.1 Introduction $\ldots \ldots \ldots \ldots \ldots \ldots \ldots \ldots \ldots \ldots \ldots \ldots \ldots \ldots \ldots \ldots \ldots, 19$

3.2.2 Materials and Methods $\ldots \ldots \ldots \ldots \ldots \ldots \ldots \ldots \ldots \ldots \ldots, 3-19$

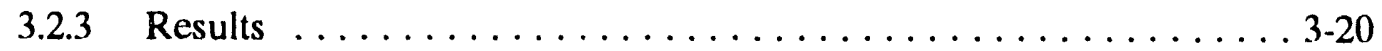

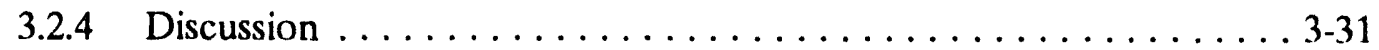

3.3 FUTURE STUDIES $\ldots \ldots \ldots \ldots \ldots \ldots \ldots \ldots \ldots \ldots \ldots \ldots \ldots \ldots \ldots \ldots \ldots, 33$ 
4. BIOACCUMULATION STUdIES (M. J. Peterson and G. R. Southworth) . . . 4-1

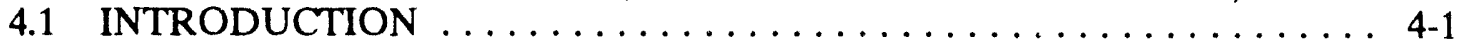

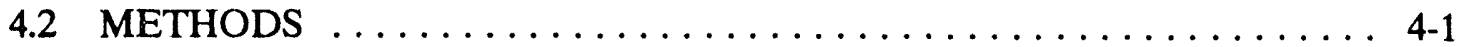

4.3 RESULTS AND DISCUSSION $\ldots \ldots \ldots \ldots \ldots \ldots \ldots \ldots \ldots \ldots$

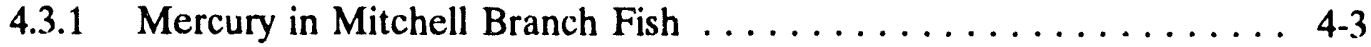

4.3.2 Mercury in Poplar Creek Fish ... . . . . . . . . . . . . . 4-5

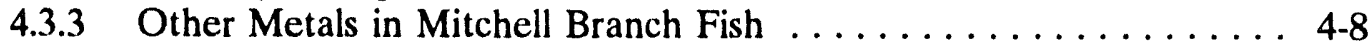

4.3.4 PCBs in Mitchell Branch Fish . . . . . . . . . . . . . . . 4-10

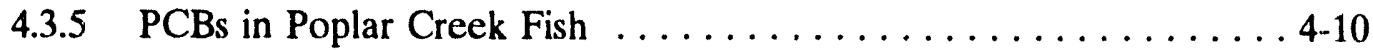

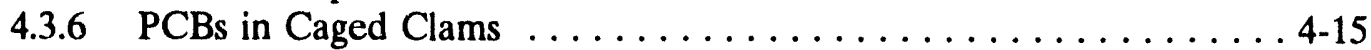

4.3.7 Other Organics in Caged Clams . . . . . . . . . . . . . . . 4-15

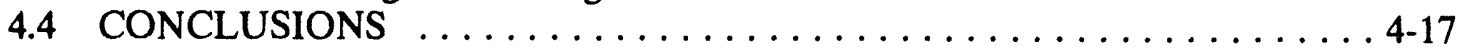

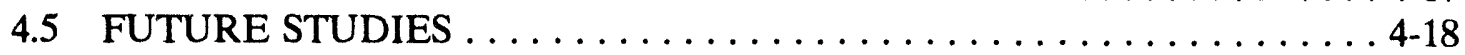

5. BIOLOGICAL INDICATORS OF CONTAMINANT-RELATED

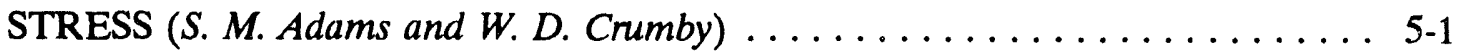

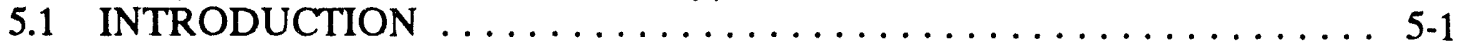

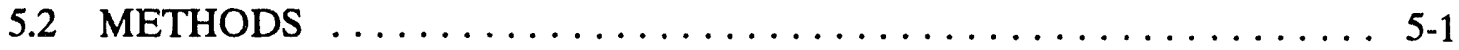

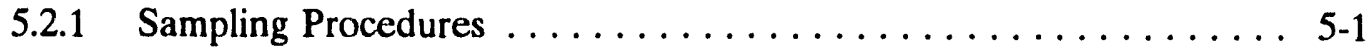

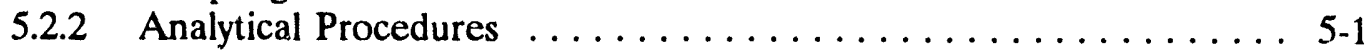

5.2 .3 Statistical Procedures . . . . . . . . . . . . . . . . . . . 5-4

5.3 RESULTS AND DISCUSSION $\ldots \ldots \ldots \ldots \ldots \ldots \ldots \ldots \ldots \ldots$

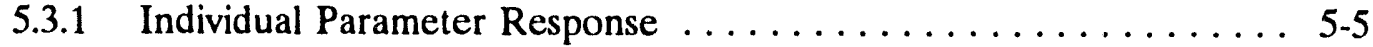

5.3.2 Integrated Bioindicator Analysis . . . . . . . . . . . . 5-11

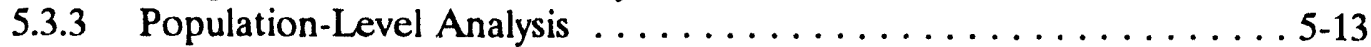

5.3.4 Summary and Synthesis . . . . . . . . . . . . . . . . . 5-17

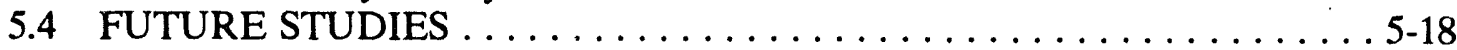

6. INSTREAM ECOLOGICAL MONITORING (J. G. Smith and M. G. Ryon) . . 6-1

6.1 BENTHIC MACROINVERTEBRATES (J. G. Smith) . . . . . . . . 6 6-1

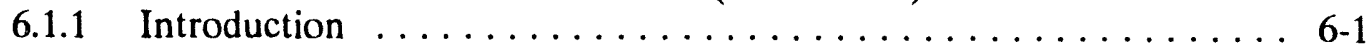

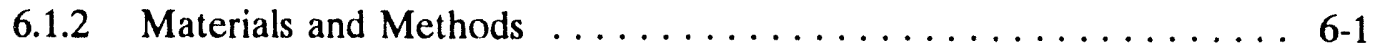

6.1 .3 Results ......................... 6-2

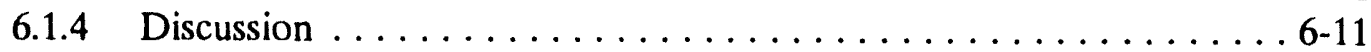

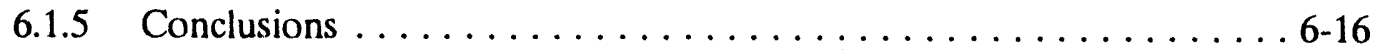

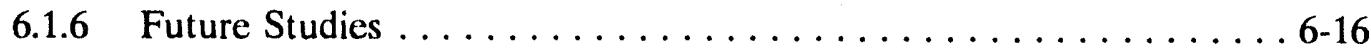

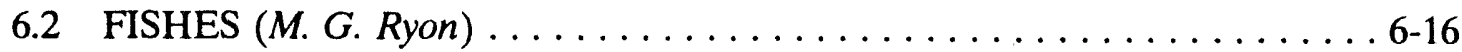

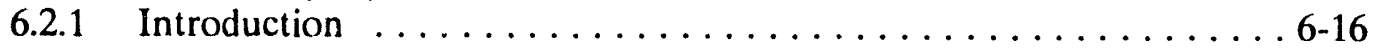

6.2 .2 Methods ............................. 6-17

6.2.3 Results and Discussion ..................... . 6-19

6.2 .4 Conclusions .......................... 6-28

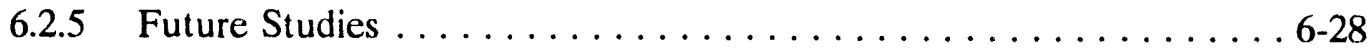




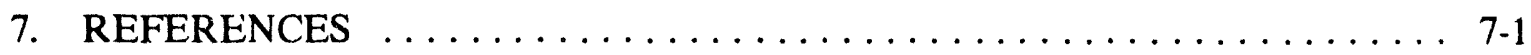

Appendix A. WATER QUALITY DATA FOR MITCHELL BRANCH $\ldots \ldots \ldots$ A-1

Appendix B. MEAN CONCENTRATIONS OF SELECTED

PARAMETERS IN MITCHELL BRANCH WATER

OBTAINED BY THE OAK RIDGE K-25 SITE

PROCESS SUPPORT DEPARTMENT IN CONJUNCTION

WITH THE TOXICITY TESTS CONDUCTED DURING

JULY 1988 THROUGH DECEMBER $1989 \ldots \ldots \ldots \ldots \ldots \ldots \ldots$. . . . .

Appendix C. RESULTS OF QUALITY ASSURANCE/QUALITY

CONTROL ANALYSES OF FISH SAMPLES $\ldots \ldots \ldots \ldots \ldots$.

Appendix D. CONCENTRATIONS OF MERCURY AND PCBs IN

INDIVIDUAL FISH FROM MITCHELL BRANCH,

POPLAR CREEK, AND NEARBY REACHES OF

THE CLINCH RIVER . . . . . . . . . . . . . . D 1

Appendix E. CHECKLIST OF BENTHIC MACROINVERTEBRATE

TAXA COLLECTED FROM MITCHELL BRANCH

AUGUST 1987 THROUGH JANUARY $1988 \ldots \ldots \ldots \ldots \ldots \ldots \ldots$ E-1 


\section{FIGURES}

Figure

Page

2.1 Map of Mitchell Branch and a portion of the Oak Ridge K-25 Site showing the locations of the biological monitoring sites in relation to the storm drains and the K-1407-B pond, which closed after October 31, 1988

2.2 Map of Mitchell Branch and a portion of the Oak Ridge K-25 Site showing the locations of the biological monitoring sites in relation to selected storm drains and the K-1407-E/F ponds and the K-1407-J basin, which replaced the K-1407-B pond on November 1, 1988

2.3 Map of the Oak Ridge area showing locations of the reference (control) sites ........................... 2-3

2.4 Mean monthly discharge in Poplar Creek $\ldots \ldots \ldots \ldots \ldots \ldots \ldots \ldots$

2.5 Diagram of effluent input into the Central Neutralization Facility

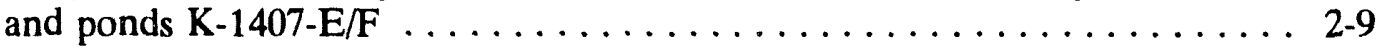

2.6 Weekly mean temperatures for Mitchell Branch kilometer 0.50 and Grassy Creek kilometer 2.40 for $1987-90 \ldots \ldots \ldots \ldots \ldots .12$

3.1 Annual mean conductivity, alkalinity, and hardness for each site in Mitchell Branch, 1987-90 . . . . . . . . . . . . . . . . . . 3-21

3.2 Cumulative frequency of total residual chlorine at 4 sites in Mitchell Branch for 22 tests, 1987-90

3.3 Cumulative frequency of total residual chlorine at Mitchell Branch kilometer (MIK) 0.12 and MIK $0.45,1987-90 \ldots \ldots \ldots \ldots$ 3-23

3.4 Cumulative frequency of total residual chlorine at Mitchell Branch kilometer (MIK) 0.54 and MIK $0.71,1987-90 \ldots \ldots \ldots \ldots . . \ldots \ldots .24$

3.5 Mean percentage survival and growth of fathead minnows for each

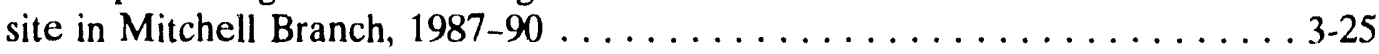

3.6 Frequency distribution of fathead minnow survival at Mitchell Branch kilometer (MIK) 0.12 and MIK 0.45, 1987-90 
Figure

3.7 Frequency distribution of fathead survival at Mitchell Branch kilometer (MIK) 0.54 and MIK $0.71,1987-90 \ldots \ldots \ldots \ldots \ldots \ldots \ldots$. . . . . . . . .

3.8 Frequency distribution of fathead survival at Mıchell Branch

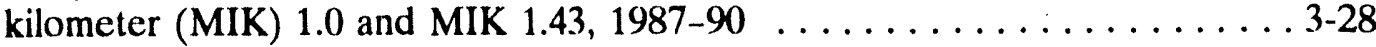

3.9 Mean survival and fecundity of Ceriodaphnia for each site in Mitchell Branch, 1987-90 . . . . . . . . . . . . . . . . . . . . . . . 3-29

3.10 Frequency distribution of Ceriodaphnia survival at Mitchell Branch kilometer (MIK) 0.12 and MIK $0.45,1987-90 \ldots \ldots \ldots \ldots \ldots$. . . . . . . . . . .

3.11 Frequency distribution of Ceriodaphnia survival at Mitchell Branch kilometer (MIK) 0.54 and MIK $0.71,1987-90 \ldots \ldots \ldots \ldots \ldots \ldots \ldots$. 3-31

3.12 Frequency distribution of Ceriodaphnia survival at Mitchell Branch

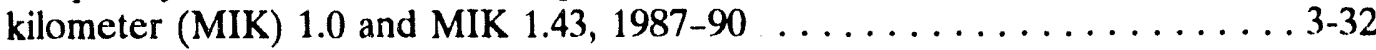

4.1 Mean concentrations of mercury in bluegill collected in fall/winter 1987 at sites on the Oak Ridge Reservation and nearby reaches of the Clinch River . . . . . . . . . . . . . . . . . . . . .

4.2 Mean concentrations of mercury in bluegill collected in fall/winter 1988 at sites on the Oak Ridge Reservation and nearby reaches of the Clinch River . . . . . . . . . . . . . . . . . . . . .

4.3 Mean concentrations of polychlorinated biphenyl in bluegill collected in fall/winter 1987 at sites on the Oak Ridge Reservation and nearby reaches of the Clinch River

4.4 Mean concentrations of polychlorinated biphenyl in bluegill collected in fall/winter 1988 at sites on the Oak Ridge Reservation and nearby reaches of the Clinch River

5.1 Levels of biological response in fish to contaminant stress, illustrating the continuum of these responses along gradients of

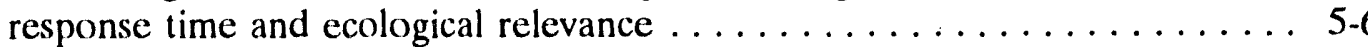

5.2 Relative differences in the response of detoxification enzymes for redbreast sunfish from each of the reference sites compared with Mitchell Branch 
Figure

5.3 Relative differences in the response of the organ dysfunction indicators for redbreast sunfish from each of the reference sites compared with Mitchell Branch

5.4 Relative differences in histopathological condition for redbreast sunfish from each of the reference sites compared with Mitchell Branch

5.5 Relative differences in the condition indices for redbreast sunfish from each of the reference sites compared with Mitchell Branch

5.6 Relative differences in the response of the nutritional indices for redbreast sunfish from each of the reference sites compared with Mitchell Branch . . . . . . . . . . . . . . . . . . . . . . . 5-11

5.7 Segregation of integrated health responses for redbreast sunfish from three reference sites and Mitchell Branch .

5.8 Length frequency of redbreast sunfish collected in Mitchell Branch, Hinds Creek, and Brushy Fork during August 1989

5.9 Mean total length of redbreast sunfish in each age group in August 1989 at the three sample sites

5.10 Mean weight of redbreast sunfish in each age group August 1989

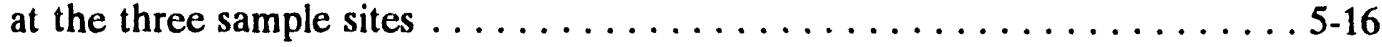

5.11 Mean instantaneous growth rate of redbreast sunfish in 1988 at the three sample sites

6.1 Mean monthly density of benthic macroinvertebrates in Mitchell

Branch, August 1987-January $1988 \ldots \ldots \ldots \ldots \ldots \ldots \ldots$ 6.5 . . . . . . . .

6.2 Mean monthly biomass of benthic macroinvertebrates in Mitchell

Branch, August $1987-\mathrm{January} 1988 \ldots \ldots \ldots \ldots \ldots \ldots \ldots$ 6.6. . . . . . .

6.3 Mean monthly taxonomic richness of benthic macroinvertebrates in Mitchell Branch, August 1987-January $1988 \ldots \ldots \ldots \ldots . . \ldots \ldots$. . . . . . . .

6.4 Mean monthly richness of Ephemeroptera, Plecoptera, and

Trichoptera in Mitchell Branch, August 1987-January 1988 $6-10$ 
Figure

Page

6.5 Mean monthly taxonomic diversity of benthic macroinvertebrates in Mitchell Branch, August 1987-January $1988 \ldots \ldots \ldots \ldots \ldots$. . . . . . . . . 6

6.6 Difference factor between each downstream site and the reference site and the magnitude of change within each site between Year 1 and Year 2 for mean density, biomass, taxonomic richness, and richness of Ephemeroptera, Plecoptera, and Trichoptera in Mitchell Branch.

A.1 Mean monthly streamflow for Mitchell Branch at Mitchell Branch kilometer 0.12 (National Pollutant Discharge Elimination System monitoring station K-1700) below Oak Ridge K-25 Site

A.2 Mean monthly discharge from pond K-1407-B for 1988 and from K-1407-E/F and K-1407-J for November 1988-December 1989 


\section{TABLES}

Table

Page

2.1 Comparison of mean annual discharge and percentage change between years for local streams with and without significant

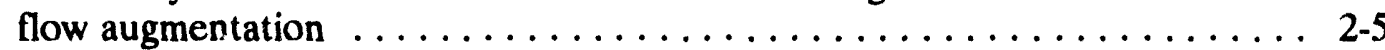

2.2 Number of days of zero discharge in upper Melton Branch at U.S. Geological Survey gaging station 03537100 near Oak Ridge National Laboratory

2.3 Monthly means and range of water temperature $\left({ }^{\circ} \mathrm{C}\right)$ at Mitchell Branch kilometer (MIK) 0.50 just below the outfall of storm drain 190 and at Grassy Creek kilometer (GCK) 2.4, a reference site ............................... 2-13

2.4 Location and description of the eight sites on Mitchell Branch that were sampled in various tasks of the Oak Ridge K-25 Site Biological Monitoring and Abatement Program ........... 2-16

3.1 Summary of toxicity test results and mean water quality parameters for effluent from the K-1407-B pond $\ldots \ldots \ldots \ldots \ldots$ 3-3

3.2 Summary of toxicity test results and mean water quality parameters for effluent from the $\mathrm{K}-1407-\mathrm{E} / \mathrm{F}$ ponds $\ldots \ldots \ldots \ldots \ldots . \ldots .4$

3.3 Mean concentrations of selected parameters in the effluents from the K-1407-E/F ponds obtained by the Oak Ridge K-25 Site Piocess Support Department in conjunction with the toxicity tests

3.4 Summary of toxicity tests results and mean water quality parameters for effluent from the K-1407-J basin

3.5 Mean concentrations of selected parameters for the K-1407-J basin effluent obtained by the Oak Ridge K-25 Site Process Support Department taken in conjunction with some of the toxicity tests

3.6 Summary of toxicity test results and concentration of total residual chlorine (TRC) in the storm drain effluents 
3.7 Mean concentrations of selected parameters in Storm Drain 170 effluent obtained by the Oak Ridge K-25 Site Process Support Department taken in conjunction with some of the toxicity tests

3.8 Mean concentrations of selected parameters in Storm Drain 180 effluent obtained by the Oak Ridge K-25 Site Process Support Department taken in conjunction with some of the toxicity tests . . . . . 3-14

3.9 Mean concentrations of selected parameters in Storm Drain 190 effluent obtained by the Oak Ridge K-25 Site Process Support Department taken in conjunction with some of the toxicity tests

3.10 Range in $\mathrm{pH}$ values at each site in Mitchell Branch for all tests

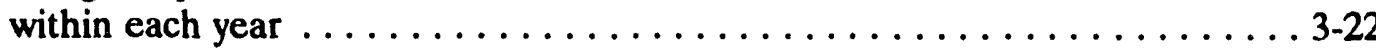

3.11 Percentage of fathead minnow tests at each site where no toxicity, acute toxicity, or chronic toxicity was observed

3.12 Percentage of Ceriodaphnia tests at each site where no toxicity, acute toxicity, or chronic toxicity was observed $\ldots \ldots \ldots \ldots \ldots \ldots \ldots \ldots .32$

4.1 Mercury concentrations in bluegill (Lepomis macrochirus) and redbreast sunfish ( $L$. auritus) collected at sites in the Poplar Creek drainage area in the vicinity of the Oak Ridge K-25 Site

4.2 Metal concentrations in redbreast sunfish (Lepomis auritus) collected from Mitchell Branch and Hinds Creek (reference stream)

4.3 PCB concentrations in bluegill (Lepomis macrochirus), redbreast sunfish ( $L$. auritus), channel catfish (Ictalurus punctatus), and gizzard shad (Dorosoma cepedianum) collected at sites in the Poplar Creek drainage area in the vicinity of the Oak Ridge K-25 Site

4.4 Concentrations of polychlorinated biphenyls (PCBs) and other organic contaminants in caged clams (Corbicula fluminea) maintained in Mitchell Branch for 4 weeks

4.5 Range of mean PCB concentrations measured in caged clams (Corbicula fluminea) at PCB-contaminated sites on Oak Ridge Reservation, $1988-90$. . . . . . . . . . . . . . . . . . . . . . . . 4-17 
5.1 Number of redbreast sunfish scale samples examined for each age

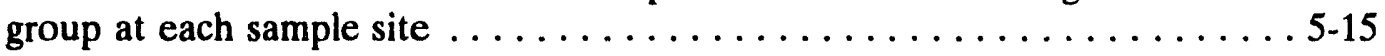

6.1 Benthic macroinvertebrate density, biomass, taxonomic richness, EPT richness, and taxonomic diversity in Mitchell Branch, August 1986-January 1987 and August 1987-January 1988

6.2 Statistical comparisons of mean benthic macroinvertebrate density, biomass, taxonomic richness (number of taxa), EPT richness, and taxonomic diversity for Mitchell Branch, August 1987-January 1988 . . . . . 6-4

6.3 Relative density and biomass of selected macroinvertebrate taxa in Mitchell Branch, August 1987-January $1988 \ldots \ldots \ldots \ldots \ldots$. . . . . . . 6-8

6.4 Length, mean width, mean depth, area, and pool/riffle ratio of fish sampling sites on Mitchell Branch and Grassy Creek, a reference stream, from January $1988-$ May $1990 \ldots \ldots \ldots \ldots$. . . . . . . . 6

6.5 Fish densities in Mitchell Branch and Grassy Creek, a reference stream, from January 1988-April/May $1990 \ldots \ldots \ldots \ldots \ldots$. . . . . . . . . 6-21

6.6 Fish biomass in Mitchell Branch and Grassy Creek, a reference

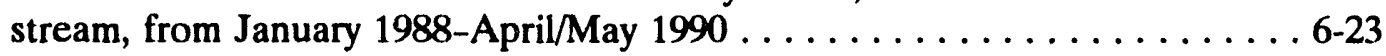

6.7 Annual production and production to biomass ratios, in parenthesis, in Mitchell Branch and Grassy Creek from spring 1987 to spring 1990

6.8 Annual production of fish communities in warm water streams in the Southeast

6.9 An analysis of variance comparison of fish condition factors between Mitchell Branch and Grassy Creek, January 1988-

April/May $1990 \ldots \ldots \ldots \ldots \ldots$. . . . . . . . . . . . . . . . . . . 6-27

A.1 Median and maximum concentration of National Pollutant Discharge Elimination System parameters in the water from lower Mitchell Branch

A.2 Median and maximum concentrations of organics routinely monitored in the water from lower Mitchell Branch 
A.3 Median and maximum concentrations of National Pollutant Discharge Elimination System parameters in the water from K-1407-B pond . . . . . . . . . . . . . . . . . .

A.4 Median and maximum concentrations of organics routinely monitored in the water from K-1407-B pond

A.5 Median and maximum concentrations of National Pollutant Discharge Elimination System parameters in the water from K-1407-E/F pond

A.6 Median and maximum concentrations of National Pollutant Discharge Elimination System parameters in the water from K-1407-J

A.7 Median and maximum concentrations of organics routinely monitored in the water from K-1407-J

A.8 Sampling frequency and sample type for regularly monitored water quality parameters as outlined in the National Pollutant Discharge Elimination System permit

B.1 Mean concentrations of selected parameters in Mitchell Branch water obtained by the Oak Ridge K-25 Site Process Support Department in conjunction with the toxicity test conducted during July 1988 .

B.2 Mean concentrations of selected parameters in Mitchell Branch water obtained by the Oak Ridge K-25 Site Process Support Department in conjunction with the toxicity test conducted during September $1988 \ldots \ldots \ldots \ldots \ldots \ldots \ldots \ldots \ldots \ldots \ldots$ B-

B.3 Mean concentrations of selected parameters in Mitchell Branch water obtained by the Oak Ridge K-25 Site Process Support Department in conjunction with the toxicity test conducted during November 1988 .

B.4 Mean concentrations of selected parameters in Mitchell Branch water obtained by the Oak Ridge K-25 Site Process Support Department in conjunction with the toxicity test conducted during January 1989 
Table

B.5 Mean concentrations of selected parameters in Mitchell Branch water obtained by the Oak Ridge K-25 Site Process Support Department in conjunction with the toxicity test conducted during March 1989

B.6 Mean concentrations of selected parameters in Mitchell Branch water obtained by the Oak Ridge K-25 Site Process Support Department in conjunction with the toxicity test conducted during May 1989

B.7 Mean concentrations of selected parameters in Mitchell Branch water obtained by the Oak Ridge K-25 Site Process Support Department in conjunction with the toxicity test conducted during July 1989

B.8 Mean concentrations of selected parameters in Mitchell Branch water obtained by the Oak Ridge K-25 Site Process Support Department in conjunction with the toxicity test conducted during September 1989

B.9 Mean concentrations of selected parameters in Mitchell Branch water obtained by the Oak Ridge K-25 Site Process Support Department in conjunction with the toxicity test conducted during December 1989

D.1 Concentrations of mercury and PCBs in resident fish from Mitchell Branch and nearby reaches of Poplar Creek and the Clinch River, $1987-90 \ldots \ldots \ldots \ldots \ldots \ldots$. . . . . . . . . . . . .

D.2 Detection limits of organic compounds $\ldots \ldots \ldots \ldots \ldots \ldots \ldots \ldots$

E.1 Checklist of benthic macroinvertebrate taxa collected from Mitchell Branch, August 1987 through Jariuary 1988 


\section{ACRONYMS AND INITIALISMS}

ACD

ALT

ANOVA

BCK

BMAP

BUN

CDFC

CNF

CRK

DEM

DO

DOE

EDTA

EFK

EFPC

EPA

EPT

ESD

FDA

GCK

GC/ECD

GC/MS

GLM

HESA

HSRD

LFPMA

LMACA

LOEC

LPARS

LSI

MAF

MHR

Analytical Chemistry Division

alanine aminotransferase

analysis of variance

Bear Creek kilometer

Biological Monitoring and Abatement Program

b'ood urea nitrogen

condition factor

Central Neutralization Facility

Clinch River kilometer

Department of Fnvironmental Management [name changed in 1987

to Department of Environmental Monitoring and Compliance

(DEMC)]

dissolved oxygen

U.S. Department of Energy

ethylenediaminetetracetic acid

East Fork Poplar Creek kilometer

East Fork Poplar Creek

U.S. Environmental Protection Agency

Ephemeroptera, Plecoptera, and Tricoptera

Environmental Sciences Division

U.S. Department of Agriculture Food and Drug Administration

Grassy Creek kilometer

gas chromatography/electron capture detector

gas chromatography/mass spectrometry

general linear model

Department of Health, Safety, and Environmental Affairs

Health and Safety Research Division

functional parenchyma in liver

macrophage aggregates in liver

lowest observed-effect concentration

liver parasites

liver-somatic index

mean annual flow

Melton Hill Reservoir 
MIK

$\mathrm{NAD}(\mathrm{H})$

NADP $(H)$

NOEC

NPDES

ORGDP

ORNL

ORR

PAH

PCB

PCK

PGV

PPM

SAS

SD

TDEC

TDS

TRC

TRK

TSCA

TSS

TVA

USGS

VSI

WCK

WOC
Mitchell Branch kilometer

nicotinamide-adenine dinucleotide (reduced form)

nicotinamide-adenine dinucleotide phosphate (reduced form)

no observed-effect concentration

National Pollutant Discharge Elimination System

Gak Ridge Gaseous Diffusion Plant

Oak Ridge National Laboratory

Oak Ridge Reservation

polycyclic aromatic hydrocarbons

polychlorinated biphenyl

Poplar Creek kilometer

preliminary guidance values

parts per million

Statistical Analysis System

storm drain

Tennessee Department of Environment and Conservation

total dissolved solids

total residual chlorine

Tennessee River kilometer

Toxic Substances Control Act

total suspended solids

Tennessee Valley Authority

U.S. Geological Survey

visceral-somatic index

White Oak Creek kilometer

White Oak Creek 


\section{PREFACE}

On September 11, 1986, a modified National Pollutant Discharge Elimination System permit was issued for the Oak Ridge Gaseous Diffusion Plant (ORGDP; now referred to as the Oak Ridge K-25 Site), a former uraniumenrichment production facility operated by Martin Marietta Energy Systems, Inc., for the U.S. Department of Energy. As required in Part III (L) of the permit, a plan for the biological monitoring of Mitchell Branch (K-1700 stream) was prepared and submitted for approval to the U.S. Environmental Protection Agency and the Tennessee Department of Environment and Conservation (Loar et al. 1992a). The plan, referred to as the
Oak Ridge K-25 Site Biological Monitoring and Abatement Program (BMAP), described biomonitoring activities that would be conducted over the duration of the permit.

This document is the second in a series of reports presenting the results of the studies that were conducted from August 1987 through June 1990 for the Oak Ridge K-25 Site BMAP. The actual period covered by each task or subtask varied, depending on when the task was initiated and the time needed for sample analysis and data review. These reports also address any significant modifications in the scope of work outlined in Loar et al. (1992a). 


\section{ACKNOWLEDGMENTS}

We thank D. K. Cox, W. M. Harris, W. C. Kyker, L. M. Stubbs, M. S. Greeley, S. L. Niemela, B. K. Beane, P. W. Braden, L. S. Ewald, G. P. Morris, J. Richmond, L. F. Wicker, and A. J. Stewart for their assistance in the laboratory an.\%/or field. We thank N. M. Ferguson, M. P. Maskarinec, T. B. Schope, and other staff of the Oak Ridge National Laboratory (ORNL) Analytical Chemistry Division for sample analyses. We thank B. F. Clark, W. C. Dickinson, A. W. McWhorter, and J. A. Wojtowicz of JAYCOR for processing the benthic macroinvertebrate samples;

\author{
W. C. Dickinson also reviewed an \\ earlier draft of Sect. 6.1. We are \\ grateful to G. F Cada and A. J. Stewart, \\ who reviewed a draft of this report and \\ provided many helpful suggestions and \\ comments. Finally, thanks to E. B. Bryant \\ for editorial support and L. J. Jennings \\ for electronic publishing of this document. \\ This work was funded by the Department \\ of Health, Safety, and Environmental \\ Affairs of the Oak Ridge K-25 Site. The \\ K-25 Site and ORNL are operated by \\ Martin Marietta Energy Systems, Inc., \\ under contract DE-AC05-840R21400 with \\ the U.S. Department of Energy.
}





\section{EXECUTIVE SUMMARY}

As a condition of the modified National Pollutant Discharge Elimination System (NPDES) permit issued to the Oak Ridge Gaseous Diffusion Plant (ORGDP; now referred to as the Oak Ridge K-25 Site) on September 11, 1986, a Biological Monitoring and Abatement Program (BMAP) was developed for the receiving stream (Mitchell Branch or $\mathrm{K}-1700$ stream). The objectives of BMAP are to (1) demonstrate that the effluent limitations established for the Oak Ridge K-25 Site protect and maintain the use of Mitchell Branch for growth and propagation of fish and other aquatic life and (2) document the effects on stream biota resulting from operation of major new pollution abatement facilities, including the Central Neutralization Facility (CNF) and the Toxic Substances Control Act (TSCA) incinerator. The BMAP consists of four tasks: (1) ambient toxicity testing; (2) bioaccumulation studies; (3) biological indicator studies; and (4) ecological surveys of stream communities, including benthic macroinvertebrates and fish. This document is the second in a series of reports on the results of the Oak Ridge K-25 Site BMAP; it describes studies that were conducted over various periods of time between August 1987 and June 1990.

\section{Background}

Mitchell Branch is a small stream that originates near the northeast boundary of the Oak Ridge K-25 Site; it flows only $1.5 \mathrm{~km}$ from its headwaters to its mouth at Poplar Creek kilometer 7.0. The water quality of Mitchell Branch is influenced by the geology of the drainage basin, effluents entering the stream via storm drains and the K-1407-E/F ponds, leachate from waste disposal sites, and remediation projects. The water quality of lower Mitchell Branch was characterized by (1) moderate levels of dissolved solids and occasionally high turbidity; (2) low concentrations of nitrogen relative to concentrations of phosphorus; (3) elevated levels of many metals and some organics; and (4) elevated temperatures. The geology of Mitchell Branch watershed contributes to periods of no flow in its upper reaches, whereas flow in its lower reaches is augmented by as much as $31 \%$ by discharges from the Oak Ridge K-25 Site. Samples for BMAP were routinely collected from eight primary sites in Mitchell Branch. The toxicity monitoring and community studies included at least five of the primary sites; four of the eight sites were common among all tasks. The site farthest upstream served as an undisturbed reference; the remaining sites were selected to coincide with the ambient NPDES monitoring station or to bracket known areas or sources of ecological disturbance. Additional reference sites on nearby streams were used for some tasks, depending on the specific objectives of the task.

\section{Toxicity Testing}

The bimonthly evaluation of the toxicity of the discharges from the K-1407-B pond initiated in October 1986 continued through April 1988. Following closure of the K-1407-B pond in October 1988, testing of the effluents from the K-1407-E/F ponds was initiated and continued bimonthly through June 1990. Similarly, water from the K-1407-J basin was tested for toxicity at bimonthly intervals from 
December 1988 through June 1990. The effluent from storm drain (SD) 170 was evaluated for toxicity eight times, and the toxicity of effluents from SDs 180 and 190 was evaluated six times each between July 1988 and May 1990. The toxicity of the effluents (grab samples) was determined by 7-d static-renewal tests that measured survival and growth of fathead minnow (Pimephales promelas) larvae and survival and reproduction of a microcrustacean (Ceriodaphnia dubla). Toxicity patterns were similar for the $\mathrm{K}-1407-\mathrm{B}$ pond, the K-1407-E/F ponds, and the K-1407-J basin. Effluents from these basins/ponds were either never toxic (K-1407-E/F ponds) or rarely toxic (K-1407-B pond and K-1407-J basin) to fathead minnows but were nearly always toxic to Ceriodaphnia. The toxicity of the effluents from the K-1407-E/F ponds appeared to be linked to constituents that cause high hardness and conductivity levels, whereas the toxicity of effluent from the K-1407-J basin appeared to be related, in part, to elevated concentrations of sodium, chloride, and sulfate. The effluent from SD 170 was always toxic to Ceriodaphnia and frequently toxic to fathead minnows. Similarly, the effluent. from SD 190 was almost always toxic to Ceriodaphnia and occasionally toxic to fathead minnows, whereas the effluent from SD 180 was occasionally toxic to both species. Dechlorination did not always remove toxic components from the storm drains, suggesting that toxic constituents other than chlorine were sometimes present.

The ambient (instream) toxicity at six sites in Mitchell Branch was evaluated bimonthly from January 1987 through July 1990 by using the same testing protocols that were used to evaluate the toxicity of point-source discharges. Water from the two sites farthest upstream in Mitchell Branch [Mitchell Branch kilometers (MIKs) 1.43 and $1.0 \mid$ adversely affected fathead minnows but not Ceriodaphnia. Because there were no obvious sources of toxicants at either of these sites, a bacterial or fungal fish pathogen may have been involved. Water from MIK 0.71 downstream to MIK 0.45 showed strong evidence of acute toxicity to both species through 1988. In 1989 and 1990, the incidence of acute toxicity at these sites declined. Water from MIK 0.12 was never acutely toxic to fathead minnows but was acutely toxic to Ceriodaphnia in many tests conducted from 1987 through 1989.

Chronic toxicity to fathead minnows was evident only in water from MIK 0.54 , whereas chronic toxicity was detected in Ceriodaphnia in water from MIKs 0.71, 0.54 , and 0.12 . Evidence to date suggests that chlorine appears to be the primary toxicant contributing to the acute toxicity of water from some sites. Chronic toxicity may be caused by low levels of total residual chlorine from the storm drains, constituents released from the K-1407-E/F ponds, and/or unidentified area-source contamination.

\section{Bioaccumulation Studies}

Results of monitoring polychlorinated biphenyl (PCB) accumulation in aquatic biota in Mitchell Branch and downstream aquatic systems from 1987 through 1990) continued to show that Mitchell Branch was clearly a source of PCB contamination to its biota during this period. PCB monitoring also suggested that Mitchell Branch may be a significant source of $\mathrm{PCB}$ contamination to lower Poplar Creek. Some fish in Mitchell Branch contained PCB concentrations in excess of the $2 \mu \mathrm{g} / \mathrm{g}$ U.S. Department of Agriculture Food and Drug Administration (FDA) action limit (FDA 1984a), and accumulation of PCBs in caged Asiatic clams (Corbicula fluminea) was also substantial. Significant PCB contamination was also evident in some 
species of fish collected from Poplar Creek a short distance downstream of Mitchell Branch. However, the significance of Mitchell Branch as a source of PCBs in Poplar Creek and the Clinch River arm of Watts Bar Reservoir cannot yet be determined. Except for slightly elevated concentrations of polycyclic aromatic hydrocarbons in caged clams, no elevated concentrations of other organic compounds were positively identified in caged clams.

Mercury concentrations in Mitchell Branch fish continue to exceed background levels, but the levels were not higher than the FDA limit (FDA 1984b) and were typical of the degree of contamination found in fish in nearby Poplar Creek. Thus, the significance of Mitchell Branch as a source of mercury to its biota, and to the biota of downstream aquatic systems, was inconclusive. Concentrations of other metals were not elevated in fish from Mitchell Branch.

\section{Population and Community Studies}

Studies of fish and benthic invertebrates continued to show that the biota in Mitchell Branch downstream of SD 170 are severely affected by operations at the Oak Ridge K-25 Site. Furthermore, results from benthic invertebrate studies showed evidence of substantial impact at the two monitoring sites located immediately upstream of SD 170 (i.e., MIKs 0.78 and 0.86). Fish were absent from all three sampling sites downstream of SD 170 (i.e., MIKs 0.71, 0.54, and 0.45), and they tended to congregate at MIK 0.78 , just upstream of SD 170. The benthic invertebrate community was strongly stressed at MIK 0.71, whereas minor improvements were evident further downstream. Results from ambient toxicity tests, analyses of the structure and composition of the benthic macroinvertebrate community, and the absence of fish in lower Mitchell Branch suggest that the biota near SDs 170, 180, and 190 were influenced primarily by frequent releases of one or more toxicants such as chlorine. The lack of toxicity to Ceriodaphnia in ambient tests of water from MIK 1.10, plus the congregation of fish upstream of all major discharges, suggests that the impact observed in the benthic invertebrate community at MIKs 0.78 and 0.86 may be the result of a nontoxic stress (e.g., siltation from construction activities).

The health of redbreast sunfish (Lepomis auritus) in lower Mitchell Branch was assessed by observing a suite of biochemical and physiological parameters. These parameters provided information in five functional categories: (1) detoxification enzyme induction, (2) organ dysfunction, (3) histopathology, (4) overall fish health and condition, and (5) nutritional status. Results from this study indicated that redbreast sunfish appeared to be physiologically stressed. Evidence suggesting exp sure to toxicants and/or poor water quality included (1) elevation of a detoxification enzyme, (2) gill dysfunction, and (3) evidence of some liver impairment. There was also evidence of indirect stress related to nutritional status; fish in Mitchell Branch generally had less food in their stomachs and intestines, and the level of serum triglycerides was low relative to fish from reference sites.

\section{Future Studies}

Results of studies conducted since 1986 in association with the Oak Ridge K-25 Site BMAP will be used to guide future monitoring efforts. Sampling sites and frequencies will remain the same for the effluent and ambient toxicity studies and benthic macroinvertebrate and fish studies, although the frequency of benthic invertebrate sample collection was reduced to quarterly after the second year of sampling (i.e., quarterly beginning August 1988). Bioaccumulation studies will key on 
identifying the source(s) of PCBs and the significance of Mitchell Branch as a source of mercury to its biota. Bioindicator studies will continue largely as before but will also include the evaluation of reproductive competence of the target species. Although routine monitoring of Mitchell Branch will continue, increasing emphasis will be placed on development and testing of hypotheses on the factors and mechanisms contributing to the adverse conditions that have been observed to date. Ultimately, the rate of recovery of the biotic communities, the elimination of toxicity in the middle reaches of Mitchell Branch, and the reduction of contaminant residues in biota of Mitchell Branch will all depend on accurate identification of the causal factor(s). 


\title{
1. INTRODUCTION
}

\author{
J. G. Smith
}

On September 11, 1986, a modified National Pollutant Discharge Elimination System (NPDES) permit was issued for the Oak Ridge Gaseous Diffusion Plant (ORGDP; now referred to as the Oak Ridge K-25 Site), a former enricheduranium production facility currently operated by Martin Marietta Energy Systems, Inc., for the U.S. Department of Energy (DOE). As specified in Part III (L) of the permit, a plan for the biological monitoring of the receiving stream (K-1700 stream or Mitchell Branch) was submitted for approval to the U.S. Environmental Protection Agency (EPA) and the Tennessee Department of Environment and Conservation (TDEC) in December 1986. Because it was anticipated that the chemical composition of several effluents would be altered shortly after the permit modifications were issued, the Oak Ridge K-25 Site Biological Monitoring and Abatement Program (BMAP) was implemented in August 1986, before the plan had been formally approved by the regulatory agencies.

The BMAP for Mitchell Branch was developed to meet two major objectives. First, studies were designed to provide sufficient data to determine whether the interim effluent limits established for the K-25 Site protect and maintain the use of Mitchell Branch for growth and propagation of fish and other aquatic life. A second major objective was to document the effects on stream biota resulting from construction and operation of major new pollution abatement facilities, including the Central Neutralization Facility (CNF) and the Toxic Substances Control Act (TSCA) incinerator. The ecological effects of remedial actions (e.g., closure of the $\mathrm{K}-1407-\mathrm{B}$ and $\mathrm{K}-1407-\mathrm{C}$ holding ponds) can also be evaluated by this monitoring program.

The effluents discharged to Mitchell Branch are chemically complex and contain various trace metals, organic chemicals, neutral salts, and radionuclides (Sect. 2.2). Moreover, the composition of these effluents will change as various pollution abatement measures are implemented over the next several years. Although contaminant inputs to the stream originate primarily as point-sources from existing plant operations, area or non-point sources such as the classified burial grounds cannot be eliminated as potential sources of contaminants. A multitiered, integrated approach to biological monitoring was developed to address this complexity. The Mitchell Branch BMAP consists of four major tasks: (1) effluent and ambient toxicity testing, (2) bioaccumulation studies, (3) biological indicator studies, and (4) ecological surveys of stream communities (e.g., benthic macroinvertebrates and fish). Because few fish occur in Mitchell Branch, the third task, biological indicator studies, will use hatchery-reared species as needed. These in troduced fish will be maintained in enclosures in the stream. 


\title{
2. DESCRIPTION OF STUDY AREA
}

\author{
R. L. Hinzman and J. M. Loar
}

Mitchell Branch is a small stream located near the northeast boundary of the Oak Ridge K-25 Site (Figs. 2.1 and 2.2). The stream has a drainage area of $1.78 \mathrm{~km}^{2}$ and is similar in size to upper Grassy Creek [2.59 km² at Grassy Creek kilometer (GCK) 2.4], a reference stream located about $2 \mathrm{~km}$ southeast of the
Oak Ridge K-25 Site (Fig. 2.3). Mitchell Branch flows only $1.5 \mathrm{~km}$ from its headwaters to its mouth, where it drains into Poplar Creek about $150 \mathrm{~m}$ downstream of the Blair Road bridge. The confluence of the two streams is about $1.5 \mathrm{~km}$ below the mouth of East Fork Poplar Creek (EFPC) and $7 \mathrm{~km}$ above

ORNL-DWG 94M-4972

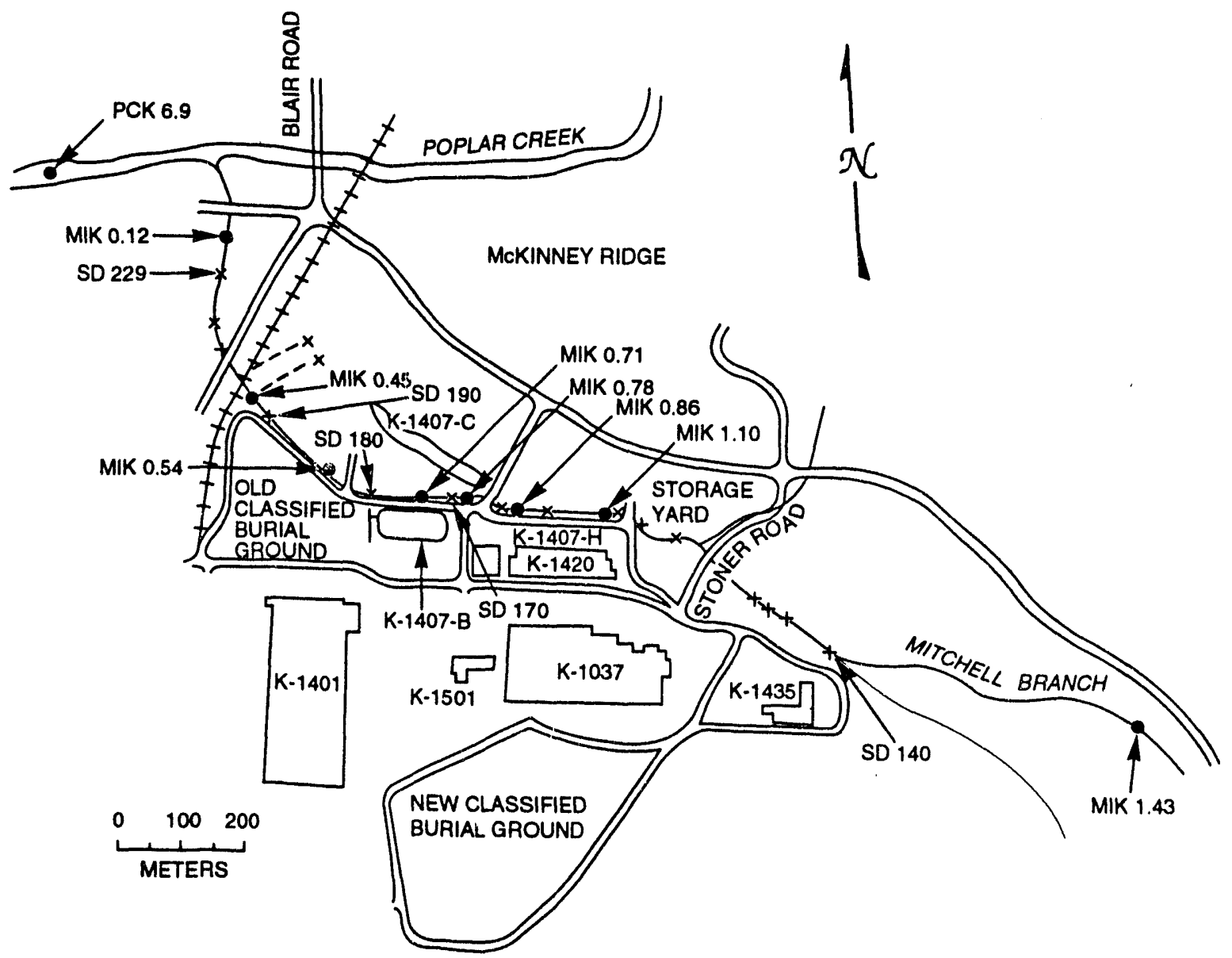

Fig. 2.1. Map of Mitchell Branch and a portion of the Oak Ridge K-25 Site showing the locations of the biological monitoring sites $(\bullet)$ in relation to the storm drains $(x)$ and the K-1407-B pond, which closed after October 31, 1988. 


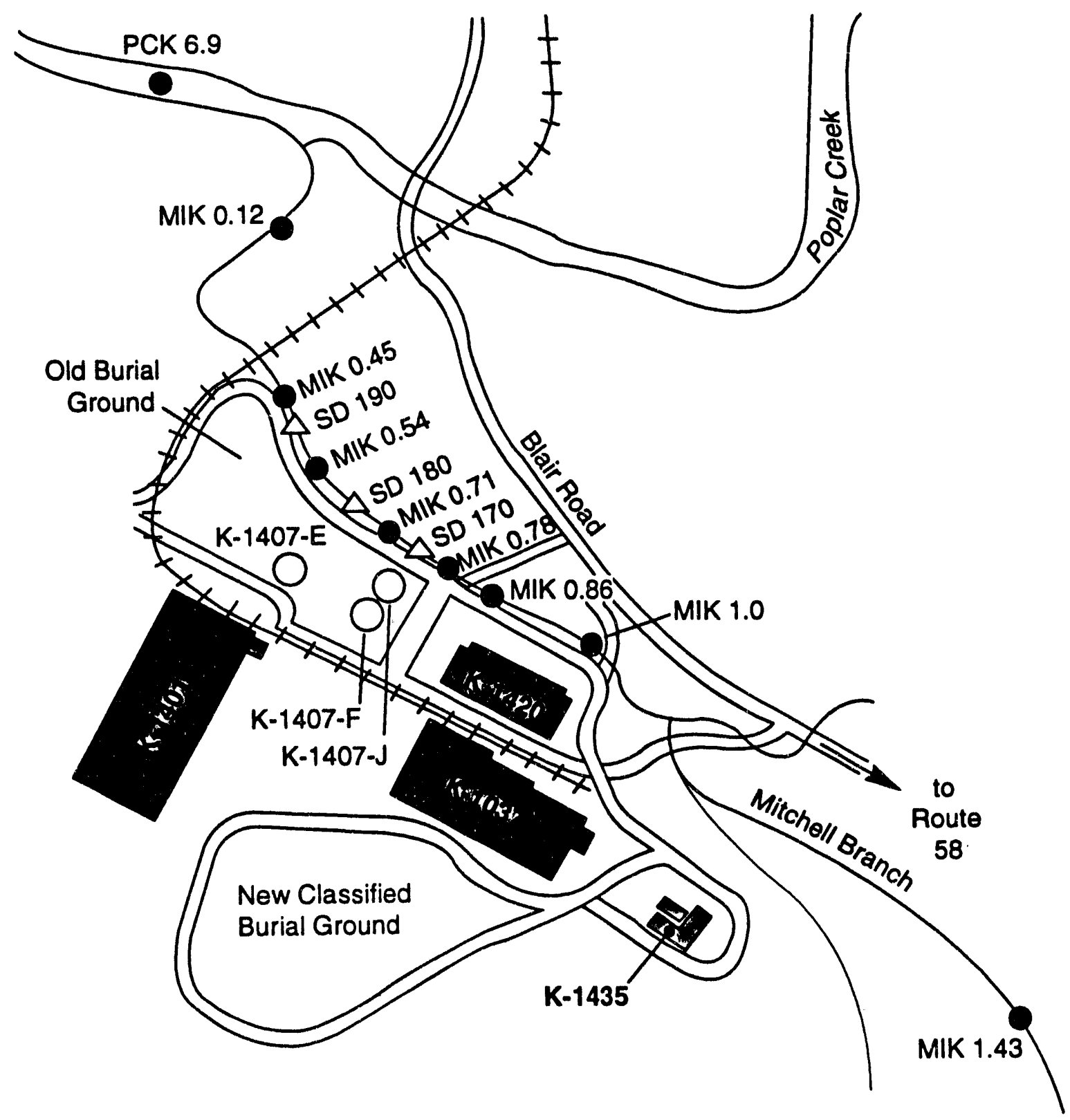

Fig. 2.2. Map of Mitchell Branch and a portion of the Oak Ridge K-25 Site showing the locations of biological monitoring sites $(\bullet)$ in relation to selected storm drains $(\Delta)$ and the $K-1407-E / F$ ponds and the K-1407 $J$ basin, which replaced the K-1407-B pond on November 1, 1988.

the confluence of Poplar Creek with the Clinch River (Fig. 2.3).

Poplar Creek, which has a drainage area of $352 \mathrm{~km}^{2}$, originates northeast of Oliver Springs, Tennessee, on the
Cumberland Plateau (Figs. 2.1 and 2.3). The general direction of streamflow of Poplar Creek is southeasterly, entering the DOE Oak Ridge Reservation (ORR) north of the K-25 Site, and then flowing 


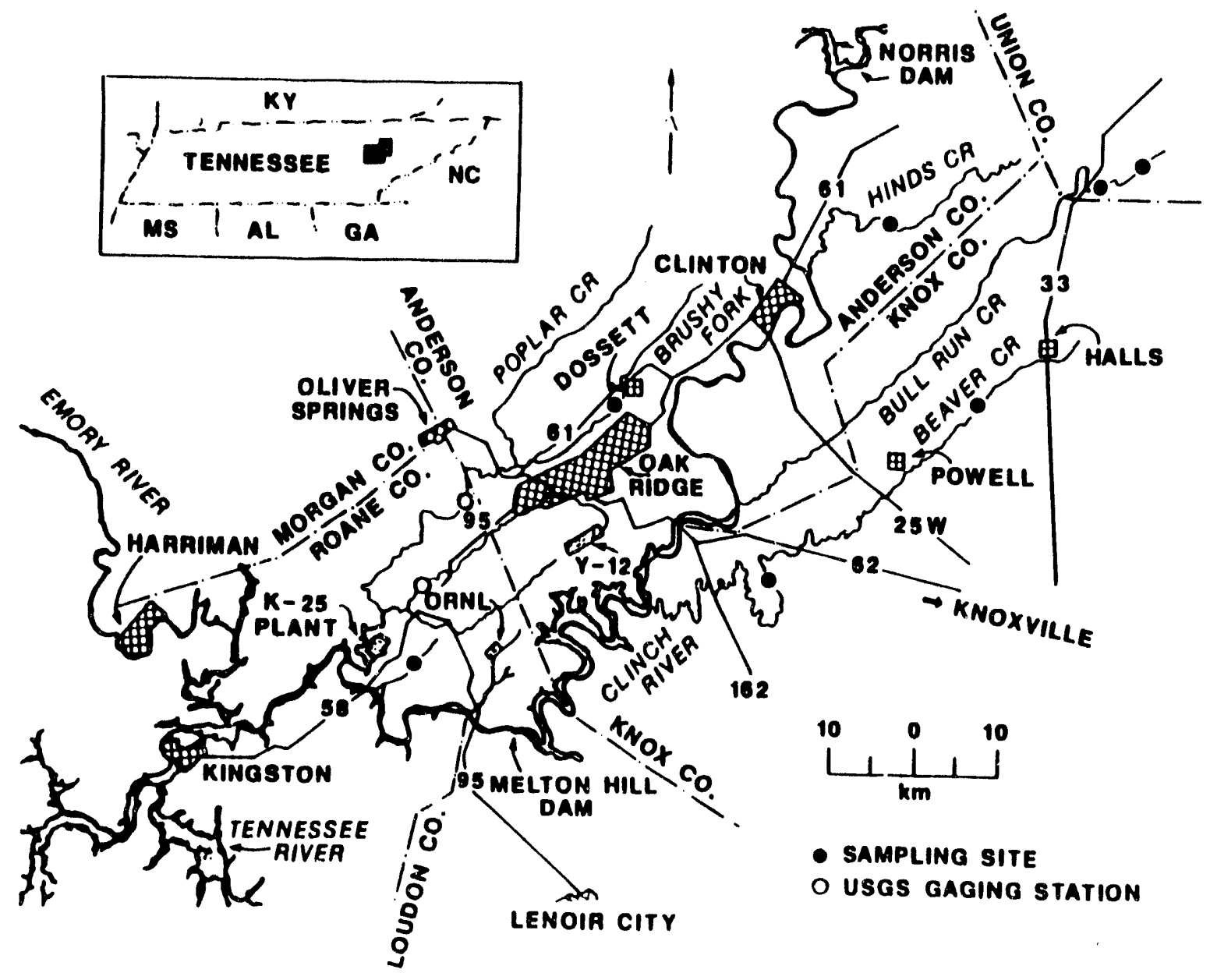

Fig. 2.3. Map of the Oak Ridge area showing locations of the reference (control) sites.

for a distance of $10 \mathrm{~km}$ through the plant area before entering the Clinch River near Clinch River kilometer (CRK) 19.3. EFPC, a major tributary of Poplar Creek, originates from springs on the northwest slope of Chestnut Ridge within the boundaries of the Oak Ridge Y-12 Plant. Streamflow is controlled by Lake Reality, a 1.0-ha settling pond located $\sim 1.5 \mathrm{~km}$ below the spring. The creek flows for $\sim 24 \mathrm{~km}$ before joining the West Fork at Poplar Creek kilometer (PCK) 8.8.

\subsection{GEOHYDROLOGY}

\subsubsection{Mitchell Branch}

Mitchell Branch originates near the base of a small knoll southwest of McKinney Ridge. The knoll is underlain by the Conasauga Group, which consists of a calcareous shale interbedded with thinner layers of limestone and siltstone (DOE 1979). Streams such as Mitchell Branch, which are underlain by shale and sandstone, have a smaller low-flow discharge 
and greater range in flow than streams underlain by carbonate rocks such as Knox Dolomite (McMaster 1967).

Periods of zero discharge sometimes occur in portions of Mitchell Branch just upstream of the BMAP sampling site at Mitchell Branch kilometer (MIK) 0.86 [J. G. Smith, Environmental Sciences Division (ESD), Oak Ridge National Laboratory (ORNL), personal observation]. Periods of zero discharge are also characteristic of the upper reaches of Melton Branch near ORNL (Loar 1990, Table 2.1), a small stream that is also underlain predominantly by shale and sandstone (McMaster 1967, Table 10).

Discharges from the Oak Ridge K-25 Site augment the flow of Mitchell Branch downstream of MIK 0.78. Once-through cooling water and process water account for about $21 \%$ and $10 \%$, respectively, of the streamflow at NPDES monitoring station K-1700 on lower Mitchell Branch at MIK 0.12 (Kasten 1986). Surface runoff probably accounts for most of the remaining flow (69\%) (Kasten 1986), although there is a minor contribution from groundwater (Scheib 1987, Table 7). Based on these estimates, $-31 \%$ of the flow in lower Mitchell Branch can be attributed to discharges from the Oak Ridge K-25 Site. However, in years of below-normal precipitation and minimal runoff, such as in 1985-87, nearly $100 \%$ of the flow in the stream could be plant effluent. The potential benefit to biota derived from increasing the minimum flow in the stream (i.e., reduction in stream bed dewatering and habitat loss) could be offset by the adverse impacts of insufficient dilution of these effluents.

Mitchell Branch has a relatively low temporal variability in discharge volume compared with streams without flow augmentation (Table 2.1). The variability in discharge in Mitchell Branch was higher than in EFPC at East Fork Poplar Creek kilometer (EFK) 5.3, where flow is augmented by $-50 \%$ in dry years. The effect of flow augmentation may be beneficial to some biota. For example, Horowitz (1978) found that greater numbers of fish species occurred in streams where flow was more constant.

The mean annual flow (MAF) in Mitchell Branch decreased by $\sim 25 \%$ in 1986 and again in 1987 (Table 2.1). Placing the Oak Ridge K-25 Site on standby status during this period reduced the releases of water from small, oncethrough cooling systems by almost $50 \%$ (Kasten 1986, Fig. 12). Because water from these systems accounts for less than $25 \%$ of the flow in Mitchell Branch, they probably contributed less to the reduction in stream discharge than the below-normal precipitation that occurred from 1985-88. Generally, streams with and without flow augmentation decreased in flow from 1985-87 (Table 2.1). Even in 1985, which was used as a baseline for this comparison, the MAFs in Poplar Creek and EFPC were only $62 \%$ and $79 \%$, respectively, of the historical MAF (period of record: 1960-85) (Lowery et al. 1987).

Although precipitation was below normal from 1985-88, the Mitchell Branch hydrograph in 1985 differed substantially from those for 1986-89 (Appendix A, Fig. A.1). Flows were generally less variable in 1985 (Table 2.1), and there was no prolonged low-flow period, which was a dominant feature of the 1986-89 hydrographs. During a 5-month period from summer through early fall, mean monthly flows were below 450 and $350 \mathrm{~L} / \mathrm{min}$ in 1986 and 1987, respectively; for two consecutive months in both years, flows averaged less than $150 \mathrm{~L} / \mathrm{min}$. In comparison, mean monthly flows in 1985 never fell below $500 \mathrm{~L} / \mathrm{min}$ and were never less than $700 \mathrm{~L} / \mathrm{min}$ for more than 2 consecutive months. Also, the minimum daily flow in 1985 was almost an order of magnitude higher than the minimum flows in 1986 and 1987. Annual precipitation was 
Table 2.1. Comparison of mean annual discharge (in liters per second) and percentage change between years for local streams with and without significant flow augmentation

\begin{tabular}{|c|c|c|c|c|}
\hline \multirow[b]{2}{*}{ Year } & \multicolumn{2}{|c|}{ With flow augmentation } & \multicolumn{2}{|c|}{ Without flow augmentation } \\
\hline & $\begin{array}{l}\text { Mitchell } \\
\text { Branch }\end{array}$ & $\begin{array}{l}\text { East Fork Poplar } \\
\text { Creek (EFK 5.3) }\end{array}$ & $\begin{array}{c}\text { Upper Melton } \\
\text { Branch (MEK 1.93) }\end{array}$ & $\begin{array}{l}\text { Bear Creek } \\
\text { (BCK 4.55) }\end{array}$ \\
\hline 1985 & $16.7(50.0)$ & $1114(33.8)$ & a & $a$ \\
\hline 1986 & $12.5(81.6)$ & $1011(52.1)$ & $10.5(137.1)$ & $107(110.6)$ \\
\hline 1987 & $9.6(70.8)$ & $998(50.6)$ & $10.2(148.4)$ & $111(110.6)$ \\
\hline 1988 & $23.1(48.8)$ & $1100(147.7)^{b}$ & $9.1(469.3)$ & $92(238.9)$ \\
\hline 1989 & $23.7(25.5)$ & NA & $28.0(187.1)$ & $325(145.8)$ \\
\hline \multicolumn{5}{|c|}{ Percentage change } \\
\hline $1985-1986$ & -25.2 & -9.2 & NA & NA \\
\hline 1986-1987 & -23.2 & -1.3 & -2.9 & 3.7 \\
\hline $1987-1988$ & 140.6 & 9.2 & -10.8 & -17.2 \\
\hline $1988-1989$ & 2.6 & NA & 207.7 & 253.3 \\
\hline
\end{tabular}

${ }^{\triangle}$ New USGS station; no record prior to April 1, 1985, for Melton Branch or before March 1, 1985 for Bear Creek.

'Values based on $182 \mathrm{~d}$ of record; station was discontinued after June 30, 1988.

Note: Values in parentheses are the coefficient of variation $(\mathrm{CV})$ for the mean flow values. The mean and $\mathrm{CV}$ were computed from mean monthly values taken from monthly NPDES reports (Mitchell Branch at MIK 0.12). EFK = East Fork Poplar Creek kilometer; MIK = Mitchell Branch kilometer; $\mathrm{BCK}=$ Bear Creek kilometer.

Sources: Lowery, J. F., et al., 1986, Water Resources Data for Tennessee, Water Year 1985, Report No. USGS/WRD/HD-86/216, U.S. Geological Survey, Nashville, Tennessee; Lowery, J. F., et al., 1987, Water Resources Data for Tennessee, Water Year 1986, Report No. USGS/WRD/HD-87/225, U.S. Geological Survey, Nashville, Tennessee; Lowery, J. F., et al., 1988, Water Resources Data for Tennessee, Water Year 1987, Report No. USGS/WRD-HD-88/236, U.S. Geological Survey, Nashville, Tennessee; Lowery, J. F., et al., 1989, Water Resources Data for Tennessee, Water Year 1988, Report No. USGS/WRD-HD-89/258, U.S. Geological Survey, Nashville, Tennessee, and USGS provisional discharge data [L. D. Voorhees, ORNL, Environmental Sciences Division (ESD), unpublished data].

$124.3 \mathrm{~cm}$ in 1988 and $167.7 \mathrm{~cm}$ in 1989 (90\% and $120 \%$ of normal, respectively, for the 1951-80 period of record), which increased mean monthly flows in both years; during this same period, flows fell below $500 \mathrm{~L} / \mathrm{min}$ only once (September 1989).
The number of days of zero discharge in upper Melton Branch also provides a relative measure of ecologically meaningful differences in the hydrographs of the past 3 years. For example, no flow was recorded for $104 \mathrm{~d}$ in 1986, $172 \mathrm{~d}$ in 1987, and 141 days in 1988 (Table 2.2). More 
Table 2.2. Number of days of zero discharge (number of consecutive days in parentheses) in upper Melton Branch at USGS gaging station 03537100 ncar Oak Ridge National Laboratory

\begin{tabular}{llllll}
\hline & 1985 & 1986 & 1987 & 1988 & 1989 \\
\hline May & 0 & 0 & 0 & $2(2)$ & 0 \\
June & $4(2)$ & $10(10)$ & $11(8)$ & $30(30)$ & 0 \\
July & $8(6)$ & $15(15)$ & $21(19)$ & $21(12)$ & $1(1)$ \\
August & 0 & $31(31)$ & $31(31)$ & $30(26)$ & $21(17)$ \\
September & $2(2)$ & $27(24)$ & $30(30)$ & $25(11)$ & $7(5)$ \\
October & 0 & $20(12)$ & $31(31)$ & $31(31)$ & 0 \\
November & 0 & 0 & $30(30)$ & $3(3)$ & 0 \\
December & 0 & 0 & $\frac{18(14)}{172(155)}$ & $\frac{0}{142(45)}$ & $\frac{1}{29(17)}$ \\
Total & $14(6)$ & $103(47)$ & & & \\
\hline
\end{tabular}

Sources: Lowery, J. F., et al., 1986, Water Resources Data for Tennessee, Water Year 1985, Report No. USGS/WRD/HD-86/216, U.S. Geological Survey, Nashville, Tennessee; Lowery, J. F., et al., 1987, Water Resources Data for Tennessee, Water Year 1986, Report No. USGS/WRD/HD-87/225, U.S. Geological Survey, Nashville, Tennessee; Lowery, J. F., et al., 1988, Water Resources Data for Tennessee, Water Year 1987, Report No. USGS/WRD-HD-88/236, U.S. Geological Survey, Nashville, Tennessee; Lowery, J. F., et al., 1989, Water Resources Data for Tennessee, Water Year 1988, Report No. USGS/WRD-HD-89/258, U.S. Geological Survey, Nashville, Tennessee; Lowrey, J. F., et al., 1990, Water Resources Data for Tennessee, Water Year 1989, Report No. USGS/WRD/TN-89-1, U.S. Geological Survey, Nashville, Tennessee, and USGS provisional discharge data [L. D. Voorhees, ORNL, Environmental Sciences Division (ESD), unpublished data].

important, the number of consecutive days with zero discharge in 1987 (155 d) exceeded the number in 1986 and 1985 by factors of 3 and 25 , respectively, and in $1988(113 \mathrm{~d})$ by factors of 2 and 18 respectively. These data indicate that any adverse ecological effects resulting from reduced streamflow were also greater in 1987 because of below-normal precipitation that year and the previous year. Thus, the first year of biological monitoring was conducted over extreme, and possibly worst case, ambient conditions.

\subsubsection{Poplar Creek}

Poplar Creek is the largest tributary of the Clinch River between Melton Hill Dam and the northwest boundary of ORR. It has an average annual discharge that is approximately ten times greater than the combined discharges of other tributaries in this $21-\mathrm{km}$ reach of the river (Loar et al. 1981). It flows from Poplar Creek Valley, which is underlain by the Conasauga shale, through a gap in Black Oak Ridge, which is underlain by the Knox Formation, and enters the Clinch River southeast of the 
Oak Ridge K-25 Site. With the exception of the silty shale and siltstone-sandstone members of the Rome Foundation adjacent to Route 58 , southeast of the plant, most of the K-25 Site is underlain by Chickamauga Limestone (DOE 1979).

Poplar Creek exhibits seasonal fluctuations in discharge that, in general, reflect precipitation and runoff patterns typical of this region of East Tennessee. Maximum precipitation is in the winter (December-February), when $\sim 31 \%$ of the annual precipitation occurs, the wettest months being February and March (NOAA 1990). Maximum runoff is also likely to occur in January, February, or March, when rain- fall is normally high and soil moisture and groundwater storage are highest (McMaster 1967). As shown in Fig. 2.4, the discharge in Poplar Creek was highest during the winter months and lowest in late summer, when rainfall is normally low and runoff is minimal. Water levels in Poplar Creek are also influenced by the operation of two Tennessee Valley Authority (TVA) dams: Melton Hill Dam

ORNL-DWG 94-6038

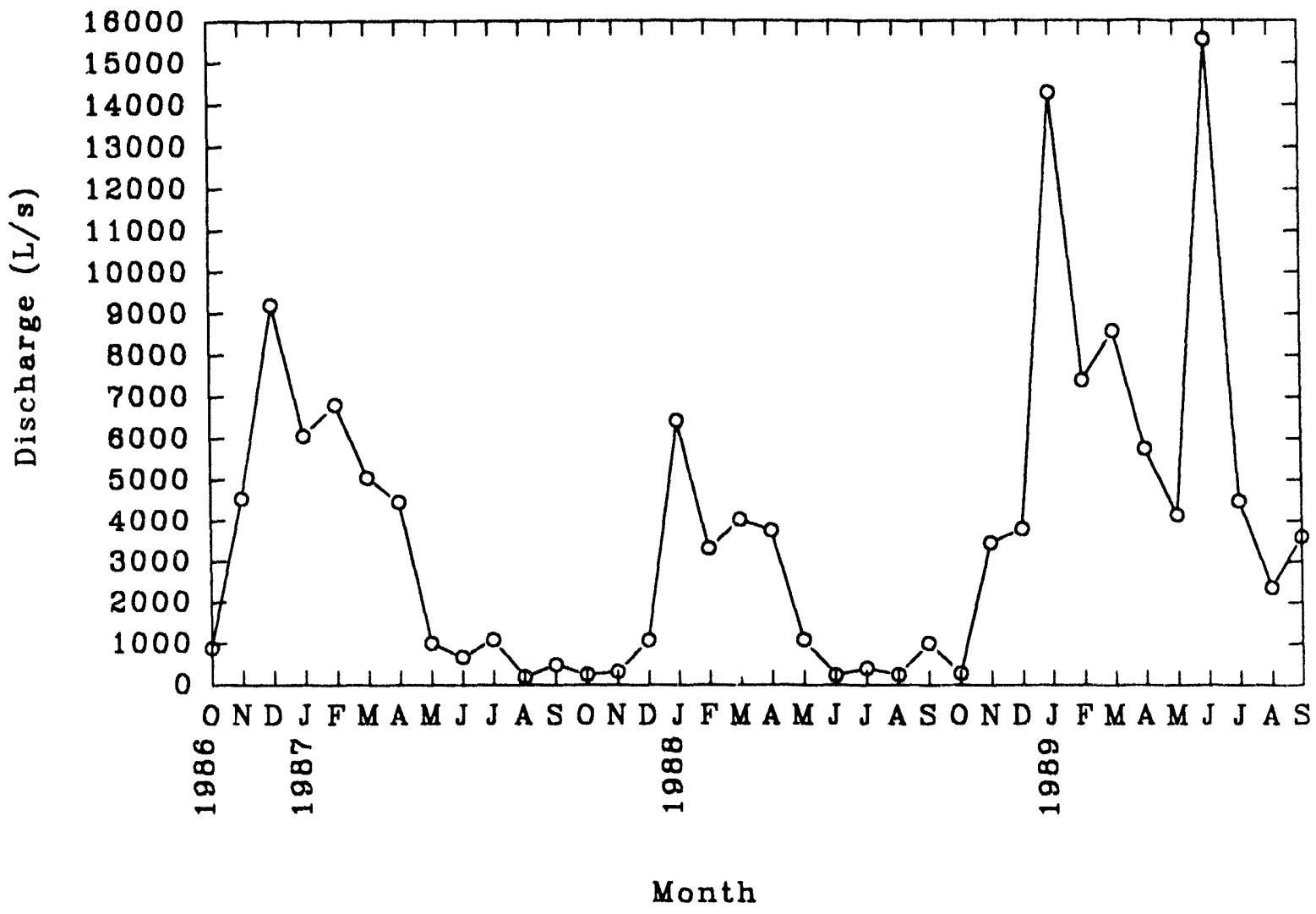

Fig. 2.4. Mean monthly discharge in Poplar Creek. The value for June 1989 is estimated. Source: J. F. Lowery et al., 1987, Water Resources Data for Tennessee Water Year 1986. Repont No. USGSMRD/HD-87/225, U.S. Geological Survey, Nashville, Tenn.; J. F. Lowery et al., 1988, Water Resources Data for Tennessee Water Year 1987. Report No. USGSMRD/HD-88/236, U.S. Geological Survey, Nashville, Tenn.; J. F. Lowery et al., 1989, Water Resources Data for Tennessee Water Year 1988. Report No. USGS/WRD/HD-89/258, U.S. Geological Survey, Nashville, Tenn. 
at CRK 37.2 (completed in 1963) and Watts Bar Dam located at Tennessee River kilometer (TRK) 852 (completed in 1942). Dam operations not only affect the magnitude and frequency of water level fluctuations, but they also influence stream velocities and flow direction in the lower reaches of Poplar Creek.

\section{WATER QUALITY}

Water quality of Mitchell Branch is influenced not only by the geology of the drainage basin (Sect. 2.1) but also by (1) effluents that enter the stream via the $\mathrm{K}-1407-\mathrm{E} / \mathrm{F}$ ponds and storm drains, (2) lesachate from waste disposal sites (i.e., area-source discharges), and (3) remedial action projects. The following characterizations of water quality are preceded by general descriptions of the sources of effluents discharged by the Oak Ridge K-25 Site. The characterizations of water quality are based on data from NPDES monitoring station K-1700 (Sect. 2.2.2.1), effluent data from the K-1407-J basin (Sect. 2.2.2.2), and BMAP-related measurements of water temperatures (Sect. 2.2.3). Water quality, characterizations of Mitchell Branch associated with toxicity testing are given in Sect. 3.2.3.1.

\subsection{Description of the Oak Ridge K-25 Site Discharges}

\subsubsection{Mitchell Branch}

Point-source discharges to Mitchell Branch from current Oak Ridge K-25 Site operations generally fall into one of two categories: process water or storm drain effluents. Prior to October 1988, wastes from the uranium recovery facility, the metals cleaning facility, the chemical process development facility, the steam plant, and the coal yard were neutralized in a $113,500-\mathrm{L}$ vat prior to discharge into the K-1407-B pond. Metal hydroxides were allowed to settle to the bottom of the pond, and the supernatant was conveyed, via a 1.6-km long ditch, to Mitchell Branch (Fig. 2.1).

Closure of the K-1407-B pond began on October 31, 1988, under the Resource Conservation and Recovery Act. The pond now receives rainwater and surface runoff, which is pumped to the CNF, for treatment before being discharged to Poplar Creek. On November 1, 1988, the K-1407-B pond was replaced with the $\mathrm{K}-1407-\mathrm{E}$ and $\mathrm{K}-1407-\mathrm{F}$ ponds and the $\mathrm{K}-1407-\mathrm{J}$ settling basin (Figs. 2.2 and 2.5). Prior to September 1, 1989, the K-1407-E/F ponds and the K-1407-J basin discharged directly into Mitchell Branch through storm drains 170 and 180 (SDs 170 and 180). Currently, effluent from the K-1407-J basin is transferred via pipeline to the 801 area, where it is mixed with Clinch River water, run through a series of baffles, and discharged into Poplar Creek.

Treated effluent from the CNF is pumped to the K-1407-J settling basin. The CNF has two treatment modes, "hazardous" and "nonhazardous". Included in the hazardous treatment mode are effluents from the TSCA incinerator. After treatment, hazardous waste is pumped into the K-1407-J basin and then discharged. Nonhazardous treated effluents, such as coal pile runoff and steam plant effluent, are treated in the CNF and then discharged into the K-1407-E/F ponds (Fig. 2.5).

Most of the process water currently being discharged to Mitchell Branch is via the K-1407-E/F ponds, two holding ponds constructed primarily for settling solids and $\mathrm{pH}$ control (K-1407-E, volume $\approx 781,000$ $\mathrm{L} ; \mathrm{K}-1407-\mathrm{F}$, volume $\approx 132,000 \mathrm{~L}$ ). The K-1407-E/F ponds receive primarily caustic wastes from the steam plant water treatment process and coal yard runoff. The 
ORNL.DWG $91 \mathrm{M}-15139$

FINAL SYSTEM

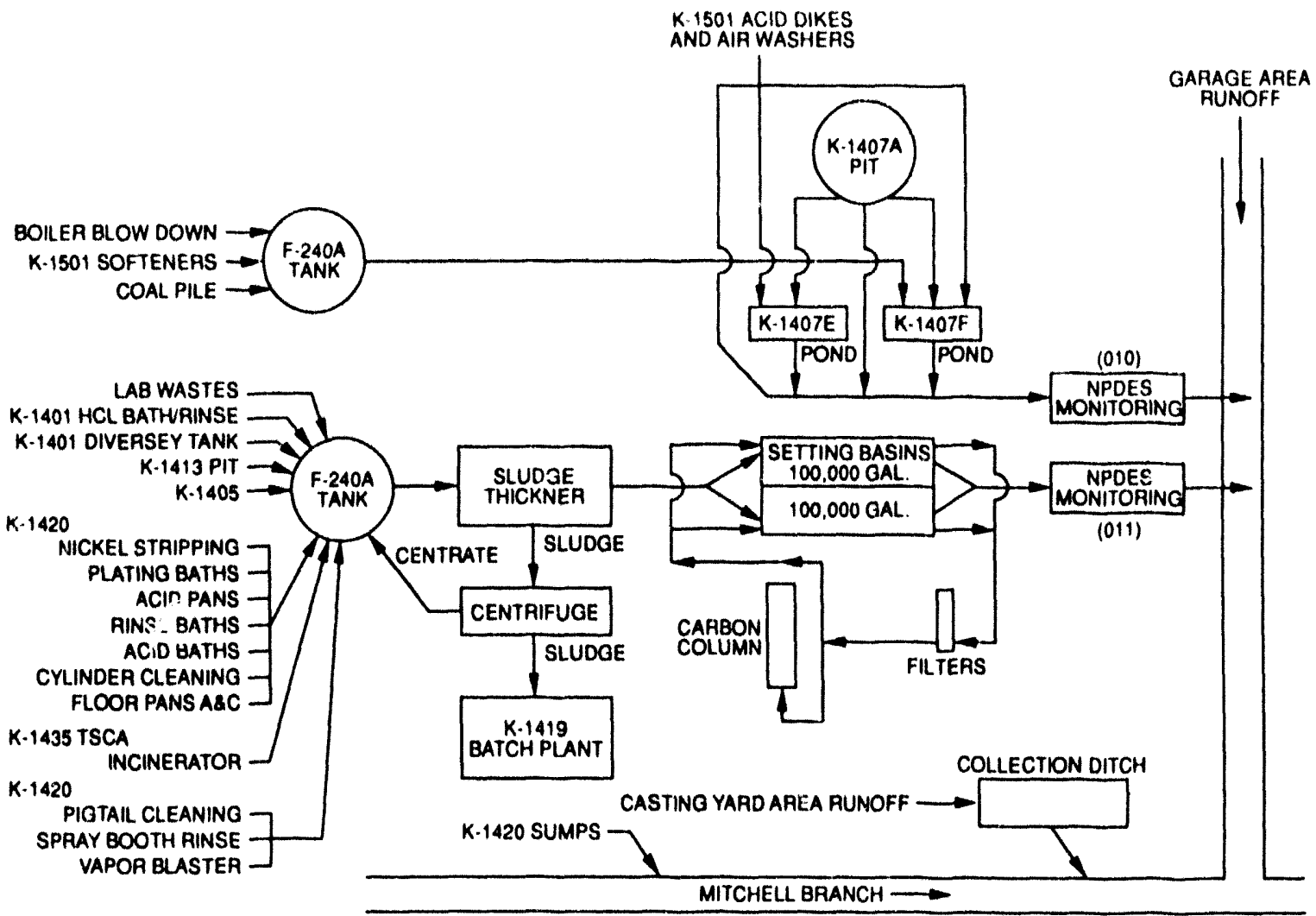

Fig. 2.5. Diagram of effluent input into the Central Neutralization Facility and ponds K-1407-E/F. Source: National Pollutant Discharge Elimination System permit for Central Neutralization Facility.

overflow from the K-1407-F and K-1407-E ponds discharges to SDs 170 and 180 respectively. Before August 1987, some coal yard runoff and boiler blowdown was discharged directly to the stream through SD 170, just upstream of the K-1407-B pond outfall.

Eighteen storm drains enter Mitchell Branch (Fig. 2.1). Although some of these drains contribute only runoff water and suspended particulate matter to the stream during rainfall events, others may convey discharge groundwater, once-through cooling water, or floor drain wastewater in addition to runoff from roofs and parking lots. In the NPDES permit, storm drains are classified according to their source and potential for contamination. Nine storm drains at the Oak Ridge K-25 Site are classified as category III outfalls (those that may receive untreated process wastewaters), and seven of these nine drains discharge to Mitchell Branch (Smith et al. 1992a).

Leachate from waste disposal sites (i.e., area-source discharges) may also enter the stream. The old classified burial ground, a 1.50-ha site located $120 \mathrm{~m}$ west of the K-1407-B pond, was created by filling in a large swampy area that drained into Mitchell Branch (Fig. 2.1). This disposal site contains both radioactive and 
nonradioactive wastes. An ephemeral stream drains the site, which is located within the Mitchell Branch watershed. The $\mathrm{K}-1407 . \mathrm{C}$ retention basin has an area of 0.80 ha and is located $120 \mathrm{~m}$ northeast of the K-1407-B pond. The basin was constructed in 1973 and received dredged material from other holding ponds, including K-1407-B. Although the basin has no surface effluent, a groundwater plume extending from the pond toward the stream has been detected (Ashwood et al. 1986).

\subsubsection{Poplar Creek}

The CNF (Building K-1407-H) went on-line in October 1987. The facility treats effluents from (1) Building 1420, a decontamination and recovery facility; (2) Building $14 C 1$, a metals preparation facility and machine shop; and (3) the TSCA incinerator (Building K-1435). The TSCA incinerator will be used to dispose of polychlorinated biphenyls (PCBs) and other hazardous wastes. Liquid discharges from the incinerator will include (1) scrubber blowdown and (2) fire water and rainwater. These (wo waste streams are collected in a surge tank, analyzed, and, as appropriate, discharged to CNF; pumped through carbon-bed absorbers; or pumped to the waste feed tanks for burning in the incinerator (EPA 1986).

Hazardous wastes from CNF are pumped to the K-1407-J basin, which consists of two aboveground settling ponds (volume $=378,000 \mathrm{~L}$ each) with an average monthly discharge that is slightly less than $5 \times 10^{5} \mathrm{~L}$. Effluent from the $\mathrm{K}-1407-\mathrm{J}$ basin is treated in a batch mode; piped to the 801 area; mixed with water from the Clinch River, which flows over a series of baffles; and then discharged to Poplar Creek.

\subsubsection{NPDES Monitoring}

\subsubsection{Mitchell Branch}

The following characterization of water quality in Mitchell Branch is based on routine monitoring of parameters at NPDES station $\mathrm{K}-1700$. This site is located on lower Mitchell Branch (MIK 0.12) downstream of all point-source and most area-source discharges (Figs. 2.1 and 2.2).

From 1986 through 1989 water quality in Mitchell Branch at MIK 0.12 was characterized by (1) moderate levels of dissolved solids and occasionally high levels of turbidity, (2) relatively low levels of nutrients, (3) elevated levels of most metals and some organics, and (4) high temperatures (discussed in Sect. 2.2.3).

Total dissolved solids (TDS) averaged $340 \mathrm{mg} / \mathrm{L}$ from January through July 1985 and $553 \mathrm{mg} / \mathrm{L}$ for the rest of the year (W. J. Scheib, unpublished data from 1985 NPDES monthly reports). Average annual TDS levels also exceeded $500 \mathrm{mg} / \mathrm{L}$ in 1986 and 1987 (Appendix A, Tables A.1-A.5) but were less than $400 \mathrm{mg} / \mathrm{L}$ in 1989. TDS data were not available for 1988 . High levels of TDS were usually associated with high streamflows, although runoff from construction sites adjacent to Mitchell Branch contributed to the periodically high suspended loads in the stream.

Concentrations of nitrogen in Mitchell Branch are low, whereas concentrations of phosphorus are high. Even though concentrations of nitrate nitrogen were higher than background ( $\sim 0.1 \mathrm{mg} / \mathrm{L}$; Boyle et al. 1982, Table 3.16), they averaged less than $1 \mathrm{mg} / \mathrm{L}$ and never exceeded $8 \mathrm{mg} / \mathrm{L}$ at MIK 0.12 (Appendix A, Tables A.1-A.5). Phosphorus is not monitored in Mitchell Branch, but water from the K-1407-J basin had a median phosphorus concentration of $0.405 \mathrm{mg} / \mathrm{L}$, with a maximum recorded value of $9.3 \mathrm{mg} / \mathrm{L}$ in 1989 (Appendix $\mathrm{A}$, 
Table A.6). K-1407-B and K-1407-E/F ponds had median phosphorus concentrations of $<0.2 \mathrm{mg} / \mathrm{L}$, but the K-1407-B pond had a maximum concentration of $8.4 \mathrm{mg} / \mathrm{L}$ in 1988 (Appendix A, Tables A.3 and A.S).

Many of the metals (Appendix A, Tables A.1-A.5) in Mitchell Branch exceeded concentrations that are typical of small, relatively undisturbed streams on the ORR (Boyle et al. 1982, Tables 3.16 and 4.16). Except for aluminum, iron, and zinc, all measured metals had a median concentration that was at or below the detection limit (i.e., via inductively coupled plasma optical emission spectrophotometry) in each of the past 4 years. Of the organics measured in Mitchell Branch, all were above detection limits at least some of the time. Except for the increase in TDS that occurred in July 1985, the water quality of Mitchell Branch has remained relatively constant over the last 4 years (1986-89).

\subsection{Discharges to Poplar Creek}

Effluent from the K-1407-J basin was characterized by high concentrations of TDS in 1988 and 1989, with median values of 2619 and $1358 \mathrm{mg} / \mathrm{L}$, respectively

(Appendix A. Table A.6).

Concentrations of nitrate, phosphorous, and sulfate were all high in K-1407-J, especially during 1989 . Median values for nitrate exceeded background, and the maximum value in 1989 was $1390 \mathrm{mg} / \mathrm{L}$. The median concentration of phosphorous in 1989 was $0.4 \mathrm{mg} / \mathrm{L}$, and the maximum concentration was $9.3 \mathrm{mg} / \mathrm{L}$. The median concentration of sulfate was higher than $600 \mathrm{mg} / \mathrm{L}$ in 1988 and $400 \mathrm{mg} / \mathrm{L}$ in 1989 ; maximum values in these 2 years were 1200 and $1500 \mathrm{mg} / \mathrm{L}$ respectively.

Concentrations of all metals that were monitored from 1988-89 were elevated at some time during this period, and most had median values above the detection limits (Appendix A, Table A.6).

Although most of the organics that were monitored had median values at or below detection limits, most were detectable at some time during the sampling period (Appendix A, Table A.7).

\section{2 .3 Temperature}

Temperature monitoring was initiated in Mitchell Branch on April 9, 1987, at MIK 0.50 immediately below the outfall of SD 190. Data were collected with a Ryan Tempmentor digital temperature recorder, and values were obtained every $20 \mathrm{~min}$. Because of periodic equipment problems, the 3-year record was incomplete.

Through the period of record, Mitchell Branch temperatures were high relative to unaffected streams on ORR. Mean weekly temperatures in Mitchell Branch sometimes exceeded $25^{\circ} \mathrm{C}$, but were generally below $20^{\circ} \mathrm{C}$ in Grassy Creek (Fig. 2.6), a nearby drainage with a similar geology (Sect. 2.1; McMaster 1967, Table 10). Water temperature averaged $\sim 4$ to $8^{\circ} \mathrm{C}$ higher in Mitchell Branch than Grassy Creek, although maximum temperatures in the two streams in June 1987 differed by as much as $19^{\circ} \mathrm{C}$ (Table 2.3).

The temperature of Mitchell Branch is influenced strongly by cooling water discharges (via storm drains) and the retention of effluent in open ponds (K-1407-B prior to October 1988 and $\mathrm{K}-1407-\mathrm{E} / \mathrm{F}$ after this period) prior to discharge just $130 \mathrm{~m}$ above the monitoring station at MIK 0.50. Furthermore, except for the headwaters, there is little canopy cover above MIK 0.50 to help moderate water temperatures. 
()RNI-DWG 94-6039

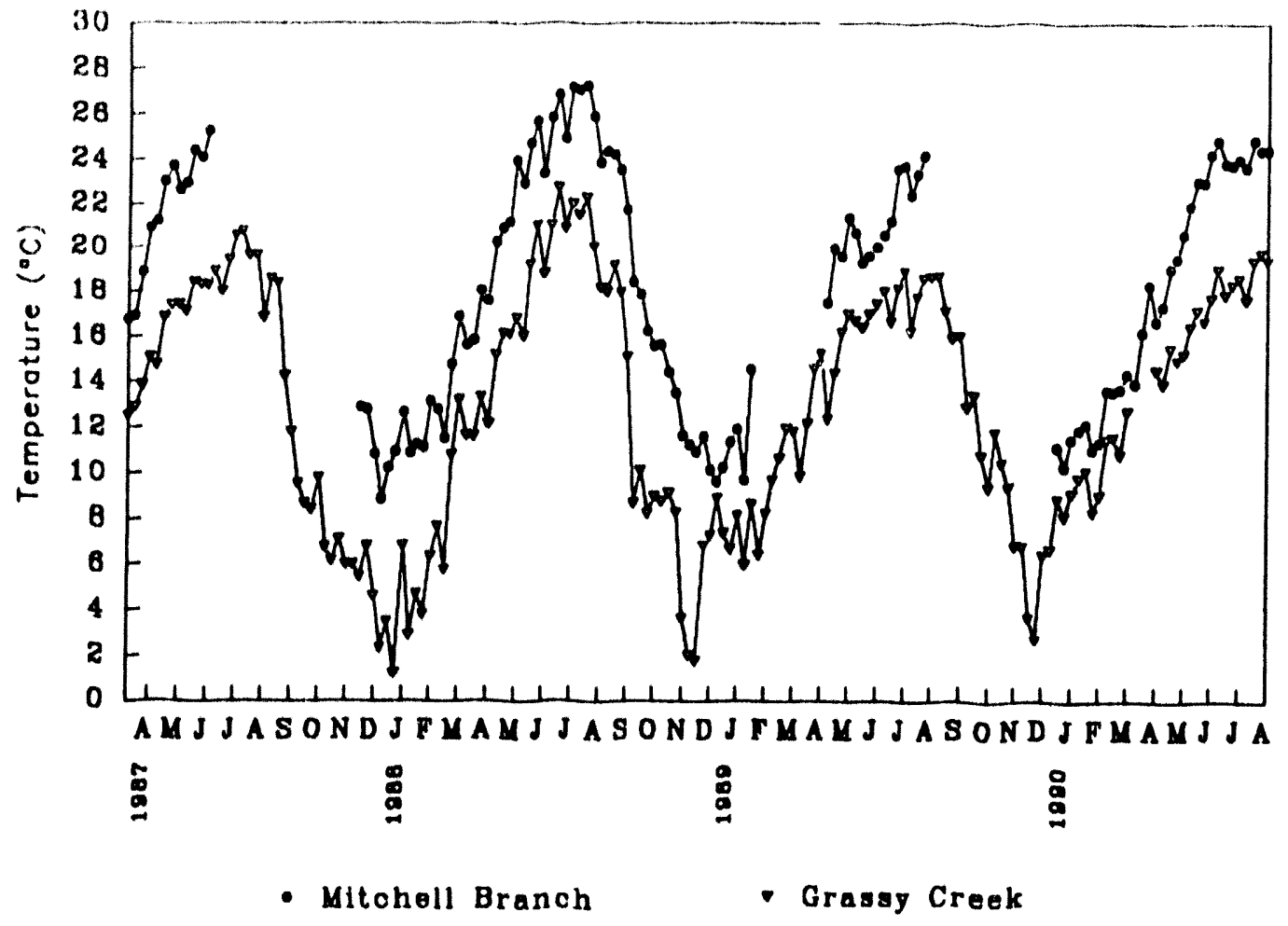

Fig. 2.6. Weekly mean temperatures for Mitchell Branch kilometer 0.50 and Grassy Creek kilometer 2.40 for 1987-90. Temperature record is incomplete as a resuli of equipment failure.

\subsection{BIOLOGICAL MONITORING SITES}

Eight sites on Mitchell Branch

(Figs. 2.1 and 2.2 and Table 2.4) were routinely sampled to assess ecological conditions in the stream. Although the upper site (MIK 1.43) was the primary reference site, sites on other area streams were also used as reference stations

(Fig. 2.3). The lowermost site at MIK 0.12 coincided with the location of the NPDES monitoring station (K-1700). Construction of the weir at MIK 0.12 created a large pool immediately upstream. Mitchell Branch below the weir is also a pool or embayment of Poplar Creek when Watts Bar Reservoir is at full pool (approximately April to October). In this reach of stream, water levels are controlled by operation of Watts Bar Dam, located on the Tennessee River approximately $61 \mathrm{~km}$ downstream of the confluence with the Clinch River. A sampling site for the bioaccumulation task is located at PCK 6.9 downstream of the mouth of Mitchell Branch (Fig. 2.2).

Four of the remaining six sites (MIKs 0.45, 0.54, 0.71, and 0.78) were selected based on the location of the three most significant discharges to Mitchell Branch: SDs 170, 180 (and effluent from the K-1407-E/F holding ponds), and 190 (Figs. 2.1 and 2.2); these monitoring sites are located above and below each of these outfalls. The remaining two sites (MIKs 0.86 and 1.0) were selected to assess the potential for adverse impacts associated with (1) construction activities 
Table 23. Monthly means $( \pm 1 \mathrm{SD}$ ) and range (number of dxys of recond) of water temperature $\left({ }^{\circ} \mathrm{C}\right)$ at Mitchell Branch kilometer (MIK) 0.50 just below the outfall of storm drain 190 and at Grassy Creck kilometer (GCK) 2.4, a reference site

\begin{tabular}{|c|c|c|c|c|c|}
\hline \multicolumn{2}{|c|}{ Sampling period } & \multicolumn{2}{|c|}{ MIK 0.50} & \multicolumn{2}{|c|}{ GCK 2.4} \\
\hline Year & Month & Mean & Range & Mean & Range \\
\hline \multirow[t]{9}{*}{1987} & April & $\begin{array}{l}17.7 \\
(2.1)\end{array}$ & $\begin{array}{c}12.1-24.7 \\
(20)\end{array}$ & $\begin{array}{l}13.2 \\
(2.4)\end{array}$ & $\begin{array}{c}6.8-19.5 \\
(20)\end{array}$ \\
\hline & May & $\begin{array}{l}22.2 \\
(2.2)\end{array}$ & $\begin{array}{c}17.1-29.6 \\
(31)\end{array}$ & $\begin{array}{l}16.0 \\
(2.0)\end{array}$ & $\begin{array}{c}9.9-19.7 \\
(31)\end{array}$ \\
\hline & June & $\begin{array}{l}23.5 \\
(3.9)\end{array}$ & $\begin{array}{c}11.9-41.2 \\
(30)\end{array}$ & $\begin{array}{l}17.8 \\
(1.4)\end{array}$ & $\begin{array}{c}13.3-21.7 \\
(30)\end{array}$ \\
\hline & July & $\begin{array}{l}25.3 \\
(6.4)\end{array}$ & $\begin{array}{c}16.8-41.4 \\
(5.5)\end{array}$ & $\begin{array}{l}18.9 \\
(1.2)\end{array}$ & $\begin{array}{c}15.7-31.1 \\
(28)\end{array}$ \\
\hline & August & $\mathrm{NA}^{a}$ & NA & $\begin{array}{l}20.1 \\
(1.0)\end{array}$ & $\begin{array}{c}16.8-22.0 \\
(31)\end{array}$ \\
\hline & September & NA & NA & $\begin{array}{l}16.9 \\
(2.0)\end{array}$ & $\begin{array}{c}12.0-19.9 \\
(30)\end{array}$ \\
\hline & October & NA & NA & $\begin{array}{c}9.5 \\
(2.2)\end{array}$ & $\begin{array}{c}4.9-15.9 \\
(31)\end{array}$ \\
\hline & November & NA & NA & $\begin{array}{c}7.4 \\
(2.4)\end{array}$ & $\begin{array}{c}3.1-11.3 \\
(30)\end{array}$ \\
\hline & December & $\begin{array}{l}12.8 \\
(1.2)\end{array}$ & $\begin{array}{c}10.4-16.4 \\
(12)\end{array}$ & $\begin{array}{c}6.0 \\
(1.8)\end{array}$ & $\begin{array}{c}3.4-10.7 \\
(12)\end{array}$ \\
\hline \multirow[t]{5}{*}{1988} & January & $\begin{array}{c}9.9 \\
(1.4)\end{array}$ & $\begin{array}{c}5.6-13.4 \\
(25)\end{array}$ & $\begin{array}{c}3.0 \\
(2.7)\end{array}$ & $\begin{array}{c}-(6.8)-4.3 \\
(25)\end{array}$ \\
\hline & February & $\begin{array}{l}11.4 \\
(1.7)\end{array}$ & $\begin{array}{c}7.6-16.5 \\
(29)\end{array}$ & $\begin{array}{c}4.5 \\
(2.4)\end{array}$ & $\begin{array}{c}0.6-10.1 \\
(29)\end{array}$ \\
\hline & March & $\begin{array}{l}13.1 \\
(2.1)\end{array}$ & $\begin{array}{c}8.3-21.8 \\
(31)\end{array}$ & $\begin{array}{c}7.9 \\
(3.9)\end{array}$ & $\begin{array}{c}-(3.6)-18.0 \\
(31)\end{array}$ \\
\hline & April & $\begin{array}{l}16.6 \\
(1.9)\end{array}$ & $\begin{array}{c}12.3-21.9 \\
(30)\end{array}$ & $\begin{array}{c}2.4 \\
(2.7)\end{array}$ & $\begin{array}{c}6.1-19.7 \\
(30)\end{array}$ \\
\hline & May & $\begin{array}{l}20.1 \\
(2.0)\end{array}$ & $\begin{array}{c}14.4-24.6 \\
(31)\end{array}$ & $\begin{array}{l}14.9 \\
(2.8)\end{array}$ & $\begin{array}{c}7.4-20.9 \\
(31)\end{array}$ \\
\hline
\end{tabular}


Table 2.3 (continued)

\begin{tabular}{|c|c|c|c|c|c|}
\hline \multicolumn{2}{|c|}{ Sampling period } & \multicolumn{2}{|c|}{ MIK 0.50} & \multicolumn{2}{|c|}{ GCK 2.4} \\
\hline Year & Month & Mean & Range & Mean & Range \\
\hline & June & $\begin{array}{l}24.3 \\
(1.9)\end{array}$ & $\begin{array}{c}19.1-29.5 \\
(30)\end{array}$ & $\begin{array}{l}18.3 \\
(3.0)\end{array}$ & $\begin{array}{c}11.4-25.3 \\
(30)\end{array}$ \\
\hline & July & $\begin{array}{l}25.3 \\
(1.9)\end{array}$ & $\begin{array}{c}19.6-32.2 \\
(26)\end{array}$ & $\begin{array}{l}20.8 \\
(2.13)\end{array}$ & $\begin{array}{c}14.7-25.6 \\
(26)\end{array}$ \\
\hline & August & $\begin{array}{l}26.7 \\
(1.2)\end{array}$ & $\begin{array}{c}23.1-29.9 \\
(26)\end{array}$ & $\begin{array}{l}21.3 \\
(1.4)\end{array}$ & $\begin{array}{c}16.8-24.1 \\
(26)\end{array}$ \\
\hline & September & $\begin{array}{l}23.9 \\
(1.3)\end{array}$ & $\begin{array}{c}20.4-28.3 \\
(30)\end{array}$ & $\begin{array}{l}18.3 \\
(2.0)\end{array}$ & $\begin{array}{c}2.7-23.5 \\
(30)\end{array}$ \\
\hline & October & $\begin{array}{l}18.3 \\
(2.3)\end{array}$ & $\begin{array}{c}13.6-24.9 \\
(31)\end{array}$ & $\begin{array}{l}10.3 \\
(3.7)\end{array}$ & $\begin{array}{c}2.6-21.4 \\
(31)\end{array}$ \\
\hline & November & $\begin{array}{l}14.7 \\
(1.5)\end{array}$ & $\begin{array}{c}10.8-19.1 \\
(30)\end{array}$ & $\begin{array}{c}8.8 \\
(2.0)\end{array}$ & $\begin{array}{c}4.1-13.1 \\
(30)\end{array}$ \\
\hline & December & $\begin{array}{l}11.3 \\
(1.2)\end{array}$ & $\begin{array}{c}5.8-14.5 \\
(31)\end{array}$ & $\begin{array}{c}3.7 \\
(2.7)\end{array}$ & $\begin{array}{c}-(0.2)-10.3 \\
(31)\end{array}$ \\
\hline \multirow[t]{7}{*}{1989} & January & $\begin{array}{l}10.6 \\
(1.3)\end{array}$ & $\begin{array}{c}5.7-14.2 \\
(20)\end{array}$ & $\begin{array}{c}7.2 \\
(1.8)\end{array}$ & $\begin{array}{c}2.5-10.7 \\
(20)\end{array}$ \\
\hline & February & $\begin{array}{l}10.9 \\
(2.6)\end{array}$ & $\begin{array}{c}5.9-16.6 \\
(16)\end{array}$ & $\begin{array}{c}7.3 \\
(2.2)\end{array}$ & $\begin{array}{c}1.7-13.8 \\
(28)\end{array}$ \\
\hline & March & NA & NA & $\begin{array}{l}10.2 \\
(2.5)\end{array}$ & $\begin{array}{c}4.9-18.1 \\
(31)\end{array}$ \\
\hline & April & NA & NA & $\begin{array}{l}12.1 \\
(2.5)\end{array}$ & $6.0-18.4$ \\
\hline & May & $\begin{array}{l}19.5 \\
(1.7)\end{array}$ & $\begin{array}{c}15.1-24.2 \\
(18)\end{array}$ & $\begin{array}{l}14.2 \\
(2.4)\end{array}$ & $\begin{array}{c}9.0-20.9 \\
(26)\end{array}$ \\
\hline & June & $\begin{array}{l}20.2 \\
(1.3)\end{array}$ & $\begin{array}{c}17.5-25.4 \\
(30)\end{array}$ & $\begin{array}{l}16.7 \\
(1.03)\end{array}$ & $\begin{array}{c}14.1-20.8 \\
(30)\end{array}$ \\
\hline & July & $\begin{array}{l}21.4 \\
(1.7)\end{array}$ & $\begin{array}{c}19.4-27.8 \\
(31)\end{array}$ & $\begin{array}{l}17.6 \\
(1.1)\end{array}$ & $\begin{array}{c}14.4-20.1 \\
(31)\end{array}$ \\
\hline
\end{tabular}


Table 2.3 (continued)

\begin{tabular}{|c|c|c|c|c|c|}
\hline \multicolumn{2}{|c|}{ Sampling period } & \multicolumn{2}{|c|}{ MIK 0.50} & \multicolumn{2}{|c|}{$\begin{array}{r}\text { GCK } 2.4 \\
\end{array}$} \\
\hline Year & Month & Mean & Range & Mean & Range \\
\hline & August & $\begin{array}{l}23.3 \\
(0.9)\end{array}$ & $\begin{array}{c}21.0-27.3 \\
(28)\end{array}$ & $\begin{array}{l}17.8 \\
(1.5)\end{array}$ & $\begin{array}{c}13.1-21.5 \\
(28)\end{array}$ \\
\hline & September & NA & NA & $\begin{array}{l}17.5 \\
(1.8)\end{array}$ & $\begin{array}{c}12.8-20.9 \\
(30)\end{array}$ \\
\hline & October & NA & NA & $\begin{array}{l}13.1 \\
(2.8)\end{array}$ & $\begin{array}{c}7.1-18.5 \\
(31)\end{array}$ \\
\hline & November & NA & NA & $\begin{array}{l}10.3 \\
(2.0)\end{array}$ & $\begin{array}{c}5.3-14.3 \\
(30)\end{array}$ \\
\hline & December & NA & NA & $\begin{array}{c}4.9 \\
(2.4)\end{array}$ & $\begin{array}{c}0.4-9.8 \\
(28)\end{array}$ \\
\hline \multirow[t]{8}{*}{1990} & January & $\begin{array}{l}10.5 \\
(1.0)\end{array}$ & $\begin{array}{c}8.0-12.5 \\
(12)\end{array}$ & $\begin{array}{c}7.5 \\
(1.6)\end{array}$ & $\begin{array}{c}2.9-11.1 \\
(31)\end{array}$ \\
\hline & February & $\begin{array}{l}11.6 \\
(1.5)\end{array}$ & $\begin{array}{c}7.7-16.7 \\
(28)\end{array}$ & $\begin{array}{c}9.4 \\
(1.7)\end{array}$ & $\begin{array}{c}4.6-12.8 \\
(28)\end{array}$ \\
\hline & March & $\begin{array}{l}13.1 \\
(1.8)\end{array}$ & $\begin{array}{c}9.2-18.1 \\
(31)\end{array}$ & $\begin{array}{l}10.7 \\
(2.1)\end{array}$ & $\begin{array}{c}5.9-16.6 \\
(31)\end{array}$ \\
\hline & April & $\begin{array}{l}15.7 \\
(2.6)\end{array}$ & $\begin{array}{c}9.8-24.1 \\
(30)\end{array}$ & $\begin{array}{l}12.7 \\
(1.8)\end{array}$ & $\begin{array}{c}10.0-16.6 \\
(2)\end{array}$ \\
\hline & May & $\begin{array}{l}18.3 \\
(2.0)\end{array}$ & $\begin{array}{c}13.2-23.1 \\
(29)\end{array}$ & $\begin{array}{l}14.7 \\
(1.5)\end{array}$ & $\begin{array}{c}10.4-19.2 \\
(29)\end{array}$ \\
\hline & June & $\begin{array}{l}22.1 \\
(2.0)\end{array}$ & $\begin{array}{c}17.6-31.1 \\
(30)\end{array}$ & $\begin{array}{l}16.4 \\
(1.3)\end{array}$ & $\begin{array}{c}12.2-20.8 \\
(30)\end{array}$ \\
\hline & July & $\begin{array}{l}24.1 \\
(1.3)\end{array}$ & $\begin{array}{c}21.4-2.0 \\
(31)\end{array}$ & $\begin{array}{l}18.2 \\
(1.1)\end{array}$ & $\begin{array}{c}15.3-22.0 \\
(31)\end{array}$ \\
\hline & August & $\begin{array}{l}24.2 \\
(1.2)\end{array}$ & $\begin{array}{c}20.8-28.8 \\
(31)\end{array}$ & $\begin{array}{l}18.9 \\
(1.23)\end{array}$ & $\begin{array}{c}16.1-23.3 \\
(31)\end{array}$ \\
\hline
\end{tabular}

${ }^{a} \mathrm{ND}=$ no data available.

Note: Data were obtained with a Ryar Tempmentor digital temperature recorder with values recorded every $20 \mathrm{~min}$, April-June 1987 and every 60 min beginning July 1987. 
Table 24. Location and description of the eight sites on Mitchell Branch that were sampled in various tasks of the Oak Ridge K-25 Site Biological

Monitoring and Abatement Program

\begin{tabular}{|c|c|c|c|c|c|}
\hline \multirow[b]{3}{*}{ Site $^{a}$} & \multirow[b]{3}{*}{ Location $^{b}$} & \multicolumn{4}{|c|}{ Task } \\
\hline & & \multirow{2}{*}{$\begin{array}{l}\text { Toxicity } \\
\text { monitoring }\end{array}$} & \multirow[b]{2}{*}{ Bioaccumulation } & \multicolumn{2}{|c|}{ Community studies } \\
\hline & & & & Benthos & Fish \\
\hline MIK 0.12 & $\begin{array}{l}\text { NPDES monitoring } \\
\text { station } \mathrm{K}-1700\end{array}$ & $\mathbf{X}$ & $\mathbf{X}^{c}$ &.$v s^{d}$ & NS \\
\hline MIK 0.45 & Below storm drain $190(45 \mathrm{~m})$ & $\mathbf{X}$ & NS & $\mathbf{X}$ & $\mathbf{X}$ \\
\hline MIK 0.54 & $\begin{array}{l}\text { Below K-1407-B effluent and } \\
\text { storm drain } 180(90 \mathrm{~m})^{e}\end{array}$ & $\mathrm{X}$ & NS & $\mathrm{X}$ & $\mathbf{X}$ \\
\hline MIK 0.71 & Below storm drain $170(50 \mathrm{~m})$ & $\mathbf{X}$ & NS & $\mathbf{X}$ & $\mathbf{X}$ \\
\hline MIK 0.78 & Above storm drain $170(20 \mathrm{~m})$ & NS & NS & $\mathbf{X}$ & $\mathbf{X}$ \\
\hline MIK 0.86 & $\begin{array}{l}\text { Above storm drain } 170(100 \mathrm{~m}) \\
\text { near storage yard }\end{array}$ & NS & NS & $\mathbf{X}$ & NS \\
\hline MIK 1.0 & $\begin{array}{l}\text { Above storm drain } 170(240 \mathrm{~m}) \\
\text { just downstream of Stoner } \mathrm{Rd}\end{array}$ & $\mathbf{X}$ & NS & NS & NS \\
\hline MIK 1.43 & $\begin{array}{l}\text { Above storm drain } 170(650 \mathrm{~m}) \\
\text { and the Oak Ridge K-25 Site }\end{array}$ & $\mathbf{X}$ & 8 & $\mathbf{X}$ & $\mathbf{X}$ \\
\hline
\end{tabular}

${ }^{a} \mathrm{MIK}=$ Mitchell Branch kilometer; refers to the distance (in kilometers) above the confluence of Mitchell Branch with Poplar Creek.

${ }^{b}$ Distance above/below storm drain is given in parentheses.

'Sampling was also conducted in Poplar Creek at PCK 6.9, $\sim 300 \mathrm{~m}$ below the mouth of Mitchell Branch. At MIK 0.12, bioaccumulation began and continued throughout a reach which is actually designated as MIK 0.2 .

¿NS = not sampled.

'Effluent from the K-1407-B holding pond and storm drain 180 join just below the pond to form a single discharge to Mitchell Branch.

Reference (control) site.

8Other reference sites were sampled (see Fig. 2.2).

on a storage yard located immediately northeast of Mitchell Branch and (2) minor inputs from several storm drains located further upstream. The sampling sites for the toxicity monitoring and community studies tasks overlap; these tasks/subtasks share four sites, and each includes at least five of the eight primary sites. 


\title{
3. TOXICITY MONITORING
}

\author{
L. A. Kszos
}

The toxicity monitoring task outlined in the BMAP for Mitchell Branch (Loar et al. 1991) included three subtasks. The goals of the task were to (1) monitor ambient water toxicity (subtask 1a), (2) measure the toxicity of selected effluents (subtask 1b), and (3) determine point sources of toxicity (subtask 1c). Results of subtasks $1 \mathrm{~b}$ and $1 \mathrm{c}$ are discussed in Sect. 3.1; results of subtask 1a are discussed in Sect. 3.2.

\subsection{EFFLUENT TOXICITY}

\subsubsection{Introduction}

The EPA supports the use of test organisms to determine the chronic toxicity of a test water (Horning and Weber 1985). As required under the modification of the Oak Ridge K-25 Site NPDES permit (EPA 1986), the toxicity of effluents discharging to Mitchell Branch were evaluated by using the 7-d fathead minnow (Pimephales promelas) larval survival and growth test and the 7-d Ceriodaphnia survival and reproduction test. These two tests are described in detail by Horning and Weber (1985). These tests are static renewal tests, which means that the test solutions are replaced daily for each species.

In October 1988, the K-1407-B pond (and its discharge point) was closed and replaced with the $\mathrm{K}-1407-\mathrm{E}$ pond, the $\mathrm{K}-1407-\mathrm{F}$ pond, and the CNF. Because the K-1407-E and K-1407-F ponds are filled and discharged alternately, they will be discussed as one discharge (K-1407-E/F). Effluent from the CNF (which includes effluent from the TSCA Incinerator) discharges through the K-1407-J basin. Until September 1989, the K-1407-J basin discharged to Mitchell Branch via SD 170. Subsequently, the basin discharged to Poplar Creek. Because the K-1407-E/F pond and the K-1407-J basin are NPDES monitoring points, they were evaluated most extensively.

The three major storm drains that discharge to Mitchell Branch were monitored because (1) they discharge into a reach of Mitchell Branch that is known to be stressed (Smith et al. 1993);

(2) toxicity tests conducted during the first year of the BMAP indicated that the effluents were toxic to fathead minnows and Ceriodaphnia; and (3) after the K-1407-B pond was closed, SDs 170 and 180 were the conduits for effluent from K-1407-E and K-1407-F ponds respectively. Sources of water in the storm drains are as follows: SD 170, which includes K-1407-E pond effluent, K-1407-J basin effluent (until September 1989), oncethrough cooling water, cooling tower blowdown, roof drains, area runoff, and groundwater; SD 180, which includes K-1407-F pond effluent, groundwater, garage area runoff, K-1405 sink drains, K-1401 floor drains, roof drains, oncethrough cooling water, and area runoff; and SD 190, which includes K-1401 floor drains, roof drains, once-through cooling water, effluent from K-1045-A Fire Training Facility, and area runoff (Scheib 1987). 


\subsubsection{Materials and Methods}

Toxicity tests with effluents from the K-1407-B pond, K-1407-E/F pond, K-1407-J basin, SDs 170,180 , and 190 were conducted by using the fathead minnow and Ceriodaphnia dubia chronic toxicity tests described in Sect. 3.1.1. The K-1407-B pond effluent was evaluated every other month from October 1986 to April 1988. Results of the tests conducted with effluent from K-1407-B pond during 1986-87 are discussed in Smith et al. (1993). A summary of all the tests conducted to date is provided in Sect. 3.1.2.1. The K-1407-E/F pond effluent was evaluated about every other month from April 1988 to June 1990. The K-1407-J basin effluent was evaluated every other month from December 1988 to June 1990. The number of toxicity tests used to evaluate storm drain effluents during the same period were, for SD 170, eight; for SD 180, six; and for SD 190, six.

For each effluent toxicity test, seven daily grab samples were taken at the point of discharge. All samples were delivered to the Aquatic Toxicology Laboratory at ORNL by personnel of the Oak Ridge K-25 Site via chain-of-custody procedures (Kszos et al. 1989). Time of collection, water temperature, and arrival time in the laboratory were recorded. Upon arrival in the laboratory, the water was warmed or cooled to $25^{\circ} \mathrm{C}$ and dilutions were made if necessary. Tests with the two species were usually conducted concurrently. On each day of a test, subsamples of each effluent or water sample were routinely analyzed for $\mathrm{pH}$, conductivity, alkalinity, water hardness, total residual chlorine (TRC), and free chlorine (Kszos et al. 1988). Other chemical measurements were made by the Oak Ridge K-25 Site Process Support Department.

Dechlorination of the effluents from SDs 170,180 , and 190 were frequently used to evaluate the contribution of TRC to toxicity. Dechlorination was accomplished by adding $0.1 \mathrm{~N}$ sodium thiosulfate dropwise to the effluent until TRC was $0.0 \mathrm{mg} / \mathrm{L}$.

SAS statistical software [Statistical Analysis System for personal computers (PC-SAS), release 6.02] was used to analyze all data. Survival percentages for fathead minnow larvae and for Ceriodaphnia were transformed (arsine squareroot; Steel and Torrie 1960) before being analyzed. All fecundity values are for females that survived all $7 \mathrm{~d}$ of the test. Significant reductions in Ceriodaphnia survival and fecundity (compared with a control) were determined by using Fisher's Exact Test and Dunnett's Procedure, respectively (Horning and Weber 1985). Significant reductions in fathead minnow survival and growth (compared with a control) were determined by using Dunnett's Procedure (Horning and Weber 1985). Dunnett's Procedure yields the least significant difference and the no-observed-effect concentration (NOEC). Unless noted otherwise, statements of significance are based on $p<0.05$.

\subsubsection{Results}

\subsubsection{K-1407-B Pond}

A summary of all the toxicity tests and concurrent water quality measurements for tests conducted during $1987-88$ are provided in Table 3.1. Results of the tests conducted during 1986-87 are discussed in Smith et al. 1993 and provided here for comparison with those from the K-1407-E/F pond. The effluent's NOECs for the two tests conducted in 1988 were $100 \%$ for both species. Water quality during the two tests was similar to that in 1986-87. 
Table 3.1. Summary of toxicity test results and mean ( \pm 1 SD) water quality parameters $(n=\eta)$ for effluent from the K-1407-B pond

\begin{tabular}{|c|c|c|c|c|c|}
\hline \multirow{2}{*}{$\begin{array}{l}\text { Test } \\
\text { Period }\end{array}$} & \multicolumn{2}{|c|}{ No-observed-effect concentration (\%) } & \multirow[b]{2}{*}{ Alkalinity ${ }^{b}$} & \multirow[b]{2}{*}{ Hardness $^{b}$} & \multirow[b]{2}{*}{ Conductivity } \\
\hline & Fathead minnow & Ceriodaphnia & & & \\
\hline Oct 1986 & 100 & $<100$ & $143(25)$ & $180(47)$ & $441(74)$ \\
\hline Dec 1986 & 100 & $<20$ & $66(8)$ & $725(124)$ & $2260(167)$ \\
\hline Feb 1987 & 60 & $<20$ & $36(21)$ & $525(110)$ & $1465(261)$ \\
\hline Mar 1987 & $\mathrm{NT}^{d}$ & $<50$ & $78(12)$ & $599(235)$ & $2464(995)$ \\
\hline Apr 1987 & 100 & $<100$ & $61(12)$ & $701(168)$ & $1967(256)$ \\
\hline Jun 1987 & $<100$ & 100 & $56(14)$ & $310(18)$ & $1353(184)$ \\
\hline Jul $1987^{e}$ & NT & 100 & $60(0)$ & $445(0)$ & $1610(0)$ \\
\hline Aug 1987 & $<50$ & 50 & $44(3)$ & $510(120)$ & 2295 (1159) \\
\hline Oct 1987 & 100 & $<50$ & $70(8)$ & $453(41)$ & 1657 (67) \\
\hline Dec 1987 & 100 & $<100$ & $72(8)$ & $410(23)$ & $1572(120)$ \\
\hline Feb 1988 & 100 & 100 & $97(28)$ & $455(763$ & $3061(427)$ \\
\hline Apr 1988 & 100 & 100 & $97(16)$ & $502(76)$ & $1805(294)$ \\
\hline
\end{tabular}

${ }^{a}$ The no-observed-effect concentration designates the highest concentration of the effluent tested causing no significant $(p>0.05)$ reduction in survival or growth of fathead minnow larvae, or survival or reproduction of Ceriodaphnia.

${ }^{b} \mathrm{mg} / \mathrm{L} \mathrm{CaCO}_{3}$.

${ }^{\mathrm{c}} \boldsymbol{\mathrm { S }} / \mathrm{cm}$.

NT $=$ not tested.

Test used one grab sample.

\subsubsection{K-1407-E/F Pond}

The results of the toxicity tests and concurrent water quality measurements are summarized in Table 3.2. From April 1988 to June 1990, effluent from K-1407-E/F pond was tested 14 times with fathead minnows and Ceriodaphnia; however, 2 of the Ceriodaphnia tests were invalid because of low fecundity in the controls. Results of these toxicity tests are summarized in terms of the NOEC of the effluents (Table 3.2). The effluent NOEC for fathead minnows was never less than $100 \%$, whereas the effluent NOEC for Ceriodaphnia ranged from 6 to $100 \%$. The NOEC was $100 \%$ in only 4 of the 12 valid Ceriodaphnia tests.

Effluent from K-1407-E/F pond was characterized by periods of high conductivity and hardness (Table 3.2). During the April 1988 to June 1990 tests, pH, mean alkalinity, mean hardness, and mean conductivity ranged from 7.53 to $10.17,37$ to $93 \mathrm{mg} / \mathrm{L}, 343$ to $741 \mathrm{mg} / \mathrm{L}$, and 854 to $3695 \mu \mathrm{S} / \mathrm{cm}$ respectively.

Results of chemical analyses obtained concurrently with some of the toxicity tests by the Oak Ridge K-25 Site Process Support Department are summarized in Table 3.3. Only those constituents that 
Table 3.2. Summary of toxicity test results and mean ( \pm 1 SD) water quality parameters $(n=7)$ for effluent from the K-1407-E/F ponds

\begin{tabular}{|c|c|c|c|c|c|}
\hline \multirow{2}{*}{$\begin{array}{l}\text { Test } \\
\text { Period }\end{array}$} & \multicolumn{2}{|c|}{ No-observed-effect concentration $(\%)^{a}$} & \multirow[b]{2}{*}{ Alkalinity ${ }^{b}$} & \multirow[b]{2}{*}{ Hardness $^{b}$} & \multirow[b]{2}{*}{ Conductivity } \\
\hline & Fathead minnow & Ceriodaphnia & & & \\
\hline Apr 1988 & 100 & 100 & $68(12)$ & $538(162)$ & $2204(425)$ \\
\hline Jun 1988 & 100 & 100 & $71(21)$ & $343(103)$ & $1655(365)$ \\
\hline Aug 1988 & 100 & 100 & $68(11)$ & $535(151)$ & $2355(1856)$ \\
\hline Oct 1988 & 100 & $\mathbf{l}^{d}$ & $75(5)$ & $722(106)$ & $2838(331)$ \\
\hline Dec 1988 & 100 & I & $75(3)$ & $764(77)$ & $2761(131)$ \\
\hline Feb 1989 & 100 & 50 & $60(5)$ & $445(88)$ & $1610(335)$ \\
\hline Apr 1989 & 100 & $<25$ & $89(23)$ & $475(141)$ & $1466(335)$ \\
\hline Jun 1989 & 100 & 100 & $68(9)$ & $297(68)$ & 854 (195) \\
\hline Aug 1989 & 100 & 25 & $66(7)$ & $413(102)$ & $1864(619)$ \\
\hline Oct 1989 & 100 & 12 & $93(11)$ & $398(72)$ & $1686(286)$ \\
\hline Dec 1989 & 100 & $<6$ & $69(8)$ & 703 (155) & $2112(213)$ \\
\hline Feb 1990 & 100 & 25 & $76(2)$ & $434(93)$ & $2210(543)$ \\
\hline Apr 1990 & 100 & 6 & $59(8)$ & 741 (132) & $2265(236)$ \\
\hline Jun 1990 & 100 & 6 & $37(10)$ & $814(141)$ & $3695(324)$ \\
\hline
\end{tabular}

The no-observed-effect concentration designates the highest concentration of the effluent tested causing no significant $(p>0.05)$ reduction in survival or growth of fathead minnow larvae, or survival or reproduction of Ceriodaphnia.

${ }^{b} \mathrm{mg} / \mathrm{L} \mathrm{CaCO}{ }_{3}$.

${ }^{\mathrm{c}} \boldsymbol{\mathrm { S }} / \mathrm{cm}$.

$d \mathrm{I}=$ invalid test because of low fecundity.

had concentrations consistently above detection levels or that were of toxicological concern were included; complete data sets are available elsewhere (McGaha 1989a, 1989b, 1989c, 1989d; Shoemaker et al. 1990). High concentrations of $\mathrm{Ca}$, $\mathrm{Cl}$, dissolved solids, $\mathrm{Na}$, and $\mathrm{SO}_{4}$ are discharged from the K-1407-E/F ponds; the maximum concentrations of these substances measured during the toxicity tests were $250 \mathrm{mg} / \mathrm{L}, 714 \mathrm{mg} / \mathrm{L}, 2048 \mathrm{mg} / \mathrm{L}$, $440 \mathrm{mg} / \mathrm{L}$, and $693 \mathrm{mg} / \mathrm{L}$ respectively. Temporally, the concentrations of $\mathrm{Ca}, \mathrm{Cl}$, dissolved solids, $\mathrm{Na}$, and $\mathrm{SO}_{4}$ were quite variable and corresponded with patterns of conductivity and hardness. The concentrations of most other substances varied less from test to test, with a few notable exceptions: (1) aluminum was elevated in the August 1988, February 1989, and June 1989 tests; (2) iron was elevated in the June and October 1989 tests; (3) manganese was elevated in the February 1989 test; and (4) nickel was elevated in the October 1989 test.

The concentrations of chemicals in $\mathrm{K}-1407-\mathrm{E} / \mathrm{F}$ pond effluent were very similar to those found in the K-1407-B pond effluent (Smith et al. 1993). For example, the $\mathrm{K}-1407-\mathrm{B}$ pond and $\mathrm{K}-1407-\mathrm{E} / \mathrm{F}$ pond 
Table 3.3. Mean (range in parentheses) concentrations of selected parameters (in milligrams per liter) in the efiluents from the K-1407-E/F ponds obtained by the Oak Ridge K-25 Site Process Support Department in conjunction with the toricity tests

\begin{tabular}{|c|c|c|c|c|c|c|c|}
\hline Analysis & Aug 1988 & Oct 1988 & Dec 1988 & Feb 1989 & Apr 1989 & Jun 1989 & Oct 1989 \\
\hline Aluminum & $\begin{array}{c}0.41 \\
(0.18-0.74)\end{array}$ & $\begin{array}{c}0.03 \\
\left(\mathrm{BD}^{2}-0.06\right)\end{array}$ & $\begin{array}{c}0.18 \\
(B D-0.78)\end{array}$ & $\begin{array}{c}0.61 \\
(B D-1.2)\end{array}$ & $\begin{array}{c}0.18 \\
(B D-0.43)\end{array}$ & $\begin{array}{c}0.52 \\
(0.14-1.1)\end{array}$ & $\begin{array}{c}0.04 \\
(0.14-0.57)\end{array}$ \\
\hline Barium & $\begin{array}{c}0.02 \\
(0.007-0.04)\end{array}$ & $\begin{array}{c}0.01 \\
(0.004-0.011)\end{array}$ & $\begin{array}{c}0.01 \\
(\mathrm{BD}-0.02)\end{array}$ & $\begin{array}{c}0.03 \\
(B D-0.04)\end{array}$ & $\begin{array}{c}0.02 \\
(0.01-0.03)\end{array}$ & $\begin{array}{c}0.03 \\
(0.03-0.04)\end{array}$ & $\begin{array}{c}0.04 \\
(0.02-0.04)\end{array}$ \\
\hline Boron & $\begin{array}{c}0.12 \\
(0.08-0.16)\end{array}$ & $\begin{array}{c}0.04 \\
(0.03-0.05)\end{array}$ & $\begin{array}{c}0.16 \\
(0.05-0.35\end{array}$ & $\begin{array}{c}0.33 \\
(0.02-0.59)\end{array}$ & $\begin{array}{c}0.06 \\
(0.05-0.08)\end{array}$ & $\begin{array}{c}0.08 \\
(0.06-0.12)\end{array}$ & $\begin{array}{c}0.05 \\
(0.04-0.06)\end{array}$ \\
\hline Calcium & $\begin{array}{c}127 \\
(81-210)\end{array}$ & $\begin{array}{c}190 \\
(170-220)\end{array}$ & $\begin{array}{c}203 \\
(85-250)\end{array}$ & $\begin{array}{c}133 \\
(85-180)\end{array}$ & $\begin{array}{c}157 \\
(76-220)\end{array}$ & $\begin{array}{c}91 \\
(59-120)\end{array}$ & $\begin{array}{c}125 \\
(75-170)\end{array}$ \\
\hline Chloride & $\begin{array}{c}233 \\
(54-465)\end{array}$ & $\begin{array}{c}597 \\
(500-714)\end{array}$ & $\begin{array}{c}514 \\
(456-563)\end{array}$ & $\begin{array}{c}261 \\
(167-419)\end{array}$ & $\begin{array}{c}227 \\
(136-282)\end{array}$ & $\begin{array}{c}91 \\
(32-133)\end{array}$ & $\begin{array}{c}231 \\
(173-324)\end{array}$ \\
\hline $\begin{array}{l}\text { Dissolved } \\
\text { solids }\end{array}$ & $\begin{array}{c}1051 \\
(452-1788)\end{array}$ & $\begin{array}{c}1790 \\
(1570-2048)\end{array}$ & $\begin{array}{c}1888 \\
(1790-1998)\end{array}$ & $\begin{array}{c}1041 \\
(788-1416)\end{array}$ & $\begin{array}{c}1017 \\
(610-1266)\end{array}$ & $\begin{array}{c}599 \\
(414-838)\end{array}$ & $\begin{array}{c}1229 \\
(1160-1470)\end{array}$ \\
\hline Fluoride & $\begin{array}{c}0.3 \\
(0.1-1.0)\end{array}$ & $\begin{array}{c}0.2 \\
(0.2-0.3)\end{array}$ & $\begin{array}{c}0.3 \\
(0.3-0.3)\end{array}$ & $\begin{array}{c}0.2 \\
(0.2-0.2)\end{array}$ & $\begin{array}{c}0.2 \\
(0.2-0.4)\end{array}$ & $\begin{array}{c}0.4 \\
(0.2-0.8)\end{array}$ & $\begin{array}{c}0.2 \\
(B D-0.2)\end{array}$ \\
\hline İron & $\begin{array}{c}0.3 \\
(0.13-0.58)\end{array}$ & $\begin{array}{c}0.2 \\
(0.03-0.27)\end{array}$ & $\begin{array}{c}0.6 \\
(0.2-1.1)\end{array}$ & $\begin{array}{c}0.7 \\
(\mathrm{BD}-1.4)\end{array}$ & $\begin{array}{c}0.4 \\
(0.02-0.96)\end{array}$ & $\begin{array}{c}1.0 \\
(0.3-2.0)\end{array}$ & $\begin{array}{c}1.2 \\
(0.1-2.0)\end{array}$ \\
\hline Magnesium & $\begin{array}{c}11.2 \\
(1.7-30.0)\end{array}$ & $\begin{array}{c}23.4 \\
(20-29)\end{array}$ & $\begin{array}{c}28.3 \\
(13-34)\end{array}$ & $\begin{array}{c}22.9 \\
(14-30)\end{array}$ & $\begin{array}{c}21.1 \\
(14-26)\end{array}$ & $\begin{array}{c}12.0 \\
(9-17)\end{array}$ & $\begin{array}{c}18.4 \\
(14-22)\end{array}$ \\
\hline Manganese & $\begin{array}{c}0.02 \\
(0.008-0.03)\end{array}$ & $\begin{array}{c}0.04 \\
(0.03-0.06)\end{array}$ & $\begin{array}{c}0.10 \\
(0.04-0.14)\end{array}$ & $\begin{array}{c}0.24 \\
(0.13-0.31)\end{array}$ & $\begin{array}{c}0.07 \\
(0.02-0.15)\end{array}$ & $\begin{array}{c}0.07 \\
(0.04-0.10)\end{array}$ & $\begin{array}{c}0.08 \\
(0.04-0.13)\end{array}$ \\
\hline
\end{tabular}


Table 33 (comtinued)

\begin{tabular}{|c|c|c|c|c|c|c|c|}
\hline Analysis & Aug 1988 & Oct 1988 & Dec 1988 & Feb 1989 & Apr 1989 & Jun 1989 & Oct 1989 \\
\hline Nickel & $\mathrm{BD}$ & $\begin{array}{c}0.01 \\
(B D-0.012)\end{array}$ & $\begin{array}{c}0.05 \\
(0.02-0.1)\end{array}$ & BD & $\begin{array}{c}0.06 \\
(B D-0.14)\end{array}$ & $\begin{array}{c}0.02 \\
(0.01-0.04)\end{array}$ & $\begin{array}{c}0.32 \\
(0.10-0.88)\end{array}$ \\
\hline Silicon & $\begin{array}{c}2.5 \\
(1.3-3.0)\end{array}$ & $\begin{array}{c}3.3 \\
(2.8-4.1)\end{array}$ & $\begin{array}{c}4.5 \\
(3.9-7.1)\end{array}$ & $\begin{array}{c}5.3 \\
(2.9-7.3)\end{array}$ & $\begin{array}{c}2.2 \\
(1.7-2.7)\end{array}$ & $\begin{array}{c}3.9 \\
(3.2-4.6)\end{array}$ & $\begin{array}{c}6.9 \\
(5.9-7.4)\end{array}$ \\
\hline Sodium & $\begin{array}{c}150 \\
(48-280)\end{array}$ & $\begin{array}{c}374 \\
(330-440)\end{array}$ & $\begin{array}{c}315 \\
(270-440)\end{array}$ & $\begin{array}{c}192 \\
(130-290)\end{array}$ & $\begin{array}{c}160 \\
(100-190)\end{array}$ & $\begin{array}{c}63 \\
(31-98)\end{array}$ & $\begin{array}{c}197 \\
(140-260)\end{array}$ \\
\hline Sulfate & $\begin{array}{c}385 \\
(138-592)\end{array}$ & $\begin{array}{c}507 \\
(456-581)\end{array}$ & $\begin{array}{c}651 \\
(610-693)\end{array}$ & $\begin{array}{c}372 \\
(201-527)\end{array}$ & $\begin{array}{c}343 \\
(183-479)\end{array}$ & $\begin{array}{c}249 \\
(151-341)\end{array}$ & $\begin{array}{c}381 \\
(208-498)\end{array}$ \\
\hline $\begin{array}{l}\text { Suspended } \\
\text { solids }\end{array}$ & $\begin{array}{c}6.7 \\
(4-14)\end{array}$ & $\begin{array}{c}3.7 \\
(2-6)\end{array}$ & $\begin{array}{c}7.9 \\
(4-21)\end{array}$ & $\begin{array}{c}3.1 \\
(B D-8)\end{array}$ & $\begin{array}{c}6.6 \\
(1-24)\end{array}$ & $\begin{array}{c}11.7 \\
(3-36)\end{array}$ & $\begin{array}{c}10.6 \\
(1-22)\end{array}$ \\
\hline Zinc & $\begin{array}{c}0.14 \\
(0.09-0.22)\end{array}$ & $\begin{array}{c}0.12 \\
(0.03-0.37)\end{array}$ & $\begin{array}{c}0.15 \\
(0.05-0.29)\end{array}$ & $\begin{array}{c}0.27 \\
(0.08-0.57)\end{array}$ & $\begin{array}{c}0.01 \\
(B D-0.03)\end{array}$ & $\begin{array}{c}0.03 \\
(0.02-0.07)\end{array}$ & $\begin{array}{c}0.03 \\
(0.01-0.04)\end{array}$ \\
\hline
\end{tabular}

${ }^{a} \mathrm{BD}=$ below detection

Sources: McGaha, M. A, 1989a, Toxicity Monitoring at ORGDP July-September 1988, Report No. K/QT-288, Oak Ridge Gaseous Diffusion Plant, Oak Ridge, Tennessee; McGaha, M. A, 1989b, Toxicity Monitoning at ORGDP October-December 1988, Report No. K/QT-ח311, Oak Ridge Gaseous Diffusion Plant, Oak Ridge, Tennessee; McGaha, M. A, 1989c, Toxiciny Monitoring at ORGDP January-March 1989, Report No. K/QT-312, Oak Ridge Gaseous Diffusion Plant, Oak Ridge, Tennessee; McGaha, M. A., 1989d, Toxicity Monitoring at ORGDP April-June 1989, Report No. K/QT-313, Oak Ridge Gaseous Diffusion Plant, Oak Ridge, Tennessee; and Shoemaker, J. L., et al., 1990, Toxicity Monitoring at ORGDP October-December 1989, Report No. KJQT-0373, Oak Ridge Gaseous Diffusion Plant, Oak Ridge, Tennessee 
contained similar amounts (average of the mean for each test) of $\mathrm{Al}(0.21 \mathrm{us}$ $0.28 \mathrm{mg} / \mathrm{L}), \mathrm{Ca}(173$ vs $307 \mathrm{mg} / \mathrm{L}), \mathrm{Cl}$ (297 vs $307 \mathrm{mg} / \mathrm{L}), \mathrm{Mg}$ (18.5 vs $19.6 \mathrm{mg} / \mathrm{L})$, and $\mathrm{Na}$ (197.5 vs $207 \mathrm{mg} / \mathrm{L})$.

\subsubsection{K-1407-J Basin}

The results of the toxicity tests and concurrent water quality measurements are summarized in Table 3.4. From December 1988 to June 1990, effluent from K-1407-J basin was tested 11 times with fathead minnows and Ceriodaphnia. Results of these tests are summarized in terms of the effluent NOEC (Table 3.4). One of the Ceriodaphnia tests was invalid because of low fecundity in the controls. A definitive NOEC could not be determined for two additional Ceriodaphnia tests and one fathead minnow test because (1) there was no effect at the highest concentration of effluent tested $(60 \%)$ or (2) the lowest concentration tested (6\%) caused a reduction in survival. The effluent NOEC was $100 \%$ for fathead minnows in nine of ten tests; the NOEC was $<100 \%$ only during the test conducted in April 1990. The effluent NOEC was $100 \%$ for Ceriodaphnia in only two of eight conclusive tests; NOECs for the remaining tests ranged from 1 to $50 \%$.

The effluent periodically had high alkalinity, hardness, and conductivity (Table 3.4). During the December 1988

Table 3.4. Summary of toxicity test results and mean ( \pm 1 SD) water quality parameters $(n=7)$ for eflluent from the K-1407-J basin

\begin{tabular}{|c|c|c|c|c|c|}
\hline \multirow{2}{*}{$\begin{array}{l}\text { Test } \\
\text { Period }\end{array}$} & \multicolumn{2}{|c|}{ No-observed-effect concentration $(\%)^{a}$} & \multirow[b]{2}{*}{ Alkalinity ${ }^{b}$} & \multirow[b]{2}{*}{ Hardness $^{b}$} & \multirow[b]{2}{*}{ Conductivity } \\
\hline & Fathead minnow & Ceriodaphnia & & & \\
\hline Dec 1988 & 100 & $\mathrm{I}^{\mathrm{d}}$ & $444(112)$ & $250(25)$ & $4215(850)$ \\
\hline Feb 1989 & 100 & 12 & $228(30)$ & $284(28)$ & $2744(504)$ \\
\hline Mar 1989 & $>60$ & $>60$ & $174(14)$ & $244(40)$ & $1356(305)$ \\
\hline Apr 1989 & 100 & 50 & $148(59)$ & $332(99)$ & $1450(383)$ \\
\hline Jun 1989 & 100 & 12 & $382(70)$ & $136(19)$ & $8171(1499)$ \\
\hline Aug 1989 & 100 & 12 & $125(30)$ & $1431(423)$ & $4257(1130)$ \\
\hline Oct 1989 & 100 & 6 & $79(8)$ & $402(85)$ & $1148(207)$ \\
\hline Dec 1989 & 100 & $<6$ & $81(7)$ & $452(117)$ & $1148(226)$ \\
\hline Feb 1990 & 100 & 1 & $93(2)$ & $418(70)$ & $1285(223)$ \\
\hline Apr 1990 & 50 & 100 & $177(21)$ & $262(14)$ & $676(101)$ \\
\hline Jun 1990 & 100 & 100 & $461(85)$ & $197(12)$ & $1926(256)$ \\
\hline
\end{tabular}

The no-observed-effect concentration designates the highest concentration of the effluent tested causing no significant $(p>0.05)$ reduction in survival or growth of fathead minnow larvae, or survival or reproduction of Ceriodaphnia.

${ }^{\circ} \mathrm{mg} / \mathrm{L} \mathrm{CaCO}$.

${ }^{\mathrm{c}} \mathrm{S} \mathrm{S} / \mathrm{cm}$.

$\mathrm{I}=$ invalid test because of low fecundity in the control water. 
to June 1990 tests, $\mathrm{pH}$, mean alkalinity, mean hardness, and mean conductivity ranged from 7.67 to $8.88,79$ to $461 \mathrm{mg} / \mathrm{L}$, 140 to $1431 \mathrm{mg} / \mathrm{L}$, and 630 to $8536 \mu \mathrm{S} / \mathrm{cm}$ respectively (Table 3.4 ).

Results of chemical analyses obtained concurrently with the toxicity tests by the Oak Ridge K-25 Site Process Support Department are summarized in Table 3.5. Only those constituents that had concentrations consistently above detection levels or that were of toxicological concern were included; complete data sets are available elsewhere (McGaha 1989a, 1989b, 1989c, 1989d; Shoemaker et al. 1990). High concentrations of $\mathrm{Ca}, \mathrm{Cl}$, dissolved solids, $\mathrm{Na}$, and $\mathrm{SO}_{4}$ were discharged from the pond; releases of these constituents corresponded with patterns of high conductivity and hardness. The highest concentiations of $\mathrm{Ca}, \mathrm{Cl}$, dissolved solids, $\mathrm{Na}$, anci $\mathrm{SO}_{4}$ discharged during the toxicity tests were $240,3470,6632,2200$, and $970 \mathrm{mg} / \mathrm{t}$ respectively. The concentrations of most other substances variet less from test to test, with a few noiahle exceptions: (1) Al and $\mathrm{Zn}$ were elevated in the March 1989 test, (2) Ni was elevated in the April and October 1989 test, and (3) F was elevated in the June 1989 test.

\subsubsection{Storm drains}

Toxicity test results and water quality measurements of the storm drain effluents are summarized in Table 3.6. Daily concentrations of TRC in SD 170 effluent ranged from 0 to $1.78 \mathrm{mg} / \mathrm{L}$; maximum concentrations during each test ranged from 0.22 to $1.78 \mathrm{mg} / \mathrm{L}$. Untreated effluent was toxic (NOEC $<100 \%$ ) to minnows in six of seven tests; dechlorinating the effluent eliminated the toxicity in four of the five tests in which it was attempted. Untreated SD 170 effluent was toxic to Ceriodaphnia in all eight tests; dechlorination eliminated the toxicity in two of the seven tests in which it was attempted.

Daily and maximum concentrations of TRC in SD 180 effluent ranged from 0 to $0.57 \mathrm{mg} / \mathrm{L}$. In four tests (October 1988, July 1989, and January and May 1990), the concentration of TRC was not high enough to cause mortality of fathead minnows or Ceriodaphnia in $7 \mathrm{~d}$ (A. J. Stewart, Environmental Sciences Division, unpublished data). When the effluent contained high concentrations of TRC (0.36 and $0.57 \mathrm{mg} / \mathrm{L})$, it was toxic to both species. Dechlorination of the effluent during one test (August 1988) removed the toxicity.

Daily concentrations of TRC in SD 190 effluent ranged from 0 to $0.99 \mathrm{mg} / \mathrm{L}$; maximum concentrations during each test ranged from 0.06 to $0.99 \mathrm{mg} / \mathrm{L}$. During all tests but one (May 1990), the maximum concentration of TRC was high enough to be toxic to Ceriodaphnia

(A. J. Stewart, Environmental Sciences Division, unpublished data). Untreated SD 190 effluent was toxic to fathead minnows in three of six tests; dechlorination eliminated toxicity to the minnows in one of five of the tests in which it was attempted. Untreated SD 190 effluent was toxic to Ceriodaphnia in five of six tests; dechlorination eliminated the toxicity in three of five of the tests in which it was attempted.

Results of chemical analyses conducted concurrently with the toxicity tests by the Oak Ridge K-25 Site Process Support Department during July 1988 to July 1989 are summarized in Tables 3.7 through 3.9. Effluent from SD 180 typically contained the highest concentrations (compared with SDs 170 and 190) of $\mathrm{Al}, \mathrm{Ba}$, $\mathrm{Ca}, \mathrm{Cl}, \mathrm{F}, \mathrm{Fe}, \mathrm{Mn}, \mathrm{Na}, \mathrm{SO}_{4}$, dissolved solids, and suspended solids. The concentrations of these substances were particularly high in SDs 170 and 180 during the July 1989 test. Nickel was elevated in the July 1988 test of effluent from SD 190. 
Table 3.5. Mean (range in parentheses) concentrations (in milligrams per titex) of selected parmeters for the K-1407-J besin efineent obtained by the Oak Ridge K-25 Site Process Support Departent taken in conjunction with some of the toucicity tests

\begin{tabular}{|c|c|c|c|c|c|c|}
\hline Analysis & Dec 1988 & Feb 1989 & Mar 1989 & Apr 1989 & Jun 1989 & Oct 1989 \\
\hline Aluminum & $\begin{array}{c}0.7 \\
\left(\mathrm{BD}^{a}-1.2\right)\end{array}$ & $\begin{array}{c}0.7 \\
\text { (BD-1.7) }\end{array}$ & $\begin{array}{c}1.1 \\
(0.75-1.4)\end{array}$ & $\begin{array}{c}0.2 \\
(0.07-0.41)\end{array}$ & $\begin{array}{c}0.7 \\
(0.51-1.0)\end{array}$ & $\begin{array}{c}0.7 \\
(0.19-1.9)\end{array}$ \\
\hline Calcium & $\begin{array}{c}94 \\
(41-240)\end{array}$ & $\begin{array}{c}90 \\
(79-100)\end{array}$ & $\begin{array}{c}65 \\
(52-86)\end{array}$ & $\begin{array}{c}94 \\
(70-150)\end{array}$ & $\begin{array}{c}39 \\
(31-56)\end{array}$ & $\begin{array}{c}133 \\
(60-180)\end{array}$ \\
\hline Chloride & $\begin{array}{c}730 \\
(378-976)\end{array}$ & $\begin{array}{c}311 \\
(126-395)\end{array}$ & $\begin{array}{c}129 \\
(106-222)\end{array}$ & $\begin{array}{c}191 \\
(81-329)\end{array}$ & $\begin{array}{c}2405 \\
(1520-3470)\end{array}$ & $\begin{array}{c}129 \\
(73-258)\end{array}$ \\
\hline Dissolved solids & $\begin{array}{c}2695 \\
(1640-3348)\end{array}$ & $\begin{array}{c}1862 \\
(1140-2094)\end{array}$ & $\begin{array}{c}880 \\
(746-1296)\end{array}$ & $\begin{array}{c}920 \\
(554-1320)\end{array}$ & $\begin{array}{c}4900 \\
(3278-6632)\end{array}$ & $\begin{array}{c}894 \\
(700-1170)\end{array}$ \\
\hline Fluoride & $\begin{array}{c}33.3 \\
(15-50)\end{array}$ & $\begin{array}{c}22.3 \\
(10-28)\end{array}$ & $\begin{array}{c}11.1 \\
(8-20)\end{array}$ & $\begin{array}{c}3.9 \\
(2.6-4.6)\end{array}$ & $\begin{array}{c}40.7 \\
(30-55)\end{array}$ & $\begin{array}{c}0.9 \\
(0.8-1.1)\end{array}$ \\
\hline Iron & $\begin{array}{c}0.8 \\
(0.19-1.2)\end{array}$ & $\begin{array}{c}0.6 \\
(0.31-1.1)\end{array}$ & $\begin{array}{c}0.6 \\
(0.34-1.2)\end{array}$ & $\begin{array}{c}0.4 \\
(0.13-0.77)\end{array}$ & $\begin{array}{c}0.6 \\
(0.2-1.0)\end{array}$ & $\begin{array}{c}0.2 \\
(B D-1.2)\end{array}$ \\
\hline Magnesium & $\begin{array}{c}14.1 \\
(9-33)\end{array}$ & $\begin{array}{c}9.7 \\
(8-10)\end{array}$ & $\begin{array}{c}18.7 \\
(11-27)\end{array}$ & $\begin{array}{c}17.9 \\
(14-23)\end{array}$ & $\begin{array}{c}4.5 \\
(4-6)\end{array}$ & $\begin{array}{c}10.6 \\
(6-13)\end{array}$ \\
\hline Manganese & $\begin{array}{c}0.05 \\
(0.04-0.07)\end{array}$ & $\begin{array}{c}0.15 \\
(0.12-0.20)\end{array}$ & $\begin{array}{c}0.02 \\
(B D-0.03)\end{array}$ & $\begin{array}{c}0.04 \\
(0.02-0.07)\end{array}$ & $\begin{array}{c}0.03 \\
(0.03-0.04)\end{array}$ & $\begin{array}{c}0.04 \\
(0.01-0.07)\end{array}$ \\
\hline Nickel & $\begin{array}{c}0.07 \\
(0.02-0.11)\end{array}$ & BD & BD & $\begin{array}{c}0.27 \\
(B D-0.05)\end{array}$ & $\begin{array}{c}0.05 \\
(0.02-0.09)\end{array}$ & $\begin{array}{c}0.22 \\
(0.06-0.47)\end{array}$ \\
\hline Silicon & $\begin{array}{c}6.0 \\
(2.3-8.9)\end{array}$ & $\begin{array}{c}8.2 \\
(5.5-11.0)\end{array}$ & $\begin{array}{c}5.1 \\
(4.1-7.0)\end{array}$ & $\begin{array}{c}1.9 \\
(1.5-2.3)\end{array}$ & $\begin{array}{c}6.6 \\
(5.3-8.3)\end{array}$ & $\begin{array}{c}2.9 \\
(1.3-4.8)\end{array}$ \\
\hline
\end{tabular}


Table 35 (continued)

\begin{tabular}{|c|c|c|c|c|c|c|}
\hline Analysis & Dec 1988 & Feb 1989 & Mar 1989 & Apr 1989 & Jun 1989 & Oct 1989 \\
\hline Sodium & $\begin{array}{c}654 \\
(40-970)\end{array}$ & $\begin{array}{c}554 \\
(290-630)\end{array}$ & $\begin{array}{c}228 \\
(180-410)\end{array}$ & $\begin{array}{c}188 \\
(97-260)\end{array}$ & $\begin{array}{c}1671 \\
(1200-2200)\end{array}$ & $\begin{array}{c}92 \\
(32-110)\end{array}$ \\
\hline Sulfate & $\begin{array}{c}667 \\
(473-970)\end{array}$ & $\begin{array}{c}679 \\
(488-762)\end{array}$ & $\begin{array}{c}280 \\
(230-406)\end{array}$ & $\begin{array}{c}289 \\
(175-429)\end{array}$ & $\begin{array}{c}464 \\
(315-611)\end{array}$ & $\begin{array}{c}398 \\
(275-656)\end{array}$ \\
\hline Suspended Solids & $\begin{array}{c}18.6 \\
(9-25)\end{array}$ & $\begin{array}{c}13.4 \\
(4-46)\end{array}$ & $\begin{array}{c}10.0 \\
(230-406)\end{array}$ & $\begin{array}{c}6.6 \\
(4-9)\end{array}$ & $\begin{array}{c}14.4 \\
(8-23)\end{array}$ & $\begin{array}{c}13.6 \\
(3-32)\end{array}$ \\
\hline Zinc & $\begin{array}{c}0.26 \\
(0.02-0.45)\end{array}$ & $\begin{array}{c}0.37 \\
(0.11-1.1)\end{array}$ & $\begin{array}{c}0.48 \\
(0.17-0.78)\end{array}$ & $\begin{array}{c}0.01 \\
(0.01-0.02)\end{array}$ & $\begin{array}{c}0.05 \\
(0.04-0.06)\end{array}$ & $\begin{array}{c}0.06 \\
(0.04-0.08)\end{array}$ \\
\hline
\end{tabular}

$\triangle \mathrm{BD}=$ below detection.

Sources: McGaha, M. A, 1989a, Toxicity Monitoring at ORGDP Juty-September 1988, Report No. K/QT-288, Oak Ridge Gaseous Diffusion Plant, Oak Ridge, Tennessee; McGaha, M. A, 1989b, Toxiciny Monitoring at ORGDP October-December 1988, Report No. K/QT-0311, Oak Ridge Gaseous Diffusion Plant, Oak Ridge, Tennessee; McGaha, M. A, 1989c, Toxicity Monitoring at ORGDP January-March 1989, Report No. K/QT-312, Oak Ridge Gaseous Diffusion Plant, Oak Ridge, Tennessee; McGaha, M. A, 1989d, Toxicity Monitoring at ORGDP April-June 1989, Report No. K/QT-313, Oak Ridge Gaseous Diffusion Plant, Oak Ridge, Tennessee; and Shoemaker, J. L., et al., 1990, Toxicity Monitoring at ORGDP October-December 1989, Report No. K/QT-0373, Oak Ridge Gaseous Diffusion Plant, Oak Ridge, Tennessee 
Table 3.6. Summary of toxicity test results and concentration (in milligrams per liter) of total residual chlorine (TRC) in the storm drain eflluents

\begin{tabular}{|c|c|c|c|c|}
\hline \multirow{2}{*}{$\begin{array}{l}\text { Storm } \\
\text { drain }\end{array}$} & \multirow{2}{*}{$\begin{array}{c}\text { Test } \\
\text { period }\end{array}$} & \multicolumn{2}{|c|}{ No-observed-effect concentration ${ }^{a}(\%)$} & \multirow[b]{2}{*}{ Range TRC } \\
\hline & & Fathead minnow & Ceriodaphnia & \\
\hline \multirow[t]{15}{*}{170} & Jul 1988 & 25 & $<25$ & $0.04-1.65$ \\
\hline & Aug 1988 & $<100$ & $<100$ & $0.00-0.47$ \\
\hline & dechlor. & 100 & $<100$ & \\
\hline & Oct 1988 & $<100$ & $<100$ & $0.00-1.50$ \\
\hline & dechlor. & $<100$ & $<100$ & \\
\hline & Dec 1988 & $<100$ & $<100$ & $0.90-1.78$ \\
\hline & dechlor. & 100 & 100 & \\
\hline & Feb 1989 & NTt & $<100$ & $0.11-1.10$ \\
\hline & dechlor. & NT & $<100$ & \\
\hline & Jul 1989 & 100 & $<100$ & $0.08-0.22$ \\
\hline & dechlor. & 100 & $<100$ & \\
\hline & Jan 1990 & $<100$ & $<100$ & $0.00-0.64$ \\
\hline & dechlor. & 100 & $<100$ & \\
\hline & May 1990 & $<100$ & $<100$ & $0.00-0.69$ \\
\hline & dechlor. & 100 & 100 & \\
\hline \multirow[t]{7}{*}{180} & Jul 1988 & 50 & 50 & $0.00-0.36$ \\
\hline & Aug 1988 & $<100$ & $<100$ & $0.00-0.57$ \\
\hline & dechlor. & 100 & 100 & \\
\hline & Oct 1988 & 100 & 100 & $0.00-0.00$ \\
\hline & Jul 1989 & 100 & 100 & $0.00-0.09$ \\
\hline & Jan 1990 & 100 & $<100$ & $0.00-0.00$ \\
\hline & May 1990 & 100 & 100 & $0.00-0.05$ \\
\hline \multirow[t]{10}{*}{190} & Jul 1988 & 25 & 25 & $0.00-0.99$ \\
\hline & Aug 1988 & $<100$ & $<100$ & $0.00-0.87$ \\
\hline & dechlor. & 100 & 100 & \\
\hline & Oct 1988 & $<100$ & $<100$ & $0.00-0.96$ \\
\hline & dechlor. & $<100$ & $<100$ & \\
\hline & Jul 1989 & 100 & $<100$ & $0.20-0.30$ \\
\hline & dechlor. & NT & 100 & \\
\hline & Jan 1990 & 100 & $<100$ & $0.00-0.26$ \\
\hline & dechlor. & 100 & 100 & \\
\hline & May 1990 & 100 & 100 & $0.00-0.06$ \\
\hline
\end{tabular}

aNo-observed-effect concentration designates the highest concentration of the effluent tested causing no significant $(p>0.05$ ) reduction in survival or growth of fathead minnow larvae, or survival or reproduction of Ceriodaphnia.

${ }^{b} \mathrm{NT}=$ not tested. 
Table 3.7. Mean (range in parentheses) concentration (in milligrams per liter) of selected parameters in Storm Drain 170 efiivent obtained by the Oak Ridge K-25 Site Process Support Department taken in conjunction with some of the toxicity tests

\begin{tabular}{|c|c|c|c|c|c|c|}
\hline Analysis & $\begin{array}{l}\text { Jul } 1988 \\
(n=3)^{a}\end{array}$ & $\begin{array}{l}\text { Aug } 1988 \\
(n=6)\end{array}$ & $\begin{array}{l}\text { Oct } 1988 \\
(n=1)\end{array}$ & $\begin{array}{c}\text { Dec } 1988 \\
(n=2)\end{array}$ & $\begin{array}{c}\text { Feb } 1989 \\
(n=7)\end{array}$ & $\begin{array}{c}\text { Jul } 1989 \\
(n=7)\end{array}$ \\
\hline Aluminum & $\begin{array}{c}0.07 \\
(0.05-0.1)\end{array}$ & $\begin{array}{c}0.14 \\
(0.02-0.51)\end{array}$ & 0.065 & $\begin{array}{c}0.24 \\
(0.23-0.25)\end{array}$ & $\begin{array}{c}0.50 \\
\left(B D^{\circ}-0.56\right)\end{array}$ & $\begin{array}{c}0.18 \\
(0.06-0.55)\end{array}$ \\
\hline Barium & $\begin{array}{c}0.028 \\
(0.027-0.029)\end{array}$ & $\begin{array}{c}0.024 \\
(0.02-0.027)\end{array}$ & 0.031 & $\begin{array}{c}0.026 \\
(0.026-0.026)\end{array}$ & $\begin{array}{c}0.041 \\
(B D-0.13)\end{array}$ & $\begin{array}{c}0.036 \\
(0.031-0.045)\end{array}$ \\
\hline Calcium & $\begin{array}{c}34 \\
(33-36)\end{array}$ & $\begin{array}{c}30 \\
(14-37)\end{array}$ & 33 & $\begin{array}{c}41 \\
(40-43)\end{array}$ & $\begin{array}{c}40 \\
(30-51)\end{array}$ & $\begin{array}{c}54 \\
(32-120)\end{array}$ \\
\hline Chloride & $\begin{array}{c}44 \\
(20-86)\end{array}$ & $\begin{array}{c}20 \\
(B D-28)\end{array}$ & 13 & $\begin{array}{c}29 \\
(26-32)\end{array}$ & $\begin{array}{c}18 \\
(15-27)\end{array}$ & $\begin{array}{c}48 \\
(2-154)\end{array}$ \\
\hline Chromium & $\begin{array}{c}0.16 \\
(B D-0.43)\end{array}$ & $\begin{array}{c}0.012 \\
(B D-0.02)\end{array}$ & 0.02 & $\begin{array}{c}0.020 \\
(0.015-0.024)\end{array}$ & BD & $\begin{array}{c}0.02 \\
(B D-0.03)\end{array}$ \\
\hline Copper & $\begin{array}{c}0.01 \\
(0.01-0.01)\end{array}$ & $\begin{array}{c}0.02 \\
(B D-0.06)\end{array}$ & 0.02 & $\begin{array}{c}0.012 \\
(B D-0.014)\end{array}$ & BD & $\begin{array}{c}0.04 \\
(B D-0.21)\end{array}$ \\
\hline Dissolved solids & $\begin{array}{c}284 \\
(212-396)\end{array}$ & $\begin{array}{c}213 \\
(96-252)\end{array}$ & 174 & $\begin{array}{c}285 \\
(280-290)\end{array}$ & $\begin{array}{c}205 \\
(164-274)\end{array}$ & $\begin{array}{c}339 \\
(164-722)\end{array}$ \\
\hline Fluoride & $\mathrm{BD}$ & $\begin{array}{c}0.12 \\
(\mathrm{BD}-0.2)\end{array}$ & 0.10 & $\begin{array}{c}0.25 \\
(0.2-0.3)\end{array}$ & BD & BD \\
\hline Iron & $\begin{array}{c}0.6 \\
(B D-1.5)\end{array}$ & $\begin{array}{c}0.2 \\
(0.01-0.44)\end{array}$ & 0.1 & $\begin{array}{c}0.4 \\
(0.40-0.41)\end{array}$ & BD & $\begin{array}{c}0.4 \\
(0.11-0.37)\end{array}$ \\
\hline Magnesium & $\begin{array}{c}9.4 \\
(8.9-10)\end{array}$ & $\begin{array}{c}7.5 \\
(2.2-8.9)\end{array}$ & 8.3 & $\begin{array}{c}10.2 \\
(9.3-11)\end{array}$ & $\begin{array}{c}8.9 \\
(5.9-12)\end{array}$ & $\begin{array}{c}10.8 \\
(9.1-14)\end{array}$ \\
\hline Manganese & $\begin{array}{c}0.02 \\
(\mathrm{BD}-0.04)\end{array}$ & $\begin{array}{c}0.02 \\
(0.02-0.03)\end{array}$ & 0.02 & $\begin{array}{c}0.04 \\
(0.03-0.05)\end{array}$ & $\begin{array}{c}0.04 \\
(B D-0.09)\end{array}$ & $\begin{array}{c}0.08 \\
(0.04-0.16)\end{array}$ \\
\hline Nickel & $\begin{array}{c}0.09 \\
(\mathrm{BD}-0.24)\end{array}$ & $\begin{array}{c}0.02 \\
(\mathrm{BD}-0.03)\end{array}$ & 0.01 & $\begin{array}{c}0.02 \\
(0.01-0.02)\end{array}$ & BD & $\begin{array}{c}0.03 \\
(B D-0.086)\end{array}$ \\
\hline
\end{tabular}


Table 3.7 (continued)

\begin{tabular}{|c|c|c|c|c|c|c|}
\hline Analysis & $\begin{array}{l}\text { Jul } 1988 \\
(n=3)\end{array}$ & $\begin{array}{c}\text { Aug } 1988 \\
(n=6)\end{array}$ & $\begin{array}{l}\text { Oct } 1988 \\
(n=1)\end{array}$ & $\begin{array}{c}\text { Dec } 1988 \\
(n=2)\end{array}$ & $\begin{array}{l}\text { Feb } 1989 \\
(n=7)\end{array}$ & $\begin{array}{c}\text { Jul } 1989 \\
(n=7)\end{array}$ \\
\hline Phosphorus & $\begin{array}{c}0.90 \\
(0.47-1.3)\end{array}$ & $\begin{array}{c}1.25 \\
\text { (BD-1.6) }\end{array}$ & 0.3 & $\begin{array}{c}1.22 \\
(0.65-1.8)\end{array}$ & $\mathbf{N M}^{c}$ & NM \\
\hline Potassium & $\begin{array}{c}3.5 \\
(3.3-3.7)\end{array}$ & $\begin{array}{c}5.6 \\
(3.3-6.4)\end{array}$ & NM & NM & $\begin{array}{c}37.7 \\
(19-54)\end{array}$ & $\begin{array}{c}9.3 \\
(1.5-13)\end{array}$ \\
\hline Silicon & $\begin{array}{c}1.2 \\
(0.8-1.6)\end{array}$ & $\begin{array}{c}2.4 \\
(2.2-2.5)\end{array}$ & 1.9 & $\begin{array}{c}2.1 \\
(1.9-2.3)\end{array}$ & $\begin{array}{c}0.8 \\
\text { (BD-1.3) }\end{array}$ & $\begin{array}{c}3.5 \\
(2.9-4.8)\end{array}$ \\
\hline Sodium & $\begin{array}{c}17 \\
(15-20)\end{array}$ & $\begin{array}{c}25 \\
(6-31)\end{array}$ & 12 & $\begin{array}{c}35 \\
(32-37)\end{array}$ & $\begin{array}{c}23 \\
(15-31)\end{array}$ & $\begin{array}{c}33 \\
(2-85)\end{array}$ \\
\hline Sulfate & $\begin{array}{c}90 \\
(47-169)\end{array}$ & $\begin{array}{c}52 \\
(16-64)\end{array}$ & 43 & $\begin{array}{c}81 \\
(79-82)\end{array}$ & $\begin{array}{c}46 \\
(34-72)\end{array}$ & $\begin{array}{c}100 \\
(12-235)\end{array}$ \\
\hline Suspended solids & $\mathrm{BD}$ & $\begin{array}{c}4.2 \\
\text { (BD-9) }\end{array}$ & 2.0 & $\begin{array}{c}4.5 \\
(3-6)\end{array}$ & BD & $\begin{array}{c}5.0 \\
(\mathrm{BD}-15)\end{array}$ \\
\hline Zinc & $\begin{array}{c}0.02 \\
(\mathrm{BD}-0.03)\end{array}$ & $\begin{array}{c}0.10 \\
(0.03-0.16)\end{array}$ & 0.09 & $\begin{array}{c}0.08 \\
(0.06-0.1)\end{array}$ & $\begin{array}{c}0.08 \\
(\mathrm{BD}-0.15)\end{array}$ & $\begin{array}{c}0.03 \\
(0.01-0.06)\end{array}$ \\
\hline
\end{tabular}

$a_{n}=$ sample size.

${ }^{b} \mathrm{BD}=$ below detection.

${ }^{\mathrm{NM}}=$ not measured.

Sources: McGaha, M. A, 1989a, Toxicity Monitoring at ORGDP July-September 1988, Report No. K/QT-288, Oak Ridge Gaseous Diffusion Plant, Oak Ridge, Tennessee; McGaha, M. A., 1989b, Toxicity Monitoring at ORGDP October-December 1988, Report No. K/QT-0311, Oak Ridge Gaseous Diffusion Plant, Oak Ridge, Tennessee; McGaha, M. A., 1989c, Toxicity Monitoring at ORGDP January-March 1989, Report No. K/QT-312, Oak Ridge Gaseous Diffusion Plant, Oak Ridge, Tennessee; McGaha, M. A., 1989d, Toxicity Monitoring at ORGDP April-June 1989, Report No. K/QT-313,

Oak Ridge Gaseous Diffusion Plant, Oak Ridge, Tennessee; and Shoemaker, J. L., et al., 1990, Toxicity Monitoring at ORGDP October-December 1989, Report No. K/QT-0373, Oak Ridge Gaseous Diffusion Plant, Oak Ridge, Tennessee. 
Table 3.8. Mean (range in parentheses) concentrations (in milligrams per liter) of selectod parameters in Storm Drain 180 effluent obtained by the Oak Ridge K-25 Site Process Support Department taken in conjunction with some of the toricity tests

\begin{tabular}{|c|c|c|c|c|}
\hline Analysis & $\begin{array}{l}\text { Jul } 1988 \\
(n=3)^{a}\end{array}$ & $\begin{array}{l}\text { Aug } 1988 \\
(n=6)\end{array}$ & $\begin{array}{l}\text { Oct } 1988 \\
(n=7)\end{array}$ & $\begin{array}{c}\text { Jul } 1989 \\
(n=7)\end{array}$ \\
\hline Aluminum & 0.07 & 3.90 & 1.70 & 0.06 \\
\hline Barium & $\begin{array}{c}0.044 \\
(0.042-0.045)\end{array}$ & $\begin{array}{c}0.068 \\
(0.047-0.17)\end{array}$ & $\begin{array}{c}0.096 \\
(0.06-0.12)\end{array}$ & $\begin{array}{c}0.057 \\
(0.047-0.068)\end{array}$ \\
\hline Calcium & $\begin{array}{c}39 \\
(38-41)\end{array}$ & $\begin{array}{c}36 \\
(20-41)\end{array}$ & $\begin{array}{c}56 \\
(30-70)\end{array}$ & $\begin{array}{c}73 \\
(43-160)\end{array}$ \\
\hline Chloride & $\begin{array}{c}20.7 \\
(19-23)\end{array}$ & $\begin{array}{c}15.3 \\
(B D-26)\end{array}$ & $\begin{array}{c}31.1 \\
(B D-44)\end{array}$ & $\begin{array}{c}64.0 \\
(26-166)\end{array}$ \\
\hline Chromium & $\begin{array}{c}0.15 \\
\text { (BD-0.37) }\end{array}$ & $\begin{array}{c}0.01 \\
(\mathrm{BD}-0.02)\end{array}$ & $\begin{array}{c}0.01 \\
(\mathrm{BD}-0.02)\end{array}$ & $\mathrm{BD}$ \\
\hline $\begin{array}{l}\text { Dissolved } \\
\text { solids }\end{array}$ & $\begin{array}{c}238 \\
(222-254)\end{array}$ & $\begin{array}{c}198 \\
(150-252)\end{array}$ & $\begin{array}{c}263 \\
(134-318)\end{array}$ & $\begin{array}{c}604 \\
(230-1788)\end{array}$ \\
\hline Fluoride & $\begin{array}{c}0.10 \\
(0.1-0.1)\end{array}$ & $\begin{array}{c}0.13 \\
(\mathrm{BD}-0.2)\end{array}$ & $\begin{array}{c}0.34 \\
(\mathrm{BD}-0.7)\end{array}$ & $\begin{array}{c}0.53 \\
(0.1-3.0)\end{array}$ \\
\hline Iron & $\begin{array}{c}0.4 \\
(\mathrm{BD}-1.2)\end{array}$ & $\begin{array}{c}3.6 \\
(0.01-21)\end{array}$ & $\begin{array}{c}1.9 \\
(\mathrm{BD}-11)\end{array}$ & $\begin{array}{c}0.10 \\
(0.05-0.17)\end{array}$ \\
\hline Magnesium & $\begin{array}{c}10.0 \\
(9.9-10)\end{array}$ & $\begin{array}{c}10.0 \\
(5.9-11)\end{array}$ & $\begin{array}{c}11.9 \\
(5.5-15)\end{array}$ & $\begin{array}{c}13.1 \\
(10-21)\end{array}$ \\
\hline Manganese & $\begin{array}{c}0.05 \\
(0.04-0.07)\end{array}$ & $\begin{array}{c}0.10 \\
(0.06-0.23)\end{array}$ & $\begin{array}{c}0.26 \\
(0.21-0.37)\end{array}$ & $\begin{array}{c}0.38 \\
(0.16-0.55)\end{array}$ \\
\hline Nickel & $\begin{array}{c}0.08 \\
(B D-0.19)\end{array}$ & $\mathrm{BD}$ & $\begin{array}{c}0.01 \\
(\mathrm{BD}-0.02)\end{array}$ & $\begin{array}{c}0.02 \\
(\mathrm{BD}-0.04)\end{array}$ \\
\hline Potassium & $\begin{array}{c}3.0 \\
(2.6-3.7)\end{array}$ & $\begin{array}{c}7.1 \\
(2.5-7.5)\end{array}$ & $\mathrm{NM}^{c}$ & $\begin{array}{c}12.4 \\
(1.9-58)\end{array}$ \\
\hline Silicon & $\begin{array}{c}1.3 \\
(0.1-1.6)\end{array}$ & $\begin{array}{c}7.6 \\
(2-35)\end{array}$ & $\begin{array}{c}4.6 \\
(2.2-15)\end{array}$ & $\begin{array}{c}3.2 \\
(2.8-4.6)\end{array}$ \\
\hline Sodium & $\begin{array}{c}8.6 \\
(8.1-9.3)\end{array}$ & $\begin{array}{c}8.1 \\
(1.8-10)\end{array}$ & $\begin{array}{c}13.5 \\
(5.8-18)\end{array}$ & $\begin{array}{c}73.2 \\
(7.9-340)\end{array}$ \\
\hline Sulfate & $\begin{array}{c}38.0 \\
(38-38)\end{array}$ & $\begin{array}{c}32.5 \\
(17-36)\end{array}$ & $\begin{array}{c}40.0 \\
(19-47)\end{array}$ & $\begin{array}{c}293.3 \\
(31-1090)\end{array}$ \\
\hline
\end{tabular}


Table 3.8 (continued)

\begin{tabular}{|c|c|c|c|c|}
\hline Analysis & $\begin{array}{l}\text { Jul } 1988 \\
(n=3)^{a}\end{array}$ & $\begin{array}{c}\text { Aug } 1988 \\
(n=6)\end{array}$ & $\begin{array}{l}\text { Oct } 1988 \\
(n=7)\end{array}$ & $\begin{array}{c}\text { Jul } 1989 \\
(n=7)\end{array}$ \\
\hline Suspended & BD & 83.5 & 30.9 & 2.9 \\
\hline Zinc & $\begin{array}{c}0.02 \\
\text { (BD-0.03) }\end{array}$ & $\begin{array}{c}0.10 \\
(0.06-0.13)\end{array}$ & $\begin{array}{c}0.06 \\
(0.03-0.08)\end{array}$ & $\begin{array}{c}0.02 \\
(0.01-0.03)\end{array}$ \\
\hline 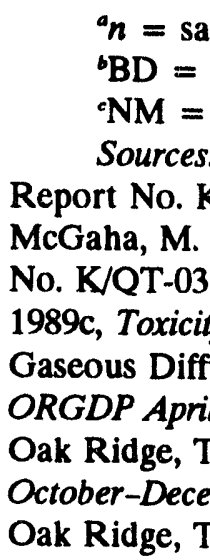 & $\begin{array}{l}\text { size. } \\
\text { detection. } \\
\text { measured. } \\
\text { Gaha, M. A } \\
288 \text {, Oak R } \\
989 \mathrm{~b} \text {, Toxicit } \\
\text { ak Ridge G } \\
\text { nitoring at O } \\
\text { Plant, Oak } \\
\text { e } 1989 \text {, Repc } \\
\text { ssee; and Sh } \\
1989 \text {, Repo } \\
\text { ssee }\end{array}$ & $\begin{array}{l}\text { Toxicity Mo } \\
\text { eous Diffusi } \\
\text { oring at ORG } \\
\text { jiffusion Pla } \\
\text { anuary-Marc } \\
\text { Tennessee; M } \\
\text { UQT-313, O } \\
\text { J. L., et al. } \\
\text { /QT-0373, O }\end{array}$ & $\begin{array}{l}\text { t ORGDP Ju } \\
\text { Oak Ridge, } \\
\text { ber-Decembe } \\
\text { Ridge, Tenne } \\
\text { Report No. I } \\
\text { M. A., 1989d } \\
\text { Gaseous Di } \\
\text { Toxicity Moni } \\
\text { Gaseous D }\end{array}$ & $\begin{array}{l}\text { nber 1988, } \\
\text { ee; } \\
\text { Report } \\
\text { Gaha, M. A., } \\
\text { 2, Oak Ridge } \\
\text { Monitoring at } \\
\text { Plant, } \\
\text { ORGDP } \\
\text { Plant, }\end{array}$ \\
\hline
\end{tabular}

Effluent from SD 190 typically contained the lowest concentrations of the parameters measured.

\subsubsection{Discussion}

\subsubsection{K-1407-B Pond}

During the two tests completed in 1988, the effluent from the K-1407-B pond was not toxic to fathead minnows or Ceriodaphnia. These were the last two tests completed before closure of the pond began.

\subsubsection{K-1407-E/F Pond}

Effluent from $\mathrm{K}-1407-\mathrm{E} / \mathrm{F}$ pond was never toxic to fathead minnows but was nearly always toxic to Ceriodaphnia. The mean effluent NOEC for Ceriodaphnia was $52.4 \%$ for those tests that were conclusive (range $=6$ to $100 \%$ ). The toxicity of the effluent appeared to be linked to those constituents that cause high hardness and conductivity levels (e.g., calcium and sulfate). For example, the maximum calcium concentration during the February 1989 test was $189 \mathrm{mg} / \mathrm{L}$. Assuming the $\mathrm{Ca}$ is balanced with $\mathrm{SO}_{4}$, the concentration of $\mathrm{CaSO}_{4}$ would equal $643 \mathrm{mg} / \mathrm{L}$. In a 7-d toxicity test with reagent-grade $\mathrm{CaSO}_{4}$, $615 \mathrm{mg}$ of $\mathrm{CaSO}_{4}$ per $\mathrm{L}$ reduced fecundity of Ceriodaphnia by $62 \%$ (L. A. Kszos, Environmental Sciences Division, and M. L. Holtz, University of Kentucky, unpublished data). The measured NOEC $(50 \%)$ for the February 1989 test with $\mathrm{K}-1407-\mathrm{E} / \mathrm{F}$ pond effluent could therefore be accounted for by the concentration of $\mathrm{CaSO}_{4}$ in the effluent. Although chemical analyses are not yet published for the tests 
Table 3.9. Mean (range in parentheses) concentrations (in milligrams per liter) of selected parameters in Storm Drain 190 effuent obtained by the Oak Ridge K-25 Site Process Support Department taken in conjunction with some of the toxicity tests

\begin{tabular}{|c|c|c|c|c|}
\hline Analysis & $\begin{array}{l}\text { Jul } 1988 \\
(n=4)^{a}\end{array}$ & $\begin{array}{c}\text { Aug } 1988 \\
(n=3)\end{array}$ & $\begin{array}{c}\text { Oct } 1988 \\
(n=7)\end{array}$ & $\begin{array}{c}\text { Jul } 1989 \\
(n=7)\end{array}$ \\
\hline Aluminum & 0.71 & 0.35 & 0.19 & 0.07 \\
\hline Barium & $\begin{array}{c}0.04 \\
(0.03-0.05)\end{array}$ & $\begin{array}{c}0.03 \\
(0.01-0.04)\end{array}$ & $\begin{array}{c}0.03 \\
(0.02-0.05)\end{array}$ & $\begin{array}{c}0.04 \\
(0.04-0.04)\end{array}$ \\
\hline Calcium & $\begin{array}{c}36.3 \\
(19-51)\end{array}$ & $\begin{array}{c}28.3 \\
(11-40)\end{array}$ & $\begin{array}{c}31.9 \\
(17-40)\end{array}$ & $\begin{array}{c}39.0 \\
(37-40)\end{array}$ \\
\hline Chloride & $\begin{array}{c}16.8 \\
(11-26)\end{array}$ & $\begin{array}{c}9.7 \\
\left(\mathrm{BD}^{b}-14\right)\end{array}$ & $\begin{array}{c}12.3 \\
(\mathrm{BD}-20)\end{array}$ & $\begin{array}{c}16.9 \\
(14-21)\end{array}$ \\
\hline Chromium & $\begin{array}{c}0.41 \\
(\mathrm{BD}-0.62)\end{array}$ & $\begin{array}{c}0.01 \\
\text { (BD-0.01) }\end{array}$ & BD & $\mathrm{BD}$ \\
\hline Copper & $\begin{array}{c}0.02 \\
(0.01-0.04)\end{array}$ & $\begin{array}{c}0.015 \\
\text { (BD-0.025) }\end{array}$ & $\begin{array}{c}0.013 \\
(\mathrm{BD}-0.029)\end{array}$ & $\begin{array}{c}0.008 \\
(\mathrm{BD}-0.02)\end{array}$ \\
\hline $\begin{array}{l}\text { Dissolved } \\
\text { solids }\end{array}$ & $\begin{array}{c}208 \\
(70-286)\end{array}$ & $\begin{array}{c}148 \\
(94-184)\end{array}$ & $\begin{array}{c}158 \\
(68-196)\end{array}$ & $\begin{array}{c}190 \\
(98-212)\end{array}$ \\
\hline Fluoride & $\begin{array}{c}0.10 \\
(0.01-0.10)\end{array}$ & $\begin{array}{c}0.10 \\
\text { (BD-0.10) }\end{array}$ & $\begin{array}{c}0.20 \\
\text { (BD-0.30) }\end{array}$ & $\begin{array}{c}0.10 \\
(0.10-0.10)\end{array}$ \\
\hline Iron & $\begin{array}{c}2.0 \\
(1.8-2.3)\end{array}$ & $\begin{array}{c}0.4 \\
(0.28-0.49)\end{array}$ & $\begin{array}{c}0.4 \\
(0.12-1.40)\end{array}$ & $\begin{array}{c}0.20 \\
(0.13-0.44)\end{array}$ \\
\hline Magnesium & $\begin{array}{c}8.8 \\
(4.4-11)\end{array}$ & $\begin{array}{c}7.6 \\
(1.9-11)\end{array}$ & $\begin{array}{c}8.9 \\
(3.6-12)\end{array}$ & $\begin{array}{c}9.9 \\
(9.8-10)\end{array}$ \\
\hline Manganese & $\begin{array}{c}0.08 \\
(0.06-0.10)\end{array}$ & $\begin{array}{c}0.04 \\
(0.03-0.05)\end{array}$ & $\begin{array}{c}0.06 \\
(0.03-0.09)\end{array}$ & $\begin{array}{c}0.07 \\
(0.06-0.09)\end{array}$ \\
\hline Nickel & $\begin{array}{c}0.21 \\
(\mathrm{BD}-0.33)\end{array}$ & $\begin{array}{c}0.01 \\
(\mathrm{BD}-0.02)\end{array}$ & $\begin{array}{c}0.01 \\
(\mathrm{BD}-0.03)\end{array}$ & $\mathrm{BD}$ \\
\hline Potassium & $\begin{array}{c}2.8 \\
(2.1-3.8)\end{array}$ & $\begin{array}{c}1.4 \\
\text { (BD-2.2) }\end{array}$ & $\mathrm{NM}^{c}$ & $\begin{array}{c}1.8 \\
(1.0-2.3)\end{array}$ \\
\hline Silicon & $\begin{array}{c}1.8 \\
(1.6-2.1)\end{array}$ & $\begin{array}{c}1.9 \\
(1.5-2.2)\end{array}$ & $\begin{array}{c}1.6 \\
(1.0-2.3)\end{array}$ & $\begin{array}{c}2.7 \\
(2.6-3.0)\end{array}$ \\
\hline Sodium & $\begin{array}{c}6.2 \\
(0.9-9.3)\end{array}$ & $\begin{array}{c}5.1 \\
(1.7-7.1)\end{array}$ & $\begin{array}{c}5.7 \\
(2.5-7.6)\end{array}$ & $\begin{array}{c}5.9 \\
(2.6-6.5)\end{array}$ \\
\hline
\end{tabular}


Table 3.9 (continued)

\begin{tabular}{|c|c|c|c|c|}
\hline Analysis & $\begin{array}{l}\text { Jul } 1988 \\
(n=4)^{a}\end{array}$ & $\begin{array}{l}\text { Aug } 1988 \\
(n=3)\end{array}$ & $\begin{array}{l}\text { Oct } 1988 \\
(n=7)\end{array}$ & $\begin{array}{c}\text { Jul } 1989 \\
(n=7)\end{array}$ \\
\hline Sulfate & 34.8 & 25.3 & 29.6 & 31.6 \\
\hline $\begin{array}{l}\text { Suspended } \\
\text { solids }\end{array}$ & $\begin{array}{c}23.5 \\
\text { (BD-86) }\end{array}$ & $\begin{array}{c}11.0 \\
(7-16)\end{array}$ & $\begin{array}{c}5.4 \\
(\mathrm{BD}-15)\end{array}$ & $\begin{array}{c}2.1 \\
(\mathrm{BD}-6)\end{array}$ \\
\hline Zinc & $\begin{array}{c}0.10 \\
(0.05-0.25)\end{array}$ & $\begin{array}{c}0.10 \\
(0.09-0.12)\end{array}$ & $\begin{array}{c}0.10 \\
(0.06-0.14)\end{array}$ & $\begin{array}{c}0.05 \\
(0.04-0.07)\end{array}$ \\
\hline $\begin{array}{r}\quad \begin{array}{l}n \\
n=\mathrm{sa}\end{array} \\
\quad \mathrm{BD}= \\
\text { 'NM }= \\
\text { Sources } \\
\text { Report No. } \\
\text { McGaha, M. } \\
\text { No. K/QT-03 } \\
\text { 1989c, Toxici } \\
\text { Gaseous Dif } \\
\text { ORGDP Apr } \\
\text { Oak Ridge, } \\
\text { October-Dec } \\
\text { Oak Ridge, }\end{array}$ & $\begin{array}{l}\text { tion. } \\
\text { d. } \\
\text { M. A., 1989a } \\
\text { ak Ridge G } \\
\text { roxicity Moni } \\
\text { lge Gaseous } \\
\text { g at ORGDP } \\
\text { Oak Ridge, } \\
\text { Report No. } \\
\text { nd Shoemak } \\
\text { Report No. }\end{array}$ & $\begin{array}{l}\text { ity Monitorin } \\
\text { Diffusion Pla } \\
\text { it ORGDP O } \\
\text { on Plant, Oa } \\
\text { y-March } 198 \\
\text { ssee; McGah } \\
\text { 313, Oak Ri } \\
\text {, et al., } 1990 \\
\text { 373, Oak Ri }\end{array}$ & $\begin{array}{l}\text { DP July-Sept } \\
\text { Ridge, Tenne } \\
\text { ecember } 1988 \\
\text { Tennessee; } \\
\text { t No. K/QT-3 } \\
\text { 1989d, Toxic } \\
\text { ous Diffusion } \\
\text { Monitoring } \\
\text { ous Diffusior }\end{array}$ & $\begin{array}{l}1988, \\
\text { ort } \\
\text { ha, M. A., } \\
\text { Dak Ridge } \\
\text { onitoring at } \\
\text { tt, } \\
\text { ?GDP } \\
\text { t, }\end{array}$ \\
\hline
\end{tabular}

conducted during April and June 1990, the low NOECs for Ceriodaphnia are probably linked to high $\mathrm{Ca}$, as indicated by the high hardness of the effluents (741 and $814 \mathrm{mg} / \mathrm{L}$ respectively). Nickel may also contribute to the toxicity of the effluent. During the October 1989 test, the mean concentration of nickel was 0.32 $\mathrm{mg} / \mathrm{L} ; 0.05 \mathrm{mg} / \mathrm{L}$ in Bear Creek water is toxic to Ceriodaphnia (Kszos et al. 1992).

The toxicity and water quality of K-1407-E/F ponds and the closed K-1407-B pond were similar. Although the toxicity tests of the K-1407-B pond effluent were often inconclusive (adverse affect at the lowest concentration tested), the K-1407-B ponds and the K-1407-E/F pond effluent NOECs were $<100 \%$ for 8 of 12 Ceriodaphnia tests completed. Although effluent from the K-1407-E/F ponds was never toxic to fathead minnows, effluent NOEC from the K-1407-B pond was $<100 \%$ in three of ten tests. Water quality of effluents from the K-1407-E/F and $\mathrm{K}-1407-\mathrm{B}$ ponds were also similar: average values for the mean concentrations of alkalinity, hardness, and conductivity measured for all tests conducted were 69.6 vs $73.3 \mathrm{mg} / \mathrm{L}, 544$ vs $484 \mathrm{mg} / \mathrm{L}$, and 2122 vs $1829 \mu \mathrm{S} / \mathrm{cm}$ respectively.

The effect of the K-1407-E/F pond effluents on the aquatic biota in Mitchell Branch will obviously depend on the flow characteristics of the stream. During periods when flow in Mitchell Branch is low and the concentration of the effluent in the stream is $>50 \%$, the toxicity tests indicate that K-1407-E/F pond 
effluent may adversely affect the stream biota. Currently, however, the toxicity of the TRC discharged from SD 170 overwhelms the toxicity that might be caused in Mitchell Branch because of inputs of effluent from the K-1407-E/F ponds (see discussion of storm drains, Sect. 3.1.4.4). During periods when flow in Mitchell Branch is high, it is less likely that the pond effluent will adversely affect the stream biota.

\subsubsection{K-1407-J Basin}

Effluent from the K-1407-J basin was generally not toxic to fathead minnows; an NOEC of $<100 \%$ was obtained in only one test. Ceriodaphnia, on the other hand, were much more sensitive to this effluent, with a mean NOEC of $36.6 \%$ for those tests that were definitive (range: 1 to $100 \%$ ). The toxicity of the K-1407-J basin effluent is partly the result of the high concentrations of $\mathrm{Na}, \mathrm{Cl}$, and $\mathrm{SO}_{4}$ in the effluent. During the June 1989 test, for example, $1671 \mathrm{mg}$ of $\mathrm{Na}$ per liter in the effluent balanced ironically with $\mathrm{Cl}$ and $\mathrm{SO}_{4}$, yielding total concentrations of $\mathrm{NaCl}$ and $\mathrm{Na}_{2} \mathrm{SO}_{4}$ in the effluent of $\sim 3942 \mathrm{mg} / \mathrm{L}$ and $682 \mathrm{mg} / \mathrm{L}$ respectively. In Ceriodaphnia toxicity tests with reagentgrade $\mathrm{NaCl}$ and $\mathrm{Na}_{2} \mathrm{SO}_{4}, 1225 \mathrm{mg}$ of $\mathrm{NaCl}$ per liter was acutely toxic and $500 \mathrm{mg}$ of $\mathrm{NaSO}_{4}$ per liter reduced fecundity (L. A. Kszos and L. F. Wicker, Environmental Sciences Division, unpublished data). Thus, $3942 \mathrm{mg}$ of $\mathrm{NaCl}$ per liter in the K-1407-J basin effluent would be toxic to Ceriodaphnia at a concentration of $30 \%$; the measured lowest-observedeffect concentration (LOEC) during the June 1989 test was $25 \%($ NOEC $=12 \%)$. The toxicity of the effluent, however, may not always be accounted for by the presence of high $\mathrm{Na}, \mathrm{Cl}$, and/or $\mathrm{SO}_{4}$. For example, during the April 1989 test, $313 \mathrm{mg}$ of $\mathrm{NaCl}$ per liter and $424 \mathrm{mg}$ of $\mathrm{Na}_{2} \mathrm{SO}_{4}$ per liter were present (assuming the $\mathrm{Cl}$ and $\mathrm{SO}_{4}$ were completely balanced with $\mathrm{Na}$ ). Neither of these concentrations were found to be toxic in the tests with reagent-grade chemicals, yet the measured NOEC was $50 \%$. Thus, it appears that there are one or more unknown toxicant(s) present in the effluent besides $\mathrm{Na}, \mathrm{Cl}$, and $\mathrm{SO}_{4}$.

Because K-1407-J effluent is discharged to Poplar Creek, this wastewater does not affect the biota in Mitchell Branch. The receiving water concenkation of the effluent in Poplar Creek is $1 \%$ (B. A. Shoemaker, ORGDP Health, Safety and Environment Division, personal communication), thus, it is unlikely that the biota of Poplar Creek would be adversely affected.

\subsubsection{Storm drains}

High TRC is a major source of toxicity in SDs 170 and 190. The lowest maximum concentration of TRC in SDs 170 and 190 was $0.22 \mathrm{mg} / \mathrm{L}$ and $0.06 \mathrm{mg} / \mathrm{L}$ respectively. In general, TRC concentrations of about $0.20 \mathrm{mg} / \mathrm{L}$ are lethal to Ceriodaphnia (A. J. Stewart, Environmental Sciences Division, unpublished data). The EPA water quality criterion for $\mathrm{Cl}$ is even lower $(0.011 \mathrm{mg} / \mathrm{L}$; EPA 1986). Effluent from SD 170 was always toxic to Ceriodaphnia and in most cases, to fathead minnows. Dechlorinating the effluent from SD 170 did not always eliminate the toxicity to either species, indicating the presence of one or more toxicants other than chlorine in the effluent.

Effluent from SD 190 was toxic to Ceriodaphnia during all tests but one; the maximum concentration of TRC during the one test was $0.06 \mathrm{mg} / \mathrm{L}$. Dechlorination of the effluent always removed the toxicity to fathead minnows, but did not always eliminate toxicity to Ceriodaphnia. Ceriodaphnia were therefore more sensitive than fish to the toxicant(s) other than chlorine in the effluent. 
Effluent from SD 180 typically contained only low levels of TRC and was the least toxic of the storm drain effluents. In the two tests with elevated TRC, the effluent was toxic to both species. Effluent collected during one of the four tests without elevated TRC was toxic to Ceriodaphnia. (Metal concentrations in this effluent are not yet available.)

Chemical analyses of metals and other miscellaneous constituents in the effluent from the storm drains (Tables 3.7 through 3.9) provided little insight. Effluents that were dechlorinated but still toxic did not appear to have any constituents that were elevated relative to the dechlorinated effluent that was not toxic.

Effluents from the storm drains, particularly SDs 170 and 190, have a high potential to adversely impact aquatic life in Mitchell Branch. Measurements of TRC taken at the end-of-pipe (as reported above) and the toxicity tests indicate that the TRC in these effluents will adversely affect the biota. In addition, tests with dechlorinated effluents have shown that, on occasion, other constituents in the effluents may contribute to toxicity.

\subsection{AMBIENT TOXICITY}

\subsubsection{Introduction}

Ambient toxicity testing was incorporated into the Mitchell Branch BMAP to (1) evaluate area-source contributions to stream toxicity, (2) characterize patterns of toxicity in Mitchell Branch, (3) document changes in water quality attributable to changes in operations at the Oak Ridge K-25 Site, and (4) provide data to demonstrate that the effluent limitations established for the Oak Ridge K-25 Site protect and maintain the use of Mitchell Branch for growth and propagation of fish and aquatic life (Loar et al. 1992a). The sites chosen for testing were selected to bracket area- and point-source discharges into the stream and to correspond closely to those selected as instream monitoring study sites.

\subsection{Materials and Methods}

Ambient toxicity was evaluated by means of the fathead minnow (Pimephales promelas) larval survival and growth test and the Ceriodaphnia survival and reproduction test as described by Horning and Weber (1985). These tests are 7-d staticrenewal chronic tests, based on the survival and growth of the fathead minnow and survival and fecundity of the microcrustacean Ceriodaphnia.

The six sites evaluated were located at MIKs 1.43, 1.0, 0.71, 0.54, 0.45, and 0.12 (Fig. 2.1). MIK 1.43 was selected as a reference site because it is located upstream of the Oak Ridge K-25 Site operations and any know source of perturbation. Twenty-two tests were conducted on a bimonthly basis from January 1987 through July 1990 . Water sampling and water chemistry analyses were conducted as described in Sect. 3.1.2. Water collected from MIK 0.12 , however, was a daily $24-\mathrm{h}$ composite for the first four tests and a daily grab sample for the remaining tests. The switch to daily grab samples for this site was made when it was discovered that TRC could sometimes be detected at MIK 0.12 .

All data analyses were accomplished as described in Sect. 3.1.2, with the following exceptions. Differences in hardness, alkalinity, conductivity, fathead minnow growth, and Ceriodaphnia reproduction between sites were evaluated with an analysis of variance (ANOVA) by using the SAS general linear models (GLM) procedure (SAS 1985a, 1985b); significant differences were then separated with Tukey's studentized range test. The Tukey test was selected to test for differences because it accommodates unequal sample 
sizes, vhereas Dunnett's test does not. The AlNOVA proved to be inappropriate for distinguishing differences among sites and years for fathead minnow and Ceriodaphnia survival because the values at most sites had bimodal distributions (i.e., indicative of an all-or-none response). Unless otherwise noted, statements of significance are based on $p<0.05$.

\section{2 .3 Results}

\subsubsection{Water chemistry}

Measurements taken of three of the water quality parameters (conductivity, hardness, and alkalinity) are presented in Fig. 3.1. Six tests per year were completed ( $7 \mathrm{~d}$ of analyses per test) in 1987, 1988, and 1989; four tests were completed in 1990. Each year, conductivity and hardness increased with distance downstream. During 1987 and 1988, three reaches of the stream were distinctly different based on conductivity and hardness: (1) MIKs 1.43 and 1.0; (2) MIKs 1.0 and 0.71 ; and (3) MIKs $0.54,0.45$ and 0.12 . The increase in mean conductivity and hardness from MIKs 1.43 to 0.12 was $\sim 600 \mu \mathrm{S} / \mathrm{cm}$ and $140 \mathrm{mg} / \mathrm{L}$ respectively. The largest increase in hardness and conductivity occurred downstream of the K-1407-B pond discharge point between MIKs 0.71 and 0.54. In 1989 and 1990, three reaches of the stream were still distinct based on significant differences in conductivity: (1) MIK 1.43; (2) MIK 1.0; and (3) MIKs $0.7_{i}, 0.54,0.45$, and 0.12 . The sharp increase between MIKs 0.71 and 0.54 no longer existed. In 1989 and 1990 , downstream increases in conductivity were less dramatic, with mean conductivity from MIKs 1.43 to 0.12 increasing by only about $300 \mu \mathrm{S} / \mathrm{cm}$. This reduction was the result of a drop in conductivity at MIKs 0.54, 0.45, and 0.12 during 1989 and 1990; there was no significant change in the conductivity at MIK 0.71 from 1987 to 1990. In 1989 and 1990 , mean hardness showed a trend similar to that of conductivity; there was no significant difference in mean hardness from MIKs 0.71 to 0.45 in 1989 or from MIKs 0.71 to 0.12 in 1990. In 1990, the increase in hardness from MIKs 1.43 to 0.12 was $120 \mathrm{mg} / \mathrm{L}$.

Trends for alkalinity in Mitchell Branch were different than those for conductivity and hardness. In 1987 , alkalinity at MIK 1.0 was significantly higher than at MIKs $1.43,0.71,0.54$, and 0.45 ; there was no significant difference in alkalinity from MIKs 0.71 to 0.12 . During 1988, alkalinity also increased at MIK 1.10, but unlike the trend in 1987, alkalinity remained elevated at all downstream sites. In 1989 and 1990, there was a more gradual increase in alkalinity with distance downstream. In 1989, alkalinity at MIK 0.12 was significantly higher than that at all upstream sites, with no difference from MIKs 0.71 to 0.45 . In 1990 , there was no difference in alkalinity from MIKs 0.71 to 0.12 .

The $\mathrm{pH}$ in Mitchell Branch generally increased with distance downstream (Table 3.10). During 1988, water from MIKs 0.54, 0.45, and 0.12 had the largest range in values. During the remaining years, the range in values at each site was similar.

Cumulative frequency distributions of TRC for all sampling dates since 1987 at MIKs $0.12,0.45,0.54$, and 0.71 are shown in Fig. 3.2. The concentration of TRC in Mitchell Branch was typically highest at MIK 0.71 (below SD 170). At MIK $0.71, \sim 40 \%$ of the measured values were $>0.20 \mathrm{mg} / \mathrm{L}$ (the concentration that is usually lethal to Ceriodaphnia; A. J. Stewart, Environmental Sciences Division, unpublished data). The concentration of TRC diminished with distance downstream: $30 \%$ of the TRC values at MIK 0.54 were $>0.20 \mathrm{mg} / \mathrm{L}$, about $25 \%$ of the values at MIK 0.45 were $>0.20 \mathrm{mg} / \mathrm{L}$, 
ORNL-DWG 90M-16734
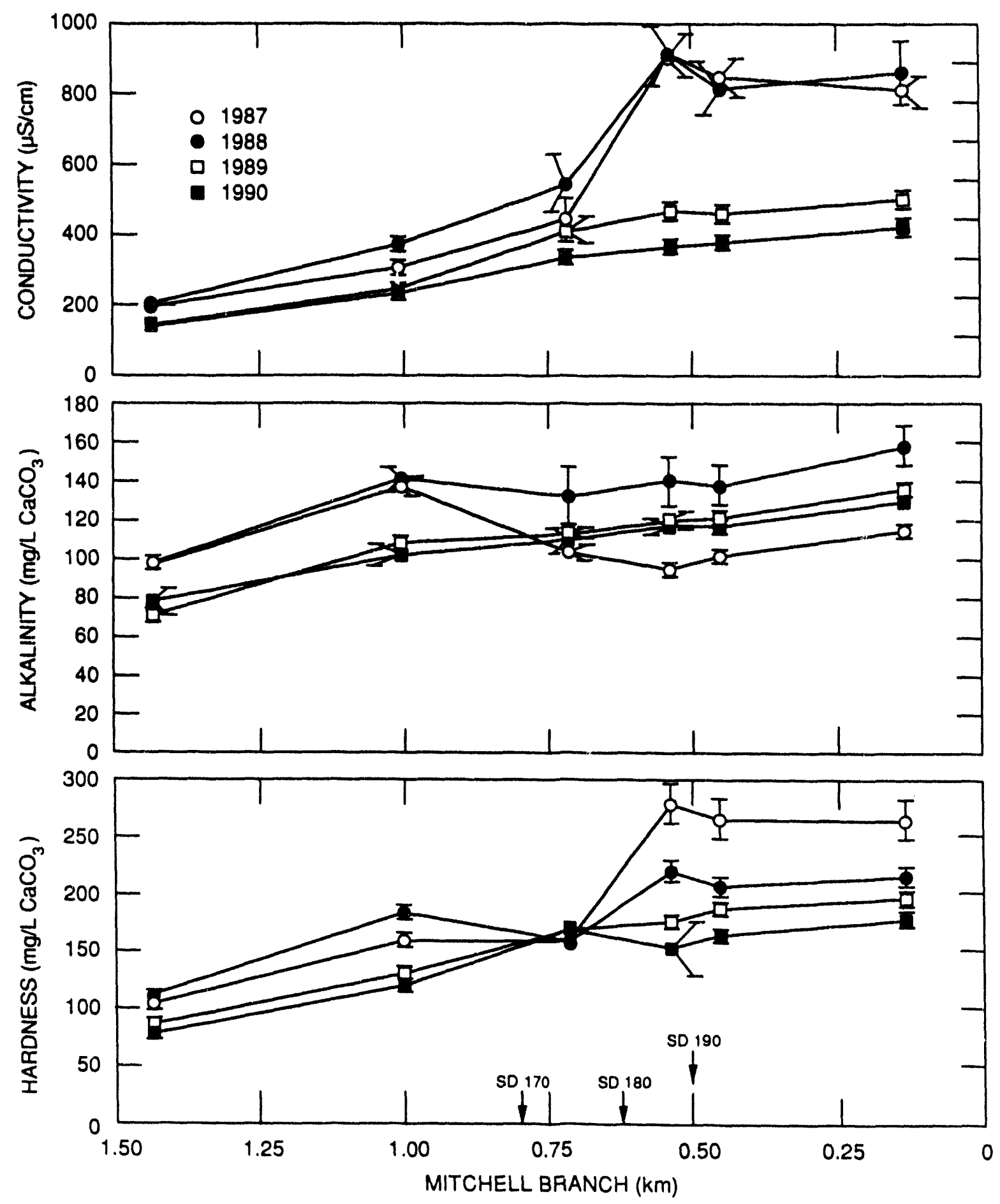

Fig. 3.1. Annual mean conductivity, alkalinity, and hardness for each site in Mitchell Branch, 1987-90. Vertical bars represent \pm 1 SE. $S D=$ storm drain. 
Table 3.10. Range in $\mathrm{pH}$ values at each site in Mitchell Branch for all tests within each year

\begin{tabular}{lcccc}
\hline & \multicolumn{5}{c}{ Range in pH values } \\
\cline { 2 - 5 } Site & 1987 & \multicolumn{5}{c}{1988} & 1989 & 1990 \\
\hline MIK 0.12 & $7.72-8.42$ & $7.06-8.90$ & $7.61-8.40$ & $7.74-8.18$ \\
MIK 0.45 & $7.74-8.21$ & $7.01-8.48$ & $7.54-8.12$ & $7.73-8.20$ \\
MIK 0.54 & $7.82-8.21$ & $7.29-8.37$ & $7.52-8.09$ & $7.61-8.21$ \\
MIK 0.71 & $7.79-8.34$ & $7.88-8.32$ & $7.48-8.14$ & $7.62-8.23$ \\
MIK 1.0 & $7.78-8.29$ & $7.73-8.20$ & $7.53-8.12$ & $7.49-8.14$ \\
MIK 1.43 & $7.52-8.18$ & $7.64-8.40$ & $7.30-8.11$ & $7.13-8.53$ \\
\hline
\end{tabular}

Note: MIK = Mitchell Branch kilometer.

ORNL-DWG 90M-16737

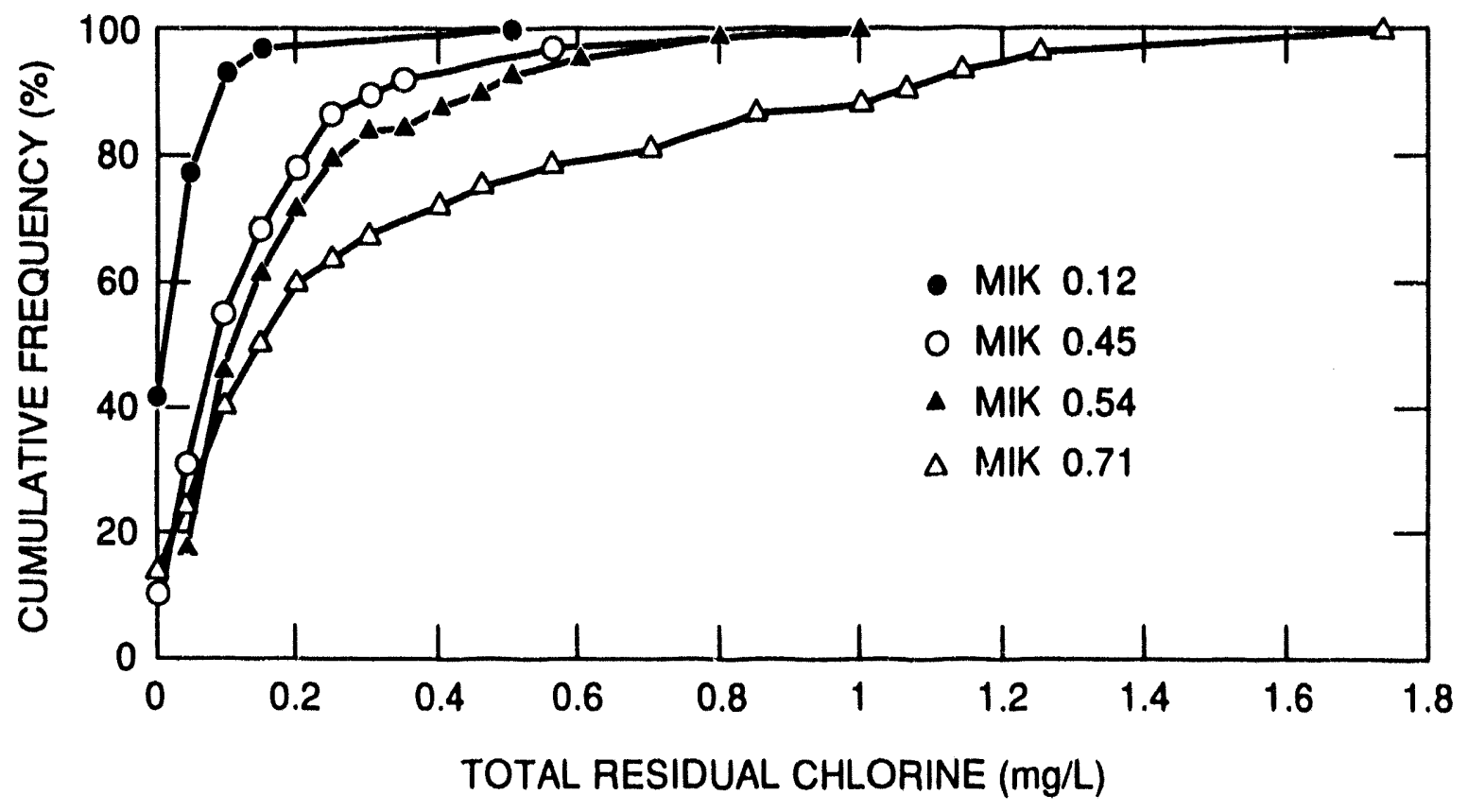

Fig. 3.2. Cumulative frequency (percentage) of total residual chlorine at 4 sites in Mitchell Branch for 22 tests, 1987-90.

and only about $5 \%$ of the values at MIK 0.12 were $>0.20 \mathrm{mg} / \mathrm{L}$. Cumulative frequency distributions of TRC for each year at MIKs $0.12,0.45,0.54$, and 0.71 are shown in Figs. 3.3 and 3.4. At all four sites, TRC concentrations were highest in 1988. During 1987, 1989, and 1990, TRC at MIK 0.12 was never higher than $0.20 \mathrm{mg} / \mathrm{L}$; however, in 1988 , TRC was $\geq 0.20 \mathrm{mg} / \mathrm{L}$ about $16 \%$ of the time. 

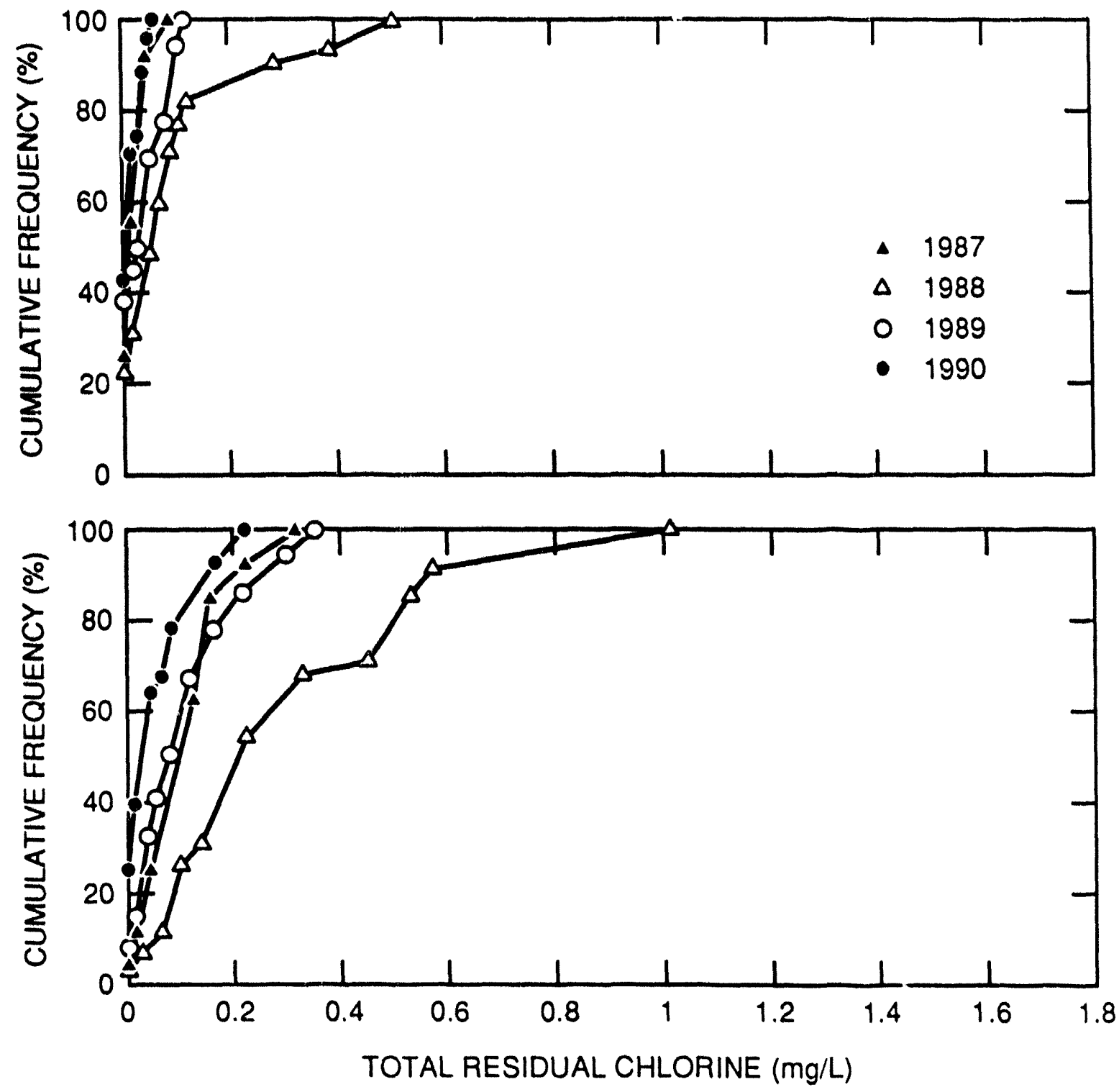

Fig. 3.3. Cumulative frequency (percentage) of total residual chlorine at Mitchell Branch kilometer (MIK) 0.12 (top) and MIK 0.45 (bottom), 1987-90.

During 1987, 1989, and 1990, TRC at MIK 0.45 was $>0.20 \mathrm{mg} / \mathrm{L} 10$ to $20 \%$ of the time; during 1988 , TRC was $\geq 0.20 \mathrm{mg} / \mathrm{L}$ about $60 \%$ of the time. TRC concentrations at MIK 0.54 and MIK 0.71 were more variable between years. In 1987, TRC at MIK 0.54 was $\geq 0.20 \mathrm{mg} / \mathrm{L}$ $10 \%$ of the time; during 1988 and 1990 , TRC was $\geq 0.20 \mathrm{mg} / \mathrm{L}$ about $30 \%$ of the time; and in 1990, TRC at MIK 0.54 was never $>20 \mathrm{mg} / \mathrm{L}$. In 1987, 1988, 1989 , and 1990, TRC at MIK 0.71 has been $>0.20 \mathrm{mg} / \mathrm{L}$ about $50,70,40$, and $20 \%$ of the time respectively.

Results of chemical analyses obtained concurrently with some of the toxicity tests by the Oak Ridge K-25 Site Process Support Department are summarized in 
ORNL.DWG 90M-16736
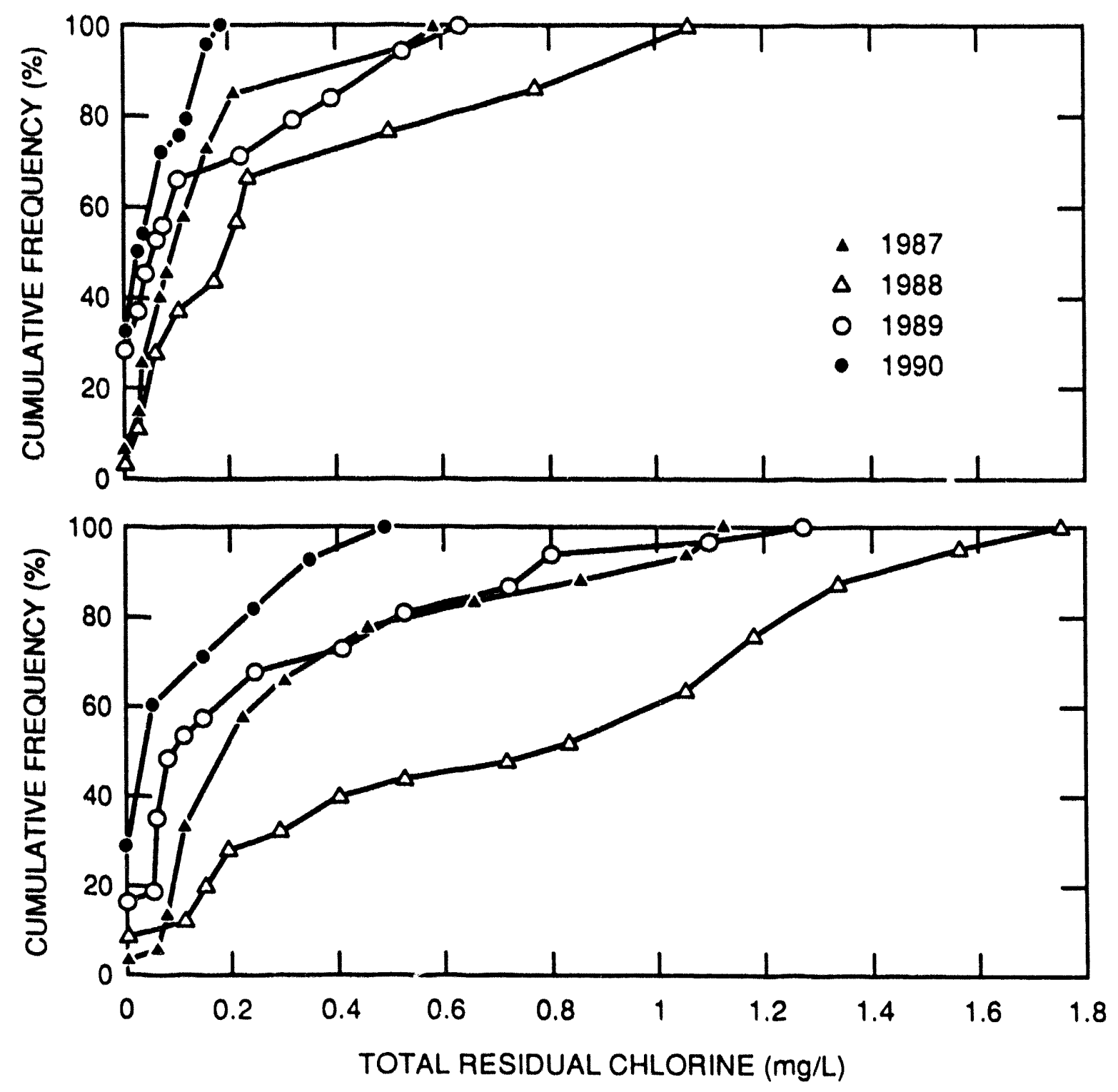

Fig. 3.4. Cumulative frequency (percentage) of total residual chlorine at Mitchell Branch kilometer (MIK) 0.54 (top) and MIK 0.71 (bottom), 1987-90.

Appendix B, Tables B.1 through B.9. Only those constituents that had concentrations consistently above detection levels or that were of toxicological concern were included; complete data sets are available elsewhere (McGaha 1989a, 1989b, 1989c, 1989d, 1989e; Shoemaker et al. 1990). The concentrations of none of the measured constituents at any of the sites were high enough to correlate with the toxicity observed. However, the concentration of nearly every constituent increased with distance downstream. Chemical data collected before and after the K-1407-B pond closed (October 1988) showed the influence that the pond had on water chemistry in Mitchell Branch. During the September 1988 test, before the pond 
closed, concentrations of chloride, dissolved solids, and sulfates were 2.5 to 4.2 times higher at MIK 0.54 (located below the K-1407-B outfall) than at MIK 0.71 [located above the K-1407-B outfall (Table B.2)]. In the November 1988 test, after the pond had closed, there were only minor differences $(<1.1$ times higher) between these two sites in the same parameters (Table B.3). Of the eight test dates shown, $\mathrm{A}$, dissolved solids, chloride, fluoride, $\mathrm{Mn}, \mathrm{Si}, \mathrm{Na}$, sulfate, and suspended solids were generally highest at all sites during the November 1988 test.

\subsubsection{Fathead minnow tests}

Mean survival and growth of fathead minnows for each site in each year are plotted in Fig. 3.5. Minnow survival in water from the reference site (MIK 1.43) was low (50 to $75 \%$ ) during each year
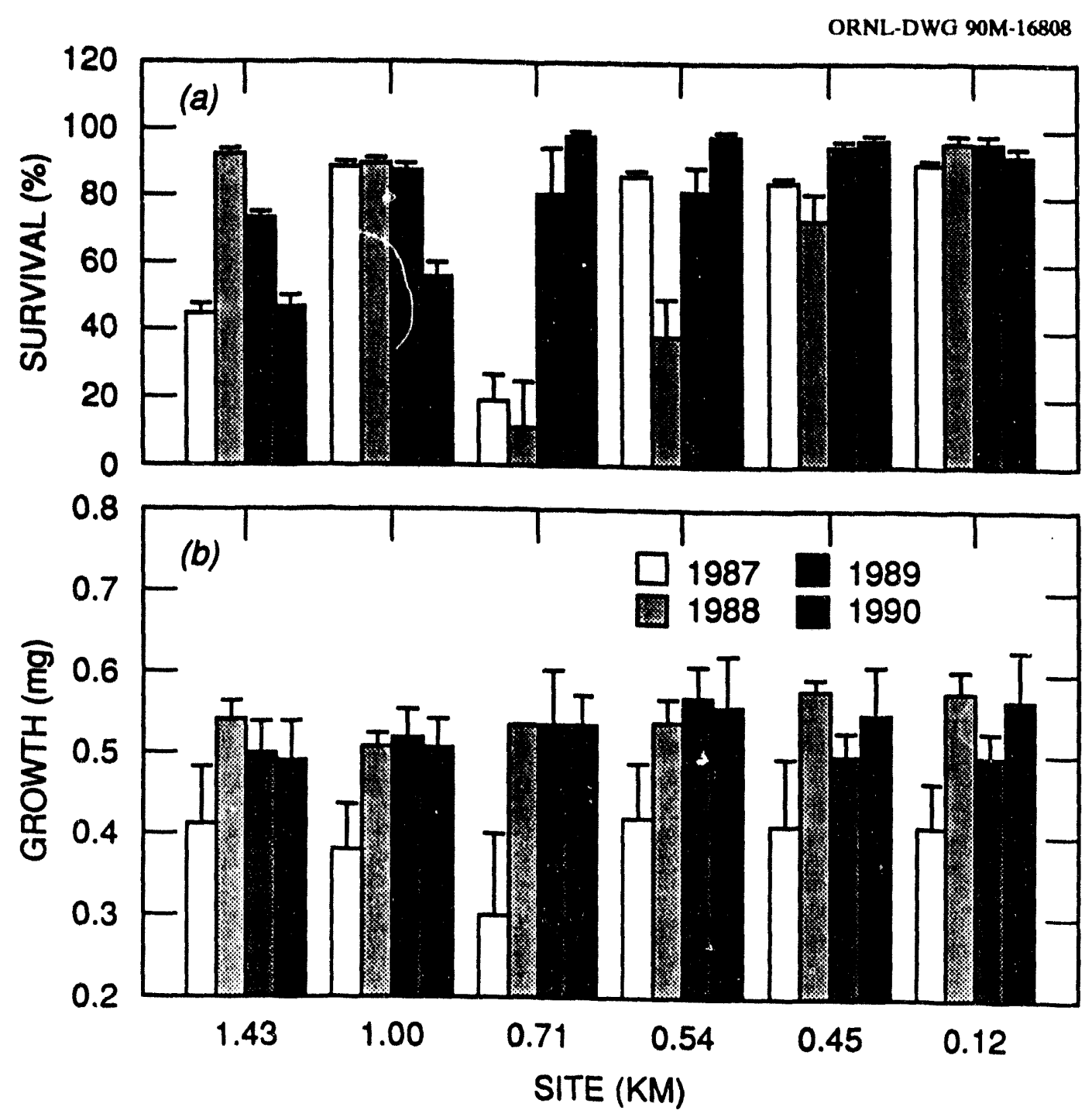

Fig. 3.5. Mean percentage survival (a) and growth of fathead minnows (b) for each site in Mitchsll Branch, 1987-90. Vertical bars represent \pm 1 SE. 
except for 1988. At MIK 1.0, mean survival was about $90 \%$ in each year except for 1990 , when mean survival was $55 \%$. Survival at MIK 0.71 was much lower than at all other sites in 1987 and all but MIK 0.54 in 1988. Mean survival of the minnows at MIK 0.54 dropped substantially from 1987 to 1988 but rebounded in 1989 and 1990, whereas survival at MIKs 0.45 and 0.12 remained relatively high in all 4 years. In 1989 and 1990, mean survival at MIKs 0.71, 0.54, 0.45, and 0.12 were similar. Also, survival at these four sites during the same 2-year period was greater than that at MIKs 1.43 and 1.0. Growth of the fish in water from all sites was significantly lower in 1987 compared with growth in 1988, 1989, and 1990 . Within eack year, however, there were no significant differences in growth among sites. The frequency distributions of survival at each site for each year (Figs. 3.6

ORNL-DWG 90M-16807A

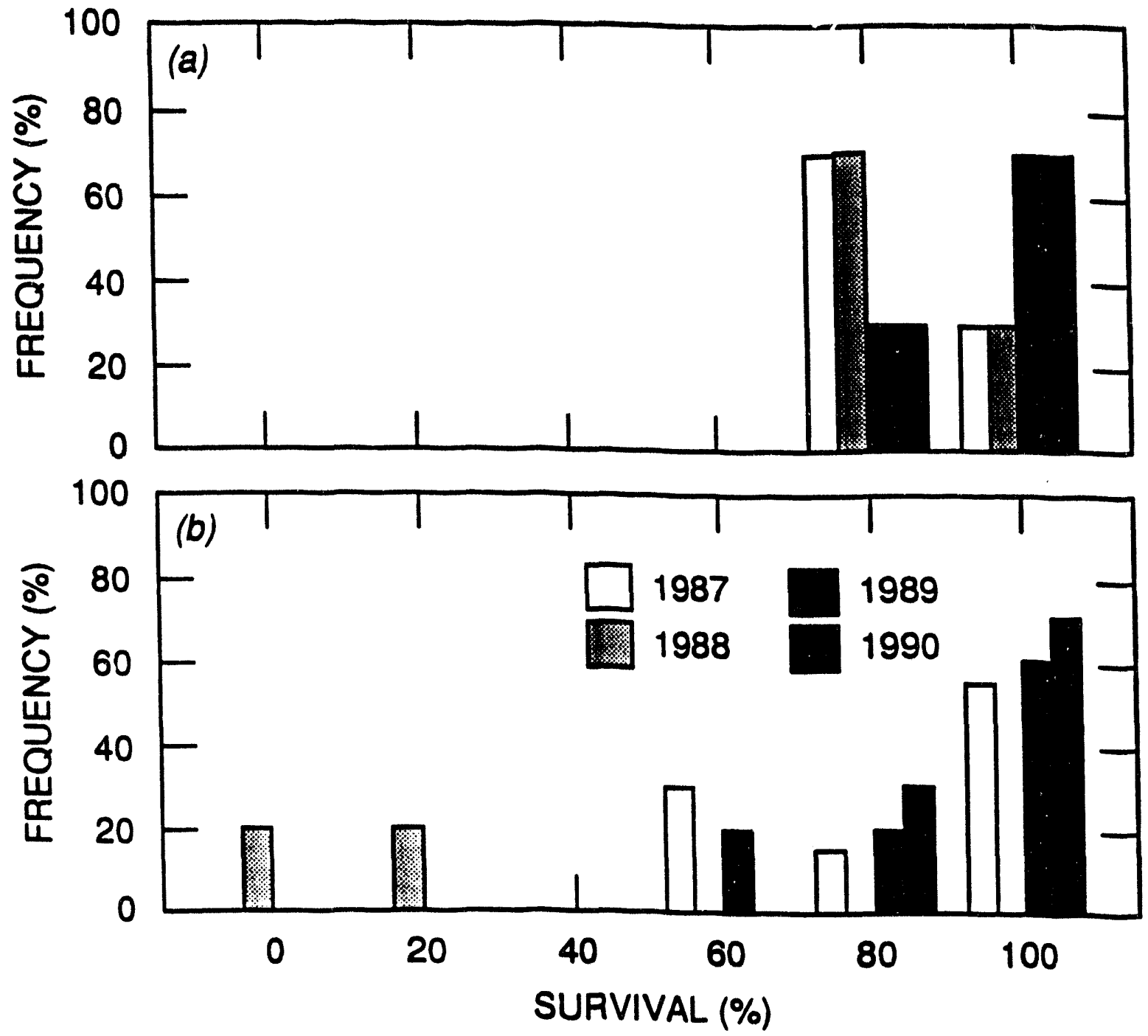

Fig. 3.6. Frequency distribution of fathead minnow survival at Mitchell Branch kilomater (MIK) 0.12 (a) and MIK 0.45 (b), 1987-90. 
through 3.8) show that acute toxicity (survival 60\%) was detectable at MIKs 0.45, 0.54, and 0.71 but declined after 1988. Mean survival of minnows in tests of water from MIK 0.12 was never $<80 \%$. At MIK 0.45 , acute toxicity was demonstrated in $40 \%$ of the tests in 1988; in 1989 and 1990 , no acute toxicity was found. At MIK 0.54, acute toxicity was found in $60 \%$ of the tests in 1988 and $17 \%$ in 1989; in 1987 and 1990 , no acute toxicity was found. Water from MIK 0.71 was typically very toxic to fathead minnows. In 1987, 1988, and 1989, acute toxicity was found in 70,80 , and $30 \%$ of the tests, respectively, but in 1990 , water from MIK 0.71 was not acutely toxic to fish in any test. Low survival was occasionally seen at MIK 1.0 and was frequently observed at MIK 1.43 . However, this was probably not caused by a toxicant per se.

ORNL-DWG 90M-16805

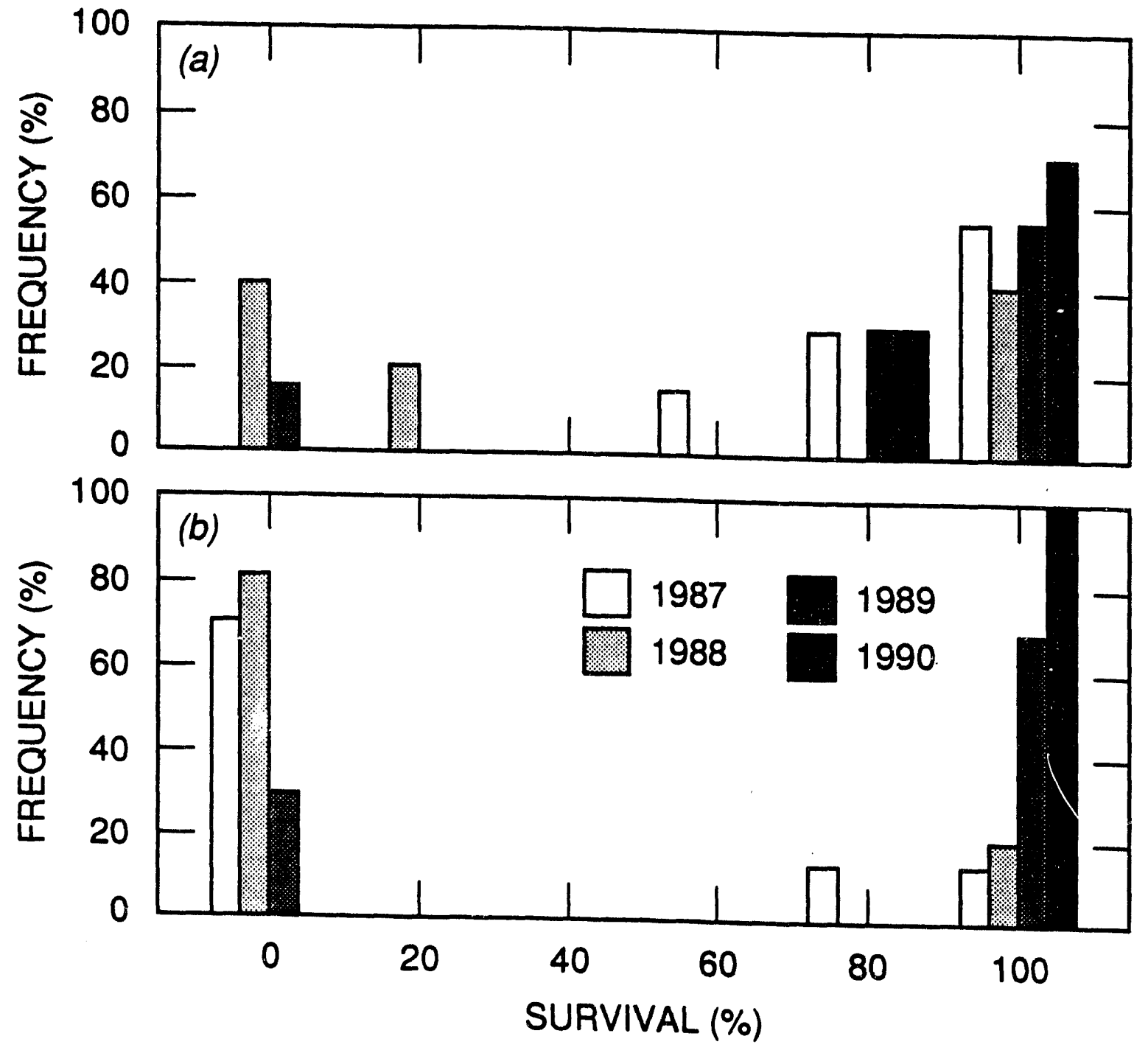

Fig. 3.7. Frequency distribution of fathead survival at Mitchell Branch kilometer (MIK) 0.54 (a) and MIK 0.71 (b), 1987-90. 


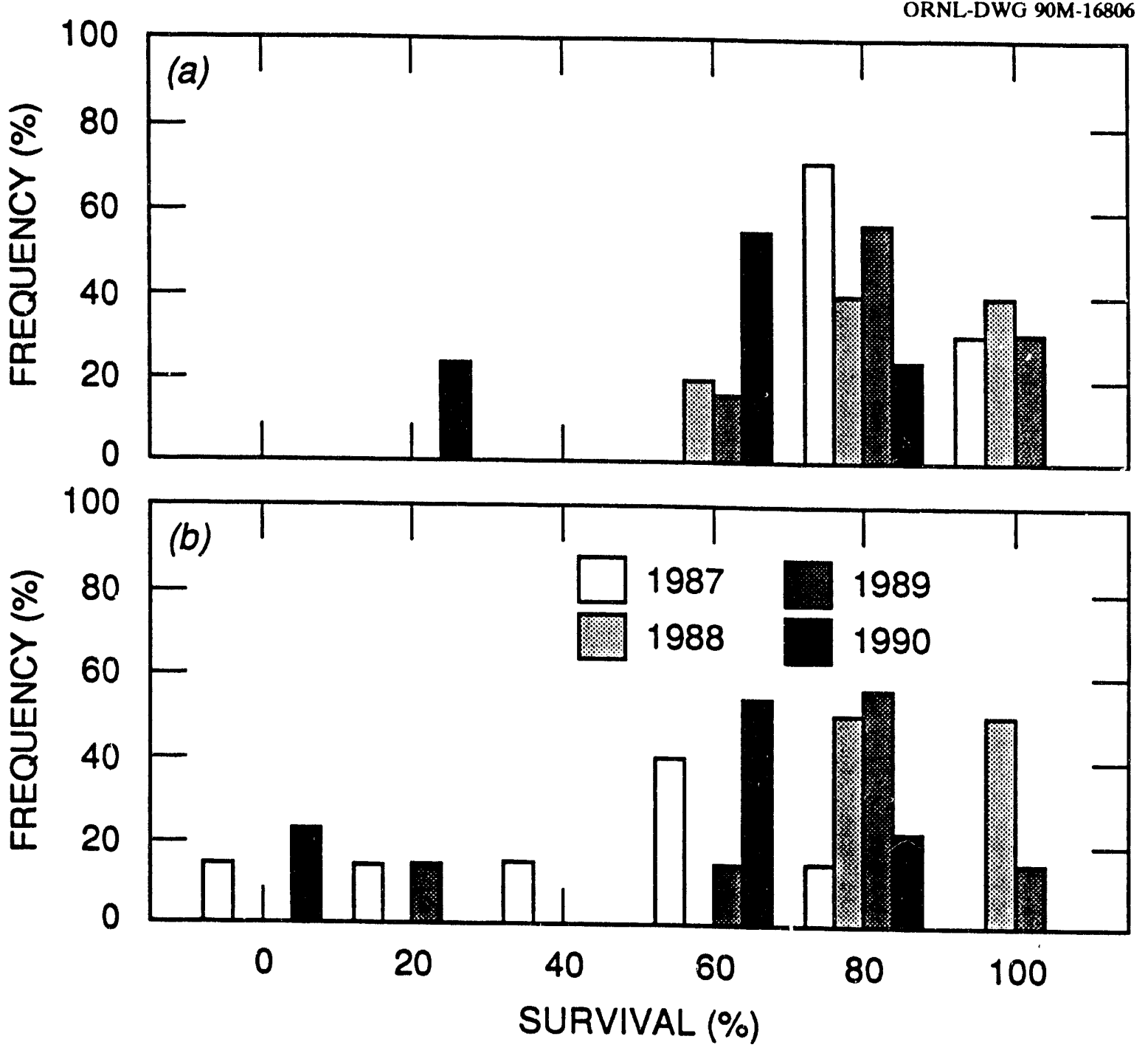

Fig. 3.8. Frequency distribution of fathead survival at Mitchell Branch kilometer (MIK) 1.0 (a) and MIK 1.43 (b). 1987-90.

To evaluate the presence of chronic toxicity (significant reduction in growth compared with the control) at each site, separate ANOVAs were conducted for each test. Only sites where mean survival was $>60 \%$ were included. Results of this analysis (Table 3.11) showed that chronic toxicity was evident only at MIK 0.54 but at a very low frequency. In contrast, water from MIKs $0.71,0.54,0.45$, and 0.12 was more often acutely toxic (survival $<60 \%$ ) or not toxic at all.

\subsubsection{Ceriodaphnia tests}

Mean survival and reproduction of Ceriodaphnia for each site in each year are plotted in Fig. 3.9. Differences in survival among years and sites could not be effectively determined by means of an ANOVA because most values had a bimodal distribution, indicating an all-or-none type of response. However, survival was reduced every year at MIKs $0.71,0.54$, and 0.45 compared with 1.43 . In general, mean 
Table 3.11. Percentage of fathead minnow tests at each site where no toxicity, acute toxicity, or chronic toxicity was observed

\begin{tabular}{cccc}
\hline Site & $\begin{array}{c}\text { No toxicity } \\
(\%)\end{array}$ & $\begin{array}{c}\text { Acute toxicity } \\
(\%)\end{array}$ & $\begin{array}{c}\text { Chronic toxicity } \\
(\%)\end{array}$ \\
\hline MIK 0.71 & 50 & 50 & 0 \\
MIK 0.54 & 68 & 27 & 5 \\
MIK 0.45 & 82 & 8 & 0 \\
MIK 0.12 & 100 & 0 & 0 \\
\hline
\end{tabular}

aSurvival was $\geq 60 \%$ and no reduction in growth.

'Survival was $<60 \%$.

'Survival was $\geq 60 \%$ and growth was significantly reduced compared with the control.

Note: MIK $=$ Mitchell Branch kilometer.
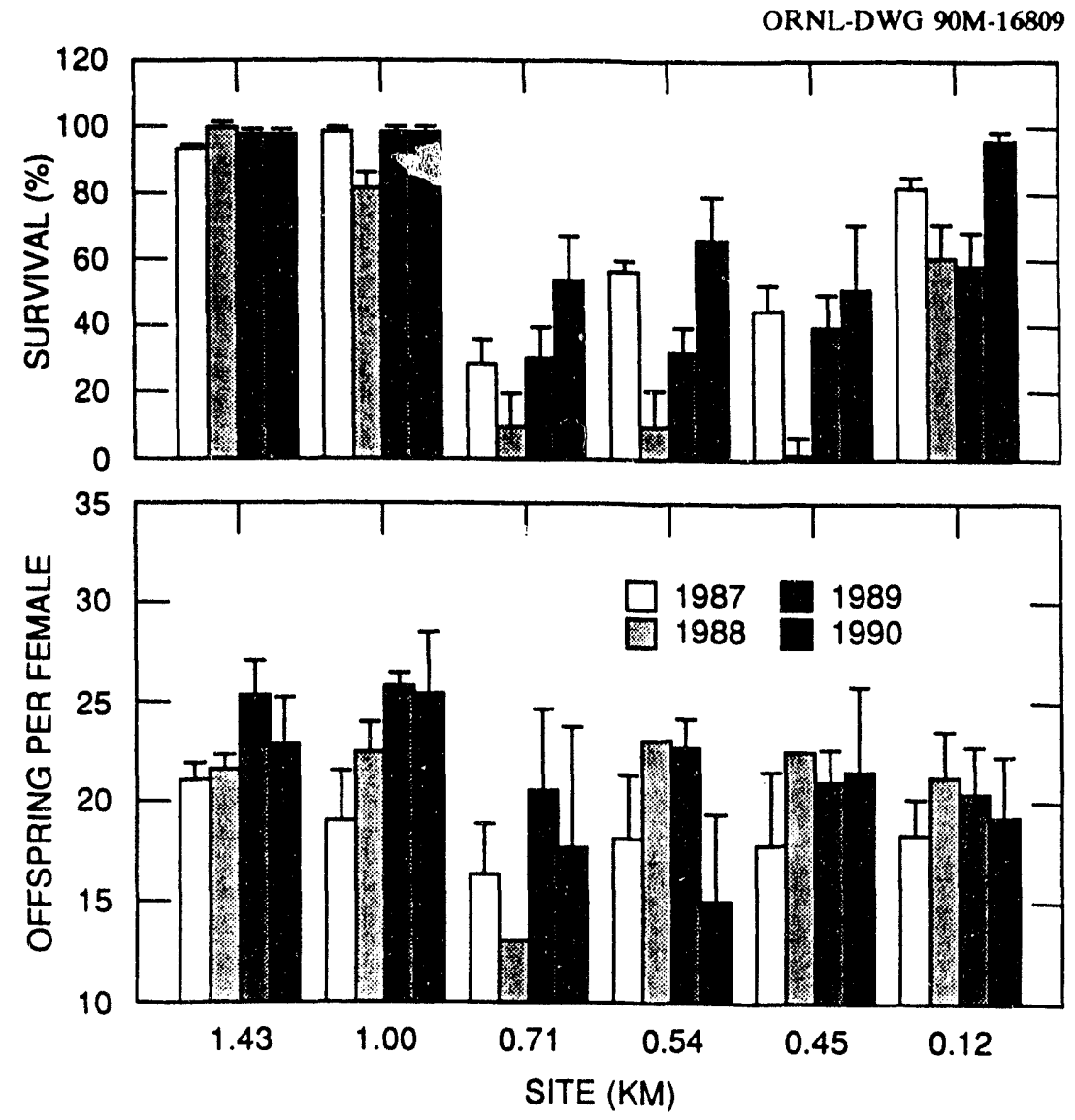

Fig. 3-9. Mean survival and fecundity of Ceriodaphnia for each site in Mitchell Branch, 1987-90. Vertical bars represent \pm 1 SE. 
survival increased with distance downstream from MIK 0.71 , reaching a peak at MIK 0.12. In 1988 and 1989, mean fecundity at MIKs $0.71,0.54,0.45$, and 0.12 was also reduced compared with that at MIK 1.43. In 1990, fecundity at MIKs 0.71 and 0.54 were reduced compared with that at MIK 1.43.

Frequency distributions of survival of Ceriodaphnia at each site for each year show that acute toxicity was present at MIKs $0.71,0.54,0.45$, and 0.12

(Figs. 3.10 through 3.12). As for fathead minnows, the incidence of acute toxicity (survival $<60 \%$ ) declined after 1988. Water from MIK 0.12 was acutely toxic to Ceriodaphnia during 1988 and 1989 but not in 1990. At MIKs 0.45 and 0.54 , acute toxicity was found in 22 to $80 \%$ of the tests conducted each year. At MIK 0.71, the incidence of acute toxicity was higher, occurring in 40 to $80 \%$ of the tests each year.

Except for one test in 1988, survival was always been $\geq 80 \%$ at MIKs 1.0 and 1.43 .

To evaluate the presence of chronic toxicity (i.e., a significant reduction in reproduction compared with that at MIK 1.43) at each site, separate ANOVAs were conducted for each test. Only those sites where mean survival was $>60 \%$

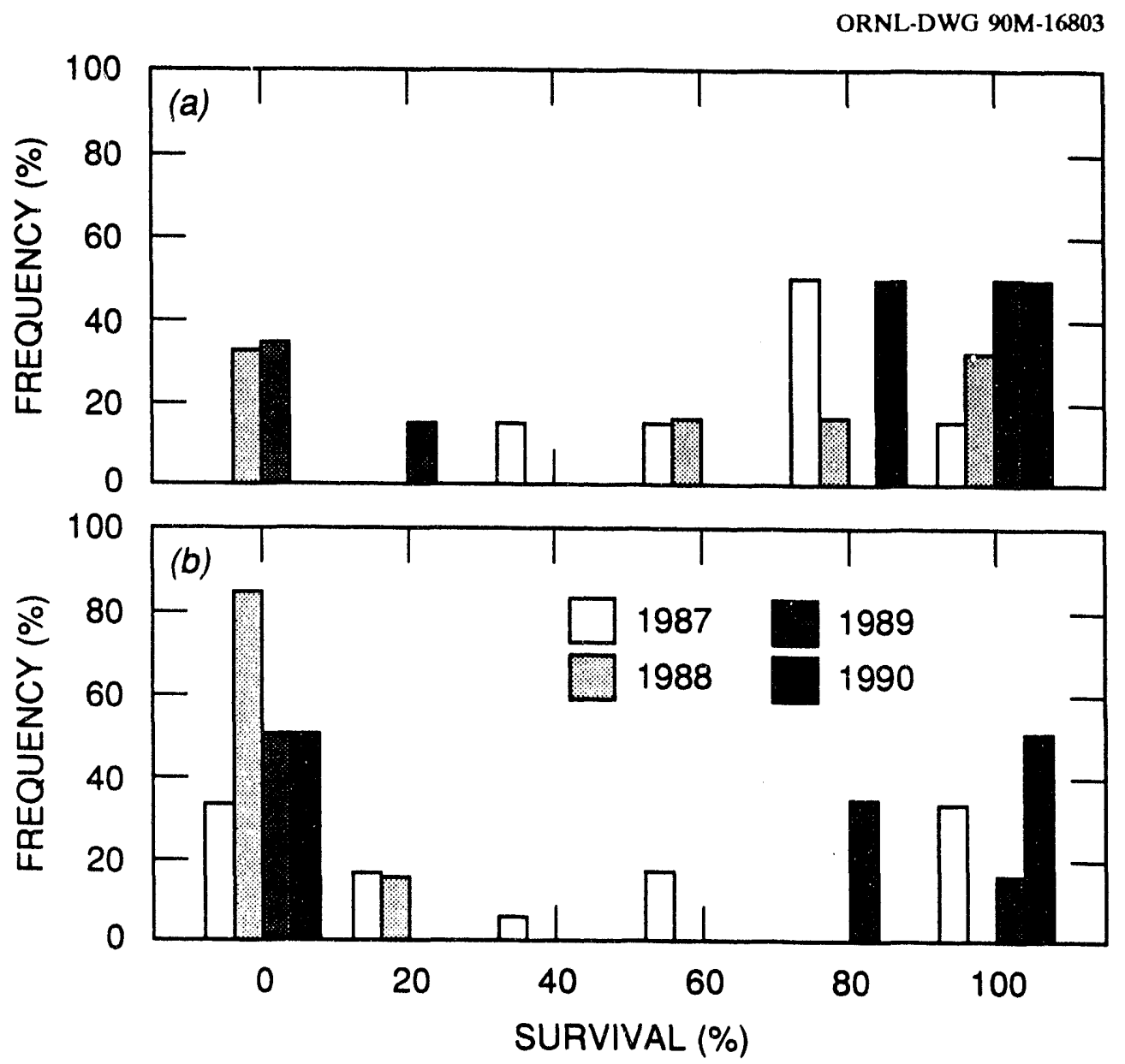

Fig. 3.10. Frequency distribution of Ceriodaphnia survival at Mitchell Branch kilometer (MIK) 0.12 (a) and MIK 0.45 (b), 1987-90. 


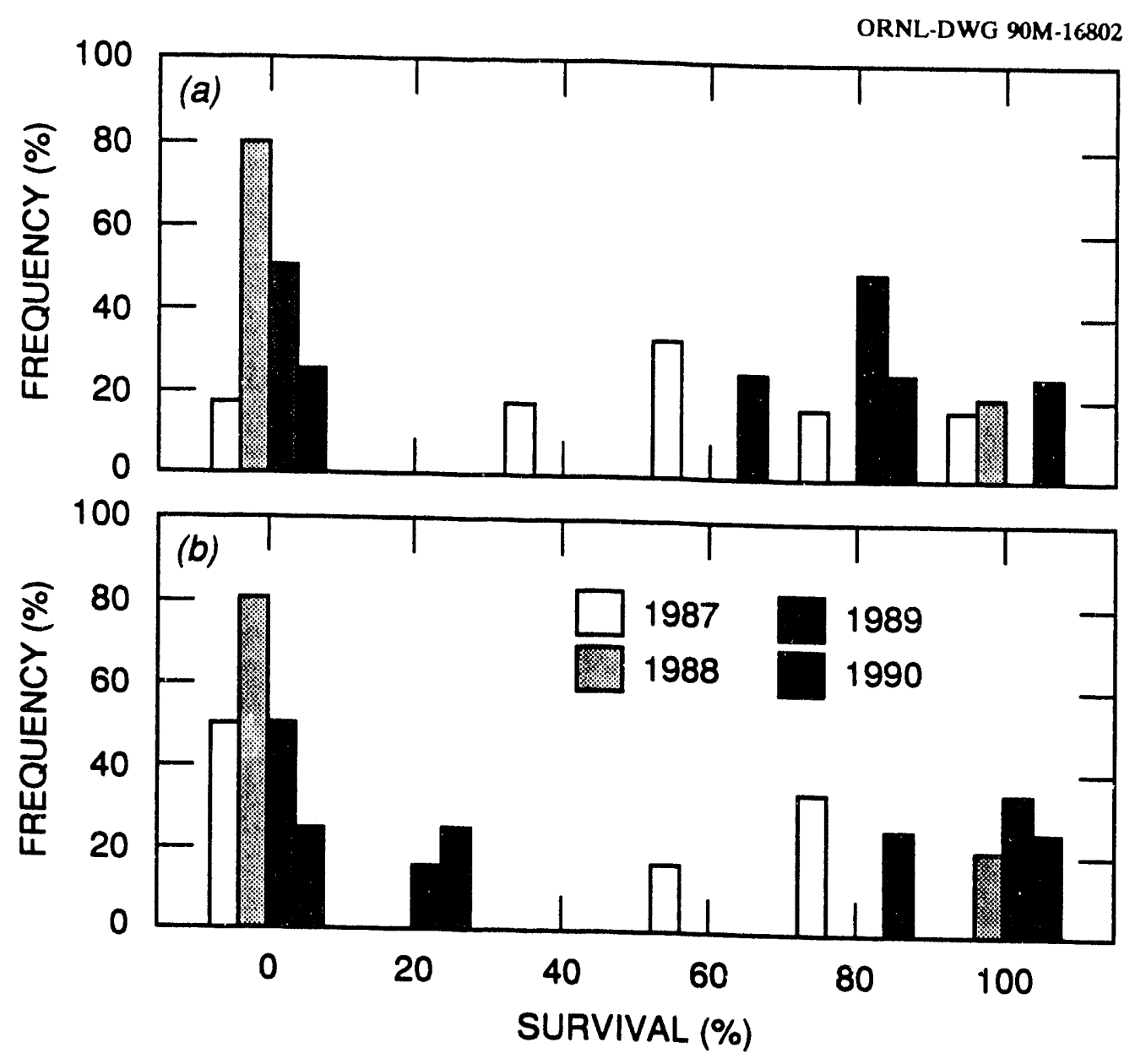

Fig. 3.11. Frequency distribution of Ceriodaphnia survival at Mitchell Branch kilometer (MIK) 0.54 (a) and MIK 0.71 (b), 1987-90.

were included. Results of this analysis (Table 3.12) show that chronic toxicity was evident at MIKs $0.71,0.54$, and 0.12 . For example, in $23 \%$ of the tests conducted with water from MIK 0.12, Ceriodaphnia survival was $\geq 60 \%$ but fecundity was significantly reduced compared with that at MIK 1.43.

\subsubsection{Discussion}

A noticeable improvement in the water quality of Mitchell Branch has occurred since the end of 1988 . Conductivity and hardness of the water at and below MIK 0.71 remained elevated relative to the two sites furthest upstream, indicating impact from the K-1407-E/F ponds. However, the magnitude of increases in these parameters with distance downstream has declined since 1988. The toxicity tests also documented that water quality improved during 1989 and 1990. After 1988, fathead minnow survival was no longer adversely affected by water from the midreach section of Mitchell Branch. Survival of Ceriodaphnia continued to be low in this section of the stream in 1989 and 1990 because of high concentrations of TRC, but the frequency of acute toxicity was not as high as in 1987 and 1988. This 


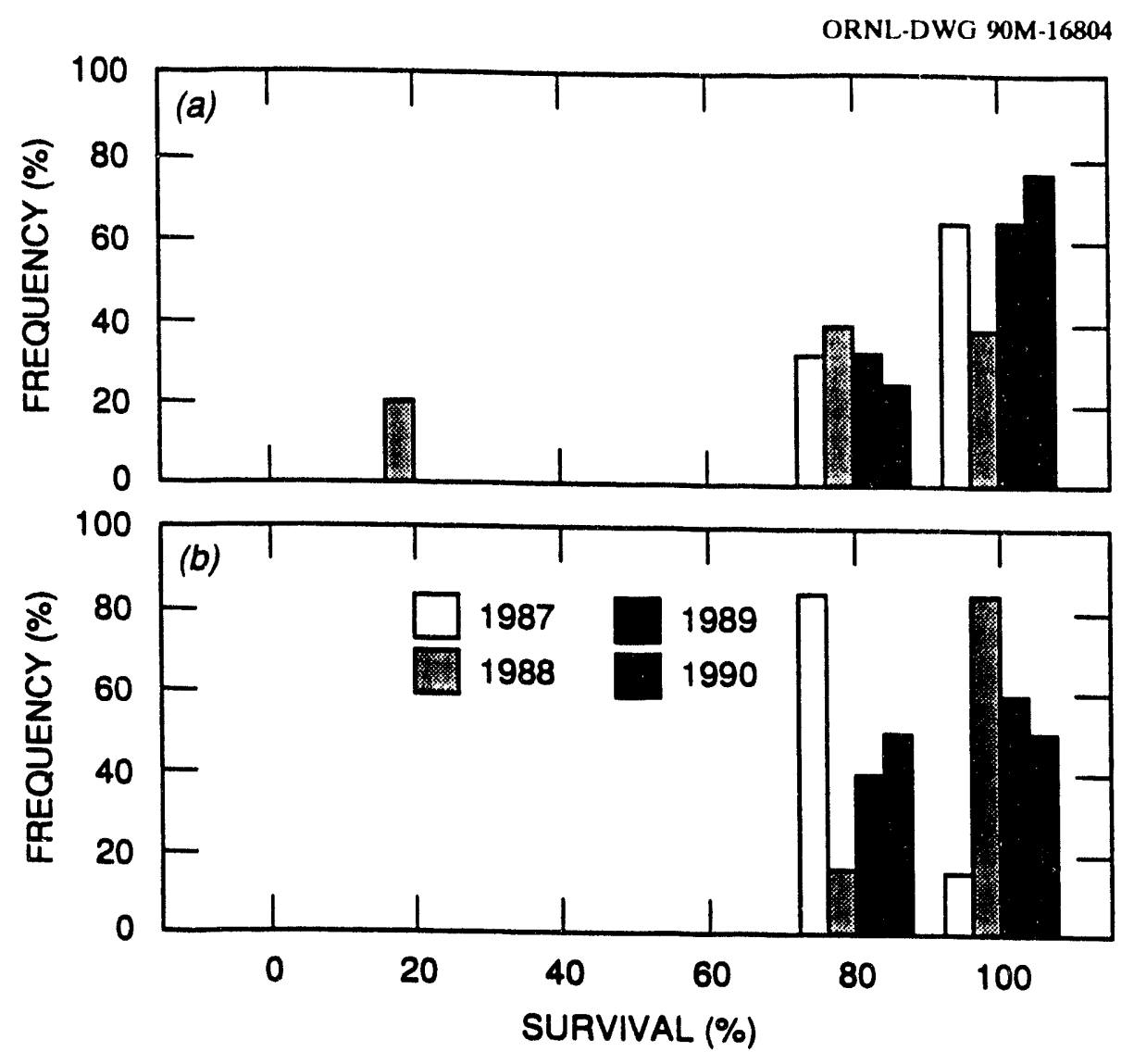

Fig. 3.12. Frequency distribution of Ceriodaphnia survival at Mitchell Branch kilometer (MIK) 1.0 (a) and MIK 1.43 (b), 1987-90.

Table 3.12. Percentage of Ceriodaphnia tests at each site where no toxicity, acute toxicity, or chronic toxicity was observed

\begin{tabular}{lccc}
\hline Site & $\begin{array}{c}\text { No toxicity } \\
(\%)\end{array}$ & $\begin{array}{c}\text { Acute toxicity } \\
(\%)\end{array}$ & $\begin{array}{c}\text { Chronic toxicity } \\
(\%)\end{array}$ \\
\hline MIK 0.71 & 23 & 64 & 13 \\
MIK 0.54 & 36 & 55 & 9 \\
MIK 0.45 & 41 & 59 & 0 \\
MIK 0.12 & 59 & 18 & 23 \\
\hline
\end{tabular}

${ }^{a}$ Ceriodaphnia survival was $\geq 60 \%$ and no reduction in fecundity.

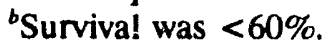

${ }^{c}$ Survival was $\geq 60 \%$ and reproduction was significantly reduced compared with MIK 1.43 .

Note: MIK = Mitchell Branch kilometer. 
was probably because of fewer excursions in TRC concentrations above $0.20 \mathrm{mg} / \mathrm{L}$ (Figs. 3.3 and 3.4). It was difficult to determine if the improvements in water quality were the result of the closure of K-1407-B pond (October 1988) or an increase in streamflow (thus, greater dilution) during 1989 and 1990 . (See Sect. 2.1.1 for flow data.) Changes in water quality caused by the closure of $\mathrm{K}-1407-\mathrm{B}$ pond were evident in terms of lower concentrations of chloride, sulfate, and dissolved solids at MIK 0.54 (Tables B.2 and B.3). Fewer excursions of high TRC at all sites were probably the result of higher streamflows during 1989 and 1990 because no known process changes occurred during this period. Thus, the closure of the K-1407-B pond in 1988 and the end of the drought in late 1988 both contributed to the improved water quality of Mitchell Branch. There continues to be evidence of chronic toxicity in

Mitchell Branch: Ceriodaphnia fecundity was reduced (compared with that at MIK 1.43) in some tests at MIKs 0.71 , 0.54 , and 0.12 when survival was $\geq 60 \%$. This toxicity may be the result of low levels of TRC from the storm drains, constituents released from the $\mathrm{K}-1407-\mathrm{E} / \mathrm{F}$ ponds, and/or area-source contamination.

However, measurements of the major metals, anions, and cations in the stream water have not yet revealed a probable toxicant.

The cumulative frequency distribution of TRC in Mitchell Branch and the occurrence of acute toxicity ( $0 \%$ survival) at each site provide a means to estimate the concentration of TRC that can cause acute toxicity in Mitchell Branch. The frequency of acute toxicity at a particular site should coincide with the concentration of TRC that is acutely toxic. For example, survival of Ceriodaphnia was $0 \%$ in $48 \%$ of the toxicity tests at MIK 0.71 (Fig. 3.11). By using the cumulative frequency of TRC at MIK 0.71 (Fig. 3.2), the TRC concentration was calculated to be greater than $0.18 \mathrm{mg} / \mathrm{L}$ about $48 \%$ of the time. Thus, when the TRC at MIK 0.71 is $\geq 0.18 \mathrm{mg} / \mathrm{L}$, the water should be acutely toxic to Ceriodaphnia. If the same rationale is used for each site, the concentration of TRC predicted to be acutely toxic to Ceriodaphnia ranged from about 0.08 to $0.15 \mathrm{mg} / \mathrm{L}$ at MIKs 0.12 , $0.45,0.54$, and 0.71 . The concentration of TRC predicted to cause acute toxicity to fathead minnows ranged from about 0.2 to $0.5 \mathrm{mg} / \mathrm{L}$ at the same sites. It is not possible to predict the chronic toxicity of TRC because of the static-renewal nature of the toxicity test.

Mean survival of fathead minnow larvae in water from the uncontaminated reference site (MIK 1.43) continued to be low. As stated in the First Annual BMAP report (Smith et al. 1988), this is probably caused by a pathogen in the water.

\subsection{FUTURE STUDIES}

The results of effluent and ambient toxicity tests show the need for continued monitoring of effluents and Mitchell Branch. Monitoring of the storm drains should be continued because they are a major source of TRC to the stream and they may contain other toxicants as well. Discharge from the $\mathrm{K}-1407-\mathrm{E} / \mathrm{F}$ ponds is expected to be discontinued in 1993 . To continue to document improvements in water quality as the ponds are closed and as reductions are made in the release of chlorine, monitoring will continue on a bimonthly basis. Because fathead minnows are typically insensitive to effluent from the $\mathrm{K}-1407-\mathrm{E} / \mathrm{F}$ ponds and the K-1407-J basin and to Mitchell Branch water, depending on the approval of the Oak Ridge K-25 Site and the Tennessee Department of Environment and Conservation, these effluents and ambient sites will be evaluated with Ceriodaphnia only. 


\title{
4. BIOACCUMULATION STUDIES
}

\author{
M. J. Peterson and G. R. Southworth
}

The primary objectives of contaminant monitoring in Mitchell Branch biota are to (1) identify substances that accumulate to levels exceeding those observed in biota from nearby uncontaminated reference streams and (2) evaluate the extent and significance of contamination by those substances in Mitchell Branch and downstream aquatic systems. Secondary objectives are to assist in locating sources of contaminants that accumulate to unacceptable levels and to evaluate the relative importance of current vs past discharges in determining contaminant levels in biota.

\subsection{INTRODUCTION}

Results presented in the first report for Mitchell Branch (Smith et al. 1993) demonstrated that $\mathrm{Hg}$ and PCBs accumulate to levels significantly above background in the biota. Whether or not Mitchell Branch was a significant source of the elevated mercury levels in its fish or in fish from lower Poplar Creek was unclear. PCB monitorin! data from clams were more conclusive and clearly documented that Mitchell Branch is a source of PCBs. The data also suggested that the stream may be a significant source of PCB contamination to biota in lower Poplar Creek.

Monitoring of metals and organics in Mitchell Branch biota continued on about a yearly basis between May 1987 and March 1990. To better assess possible contributions of mercury and PCBs from Mitchell Branch to downstream waters, sunfish sampling was expanded to include a number of sites in Poplar Creek and the
Clinch River in 1987 and 1988 as part of a coordinated effort between ORNL, the Oak Ridge Y-12 Plant, and the Oak Ridge K-25 Site biomonitoring programs. Caged clams (Corbicula fluminea) were used in Poplar Creek in 1988 to gain additional information on the importance of Mitchell Branch as a source of PCBs to Poplar Creek. In coordination with the ORNL BMAP, channel catfish (Ictalurus punctatus) were collected from Poplar Creek and the Clinch River and analyzed for PCBs in 1988 and 1989. Channel catfish probably accumulate the highest concentrations of PCBs attainable in a sport species in Poplar Creek that could be influenced by PCBs from Mitchell Branch. In 1988, gizzard shad (Dorosoma cepedianum) were also collected and analyzed for PCBs from Poplar Creek in an effort to evaluate the role of forage fish in PCB dispersal.

\subsection{METHODS}

Fish were collected by electrofishing from the lower reaches of Mitchell Branch (MIK 0.2) beginning in May 1987 and continuing approximately yearly through 1990 (Fig. 2.1). Approximately 12 redbreast sunfish (Lepomis auritus) were collected from Mitchell Branch in each sampling period. Attempts were made to obtain eight fish samples for mercury analysis, eight for PCB analysis, and four for metals other than mercury. Early attempts to collect fish from Mitchell Branch, however, revealed that the stream did not support an adequate population of adult fish to meet the full sampling 
requirements of the contaminant monitoring program. Thus, because of the small size of the few redbreast sunfish present in the stream in 1987 and 1988, only analyses of mercury and other metals were conducted in those years. In 1989 and 1990 , however, enough adult redbreast sunfish were collected to include an analysis for PCBs. Analysis of metals (other than mercury) was not performed on Mitchell Branch fish in 1989.

Bluegill (Lepomis macrochinus) were routinely collected for mercury and PCB analysis from various sites on Poplar Creek and the Clinch River from 1987 to 1990 (Figs. 2.1 and 2.2). Fish from Poplar Creek and the Clinch River were sampled to evaluate the relative importance of mercury and $\mathrm{PCB}$ inputs from multiple sources (Bear Creek, East Fork Poplar Creek, Mitchell Branch, and White Oak Creek Watershed) that enter this system. Eight bluegill sunfish were collected by electrofishing from each of four sites on Poplar Creek (PCKs 10.4, 8.2, 6.9, and 2.1, Figs. 2.1 and 2.2) and the Clinch River (CRK 15.0) in November/December of 1987 and 1988. When possible, collections were restricted to individuals of a size likely to be taken by sport fishermen $(>50 \mathrm{~g})$. Sites on Poplar Creek immediately above (PCK 8.2) and below (PCK 6.9) Mitchell Branch were resampled for bluegill in March 1990. Reference fish for the Poplar Creek and Mitchell Branch collections were obtained from Hinds Creek (1985-89) and Melton Hill Reservoir (MHR; 1987-88) in Anderson County, Tennessee (Fig. 2.1).

Upon collection, sunfish from all sites were tagged with a unique four-digit tag wired to the lower jaw and placed on ice in a labelled ice chest; the chest was locked for transport to the laboratory. Upon return to the laboratory, each fish was weighed and measured (total length), and scale samples were taken for age determination. The fish were then fileted and skinned, and a 1- to 2-g portion of the anterior dorsal axial muscle filet was excised for mercury determination, if a 5-g portion of tissue was also available, it was used for analysis of PCBs and/or other metals. Samples were wrapped in heavy-duty aluminum foil, labelled, and stored at $-20^{\circ} \mathrm{C}$ in a locked freezer in Building 1505 at ORNL until delivered to the Analytical Chemistry Division (ACD) laboratory at ORNL. Channel catfish (Ictalurus punctatus) were collected for PCB analysis from PCK 6.9 and CRK 15.0 by means of trotlines in the summers of 1988 and 1989. Upon collection, the channel catfish were handled and processed as sunfish were, except that: (1) the dorsal spine was taken for age determination and (2) fish filets were frozen and ground three times in a hand-powered meat grinder before a 10to 20-g sample was removed for analysis.

In the summer of 1988, gizzard shad (Dorosoma cepedianum) were collected for PCB analysis by electrofishing at the same sites as catfish. Three composite samples of five shad each (22-29 cm total length) were obtained at each site. Each shad composite was wrapped in aluminum foil and placed on ice in the field. Upon return to the laboratory, the digestive tract was removed from each fish and the composite frozen. Later, each frozen composite was ground three times in the meat grinder before a 10 - to $20-\mathrm{g}$ sample was removed for analysis.

Caged clams (Corbicula fluminea) were placed in Mitchell Branch (MIK 0.2) to monitor for organic contaminants on an approximately annual basis from 1987 to 1990. In 1988, clams were placed at PCKs 8.2 and 6.9 (upstream and downstream of Mitchell Branch, respectively) to monitor for PCBs in Poplar Creek. Clams were obtained in March 1987 from Beaver Creek near Karns in Knox County, Tennessee; in May 1988 from Bull Run Creek in Union County, Tennessee; and 
in July 1989 from Paint Rock Creek in Meigs County, Tennessee. After the clams were held for $24 \mathrm{~h}$ in the laboratory in dechlorinated process water, they were put in polypropylene cages that were held securely at each site. One set of clams from the reference stream was frozen immediately for analysis as a control. Each cage placed in a stream held $\sim 50$ clams; each clam contained 0.5 to $2 \mathrm{~g}$ (wet weight) of soft tissue. The cages remained at the sites for 4 weeks, after which the clams were removed, placed on ice in a locked cooler, transported to the laboratory, and deposited in a locked freezer in Building 1505 at ORNL. After the clams were frozen, the shells were removed and the frozen soft tissue was placed in a $20-\mathrm{ml}$ glass vial. Composite samples weighing $\sim 5$ and $10 \mathrm{~g}$ each were obtained for PCB analysis and gas chromatographic/mass spectrometric analysis respectively. Samples were refrozen prior to delivery to the ORNL ACD laboratory for chemical analysis.

Mercury determinations were carried out by the $A C D$ recording to procedure EC 420 (Martin Marietta Energy Systems 1983). Samples were digested in a mixture of nitric acid, perchloric acid, and potassium dichromate, after which the mercury was reduced with stannous chloride and determined by cold vapor atomic adsorption spectrophotometry. Samples collected in 1987 and 1988 were analyzed for metals by graphite furnace atomic adsorption spectrophotometry following digestion with concentrated nitric acid (EPA 1980). Samples obtained in 1990 were analyzed for metals by inductively coupled argon plasma emission spectroscopy or by inductively coupled plasma mass spectrometry following digestion with concentrated nitric acid (EPA 1980). PCBs were determined by packed-column gas chromatography following methylene chloride extraction and adsorption column cleanup (EPA 1980). Organic priority pollutants were analyzed by procedure PPB 12/83 (EPA 1983 ), in which the homogenized sample is extracted in methylene chloride, cleaned up by using column chromatography, and analyzed by capillary column gas chromatography with mass spectrometric or electron capture detectors.

Statistical evaluations of the data were made by using SAS procedures and software (SAS 1985a, 1985b) for the ANOVA, Duncan's multiple range test, t-test, linear regression analysis, and the calculation of means, standard deviations, standard errors, and coefficients of variation. Quality assurance was maintained by using a combination of (1) blind duplicate analyses; (2) split-sample analyses between the EPA Environmental Services Laboratory, Athens, Georgia, and the ACD Laboratory at ORNL; and (3) the analyses of biological reference standards and uncontaminated fish. Recoveries of PCBs were verified by analysis of uncontaminated fish or clam samples spiked with known amounts of PCBs. Details and results of $\mathrm{QA}$ analyses are summarized in Appendix $\mathrm{C}$ and results of tissue analyses are tabulated in Appendix D.

\subsection{RESULTS AND DISCUSSION}

\subsubsection{Mercury in Mitchell Branch Fish}

Mercury was clearly elevated in fish from Mitchell Branch relative to fish from the reference stream (Table 4.1). However, the difference was not large, and levels were well below the Food and Drug Administration (FDA) action level of $1.0 \mu \mathrm{g} / \mathrm{g}$ (FDA 1984a). No fish from Mitchell Branch exceeded the more conservative Preliminary Guidance Value (PGV) for $\mathrm{Hg}$ of $0.42 \mu \mathrm{g} / \mathrm{g}$ (Travis et al. 1986).

The mean mercury levels in Mitchell Branch redbreast varied among years, with the highest mean $\mathrm{Hg}$ concentration in fish 
Table 4.1. Mercury concentrations (in micrograms per gram, wet weight) in blucgill (Lepomis macrochinus) and rodbreast sunfish ( $L$ auritus) collectod at sitcs in the Poplar Creck drainage area in the vicinity of the Oak Ridge K-25 Site

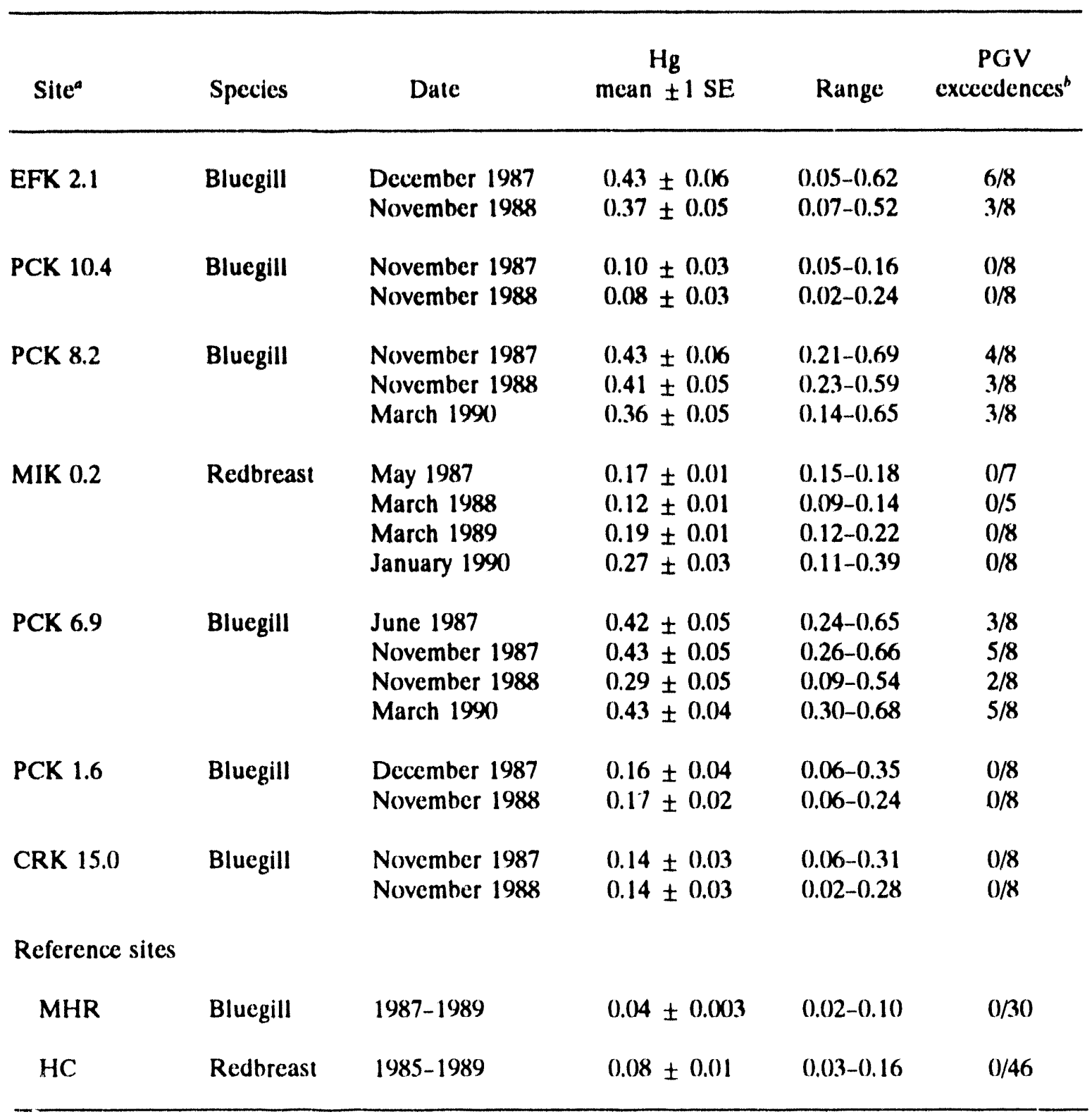

${ }^{\circ}$ MHR = Melton Hill Reservoir; HC = Hinds Creek; EFK = East Fork Poplar Creck kilometer; PCK = Poplar Creek kilometer; MIK = Mitchell Branch kilometer; CRK $=$ Clinch River kilometer.

${ }^{b}$ Preliminary Guidance Value. Number of samples exceeding the PGV value for $\mathrm{Hg}$ of $0.42 \mu \mathrm{g} / \mathrm{g}$ (C. C. Travis et al. 1986, Preliminary reviews of TVA fish sampling and analysis report, Report of Task Group Five 10 Oak Ridge Task Force, Oak Ridge National Laboratory, Oak Ridge, Tenn., Mimeo, Jan. 1986) divided by the total number of samples. No fish exceeded the FDA action limit of $1.0 \mu \mathrm{g} / \mathrm{g}$ (U.S. Department of Agriculture Food and Drug Administration (FDA), 1984 Action level for methylmercury in fish, Fed. Regst. 49(224):45663. 
occurring in the most recent (1990) sampling period. However, accumulation of $\mathrm{Hg}$ in fish tends to increase with body size. A significant positive relationship was observed between $\mathrm{Hg}$ concentration in fish tissue and fish weight (linear regression analysis, $p<0.05$ ) in Mitchell Branch. Thus, the larger fish collected in Mitchell Branch in 1989 and 1990 (mean weight of 26 and $41 \mathrm{~g}$, respectively) would be expected to contain higher concentrations of mercury than the extremely small fish collected in 1987 and 1988 (mean weight of $5 \mathrm{~g}$ in both years).

Results previously reported for Mitchell Branch showed that sunfish collected from this stream had accumulated elevated levels of $\mathrm{Hg}$ (Smith et al. 1993). However, it was not clear from the data whether the source of mercury was Mitchell Branch or whether these fish had accumulated it while in another stream (i.e., Poplar Creek or EFPC) before immigrating into Mitchell Branch. By comparing mercury concentrations of fish from Mitchell Branch with those of fish from Poplar Creek, it may be possible to determine the extent to which Mitchell Branch serves as a source of mercury in fish. Although these comparisons are for two different species of sunfish (redbreast sunfish and bluegill), past studies (Loar 1992b; Loar 1993a) have shown no significant differences in mercury concentrations between the two species where the concentrations in fish are $\sim 0.5 \mu \mathrm{g} / \mathrm{g}$ or less. The mean mercury concentration in Mitchell Branch fish $(0.20 \mu \mathrm{g} / \mathrm{g})$ was approximately half that in fish $(0.39 \mu \mathrm{g} / \mathrm{g})$ at sites on Poplar Creek nearest Mitchell Branch (PCKs 8.2 and 6.9) in all years. However, because there is a positive relationship between mercury concentrations in Mitchell Branch fish and fish weight, adult fish, equal in weight to Poplar Creek fish (approximate mean weight of $70 \mathrm{~g}$ ), would be expected to contain much higher levels of mercury, if they were present.

Linear regression of mercury vs weight for MIK 0.2 sunfish yielded the expression $\mathrm{Hg}=0.13+0.00285$ (wt), $\mathbf{R}^{2}=0.69$. When this expression was used to estimate the concentration of mercury in a hypothetical $70-\mathrm{g}$ fish from Mitchell Branch, a value of $0.33 \mu \mathrm{g} / \mathrm{g}$ was obtained. This value is very similar to the mean concentration in Poplar Creek fish of $0.39 \mu \mathrm{g} / \mathrm{g}$. These results suggest that fish collected from Mitchell Branch may have accumulated their mercury burden from Poplar Creek before immigrating into Mitchell Branch. Conversely, a single creek chub (Semotilus atromaculatus) collected from Mitchell Branch, which was unlikely to have come from Poplar Creek, was analyzed for mercury in 1987 (Smith et al. 1993) and found to contain a substantial mercury concentration $(0.29 \mu \mathrm{g} / \mathrm{g})$. Elevated mercury levels in Mitchell Branch sediments (Ashwood et al. 1986) and water (Smith et al. 1993) also provide strong evidence that Mitchell Branch is a source of mercury to its biota. Because of this conflicting evidence, the significance of Mitchell Branch as a source of the elevated mercury levels in its fish remains unresolved.

\subsubsection{Mercury in Poplar Creck Fish}

Mercury concentrations were measured in bluegill from several sites in Poplar Creek and the Clinch River to assess the relative importance of various streams (Bear Creek, EFPC, Mitchell Branch, and White Oak Creek) to the system. Results for the Poplar Creek area are shown in Table 4.1; results for the entire reservation are shown in Figs. 4.1 and 4.2.

The most upstream site on Poplar Creek (PCK 10.4) was upstream of an, 
RNL.DWG $88 M-15410 \mathrm{R}$

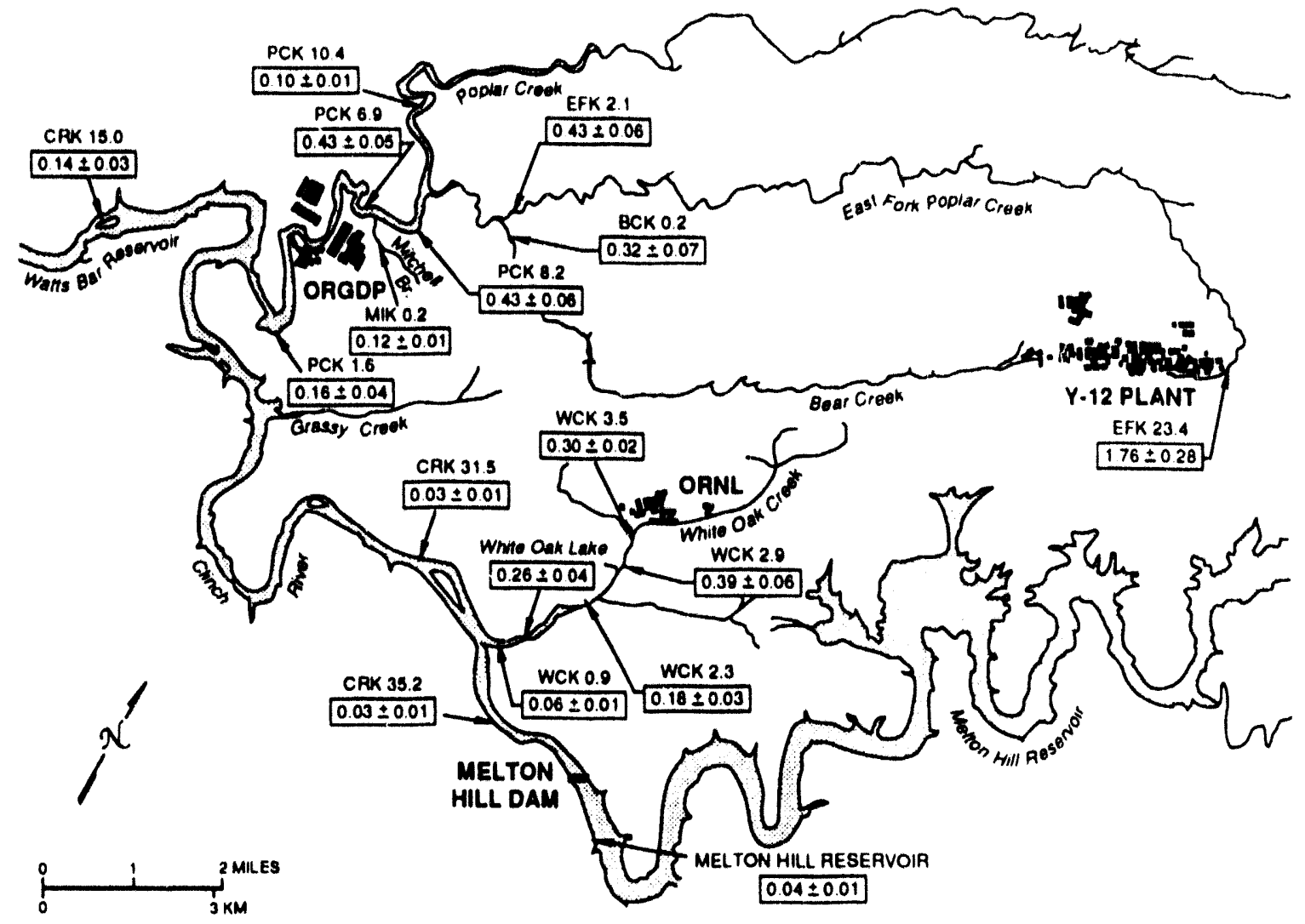

Fig. 4.1. Mean concentrations ( \pm 1 SE) of mercury (in micrograms per gram, wet weight) in bluegill $(n=8)$ collected in fall/winter 1987 at sites on the Oak Ridge Reservation and nearby reaches of the Clinch River. Rockbass (Ambloplites rupestris) and redbreast sunfish (Lepomis auritus) were substituted for bluegill at Bear Creek kilometer 0.2 and Mitchell Branch kilometer 0.2 respectively.

discharges associated with DOE Oak Ridge operations (Table 4.1). Fish from

PCK 10.4 contained mercury concentrations typical of fish from reference streams (Table 4.1), indicating that neither upstream sources of mercury in Poplar Creck nor movement of fish between sites were probable explanations for the high mercury levels at PCKs 8.2 and 6.9, located just upstream (PCK 8.2) and downstream (PCK 6.9) of Mitchell Branch. Although no fish exceeded the FDA action level of $1 \mu \mathrm{g} / \mathrm{g}, 25$ of 56 fish collected from PCKs 8.2 and 6.9 exceeded the more conservative PGV of $0.42 \mu \mathrm{g} / \mathrm{g}$ (Travis et al. 1986). Mean mercury concentrations in fish from these two sites changed little between 1987 and 1990; mean values were about $0.4 \mu \mathrm{g} / \mathrm{g}$ at both sites in most years. Furthermore, no significant difference (ANOVA) in mean mercury concentrations was evident between PCKs 8.2 and 6.9 (0.40 and $0.38 \mu \mathrm{g} / \mathrm{g}$, respectively), suggesting that Mitchell Branch was not a significant additional contributor of mercury to fish in Poplar Creek between 1987 and 1990.

The high mercury levels in fish from PCKs 8.2 and 6.9 are not readily explainable by a one-source-hypothesis, such as input from EFPC only. Levels of mercury in sunfish from EFPC decreased in propor- 


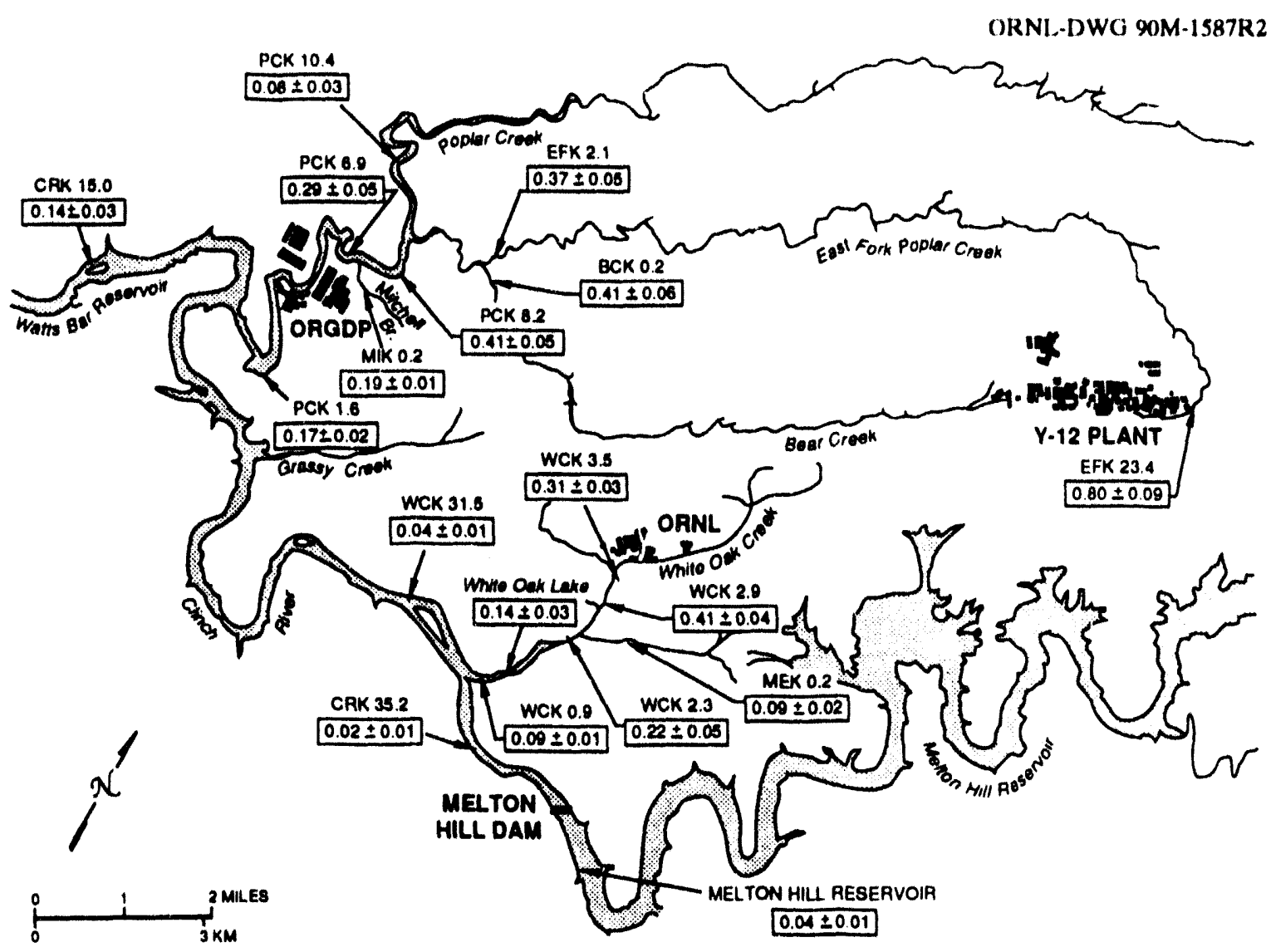

Fig. 4.2. Mean concentrations ( \pm 1 SE) of mercury (in micrograms per gram, wet weight) in bluegill $(n=8$ ) collected in fall/winter 1988 at sites on the Oak Ridge Reservation and nearby reaches of the Clinch River. Rockbass (Ambloplites rupestris) and redbreast sunfish (Lepomis auritus) were substituted for bluegill at Bear Creek kilometer 0.2 and Mitchell Branch kilometer 0.2 respectively.

tion to dilution of Oak Ridge Y-12 Plant discharges at sites along the length of EFPC (G. R. Southworth, ESD/ORNL, unpublished data), yet mercury levels in fish from PCKs 8.2 and 6.9 were similar to those found in lower EFPC, despite an almost fourfold dilution of EFPC upon entering Poplar Creek. One potential source of this mercury is Bear Creek. Mercury contamination is evident in fish from lower Bear Creek, but the extent of contamination in fish does not appear to be large enough to explain the high levels seen in Poplar Creek fish (Figs. 4.1 and 4.2). It is possible that either changes occur in the environmental chemistry of mercury in lower Poplar Creek, enhancing the biological availability of water-borne or sediment-associated mercury, or that additional sources of mercury occur in the reach of Poplar Creek near the Oak Ridge K-25 Site.

Mercury contamination in fish at the most downstream site on Poplar Creek (PCK 1.6) was much lower than at PCKs 6.9 or 8.2. Similarly low concentrations were found in fish in the Clinch River (CRK 15.0) downstream of the mouth of Poplar Creek. Mercury concentrations in fish at CRK 15.0 were slightly elevated over concentrations in fish at sites on the Clinch River upstream of the 
mouth of Poplar Creek; however, these levels were very similar to those found in fish from a reference stream (Hinds Creek). Thus, mercury contamination from Oak Ridge Operations appears to be detectable in Clinch River fish downstream of the mouth of Poplar Creek, but the degree of contamination appears to be minimal.

The degree of mercury contamination in bluegill from Poplar Creek sites near the K-25 Site was generally low compared with the degree of contamination in fish from the middle to upper reaches of EFPC in 1987 and 1988 (Loar et al. 1993b). Mean $\mathrm{Hg}$ concentrations at PCKs 8.2 and 6.9 ranged from approximately one-fourth to one-half the mercury concentrations in bluegill at EFK 23.4 (Figs. 4.1 and 4.2). In general, the mean $\mathrm{Hg}$ concentrations in fish at these two sites were comparable with those from lowermost EFPC (EFK 2.1) and Bear Creek (BCK 0.2) and the worst mercury-contaminated sites on White Oak Creek (WCKs 3.5 and 2.9). Mean mercury concentrations in fish from lowermost Poplar Creek (PCK 1.6) and the Clinch River (CRK 15.0) were comparable with those of fish from downstream sites on White Oak Creek. Fish from Mitchell Branch had $\mathrm{Hg}$ concentrations similar to those in fish from PCK 1.6, CRK 15.0, and lower White Oak Creek sites (WCKs 1.0 and 2.3; Loar 1989). However, the Mitchell Branch fish are substantially smaller than those from PCK 1.6, CRK 15.0, and WCK 2.9. As discussed, mean mercury concentrations in Mitchell Branch fish would probably be much higher if more adult fish were present.

\subsubsection{Other Metals in Mitchell Branch Fish}

Despite abnormally high levels of some metals in Mitchell Branch water (Smith et al. 1993) and sediment (Ashwood et al. 1986), concentrations of metals (other than mercury) in Mitchell Branch fish in all three sampling years were similar to those found in the reference stream (Table 4.2). Although cadmium and copper concentrations in Mitchell Branch fish in 1987 were slightly higher than in Hinds Creek fish collected in that year (Smith et al. 1993), the difference was not statistically significant. The most recent sampling of Mitchell Branch (1990) revealed that only two metals other than $\mathrm{Hg}$ (Se and $\mathrm{Zn}$ ) were found above the analytical detection limit. The levels of metals (except for mercury) in Mitchell Branch fish in all three sampling years were also quite similar to those observed in Hinds Creek bluegill in 1987 (Smith et al. 1993) and by the Tennessee Valley Authority (TVA) at one of their reference sites (Melton Hill Reservoir) (TVA 1985, 1986). Moreover, the levels of metals observed in fish in the National Contaminant Biomonitoring Program (geometric mean of 112 sites sampled for $\mathrm{As}, \mathrm{Cd}, \mathrm{Cu}$, $\mathrm{Pb}, \mathrm{Hg}$, Se, and $\mathrm{Zn}$; Lowe et al. 1985) were also generally similar to levels observed in Mitchell Branch fish, except for mercury.

A comparison of the concentrations of metals in Mitchell Branch fish with PGVs, derived to screen for levels of contami-nation that may potentially threaten human health (Travis et al. 1986; Hoffman et al. 1984), indicates that only $\mathrm{As}, \mathrm{Be}$, and $\mathrm{Hg}$ approach this threshold (Table 4.2). Beryllium and arsenic were also the only two metals in Mitchell Branch fish that exceeded fish tissue screening levels established for protection of human health by the EPA (EPA 1990). Neither arsenic nor beryllium was detected in Mitchell Branch fish; however, PGV and EPA screening levels are set at levels below current detection limits because of the carcinogenicity of these two metals. The PGV screening approach is very conservative and is designed to eliminate 
Table 4.2 Metal concentrations (mean $\pm 1 \mathrm{SE}$, in micrograms per gram wet weight) in redbreast sunfish (Lepomis auritus) collected from Mitchell Branch and Hinds Creek (reference stream)

\begin{tabular}{|c|c|c|c|c|c|}
\hline \multirow[b]{2}{*}{ Metal } & \multicolumn{3}{|c|}{ Mitchell Branch } & \multirow[b]{2}{*}{ Hinds Creek } & \multirow[b]{2}{*}{$P G V^{c}$} \\
\hline & $1987^{a}$ & 1988 & 1990 & & \\
\hline Antimony & $<0.2$ & $<0.3$ & $<0.54$ & $<0.44$ & 5.2 \\
\hline Arsenic & $<0.10$ & $<0.3$ & $<0.05$ & $<0.3$ & 0.0007 \\
\hline Beryllium & $<0.05$ & $<0.3$ & $<0.003$ & $<0.3$ & 0.004 \\
\hline Cadmium & $0.05 \pm 0.01$ & $<0.005$ & $<0.22$ & $<0.18$ & 1.0 \\
\hline Chromium & $<0.1$ & $<0.5$ & $<0.54$ & $<0.5$ & 1.8 \\
\hline Copper & $0.68 \pm 0.14$ & $0.09 \pm 0.03$ & $<0.54$ & $0.32 \pm 0.13$ & 36 \\
\hline Lithium & $<0.5$ & $<0.3$ & & $<0.3$ & \\
\hline Mercury & $0.17 \pm 0.01^{d}$ & $0.12 \pm 0.01^{e}$ & $0.27 \pm 0.03$ & $0.08 \pm 0.01^{f}$ & 0.42 \\
\hline Nickel & $<1.0$ & $<1.0$ & $<0.54$ & $<1.0$ & 5.2 \\
\hline Lead & $0.08 \pm 0.05$ & $<0.1$ & $<0.54$ & $<0.44$ & 1.8 \\
\hline Selenium & $0.50 \pm 0.10$ & $0.40 \pm 0.04$ & $0.61 \pm 0.03$ & $0.52 \pm 0.08$ & 12 \\
\hline Silver & $<0.05$ & $<0.05$ & $<0.22$ & $<0.18$ & 0.29 \\
\hline Thallium & $<0.2$ & $<0.2$ & $<0.02$ & $<0.2$ & 0.66 \\
\hline Zinc & $5.5 \pm 0.3$ & $1.8 \pm 0.5$ & $4.7 \pm 0.3$ & $5.0 \pm 0.5$ & 180 \\
\hline
\end{tabular}

$a_{n}=6$ except for mercury.

bHinds Creek reference fish for metals other than mercury were collected in December of 1988 and $1990(n=2)$.

cPreliminary Guidance Values (C. C. Travis et al. 1986, Preliminary review of TVA fish sampling and analysis report, Report of Task Group Five to Oak Ridge Task Force, Oak Ridge National Laboratory, Oak Ridge, Tenn., Mimeo, Jan. 1986. F. O. Hoffman et al. 1984, Preliminary screening of contaminants in sediments, ORNL/TM-9730, Oak Ridge National Laboratory, Oak Ridge, Tenn.).

${ }^{d} n=7$.

$n=5$.

Fish collected from 1985 to $1989(n=46)$.

Note: Mitchell Branch fish were collected in May 1987, March 1988, and January 1990 ( $n=4$ except where noted). W'hen all values used to calculate the mean were below detection limits, the highest detection limit value was cited. 
from concern any substances not exceeding the PGV (Hoffman et al. 1984).

\subsubsection{PCBs in Mitchell Branch Fish}

Results presented previously for Mitchell Branch (Smith et al. 1993) revealed that Asiatic clams held for 4-week exposures in cages accumulated substantial concentrations of PCBs. It was hypothesized that if adequate size and numbers of resident fish were present they would contain substantial PCB concentrations as well. Results of PCB analysis of redbreast collected in March 1989 and January 1990 confirmed this hypothesis (Table 4.3). Mitchell Branch fish contained significantly higher mean PCB concentrations than reference stream fish in both years $(p<0.05, t$-test $)$. The level of PCB contamination in fish (mean of $1.18 \mu \mathrm{g} / \mathrm{g}$, both years combined) is high for a relatively short-lived, low-lipid species such as redbreast sunfish; longer-lived older, fattier species such as channel catfish, carp (Cyprinus carpio), or largemouth bass (Micropterus salmoides), if present, would probably contain much higher levels. In general, the degree of PCB contamination in redbreast from Mitchell Branch was similar to the level of contamination found at the most highly contaminated sites on East Fork Poplar Creek (EFK 23.4) and White Oak Creek (WCK 2.9) (Figs. 4.3 and 4.4). PCB levels in Mitchell Branch fish could pose concern for human exposure; two of eight redbreast in 1989 contained PCB concentrations above the FDA action level (FDA 1984b).

The significance of Mitchell Branch as a source of PCB contamination is evident when comparing the levels of contamination in fish collected in Poplar Creek upstream and downstream of the mouth of Mitchell Branch. Because no statistically significant relationship was found between $\mathrm{PCB}$ concentrations in fish tissue and fish weight (ANOVA; $p>0.05$ ), comparisons were made among sites without adjusting for fish size. Additionally, no adjustments were made when mean PCB concentrations were compared between redbreast sunfish and bluegill from different sites, because past studies on the reservation (Loar 1992b; Loar 1993a) have shown that mean PCB concentrations between redbreast and bluegill collected from the same sites were not significantly different. Mean PCB concentrations in Mitchell Branch redbreast were significantly higher $(p<0.05)$ than mean PCB concentrations in bluegill at PCKs 6.9 and 8.2 in each year (Table 4.3). Much higher concentrations were found in fish from Mitchell Branch than in fish from PCK 6.9, even though PCK 6.9 is only a short distance downstream of Mitchell Branch. These results confirm the conclusions drawn from the earlier clam data (i.e., Mitchell Branch is a substantial source of PCBs to its own biota; fish were not likely to have obtained such high PCB concentrations elsewhere).

\subsubsection{PCBs in Poplar Creek Fish}

PCB concentrations were measured in bluegill from several sites in Poplar Creek and the Clinch River to assess the relative importance of various streams (Bear Creek, EFPC, Mitchell Branch, and White Oak Creek) to the system. PCB results for the Poplar Creek area are shown in Table 4.3; results for the entire reservation are shown in Figs. 4.3 and 4.4.

Fish from PCK 10.4, upstream of any discharges associated with DOE Oak Ridge operations, contained PCB concentrations typical of reference stream fish (Table 4.3), indicating that neither upstream sources of PCBs in Poplar Creek nor movement of fish between sites was a probable explanation for the high PCB levels at PCKs 8.2 and 6.9. As was observed for mercury, the mean concentrations of PCBs in fish from 
Table 4.3. PCB concentrations in bluegill (Lepomis macrochirus), redbreast sunfish (L. auritus), channel catish (Ictalurus punctatus), and girzard shad (Dorosoma cepedianum) collected at sites in the Poplar Creek drainage area in the vicinity of the Oak Ridge K-25 Site

\begin{tabular}{|c|c|c|c|c|c|}
\hline Site & Species & Date & $\begin{array}{c}\text { PCB } \\
\text { mean } \pm 1 \text { SE } \\
(\mu g / g, \text { wet } w t)\end{array}$ & Range & $\begin{array}{c}\text { FDA } \\
\text { exceedences }\end{array}$ \\
\hline EFK 2.1 & Bluegill & $\begin{array}{l}\text { December } 1987 \\
\text { November } 1988\end{array}$ & $\begin{array}{l}0.14 \pm 0.04 \\
0.13 \pm 0.03\end{array}$ & $\begin{array}{l}0.03-0.41 \\
0.03-0.33\end{array}$ & $\begin{array}{l}0 / 8 \\
0 / 8\end{array}$ \\
\hline PCK 10.4 & Bluegill & $\begin{array}{l}\text { November } 1987 \\
\text { November } 1988\end{array}$ & $\begin{array}{l}0.06 \pm 0.01 \\
0.05 \pm 0.03\end{array}$ & $\begin{array}{l}0.02-0.11 \\
0.01-0.26\end{array}$ & $\begin{array}{l}0 / 8 \\
0 / 8\end{array}$ \\
\hline PCK 8.2 & Bluegill & $\begin{array}{l}\text { November } 1987 \\
\text { November } 1988 \\
\text { March } 1990\end{array}$ & $\begin{array}{l}0.20 \pm 0.03 \\
0.31 \pm 0.07 \\
0.07 \pm 0.02\end{array}$ & $\begin{array}{l}0.11-0.36 \\
0.08-0.59 \\
0.02-0.15\end{array}$ & $\begin{array}{l}0 / 8 \\
0 / 8 \\
0 / 8\end{array}$ \\
\hline MIK 0.2 & Redbreast & $\begin{array}{l}\text { March } 1989 \\
\text { January } 1990\end{array}$ & $\begin{array}{l}1.61 \pm 0.32 \\
0.80 \pm 0.14\end{array}$ & $\begin{array}{l}0.50-3.27 \\
0.23-1.55\end{array}$ & $\begin{array}{l}2 / 8 \\
0 / 8\end{array}$ \\
\hline \multirow[t]{3}{*}{ PCK 6.9} & Bluegill & $\begin{array}{l}\text { June } 1987 \\
\text { November } 1987 \\
\text { November } 1988 \\
\text { March } 1990\end{array}$ & $\begin{array}{l}0.22 \pm 0.06 \\
0.26 \pm 0.04 \\
0.21 \pm 0.05 \\
0.12 \pm 0.01\end{array}$ & $\begin{array}{l}0.05-0.53 \\
0.14-0.47 \\
0.08-0.40 \\
0.07-0.23\end{array}$ & $\begin{array}{l}0 / 8 \\
0 / 8 \\
0 / 7 \\
0 / 8\end{array}$ \\
\hline & Channel catfish & $\begin{array}{l}\text { August } 1988 \\
\text { August } 1989\end{array}$ & $\begin{array}{l}0.71 \pm 0.13 \\
1.07 \pm 0.35\end{array}$ & $\begin{array}{l}0.28-1.31 \\
0.25-3.37\end{array}$ & $\begin{array}{l}0 / 8 \\
1 / 8\end{array}$ \\
\hline & Gizzard shade & August 1988 & $2.97 \pm 0.76$ & $1.76-4.37$ & \\
\hline PCK 1.6 & Bluegill & $\begin{array}{l}\text { December } 1987 \\
\text { November } 1988\end{array}$ & $\begin{array}{l}0.17 \pm 0.03 \\
0.19 \pm 0.04\end{array}$ & $\begin{array}{l}0.10-0.37 \\
0.06-0.39\end{array}$ & $\begin{array}{l}0 / 8 \\
0 / 8\end{array}$ \\
\hline \multirow[t]{3}{*}{ CRK 15.0} & Bluegill & $\begin{array}{l}\text { November } 1987 \\
\text { November } 1988\end{array}$ & $\begin{array}{l}0.08 \pm 0.01 \\
0.11 \pm 0.03\end{array}$ & $\begin{array}{l}0.04-0.15 \\
0.01-0.28\end{array}$ & $\begin{array}{l}0 / 8 \\
0 / 8\end{array}$ \\
\hline & Channel catfish & $\begin{array}{l}\text { August } 1988 \\
\text { August } 1989\end{array}$ & $\begin{array}{l}0.50 \pm 0.07 \\
0.79 \pm 0.25\end{array}$ & $\begin{array}{l}0.15-0.90 \\
0.01-2.10\end{array}$ & $\begin{array}{l}0 / 8 \\
1 / 8\end{array}$ \\
\hline & Gizzard shad ${ }^{c}$ & August 1988 & $0.82 \pm 0.12$ & $0.58-0.94$ & \\
\hline \multicolumn{6}{|c|}{ Reference sites } \\
\hline MHR & Bluegill & $1987-1988$ & $0.09 \pm 0.02$ & $0.02-0.28$ & $0 / 16$ \\
\hline & $\begin{array}{l}\text { Channel catfish } \\
\text { Gizzard shad" }\end{array}$ & $\begin{array}{l}\text { 1988-1989 } \\
\text { August } 1988\end{array}$ & $\begin{array}{l}0.41 \pm 0.09 \\
0.40 \pm 0.03\end{array}$ & $\begin{array}{l}0.07-1.61 \\
0.35-0.44\end{array}$ & $\begin{array}{l}0 / 18 \\
0 / 8\end{array}$ \\
\hline $\mathrm{HC}$ & Redbreast & $1985-1989$ & $0.04 \pm 0.01$ & $0.00-0.18$ & $0 / 50$ \\
\hline
\end{tabular}

${ }^{\top}$ MHR $=$ Melton Hill Reservoir; HC $=$ Hinds Creek; EFK = East Fork Poplar Creek kilometer; PCK $=$ Poplar Creek kilometer, MIK = Mitchell Branch kilometer, $\mathrm{CRK}=$ Clinch River kilometer.

'Number of samples exceeding the FDA action level of $2.00 \mu \mathrm{g} / \mathrm{g}$ [U.S. Department of Agriculture Food and Drug Administration 1984, Polychlorinated biphenyls (PCBs) in fish and shellfish: Reduction of Tolerances, Final Decision, Fed. Regist. 49.(100):21520] divided by the total number of samples.

The mean and range for gizzard shad represents three composite sam, of five shad each. 
RNL-DWG 88-15329

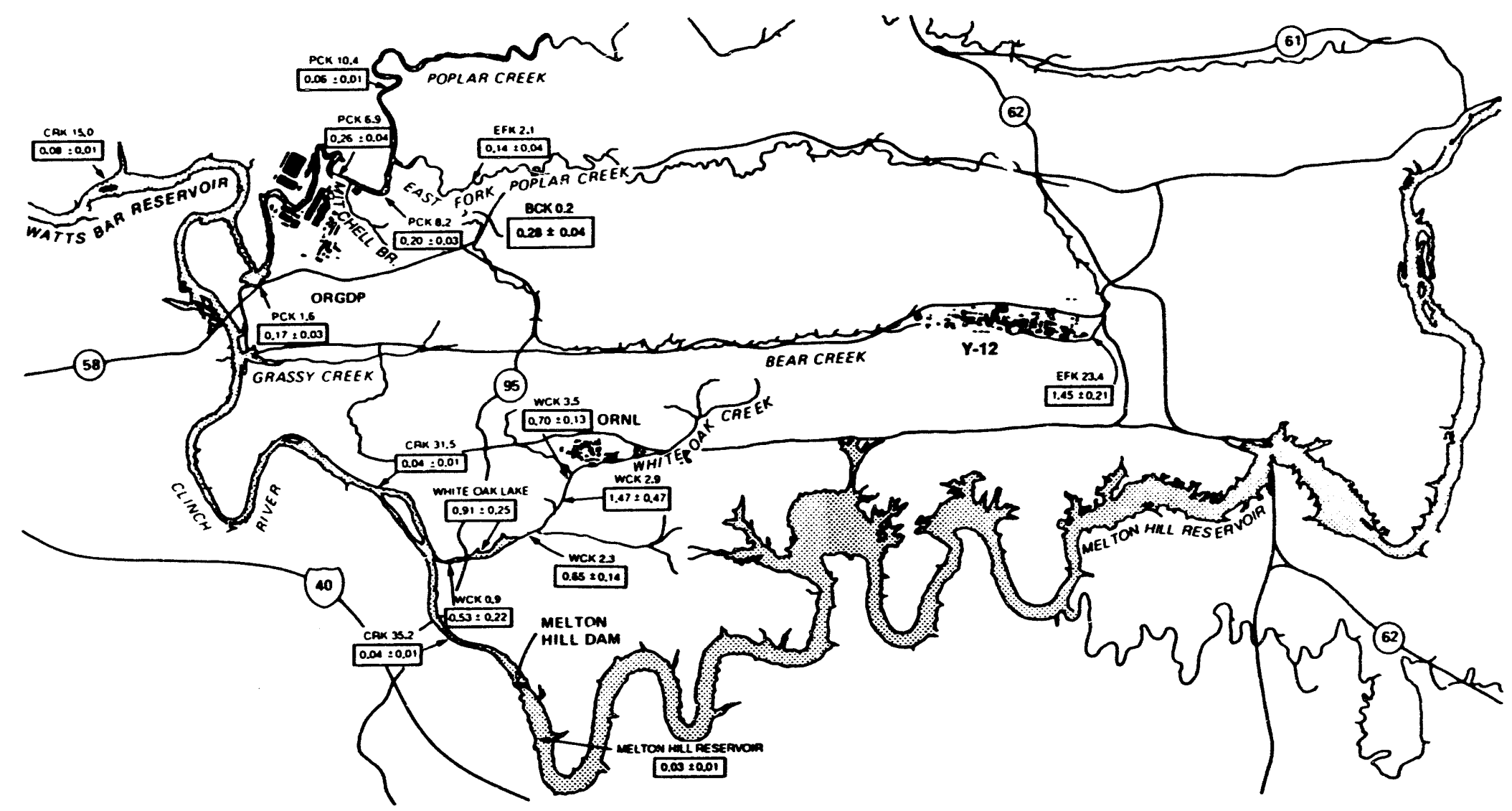

Fig. 4.3. Mean concentrations $( \pm 1 \mathrm{SE}$ ) of poiychlorinated biphenyl (in micrograms per gram, wet weight) in bluegill $(n=8)$ collected in fall/winter 1987 at sites on the Oak Ridge Reservation and nearby reaches of the Clinch River. Rockbass (Ambloplites rupestris) and redbreast sunfish (Lepomis auritus) were substituted for bluegill at Bear Creek kilometer 0.2. 


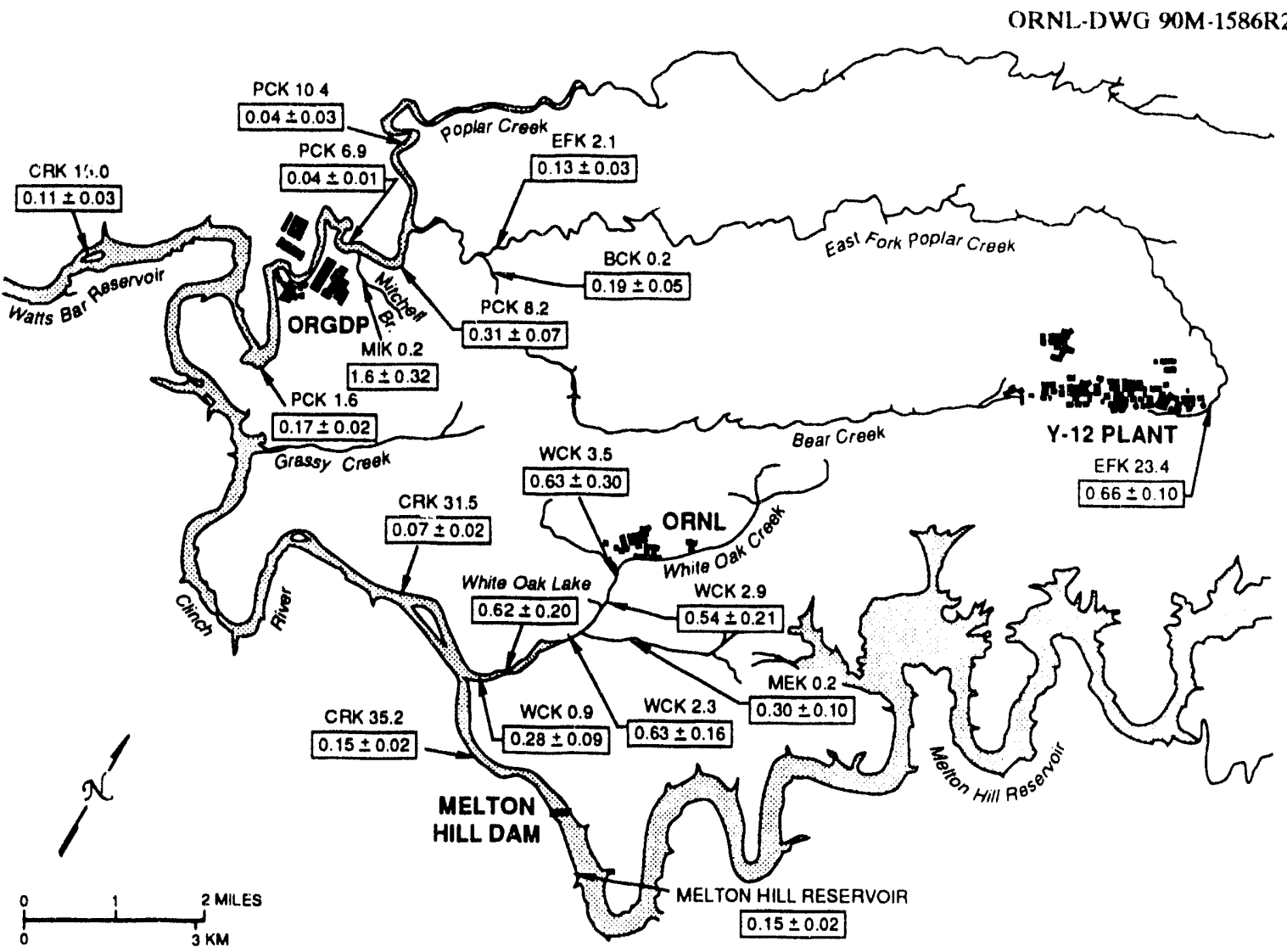

Fig. 4.4. Mean concentrations $( \pm 1$ SE) of polychlorinated biohenyl (in micrograms per gram, wet weight) in bluegill $(n=8)$ collected in fall/winter 1988 at sites on the Oak Ridge Reservation and neisby reaches of the Clinch River. Rockbass (Ambloplites rupestris) and redbreast sunfish (Lepomis auritus) were substituted for bluegill at Bear Creek kilometer 0.2 and Mitchell Branch kilometer 0.2 respectively.

lower Poplar Creek (PCKs 8.2, 6.9, and 1.6) did not decline proportionally to the dilution of EFPC in Poplar Creek, but rather remained at levels typical or higher than those of lower Ei'PC (Figs. 4.3 and 4.4). In 1987 and 1988, the mean PCB concentration in bluegill collected downstream of Mitchell Branch (PCK 6.9) was not significantly different from that for bluegill at the site upstream of Mitchell Branch (PCK 8.2). In 1990, the mean PCB concentration in bluegill at PCK 6.9 was higher than at PCK 8.2, but concentrations at both sites were at or near background. Tha relatively low levels of PCBs in bluegill at PCK 6.9 (mean of
0.22 , all years), coupled with the lack of substantial increases in PCBs at PCK 6.9 compared with PCK 8.2, suggest that PCB inputs from Mitchell Branch have little impact on Poplar Creek. However, the use of sunfish to monitor very dilute inputs of PCBs may be limited because lipid-rich species collected at PCK 6.9 (channel catfish and gizzard shad) contained much higher levels of PCBs (Table 4.3).

The degree of $\mathrm{PCB}$ contamination in bluegill from the Poplar Creek sites near the Oak Ridge K-25 Site (PCKs 8.2, 6.9, and 1.6) was relatively low compared with other sites on the reservation (Figs. 4.3 and 4.4). The level of PCB contamination 
in Poplar Creek bluegill was most similar to levels in fish from lowermost EFPC, in rockbass from Bear Creek (Loar 1993b), and in fish from the lowermost part of the White Oak Creek drainage [White Oak Lake and White Oak Creek kilometer (WCK) 0.2].

PCB contamination in Poplar Creek appeared to have little or no impact on PCB concentrations in bluegill in the Clinch River (Table 4.3). Mean PCB concentrations in bluegill from the Clinch River downstream of the mouth of Poplar Creek (CRK 15.0) were slightly elevated over upstream sites on the Clinch River [CRKs 31.5 and 35.5 and Melton Hill Reservoir (MHR)] in 1987, but in 1988 concentrations in fish at CRK 15.0 were at or below PCB levels upstream (Figs. 4.3 and 4.4).

The Poplar Creek embayment continues to receive ongoing $\mathrm{PCB}$ inputs from at least three sources: EFPC, Bear Creek, and Mitchell Branch. Resolving the significance of each PCB source on the PCB burden of Poplar Creek biota is not possible with the existing data. Further complicating an evaluation of possible sources to Poplar Creek is the fact that Poplar Creek is a depositional backwater of Watts Bar Reservoir and thus is likely to accumulate PCB-contaminated sediments. Some of the observed contamination in fish may be a result of mobilization of sediment associated PCBs rather than ongoing stream discharges.

Channel catfish were collected at PCK 6.9 and CRK 15.0 in 1988 and 1989 as part of an evaluation of the importance of inputs from DOE sources in contributing to PCB contamination in Watts Bar Reservoir. Results of this study are given in detail in Loar (1993a) and are only summarized here. Mean PCB concentrations in channel catfish from PCK 6.9 in both years were elevated over concentrations in catfish at both the downstream site on the Clinch River (CRK 15.0) and
MHR (Table 4.3). However, these differences were not great, considering the discrete nature of EFPC/Poplar Creek as a source of PCB contamination. It is likely that movements of channel catfish and forage fish act to spread PCB contamination away from point sources, which results in a rather uniform distribution of PCB contamination in catfish throughout Watts Bar Reservoir, as was observed in the Oak Ridge Task Force study in 1984 (TVA 1985). One in eight fish collected in 1989 from both CRK 15.0 and PCK 6.9 exceeded the 2-ppm action level. The mean PCB concentrations in channel catfish in 1988 and 1989 at PCK 6.9 support the precautionary fish consumption advisory issued by the Tennessee Department of Health and Environment.

Gizzard shad were collected concurrently with catfish in 1988 to assess the significance of PCB accumulation in forage fish. The mean PCB concentration of three composite samples (five gizzard shad each) collected at PCK 6.9 was significantly higher than for shad collected from CRK 15.0 and MHR $(p<0.05)$ (Loar 1993a). The PCB concentration of nearly $3 \mathrm{ppm}$ in shad from PCK 6.9 clearly indicates that Poplar Creek embayment, like White Oak Creek embayment (Loar 1993a), is a relative " $r_{\mathrm{o}}$ ot spot" for PCB contamination. Highly contaminated gizzard shad from the area are likely to be important vectors for PCB dispersal to larger game fish that feed on shad (e.g., largemouth bass, catfish, and rockfish).

The mean PCB concentration in shad from CRK 15.0, as the case with bluegill and channel catfish, was not significantly different from the concentrations in shad from upstream sites (CRK 32.2 and MHR) (Loar 1993a). Evaluating the effect of various PCB inputs from DOE facilities on fish in the Clinch River proper is complicated by two factors: (1) substantial PCB contamination is evident in fish immediately upstream of any DOE impacts 
(MHR) and (2) the species that tend to accumulate the highest levels of PCBs (catfish and shad) appear to range widely, obscuring relationships between source location and PCB levels in fish.

\subsubsection{PCBs in Caged Clams}

Caged clams held in Mitchell Branch for four weeks accumulated substantial concentrations of PCBs in all exposure periods from 1988 to 1990 (Table 4.4). Mean concentrations ranged from $0.34 \mu \mathrm{g} / \mathrm{g}$ in April 1988 to $0.98 \mu \mathrm{g} / \mathrm{g}$ in July 1989. Previous clam exposures in Mitchell Branch also resulted in substantial PCB accumulation (Smith et al. 1993). The degree of PCB accumulation noted in clams was consistent with the relatively high concentrations of PCBs found in redbreast and bluegill sunfish from Mitchell Branch in 1989 and 1990 (Table 4.3).

PCB concentrations in clams in 1988-90 were lower than those observed in 1987, when clams were exposed soon after a PCB discharge in the Mitchell Branch drainage (Smith et al. 1993). However, there does not appear to have been a continuing decrease in biotic PCB contamination over the 1988-90 period. In fact, the highest PCB concentrations observed in 1988-90 occurred in July 1989 and April 1990 (Table 4.4). Interpretation of temporal trends in the degree of PCB contamination must be made with cautiori because adverse environmental conditions, such as lack of food or the presence of toxicants, may result in atypically low PCB accumulation by clams despite the presence of PCB contamination. Thus, it may be possible under improving ecological conditions in a highly affected stream, such as Mitchell Branch, for PCB accumulation in saged clams to increase over time without a concurrent increase in PCB inputs to the system. The data collected to date show that Mitchell Branch contains significant
PCB contamination, which is not rapidly increasing or decreasing.

In 1988, clams placed downstream

from the mouth of Mitchell Branch accumulated slightly higher concentra* :ons of PCBs than clams placed upstream from Mitchell Branch (Table 4.4). These data suggest that the impact of PCB discharges from Mitchell Branch to Poplar Creek may be discernable in Poplar Creek biota, although no definite conclusions can be drawn from single sampling. As noted in Sect. 4.3.5, such an impact is not apparent in Poplar Creek sunfish.

The concentrations of PCBs measured in caged clams in Mitchell Branch indicate that this stream is among the most highly PCB contaminated aquatic sites on ORR (Table 4.5). Levels of PCB contamination in clams from Mitchell Branch in 1988-90 were similar to those measured in clams from White Oak Creek/White Oak Lake, Bear Creek, and EFPC immediately downstream from the Oak Ridge Y-12 Plant. PCB concentrations in resident sunfish at these sites (Table 4.3) are similar to those observed in caged clams following a 4-week exposure.

\subsubsection{Other Organics in Caged Clams}

Some chemicals, such as polycyclic aromatic hydrocarbons (PAHs) do not accumulate appreciably in fish because of their rapid conversion into more soluble metabolites. Because clams such as Corbicula have less developed capabilities for detoxifying organic contaminants than fish, these chemicals can accumulate substantially in clams if they are present in solution or in particles filtered while feeding. Analysis of clams for semivolatile organic compounds and PAHs did not reveal any substances not also found at similar concentrations in reference stream clams, except for the detection of very low concentrations of PAHs in 1990 
Table 4.4. Concentrations of polychlorinated biphenyls (PCBs) and other organic contaminants in caged clams (Corbicula fluminea) maintained in

Mitchell Branch for 4 weeks

\begin{tabular}{|c|c|c|c|}
\hline Site & Date & $\begin{array}{c}\mathrm{PCB}^{a} \\
(\mu \mathrm{g} / \mathrm{g} \text { wet } w t)\end{array}$ & $\begin{array}{l}\text { Other organics }{ }^{a} \\
(\mu g / g \text { wet } w t)\end{array}$ \\
\hline MIK 0.2 & April 1988 & $0.34 \pm 0.07$ & $\mathrm{BLD}^{b, c}$ (below limit of detection) \\
\hline MIK 0.2 & April 1989 & $0.69 \pm \underset{(2)}{ \pm} 0.02$ & \\
\hline MIK 0.2 & July 1989 & $0.98 \pm 0.10$ & $\mathrm{BLD}^{d}$ \\
\hline MIK 0.2 & April 1990 & $0.91 \underset{(2)}{ \pm} 0.06$ & $\begin{array}{l}\text { aldrin, } 0.045 \\
\text { chlordane }, 0.007 \\
\text { acenaphthene }, 0.008 \\
\text { anthracene }, 0.001 \\
\text { benz(a)anthracene', } 0.009 \\
\text { chrysene', } 0.004 \\
\text { fluorene', } 0.022 \\
\text { Others BLD }\end{array}$ \\
\hline PCK 8.2 & April 1988 & $0.12 \pm \underset{(3)}{0.00}$ & \\
\hline PCK 6.9 & Apriı 1988 & $0.19 \pm 0.01$ & \\
\hline \multirow[t]{4}{*}{ Reference sites } & April 1988 & $\begin{array}{c}0.05 \pm 0.00 \\
(3)\end{array}$ & $B L D^{b, c}$ \\
\hline & April 1989 & $0.12 \pm 0.01$ & \\
\hline & July 1989 & $<0.02$ & BLD $^{d}$ \\
\hline & April 1990 & $<0.09$ & BLD $^{\prime}$ \\
\hline
\end{tabular}

"Values are mean $\pm 1 \mathrm{SE}$, with the number of samples in parentheses.

${ }^{b} \mathrm{Bis}$ (2-ethylhexyl)phthalate, diethlphthalate, benzoic acid, and bis(2-chloroisopropyl)ether were detected in reference site samples and MIK samples.

'Diethylphthalate and benzoic acid were detected in reference site samples and MIK samples.

Diethylphthalate was detected in reference site samples and MIK samples.

These trace concentrations of these substances were reported at levels far below the analytical limit of quantification, but at higher concentrations than found in reference site samples. Confirmation that these substances were indeed present is impossible at such low levels.

Diethylphthalate and dibutylphthalate were detected in reference site and MIK samples.

Note: MIK $=$ Mitchell Branch kilometer; PCK $=$ Poplar Creek kilometer. 
Table 4.5. Range of mean PCB concentrations

measured in caged clams (Corbicula

flumine d) at PCB-contaminated

$s$ tes on Oak Ridge

Reservation,

1988-90

\begin{tabular}{lc}
\hline \multicolumn{1}{c}{ Site } & $\begin{array}{c}\text { PCBs } \\
(\mu \mathrm{g} / \mathrm{g}, \text { wet wt })\end{array}$ \\
\hline Mitchell Branch, km 0.2 & $0.34-0.98$ \\
Bear Creek, km 4.5 & $0.32-1.01$ \\
East Fork Poplar Creek, km 23.4 & $0.18-0.47$ \\
White Oak Creek, km 2.6 & $0.23-0.89$ \\
White Oak Lake & $0.41-0.83$ \\
\hline
\end{tabular}

(Table 4.4). Improvements in analytical detection limits in 1990 enabled the detection of lower concentrations of PAHs than was possible in previous years. Concentrations of several PAHs were clearly higher in clams held in Mitchell Branch than in clams at the reference sites; however, the concentrations were very low, the highest being fluorene $(0.022 \mu \mathrm{g} / \mathrm{g})$. Given the presence of coal pile runoff and hydrocarbon contamination in Mitchell Branch, it is not surprising to find traces of PAHs in clams. This finding corroborates the detection of elevated levels of mixed function oxidases in fish in Mitchell Branch (Sect. 5.3.1) because PAHs stimulate the production of such detoxification enzymes (Payne and Penrose 1975).

Analysis of clams for hydrophobic pesticides detected aldrin in one of two clam samples at a concentration of $0.04 \mu \mathrm{g} / \mathrm{g}$. This agricultural pesticide is no longer sold in the United Stcies, and its occurrence at the Oak Ridge K-25 Site is inexplicable. Similar concentrations of aldrin were reported in one clam sample from Bear Creek and in a reference site clam sample that had been spiked with PCBs, PAH, and phthalates to determine percentage recovery of analytes through the analytical procedure. Aldrin was not present in the spike, nor was it detected in the unspiked reference site clams. Thus, it appears likely that the aldrin reported in one Mitchell Branch clam sample was a result of a positive interference in the analytical procedure. Annual monitoring for pesticide contamination will continue in Mitchell Branch; if aldrin contamination is present, it should appear in future samples.

\subsection{CONCLUSIONS}

Mercury and PCBs are the two primary contaminants that accumulate above background levels in Mitchell Branch biota. From 1987 through 1990, the levels of mercury were not excessive relative to the FDA limit and were similar to those in fish in nearby Poplar Creek. However, the significance of Mitchell Branch as a source of the elevated $\mathrm{Hg}$ levels observed in its fish, or in fish from lower Poplar Creek, remains unclear. Elevated concentrations of other metals were not found in fish from Mitchell Branch. 
The PCB monitoring data were more conclusive and clearly showed that Mitchell Branch was a source of PCB contamination to its biota. Some fish in Mitchell Branch contained PCB concentrations in excess of the $2 \mu \mathrm{g} / \mathrm{g}$ FDA action level. Significant PCB contamination $(>1 \mu \mathrm{g} / \mathrm{g})$ was also evident in channel catfish and gizzard shad collected in Poplar Creek a short distance downstream of Mitchell Branch. Mitchell Branch undoubtedly is a source of PCB contamination to Poplar Creek, but its significance as a source of PCB contamination to the biota in the Clinch River arm of Watts Bar Reservoir relative to other possible sources (EFPC, White Oak Creek, Bear Creek, and Poplar Creek sediment), cannot be ascertained from these data. Caged clams placed in Mitchell Branch accumulated very low concentrations of PAHs and substantial concentrations of PCBs. Oil and coal inputs to the stream probably account for the trace amounts of PAHs, which in turn are probably the causes of the elevated concentrations of detoxification enzymes in fish reported in Sect. 5.3.1. A discontinued agricultural insecticide, aldrin, was detected in one of two clam samples analyzed in 1990, but its detection in two other unrelated samples suggest that it may be the result of an analytical interference.

\subsection{FUTURE STUDIES}

The most significant contaminant bioaccumulation problem in Mitchell Branch appears to be PCBs. A key objective of future studies will be to identify and characterize the PCB source(s) in Mitchell Branch (e.g., specific ongoing discharges, sediment contamination, and episodic releases). Placement of clam cages further upstream near possible PCB discharges, along with sediment sampling of Mitchell Branch may help in locating the PCB source(s).

Resident fish and caged clams will continue to be used for monitoring the accumulation of metals and organics in Mitchell Branch. Bluegill will continue to be collected from sites on Poplar Creek to further evaluate the role of Mitchell Branch as a source of mercury and PCBs in that system. To evaluate the significance of Mitchell Branch as a source of mercury to its biota, caged fish may be introduced and monitored for it in conjunction with the biological indicator studies (Sect. 5). Alternatively, resident species unlikely to be immigrants from Poplar Creek, such as creek chubs or blacknose dace, niaj' be analyzed for mercury if populations are large enough that such coliections would not substantially affect them. 


\title{
5. BIOLOGICAL INDICATORS OF CONTAMINANT-RELATED STRESS
}

\author{
S. M. Adams and W. D. Crumby
}

\subsection{INTRODUCTION}

This report summarizes the results of the 1989 bioindicator study conducted in Mitchell Branch and three reference streams. Two approaches were used to evaluate the current health status of fish in Mitchell Branch: (1) analysis of functional biological response groups and (2) analysis of integrative bioindicator groups. The functional group approach involved analysis of five major groups of biotic responses to determine if differences in fish health existed between sites. The integrative bioindicator analysis involved the use of all bioindicators together within a multivariate context to investigate holistic responses of fish to stress and to aid in the identification of causative agents or mechanisms that may be affecting the health of fish populations in Mitchell Branch.

\subsection{METHODS}

\subsubsection{Sampling Procedures}

Sampling was conducted during summer of 1989 in Mitchell Branch (above weir) and three reference streams, including Brushy Fork located between Oliver Springs and Clinton (Anderson County, Tennessee), Hinds Creek between Clinton and Norris (Anderson County), and Paint Rock Creek (Loudon County, Tennessee). At each site, redbreast sunfish (Lepomis auritus) of all sizes and age groups were collected by electroshocking. Blood samples were taken from 12 to 15 of the larger male fish within 2 min after collection by puncturing the caudal vessels with a 20-gauge needle. Blood samples of $\sim 0.7 \mathrm{~mL}$ were obtained from all fish by using unheparinized $3-\mathrm{mL}$ vacutainers (Becton, Dickson, \& Co.). Each tube was labeled with a fish identification number and placed on ice for transport to the laboratory. All fish were processed for population-level analysis by recording their lengths and weights and removing a sample of scales for age and growth information. Fish not used for the blood and bioindicator analysis were returned to the stream alive.

\subsubsection{Analytical Procedures}

Total lengths and weights were recorded for fish transported from the field, and observations were also made on the general condition of the fish, such as presence or absence of disease, body and/or mouth sores, external parasites, and general appearance. Following sacrifice, the liver and spleen were removed from each fish for further analysis. A 100-mg section of liver was placed in a $20-\mathrm{mL}$ scintillation vial with 5-mL of Bouin's fixative for histopathological analysis. The remaining viscera (minus liver and spleen) were excised from the body cavity, and their total weight was recorded after all food material was removed from the stomach and intestine. The liver and visceral somatic-indices were calculated by dividing their respective weights by total body weight. The condition factor $(K)$ of each fish was calculated as $K=10^{5} W / L^{3}$, 
where $W$ is body weight $(\mathrm{g})$ and $L$ is total length $(\mathrm{cm})$.

\subsubsection{Serum analyses}

Blood collected in the unheparinized tubes was allowed to clot, transferred with Pasteur pipettes to $1.5-\mathrm{mL}$ conical microcentrifuge tubes labeled with the fish identification number, and centrifuged for $3 \mathrm{~min}$ in a 3eckman Microfuge. The clear supernatant (serum) was drawn off with clean pipettes and transferred to labeled 1-mL conical plastic tubes.

Indicators of organ dysfunction [creatinine, alanine aminotransferase (ALT) albumin, and urea nitrogen (BUN)] were analyzed following the methods described by Henry et al. (1974), Bergmeyer et al. (1978), Doumas (1972), and Tiffany et al. (1972) respectively. Cholesterol was analyzed by the method of Allain et al. (1974), and triglycerides were analyzed by the procedure of Bucolo and David (1973). Total serum proteins were measured by the Biuret method (NCCLS 1979); the procedures for this assay are described in the Roche Diagnostic Systems (1986) information package. All of these methods are enzymatic assays; the reagents for each assay were obtained from Roche Diagnostic Systems. All serum assays were performed with an automated Centrifugal Fast Analyzer System (Cobas-Fara, Hoffman La Roche Instruments, Inc.). Calibrations were made by using the Roche serum calibrator as the standard and Moni-Trol-ES Level 1 and 2 (American Dade, Miami, FL) as internal standards.

\subsubsection{Histopathological analyses}

The following histrpathological analyses were performed by the School of Veterinary Medicine, University of
California-Davis: (1) percentage of tissue occupied by parasites, (2) percentage of liver composed of necrotic parenchyma, (3) percentage of tissue composed of macrophage aggregates, and (4) percentage of liver occupied by functional parenchyma. These analyses were performed by using methods described by Hinton and Couch (1984).

\subsubsection{Detoxification enzymes}

Microsome isolation. Fish hepatic microsomes were prepared by differential centrifugation (McKee et al. 1983) with several modifications. Fish were sacrificed by severing the spinal cord, and the livers were immediately removed and blotted dry. Each liver sample was placed in icecold buffer $(0.25 \mathrm{mM}$ sucrose, $0.1 \mathrm{M}$ tris, $\mathrm{pH}$ 7.4). The tissues were ininced and then homogenized in 5 volumes of buffer with a motor-driven Potter-Elvehjem glass and Teflon homogenizer. The homogenatei were centrifuged in a J-21B Beckman centrifuge at $3000 \mathrm{~g}^{*}$ for $10 \mathrm{~min}$ and at $10,000 \mathrm{~g}$ for $20 \mathrm{~min}$. The resulting supernatants were then centrifuged at $105,000 \mathrm{~g}$ for $60 \mathrm{~min}$ in a Beckman L.3-50 ultracentrifuge. Microsomal pellets were resuspended in $0.1 \mathrm{M}$ tris buffer, $1 \mathrm{mM}$ EDTA, and $20 \%$ glycerol at $\mathrm{pH} 7.4$ by sonication for 10 to $15 \mathrm{~s}$ with a Braun Sonic 1510 at $50 \mathrm{~W}$. All operations were performed at 0 to $4^{\circ} \mathrm{C}$. The microsomes were frozen with liquid nitrogen and stored at $-120^{\circ} \mathrm{C}$; no significant change in 7-ethoxyresorufin O-deethylase (EROD) activity was detected after 6 months of storage at this temperature. The activity of fish microsomes stored under these conditions have been reported to be stable for 1 year (Forlin and Anderson 1985).

\footnotetext{
'Italic lower case $\boldsymbol{g}$ denotes standard acceleration due to gravity $\left(\sim 9.8 \mathrm{~m} \cdot \mathrm{s}^{-1} \cdot \mathrm{s}^{-1}\right)$.
} 
Enzyme assays. The activity of EROD was measured fluorimetrically at $30^{\circ} \mathrm{C}$ (Burke and Mayer 1974) and expressed as moles of resorufin per minute per milligram of microsomal protein. The final reaction buffer contained $80 \mathrm{mM}$ HEPES buffer ( $\mathrm{pH} 7.8$ ), $5 \mathrm{mM}$ magnesium acetate, $1.0 \mu M$ 7-ethoxyresorufin, $250 \mu M$ of reduced nicotinamide adenine dinucleotide phosphate (NADPH), and $100 \mu M$ EDTA. The concentration of total protein used in the enzyme assay ranged from 0.2 to $1.0 \mathrm{mg} / \mathrm{mL}$, depending on the activity of the sample. Proteins were measured by the Bio-Rad (Richmond, California) reagent method (Bradford 1976) with a Centrifugal Fast Analyzer System in which bovine serum albumin was used as a standard. Cytochrome P-450 and cytochrome $b_{s}$ content were each measured by their characteristic oxidized and reduced spectra. Cytochrome P-450 samples were oxidized with carbon monoxide and reduced with sodium dithionite (Johannesen and DePierre 1978). Cytochrome $b_{5}$ was reduced with NADH, and the $b_{5}$ assays were conducted prior to the P-450 analysis (Stegeman et al. 1979). NADPH-cytochrome $c$ reductase was assayed spectrophotometrically by the reduction of cytochrome $c$ using an extinction coefficient of $21.1 \mathrm{~cm} / \mathrm{m} M$.

\subsubsection{Population characteristics}

Scale samples were used to estimate the age and growth rates of redbreast sunfish in Mitchell Branch, Hinds Creek, and Brushy Fork. Approximately ten scales were removed from each fish by using a small pair of metal forceps. Scales were taken from the left side of the body near the tip of the pectoral fin and stored in small coin envelopes. Total length (centimeter), weight (gram), species, sample site, date, and tag number (if applicable) were recorded on each envelope.
Fish not used for blood samples and bioindicator analyses were returned to the stream alive after the scale sample was taken.

In the laboratory, scales from each fish were arranged in rows between two cellulose acetate slides. The slides were pressed together by using a hand-cranked laboratory press (Wildlife Supply Co.), which left an impression of each scale on one of the slides. Scales of the smallest fish (4-6 cm total length) were not pressed because they did not make an adequate impression on the cellulose acetate slides. Instead, they were arranged between two slides, which were taped together and read directly.

Scale impressions were analyzed by a single individual who used microfiche machine with a magnification of $43 \times$. Scales were measured and annulus marks identified according to the techniques outlined by Jerald (1983). The scale radius and the distance from the focus to each annulus were measured to the nearest $1 \mathrm{~mm}$. All scale samples from Brushy Fork and Mitchell Branch were analyzed, but because the Hinds Creek sample was so large, no more than $1 j$ fish were read for any $1-\mathrm{cm}$ size group. If there were 15 or less fish in a size group, all fish in that group were analyzed. Quality assurance of the scale samples was maintained by having $20 \%$ of the scale samples from each site analyzed by a second individual.

Fish were divided into $i-\mathrm{cm}$ size groups based on total length (TL), and length-frequency histograms were developed for each stream. Data from the scale analyses were used to assign fish to age groups. Mcan TL (in centimeters) and mean weight (in grams) at time of capture were calculated for each age group.

A length-weight relationship of redbreast sunfish in each stream was developed according to the formula: $\log W=\log _{10} a+\dot{i} \log _{10} L$; where $W$ is 

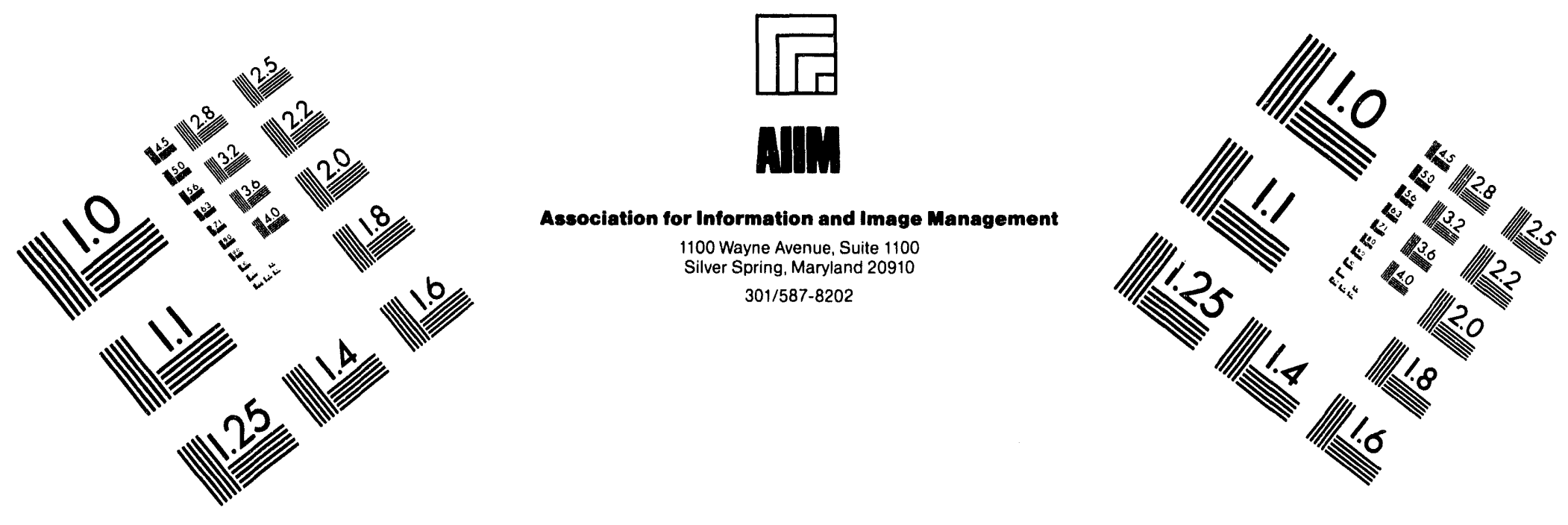

\section{Centimeter}

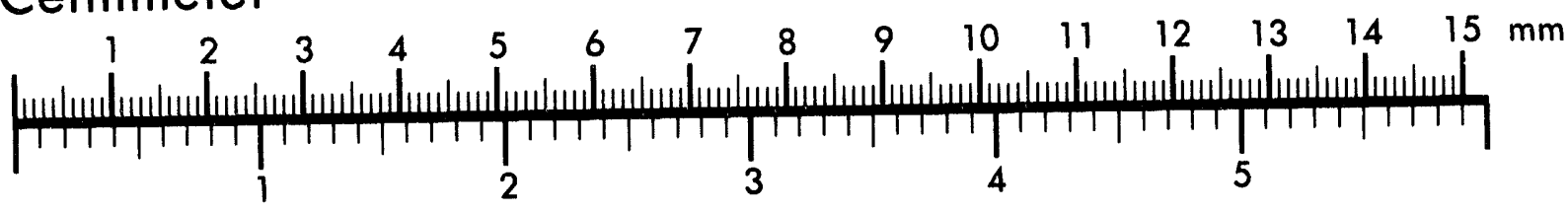
Inches
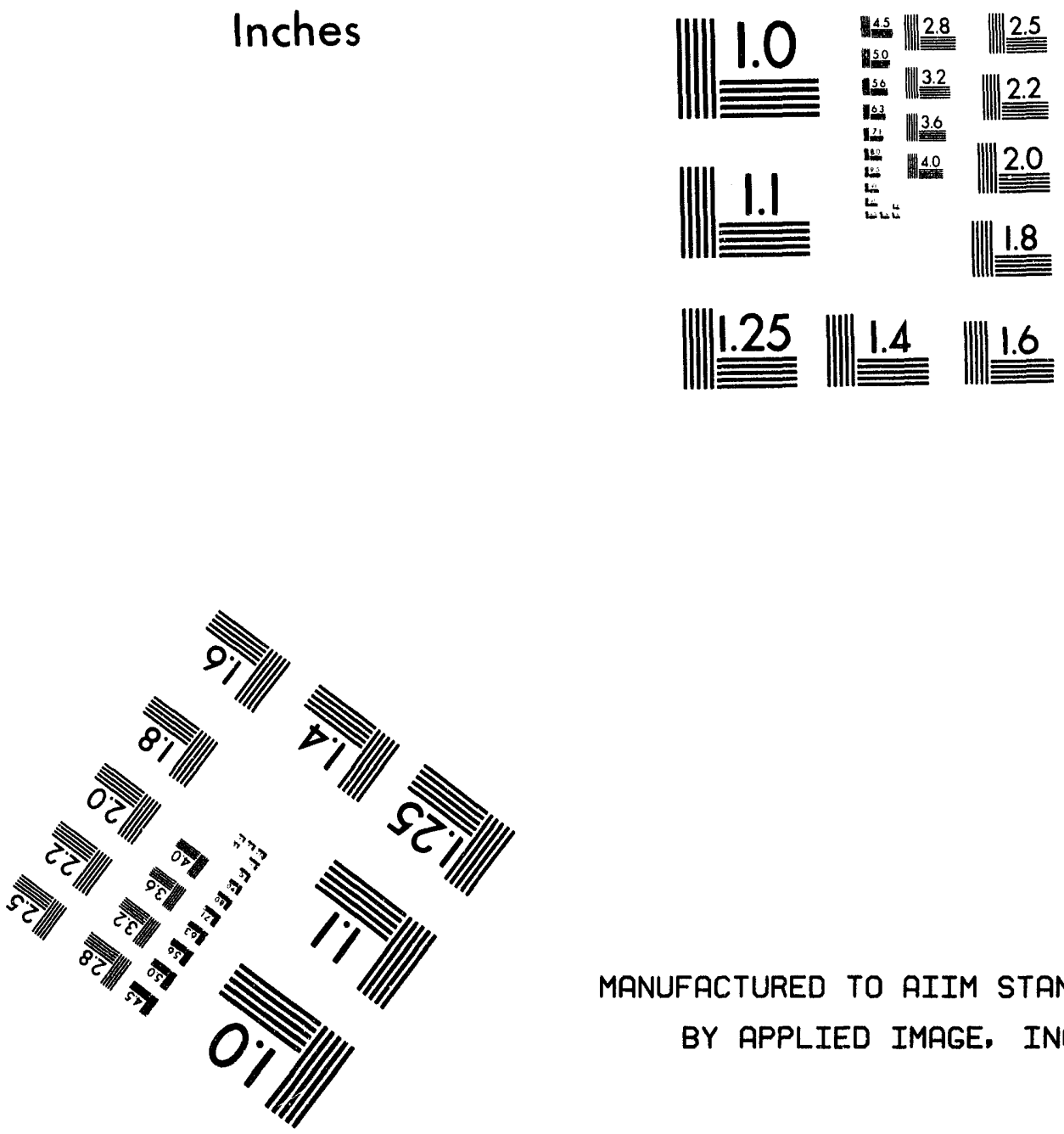

MANUFACTURED TO AIIM STANDARDS

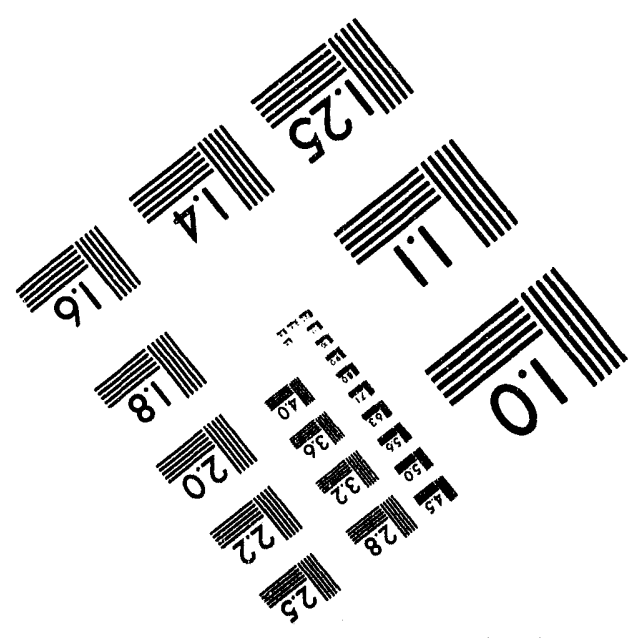



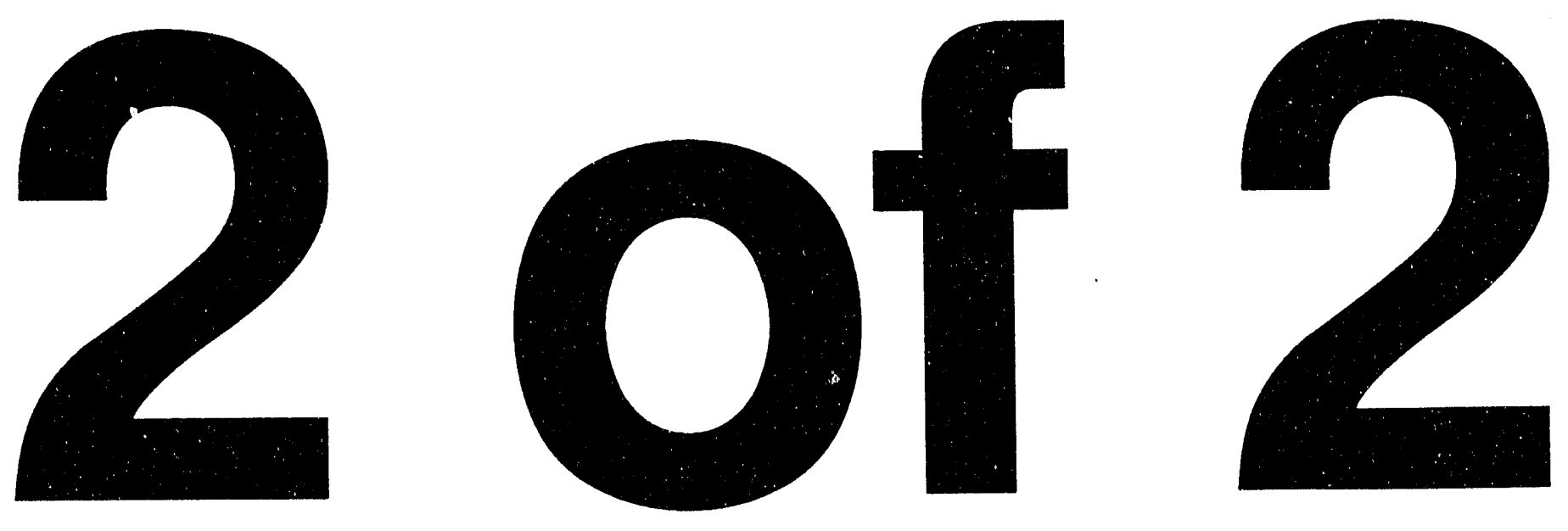


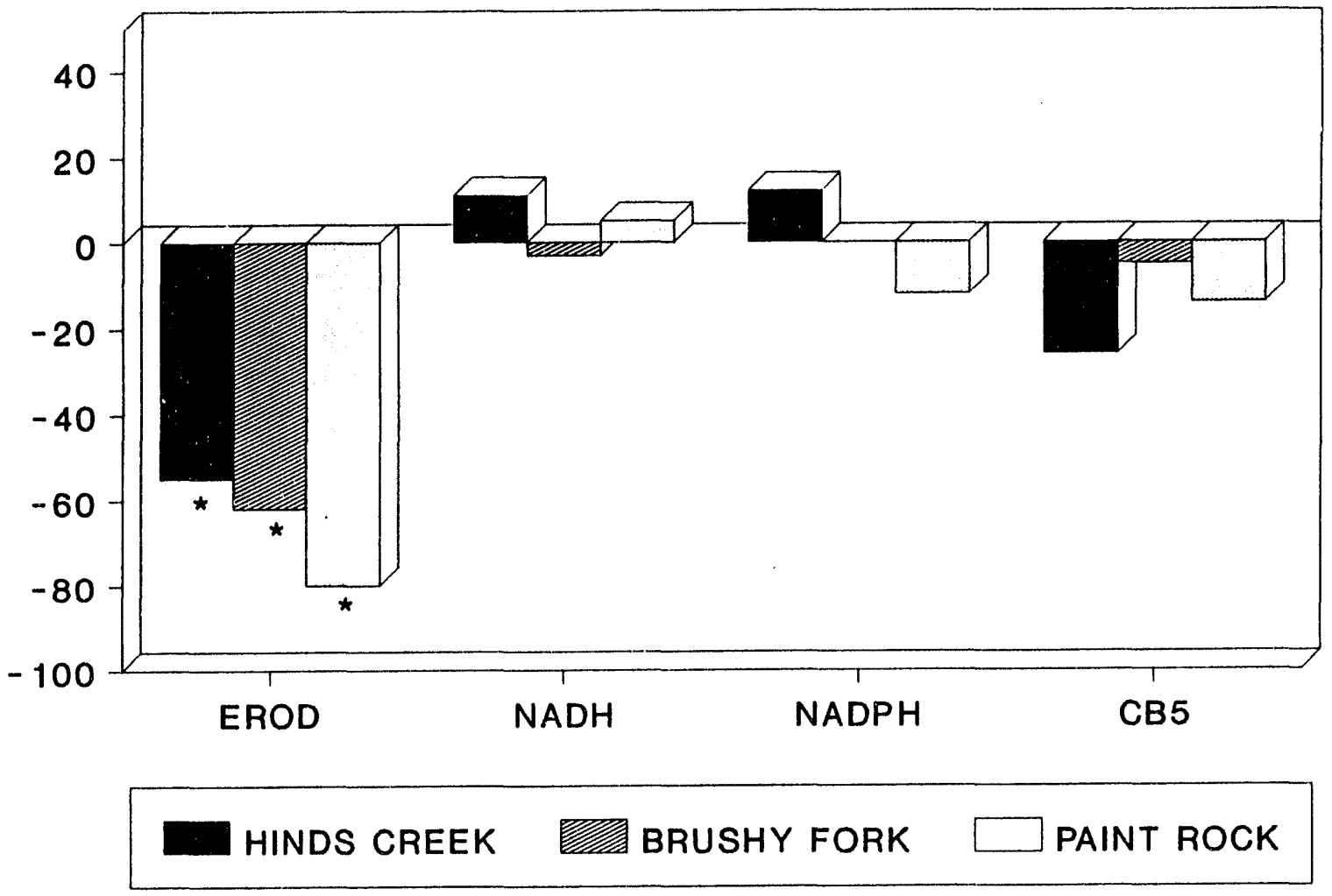

Fig. 5.2. Relative differences in the response of detoxification enzymes for redbreast sunfish from each of the reference sites compared with Mitchell Branch (the zero line!. Values above or below the zero line indicate that the response for fish from each of the reference streams was higher or lower, respectively, than that for fish from Mitchell Branch. Asterisks indicate values that were significantly different $(p<0.05)$ from Mitchell Branch.

damage, with elevated levels reflecting cirrhosis or obstructive jaundice. ALT activity in fish at all reference sites was slightly lower than that of fish from Mitchell Branch. However, these differences were not significant (Fig. 5.3).

BUN is the major end product of protein nitrogen metabolism in mammals and is generally used in the medical profession to diagnose renal disorders. Fish, however, produce a more diverse array of compounds as nitrogenous waste including, but not limited to, ammonia and urea. In addition to being excreted by the kidneys, some ammonia and urea is also excreted through the gills. Therefore, in diagnosing the effects of stress on fish, abnormal levels of urea in the blood may serve as an important indicator of gill dysfunction. In fish from Hinds Creek and Brushy Fork, BUN levels were significantly lower than in fish from Mitchell Branch. BUN levels in fish from Paint Rock Creek were lower than in fish from Mitchell Branch, but the difference was not statistically significant (Fig. 5.3). Because creatinine, an indicator of kidney dysfunction, was not elevated in Mitchell Branch fish, it is possible that water quality conditions in Mitchell Branch may be affecting gill function and not kidney function in these fish. 
ORNL-DWG 92-12923

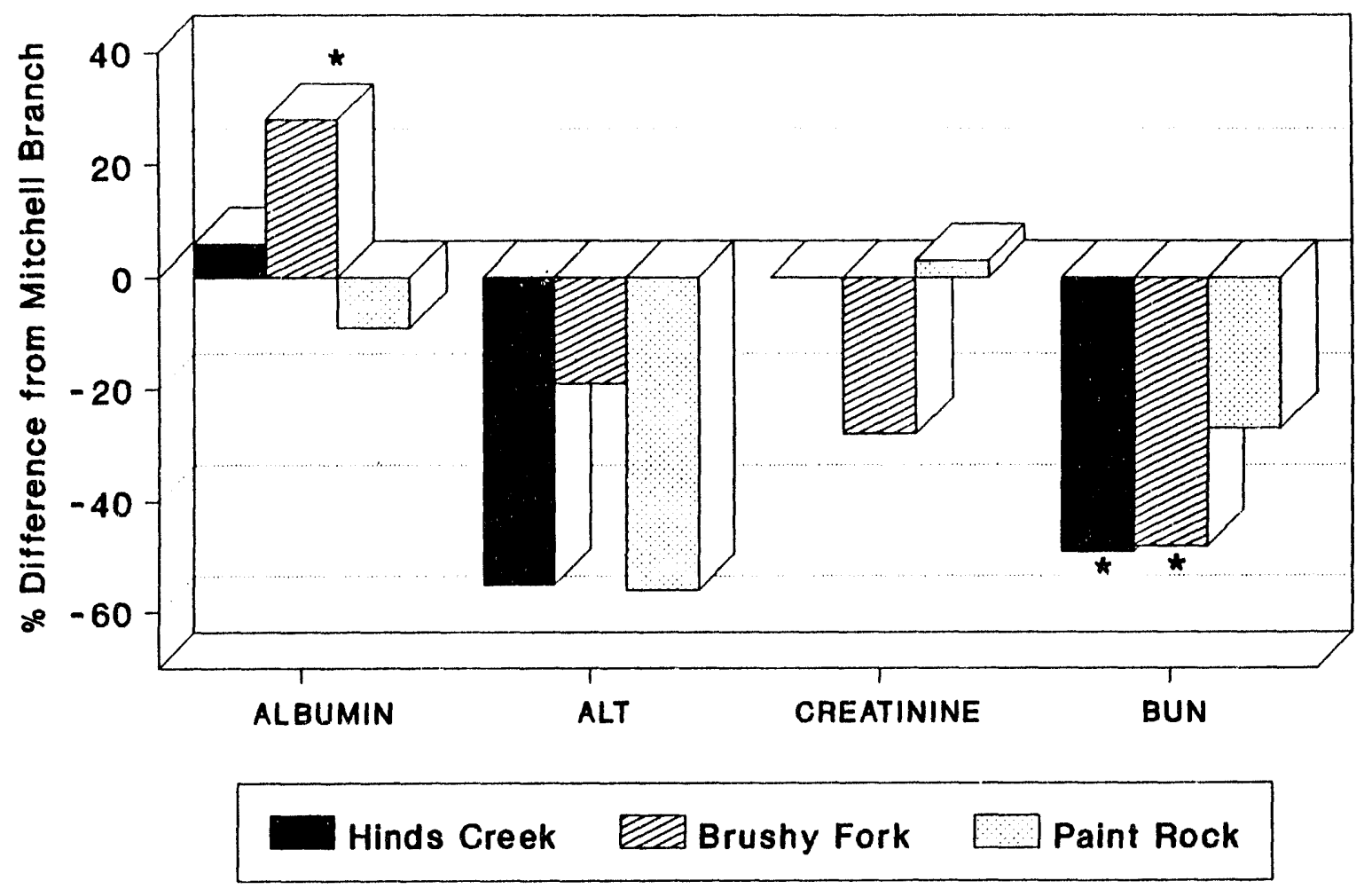

Fig. 5.3. Relative differences in the response of the organ dysfunction indicators for redbreast sunfish from each of the reference sites compared with Mitchell Branch (the zero line). Values above or below the zero line indicate that the response for fish from each of the reference streams was higher or lower, respectively, than that for fish from Mitchell Branch. Asterisks indicate values that were significantly different $(0<0.05)$ from Mitchell Branch. ALT = alanine aminotransferase, $B U N=$ blood urea nitrogen.

\subsubsection{Histopathological condition}

Indicators of histopathological condition in Mitchell Branch fish showed differences compared with reference fish, but these differences were not significant (Fig. 5.4). The percentage of the liver composed of encysted parasites (LPARS) was lower in Hinds Creek and Brushy Fork than in Mitchell Branch but was slightly elevated in Paint Rock Creek. The percentage of liver occupied by melanophage aggregates (LMACA), which are indicators of diseased or infected areas in the tissue, was lower in Hinds Creek and Brushy Fork than Mitchell Branch but also was slightly elevated in Paint Rock Creek redbreast. The percentage of liver actually occupied by functional parenchyma (LFPMA) was similar at all sites. There does seem, however, to be some indication of liver impairment in Mitchell Branch fish as indicated by elevated parasite loads and melanophage aggregates.

\subsubsection{Condition indices}

Three condition indices were measured as indicators of the general health of fish. The visceral-somatic index (VSI) provides information on energy 


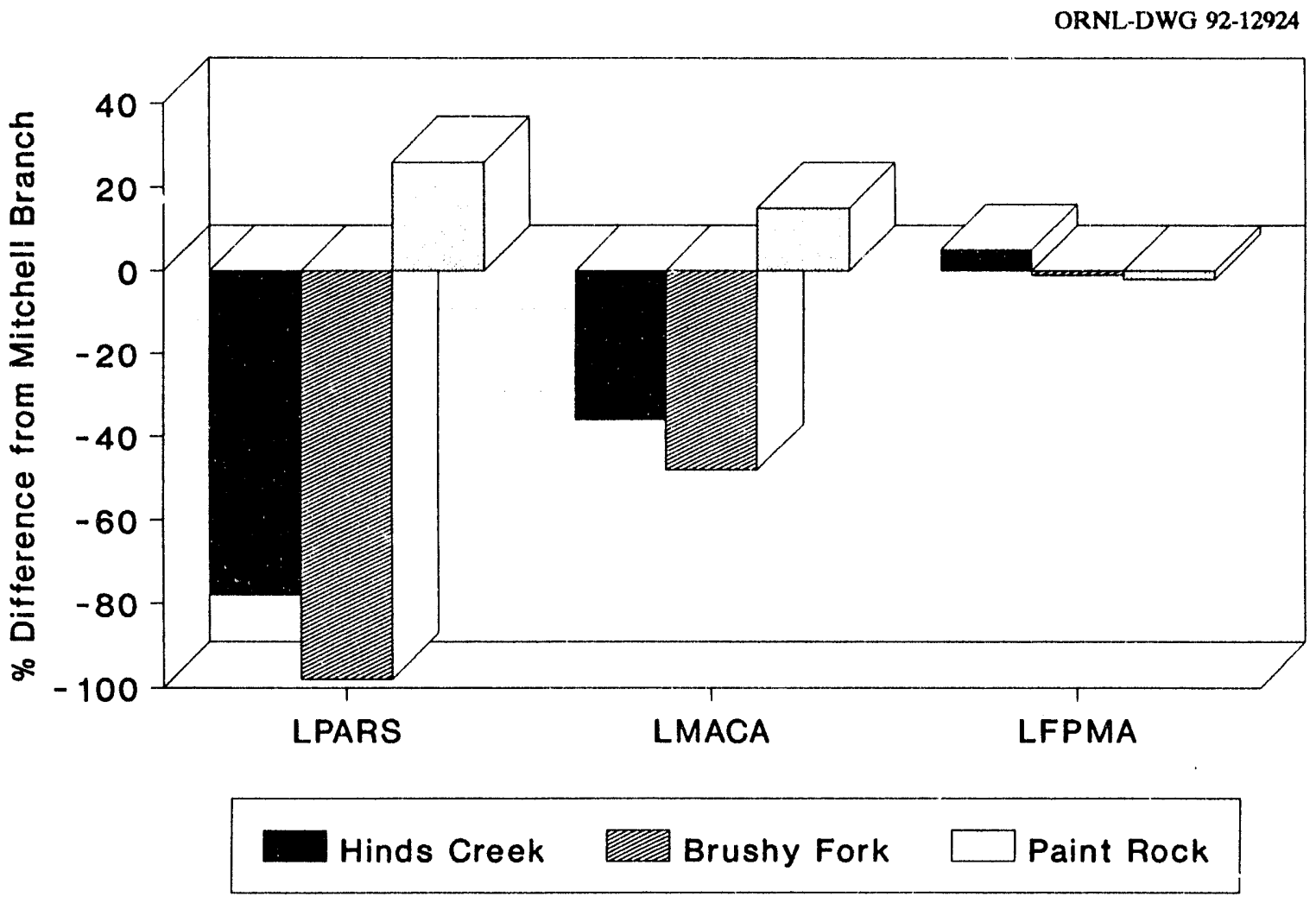

Fig. 5.4. Relative differences in histopathological condition for redbreast sunfish from each of the reference sites compared with Mitchell Branch (the zero line). Values above or below the zero line indicate that the response for fish from each of the reference streams was higher or lower, respectively, than that for fish from Mitchell Branch. Asterisks indicate values that were significantly different $(p<0.05)$ from Mitchell Branch. LPARS = liver parasites, $L M A C A=$ melanophage aggregates, LFPMA = functional parenchyma in liver.

stored as lipids in the mesenteries of the viscera. These lipids are important for long-term energy use and gonad maturation (Adams and McLean 1985). Additionally, the VSI generally reflects the level of total body lipids, which is used to indicate overall fat storage and general nutritional status of the fish. The VSI in redbreast from all three reference sites was significantly higher than in fish from Mitchell Branch (Fig. 5.5). Lower fat levels in Mitchell Branch fish may indicate that metabolic stress caused by contaminant exposure is greater in these fish and/or that less food is available.
The liver-somatic index (LSI) reflects both short-term nutritional status and metabolic energy demands (Heidinger and Crawford 1977, Adams and McLean 1985). The LSI is also sensitive to toxicant stress, and liver enlargement resulting from hyperplasia (increase in cell number) and hypertrophy (increase in cell size) has been reported in fish exposed to toxic compounds (Fletcher et al. 1982, Heath 1987, Addison 1984). The latter may be applicable to redbreast in Mitchell Branch because the LSI was higher at this site than for redbreast from all three reference streams, although the difference was not statistically significant (Fig. 5.5). 
ORNL-DWG 92-12925

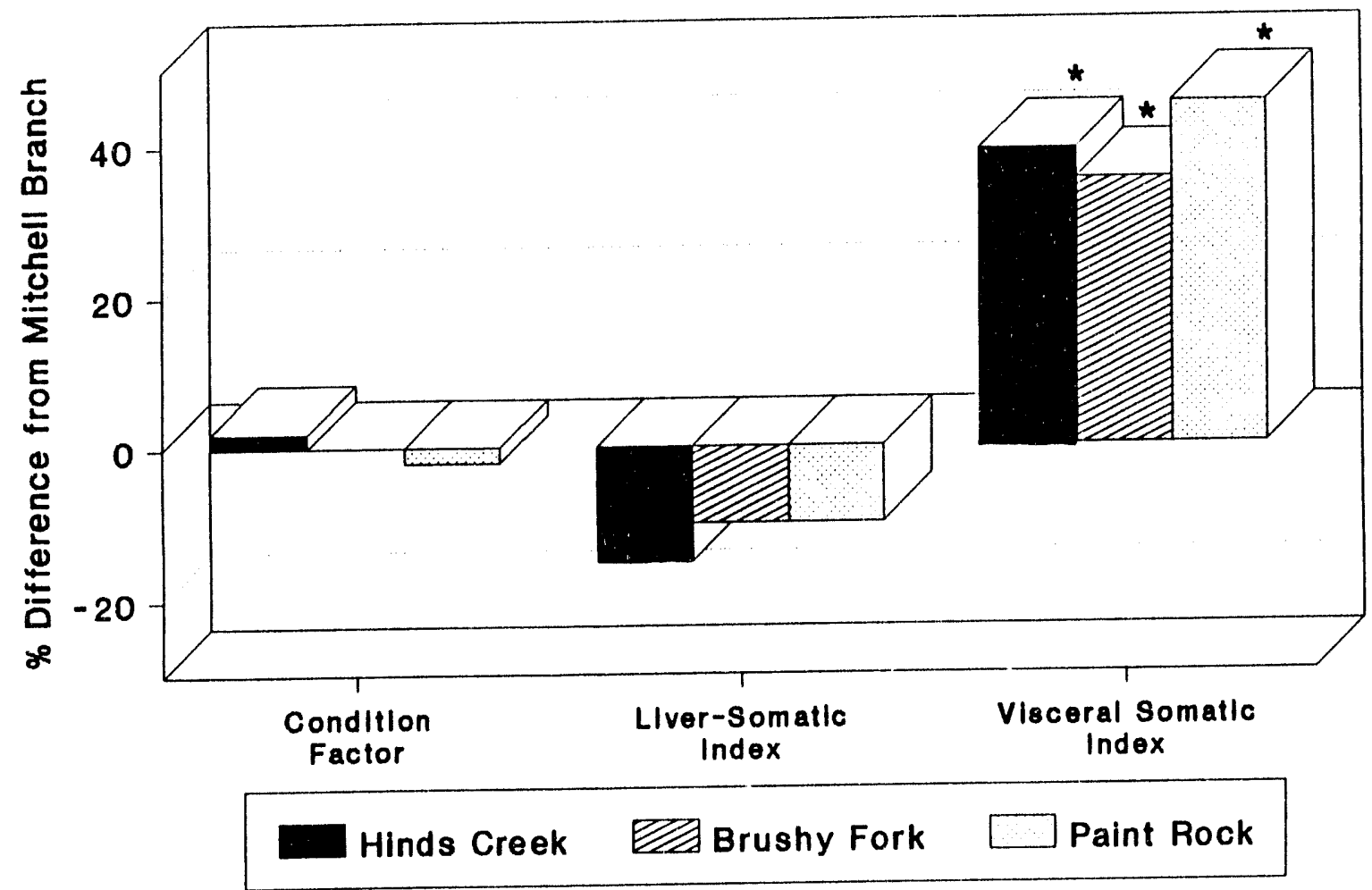

Fig. 5.5. Relative differences in the condition indices for redbreast sunfish from each of the reference sites compared with Mitchell Branch (the zero line). Values above or below the zero line indicate that the response foi fish from each of the reference streams was higher or lower, respectively, than that for fish from Mitchell Branch. Asterisks indicate values that were significantly different $(p<0.05)$ from Mitchell Branch.

The condition factor (CDFC) is a generalized indicator of the overall health or "plumpness" of a fish and can reflect the integrated effect of both nutritional status and metabolic impairment caused by stress. The condition factor is relatively insensitive to changes in lipid stores (Adams and McLean 1985). This feature of the index may explain why the CDFC values were similar for fish from Mitchell Branch and the three reference sites.

\subsubsection{Nutritional indices}

An evaluation of the nutritional status of the organism is important for interpret- ing the nature of the effects of contaminant stress on fish. Three indicators were used as measures of nutritional condition, including the percentage of the stomach and intestine containing food and the level of serum triglycerides. Fish from Hinds Creek and Paint Rock Creek had at least 45\% more food in their digestive systems than fish from Mitchell Branch, whereas fish from Brushy Fork had slightly less food (Fig. 5.6). None of these differences, however, were statistically significant.

Serum triglycerides were much higher in Brushy Fork and Paint Rock Creek fish than they were in fish from Mitchell Branch, indicating either higher feeding levels (food quantity) or better nutritional 


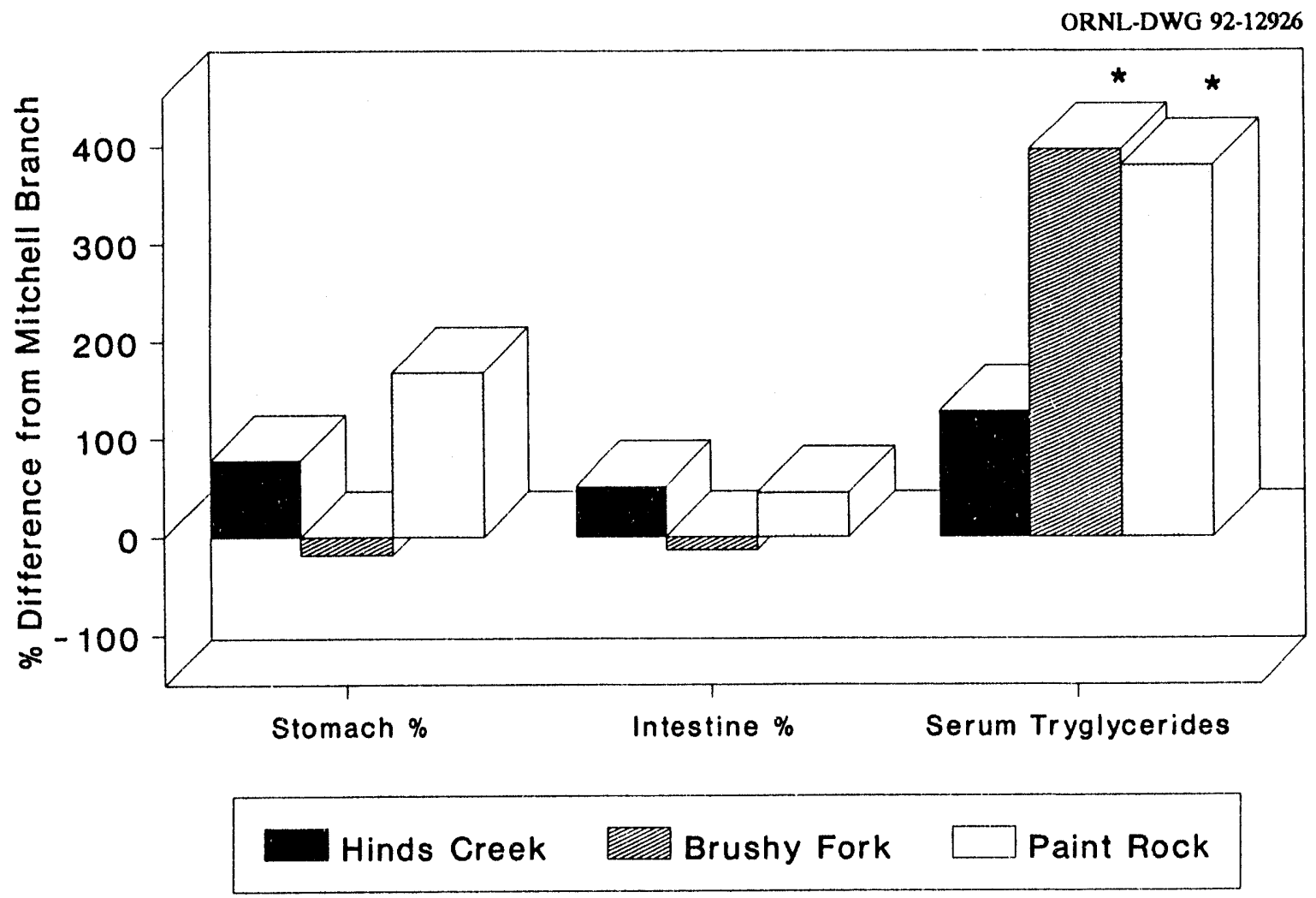

Fig. 5.6. Relative differences in the response of the nutritional indices for redbreast sunfish from each of the reference sites compared with Mitchell Branch (the zero line). Values above or below the zero line indicate that the response for fish from each of the reference streams was higher or lower, respectively, than the same response for fish from Mitchell Branch. Asterisks indicate those values that were significantly different $(p<0.05)$ from Mitchell Branch.

content (food quality) of available food for fish from these reference sites (Fig. 5.6). The higher levels of triglycerides in fish from the reference sites suggest that these fish were in better nutritional condition than fish from Mitchell Branch.

\subsubsection{Integrated Bioindicator Analysis}

Canonical discriminant analysis can be an informative approach for $r$ termining the overall response of fish to stress. This method includes all the bioindicators within a multivariate context and provides a graphical representation of the positions and orientations of the sites relative to each other. This integrated approach to evaluating the effects of water quality on fish populations has the primary advantage of providing an integrative assessment of the health of fish based on multiple rather than individual indicators.

The most obvious feature of the site responses shown in Fig. 5.7 is that the integrated response of fish from the three reference streams is distinct from that of redbreast from Mitchell Branch. The integrated responses of fish from Brushy Fork and Hinds Creek are not significantly different, as shown by overlapping of their 95\% confidence radii $(p<0.05)$. As indicated by the differences in the linear distances between the site means 
ORNL-DWG 92-12927

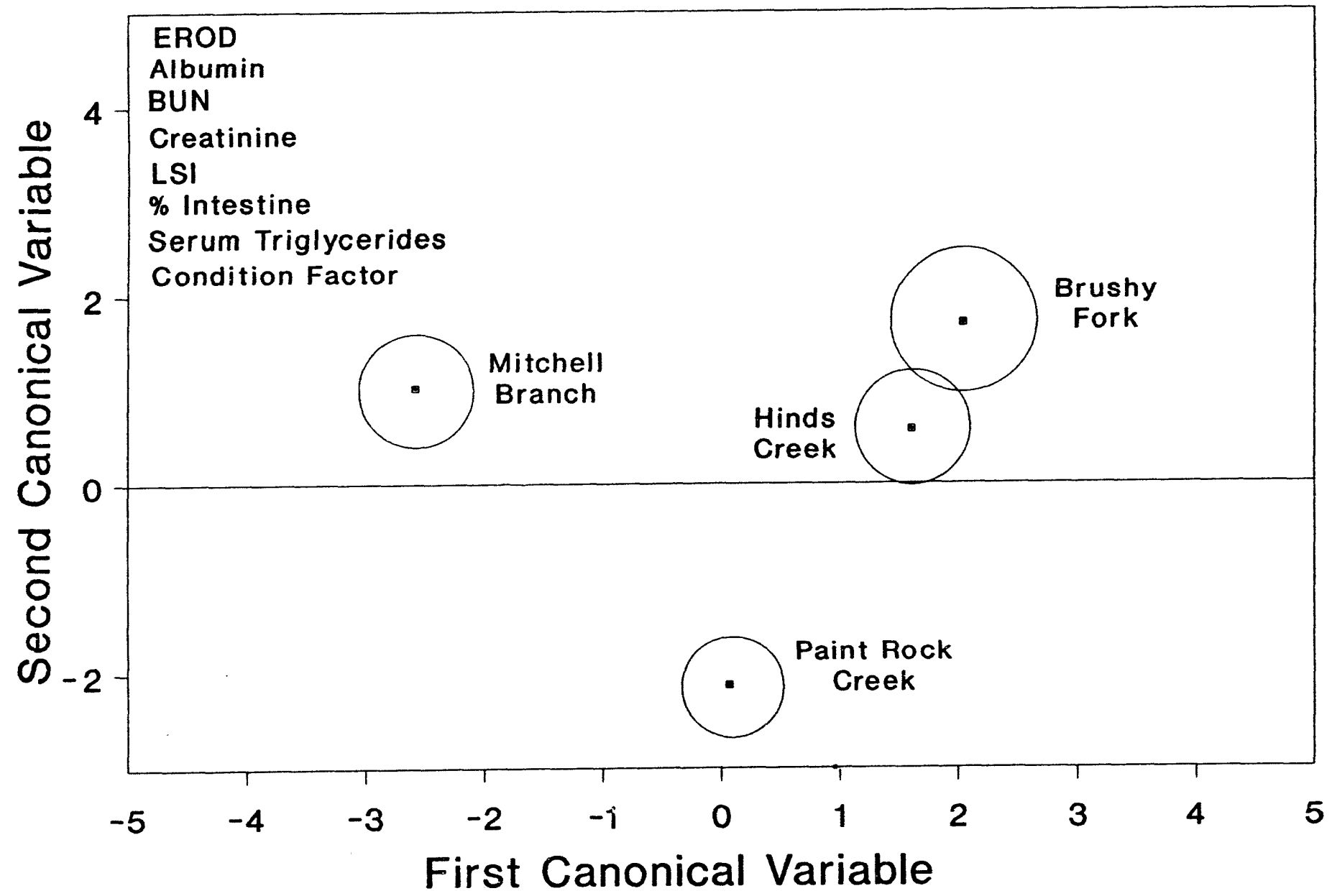

Fig. 5.7. Segregation of integrated health responses for redbreast sunfish from three reference sites and Mitchell Branch. Circles represent site means and the $95 \%$ confidence radii of the site means. Also shown are the variables used for discriminating among these sites. 
(midpoints of circles), redbreast from Paint Rock Creek were the most similar to redbreast from Mitchell Branch, whereas fish from Brushy Fork were least similar to fish from Mitchell Branch.

Multivariate selection. A multivariate selection procedure was used to identify the individual variables that best discriminated between the health status of fish at the various sites. The variables examined that were the most important in discriminating among sites are indicated on Fig. 5.7. These eight variables consist of representative indicators from four of the five functional groups (see Sect. 5.3), including one detoxification enzyme, three organ dysfunction indicators, two condition indices, and two nutritional indices. This analysis illustrates the importance of including bioindicators at several levels of biological organization when evaluating the integrated responses of fish to environmental stress.

\subsubsection{Population-Level Analysis}

Length-frequency histograms indicated that the population structure of redbreast sunfish at Mitchell Branch is different from that at the reference sites (Fig. 5.8). Only 25 fish could be collected from Mitchell Branch, all of which were greater than $11 \mathrm{~cm}$ in total length. This atypical distribution of sizes in the Mitchell Branch population may indicate a problem with reproduction or recruitment. The habitat in Mitchell Branch appears inadequate for successful spawning. Like most species of sunfish, redbreasi prefer sand and gravel over other substrate types, such as silt or detrital material, for nest construction (Davis 1972). However, much of the stream bottom at Mitchell Branch is covered with silt, with accumulations several centimeters deep. Even if hatching were successful, predation by larger fish in the system could limit survival of young.
An alternative explanation for lack of smaller fish at Mitchell Branch might have to do with the immigration of fish from Poplar Creek. The larger fish may swim past or over the weir in times of high water flow. For example, water levels were observed to be 0.5 to $1 \mathrm{~m}$ over the top of the K-1700 weir on two occasions during heavy rains in June 1989. The only apparent contradiction to this immigration hypothesis is that in 1989 , electrofishing samples below the weir and downstream in Poplar Creek yielded very few redbreast sunfish, none over $10 \mathrm{~cm}$. The small number of redbreasts at Mitchell Branch may be directly related to the size of the sample area. From the weir to the upstream boundary of the sample site is a distance of $\sim 100 \mathrm{~m}$, which may limit the number and bioma $s$ of fish this stream section can support in terms of food and habitat resources.

Although 227 fish were collected at Hinds Creek, only 159 were randomly selected for scale analysis. All scale samples from Brushy Fork (91) and Mitchell Branch (25) were analyzed. Fish from 1 to 7 years old were observed from these three streams, but most were from age classes 1 through 3 (Table 5.1). In age classes 4-7, sample sizes were quite small, and statistical comparisons were not possible. This was also true of age 1 fish in Mitchell Branch. Thus, statistical comparisons were made only for growth rates of fish in age classes 2 and 3, although data from other age groups are displayed in Figs. 5.9 through 5.11.

Sunfish from Mitchell Branch were generally larger at each age than those at the reference sites (Figs. 5.9 and 5.10). Because stream area is limited and substrate is not ideal for benthic food production, habitat does not seem to be conducive to favorable growth rates. Lack of competition for resources could be a reason for the larger size and weight of fish at Mitchell Branch. Electrofishing 
ORNL-DWG 92-12928
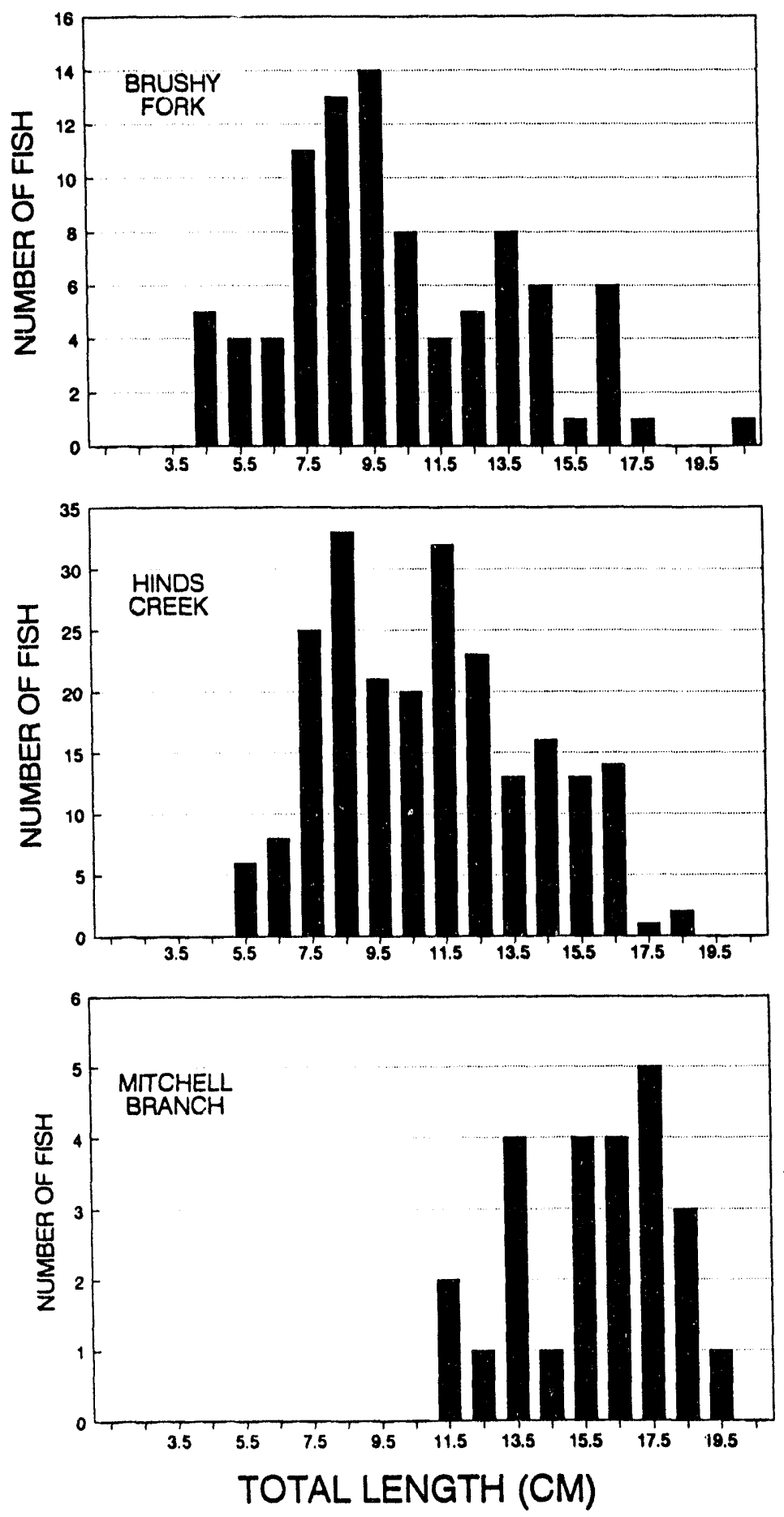

Fig. 5.8. Length frequency of redbreast sunfish collected in Mitchell Branch, Hinds Creek, and Brushy Fork during August 1989. Bars are plotted at the midpoints of $1-\mathrm{cm}$ size groups. 
Table 5.1. Number of redbreast sunfish scale samples eramined for each age group at each sample site

\begin{tabular}{|c|c|c|}
\hline Sample site & Age & Sample size \\
\hline Mitchell Branch & $\begin{array}{l}1 \\
2 \\
3 \\
4 \\
5\end{array}$ & $\begin{aligned} n & =2 \\
n & =13 \\
n & =8 \\
n & =1 \\
n & =1 \\
\text { Total } & =25\end{aligned}$ \\
\hline Brushy Fork & $\begin{array}{l}1 \\
2 \\
3 \\
4 \\
5 \\
6\end{array}$ & $\begin{aligned} n & =32 \\
n & =23 \\
n & =28 \\
n & =3 \\
n & =3 \\
n & =2 \\
\text { Total } & =91\end{aligned}$ \\
\hline Hinds Creek & $\begin{array}{l}1 \\
2 \\
3 \\
4 \\
5 \\
6 \\
7\end{array}$ & $\begin{aligned} n & =38 \\
n & =53 \\
n & =58 \\
n & =6 \\
n & =0 \\
n & =3 \\
n & =1 \\
\text { Total } & =159\end{aligned}$ \\
\hline
\end{tabular}

samples revealed that no competing species of fish were present in substantial numbers. Only a few small bluegill and green sunfish were found; therefore, redbreast basically compete with each other for available food. Redbreast sunfish at both reference sites must also compete with bluegill, rockbass, suckers, and several species of Notropis. Mitchell Branch redbreast could also have attained their size in the larger. Poplar Creek system before immigrating into Mitchell Branch.

The mean instantaneous growth rate $(G)$ of age 1 redbreast sunfish in 1988 was significantly higher $(p<0.05)$ at Mitchell
Branch than at the reference sites (Fig. 5.11). However, no significant difference in the growth rate was found among the reference sites. Age 2 fish in Mitchell Branch grew at a significantly lower rate in 1988 than did fish at either of the reference sites. As for age 1 fish, there were no significant differences in growth rates of age 2 fish among the reference sites. This pattern suggests that in 1988 there could have been unequal availability of food resources for different size fish in Mitchell Branch. The smaller age 1 fish grew well, but the larger fish were apparently less able to benefit from 


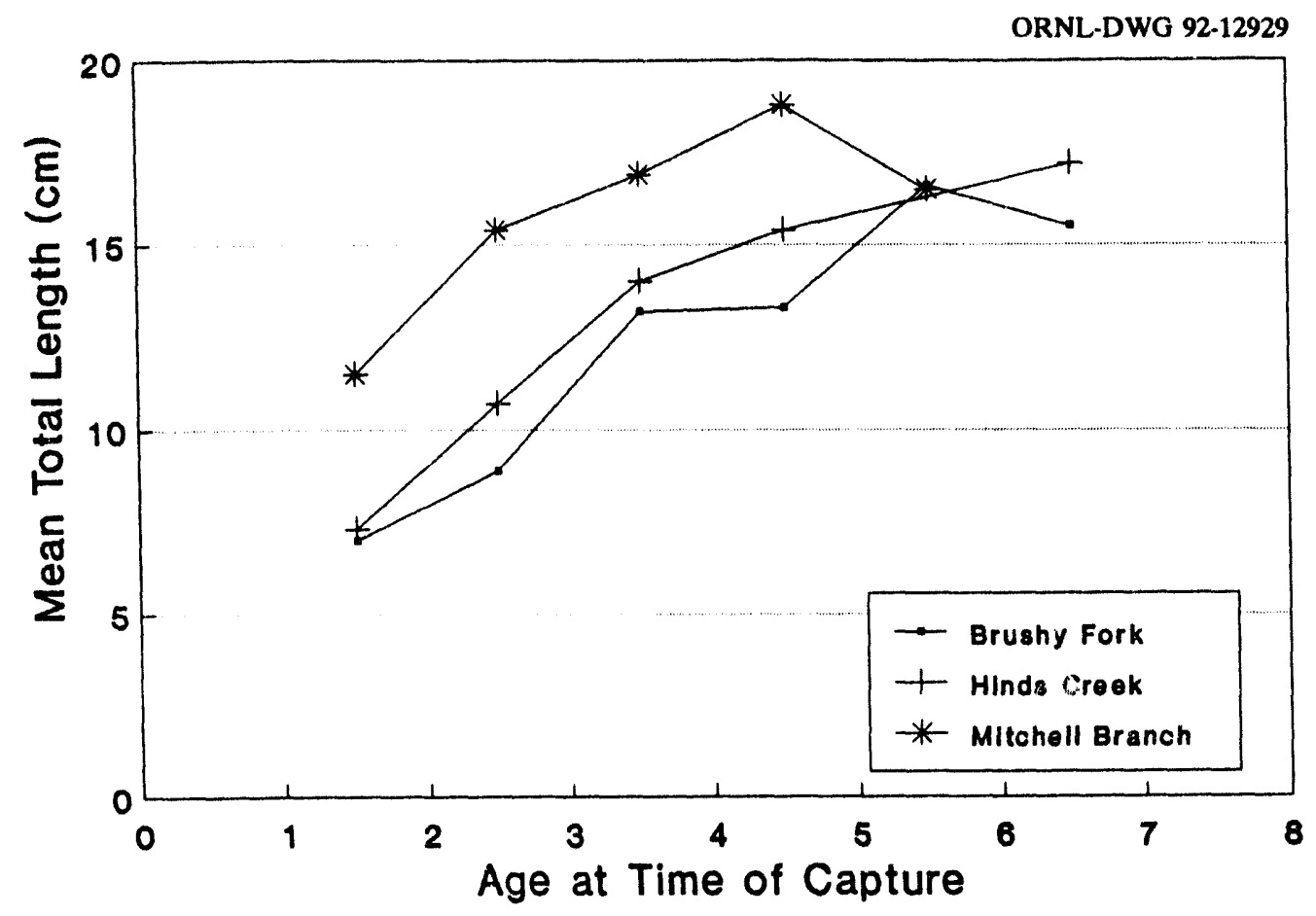

Fig. 5.9. Mean total length of redbreast sunfish in each age group in August 1989 at the three sample sites.

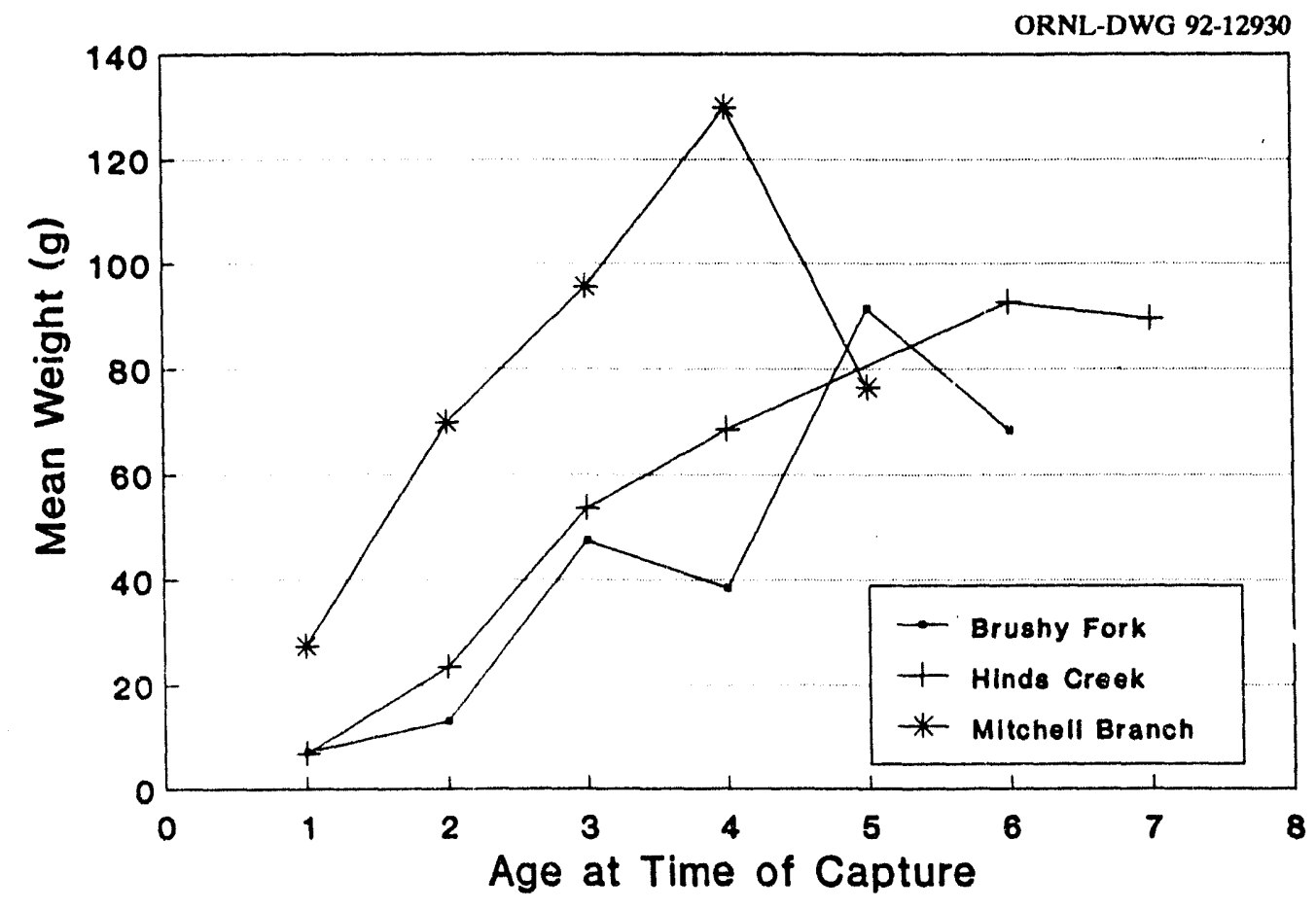

Fig. 5.10. Mean weight of redbreast sunfish in each age group August 1989 at the three sample sites. 
ORNL-DWG 92-12931

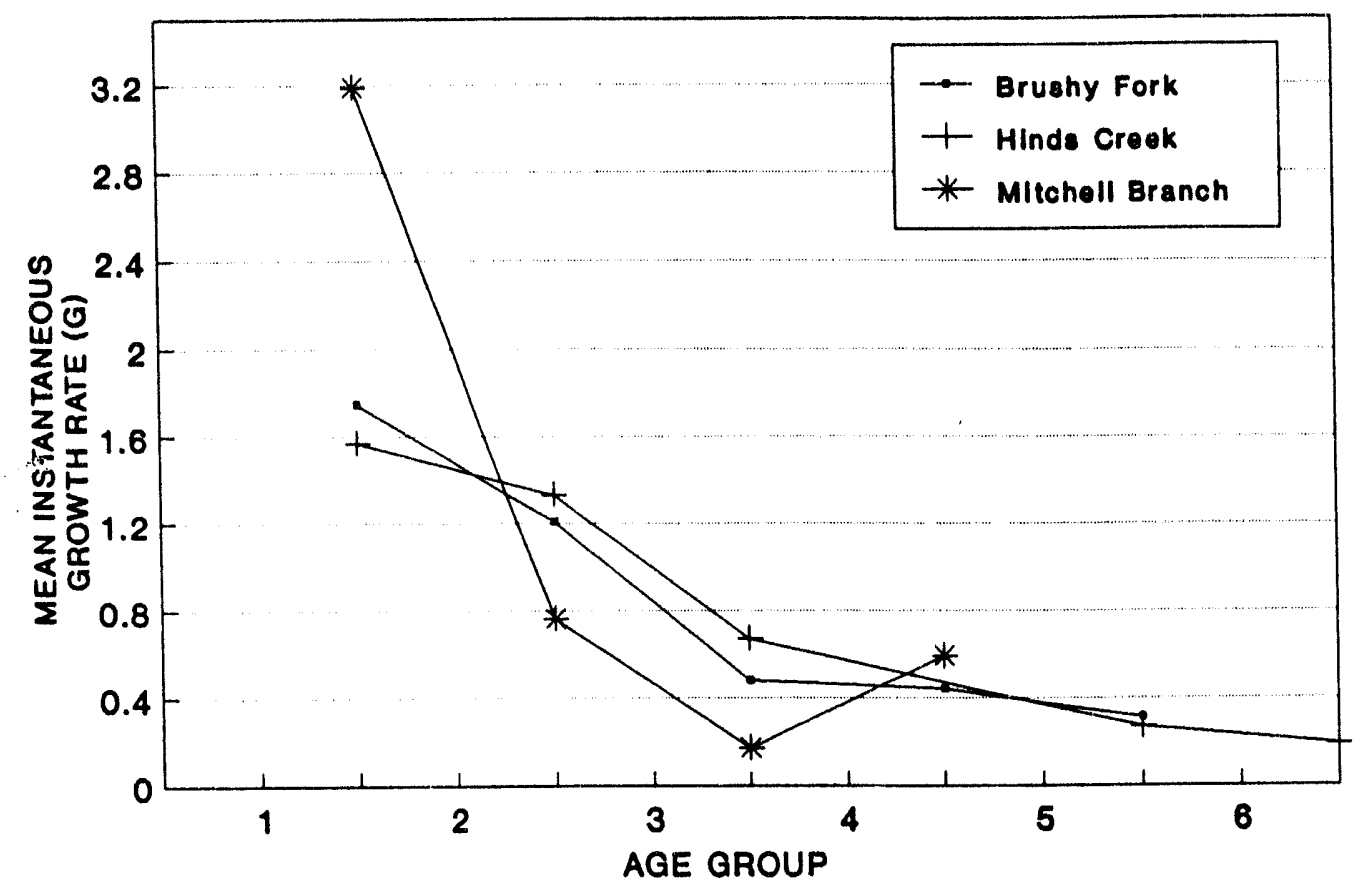

Fig. 5.11. Mean instantaneous growth rate (G) of redbreast sunfish in 1988 at the three sample sites.

the same resources. Because of the need for larger prey iteins and the limited area of habitat, competition may have been greater between larger fish.

\subsubsection{Summary and Synthesis}

Several conclusions can be drawn from the comprehensive analysis of the 1989 bioindicator data from Mitchell Branch. Redbreast sunfish in Mitchell Branch appear to be physiologically stressed. On a short-term basis, the effects of contaminant exposure are reflected in gill and possibly liver dysfunction for fish inhabiting Mitchell Branch. Longer-term stress is manifested by changes in histopathological condition and overall condition or health. The reproductive competence of the redbreast sunfish population in Mitchell Branch may have been compromised, as evidenced by the lack of small individuals in the population. Some of the stress responses may have been the result of the indirect effects of water quality manifested through the food chain.

The integrated response of fish from Mitchell Branch was distinctly different from that of fish from the three reference streams. Although the integrated response was not the same for all three reference sites, they were all dissimilar to Mitchell Branch by approximately the same amount, indicating impaired health of fish in this stream.

When integrated, bioindicators representative of four functional groups permitted Mitchell Branch fish to be statistically separated from fish at the reference sites. This analysis demonstrates the importance of incorporating bioindicators from several levels of biological 
organization into biological monitoring programs. Such an integration is needed to effectively evaluate the effects of contaminant-related stress on fish populations.

\subsection{FUTURE STUDIES}

The annual bioindicator study of fish in Mitchell Branch will continue with one major change initiated during the 1990 sampling period. At that time, reproductive studies were initiated to assess the effects of contaminant stress on reproductive success. Reproductive competence will be evaluated based on measurements of gonad condition (e.g., quality and quantify of eggs), sizes and timing of clutches, hormone levels, and gonad histology.

In addition, a new system (fish health assessment index) of evaluating fish health is currently being developed to supplement the quantitative bioindicator studies. This system, which provides a general health profile of a fish population, can detect departures from normal in growth, bioenergetic state, general homeostasis, nutritional status, and presence of infectious agents. This evaluation will be used to help test the hypotheses that bioindicator responses of fish result directly from contaminant exposure and are not mediated indirectly through the food chain. 


\title{
6. INSTREAM ECOLOGICAL MONITORING
}

\author{
J. G. Smith and M. G. Ryon
}

The objectives of the instream ecological monitoring task (Task 4 of BMAP as described in Loar et al. 1991b) are to (1) characterize spatial and temporal patterns in the distribution and abundance of the benthic macroinvertebrate and fish populations and (2) document the effects of new pollution abatement facilities on community structure and function. This task consists of two components: (1) benthic macroinvertebrate studies (Subtask 4a) and fish population studies (Subtask 4b); results to date for these studies are presented in Sects. 6.1 and 6.2 respectively.

\subsection{BENTHIC MACROINVERTE- BRATES (J. G. Smith)}

\subsubsection{Introduction}

During the initial year of BMAP (August 1986 through July 1987) the benthic invertebrate community of the middle and lower reaches of Mitchell Branch exhibited characteristics indicative of moderately to severely degraded water quality (Smith et al. 1993). These affected reaches were generally characterized by low taxonomic diversity and richness and the absence or low relative abundance of some major taxonomic groups that are relatively intolerant of pollution (e.g., Ephemeroptera, Plecoptera, and Trichoptera). During the second year of BMAP (August 1987 through July 1988), benthic invertebrate studies continued as in the first year with the specific objectives of (1) further characterizing the benthic macroinvertebrate community in
Mitchell Branch and documenting the impacts on this community from past and current Oak Ridge K-25 Site operations and (2) continuing efforts to identify causal factors responsible for any observed adverse impacts. This report includes results of samples collected from Mitchell Branch from August 1987 through January 1988; thus, in the absence of a complete year of data, conclusions drawn from these data are considered tentative.

\subsubsection{Materials and Methods}

Benthic macroinvertebrates were sampled at approximately monthly intervals from August 1987 through July 1988 at six sites in Mitchell Branch (see Fig. 2.1); the upstream-most site (MIK 1.43) served as a reference site. Three random quantitative samples were collected from each site with a Surber bottom sampler $\left(0.09 \mathrm{~m}^{2}\right)$ fitted with a $363-\mu \mathrm{m}$-mesh collection net. Each replicate sample was placed in a prelabeled, glass jar and preserved in $80 \%$ ethanol; the ethanol was replaced with fresh ethanol within 1 week of collection. The laboratory procedures used to process these samples are described in Smith et al. (1993).

Supplemental information on water quality and stream characteristics were also recorded at the time of sampling. Temperature, conductivity, dissolved oxygen, $\mathrm{pH}$, and turbidity were measured with an Horiba Model U-7 Water Quality Checker. Water depth, location within the riffle area (distance from permanent headstakes on the stream bank), relative stream velocity (visually determined as very slow, 
slow, moderate or fast), and substrate type based on a modified Wentworth particle size scale (Loar et al. 1985) were recorded for each sample.

All calculations and statistical analyses were performed with the use of the Statistical Analysis System (SAS 1985a, 1985b). The Shannon-Wiener index $\left(H^{\prime}\right)$ was used to calculate taxonomic diversity of benthic macroinvertebrates at each site (Pielou 1977):

$$
H^{\prime}=-\Sigma P_{j}\left(\log _{2} P_{j}\right),
$$

where $P_{j}$ is the proportion of the benthic invertebrate community made up of species $j$. The $H^{\prime}$ values of 3 or greater are typical of clean-water communities, whereas values of 1 to 3 are usually associated with moderate pollution and values of less than 1 characterize heavily polluted water (Platts et al. 1983).

Statistical comparisons were performed on transformed data $\left[\log _{10}(X+1)\right]$ (Elliott 1977). Mean values for density; biomass; number of taxa (taxonomic richness); number of Ephemeroptera, Plecoptera, and Trichoptera taxa (EPT richness); and taxonomic diversity were compared separately with a two-way ANOVA with site and date as the main effects. Significant site differences $(p<0.05)$ were then separated with a Tukey studentized range test. Betweenyear comparisons within each site were made with a one-way ANOVA with year as the main effect.

\subsubsection{Results}

\subsubsection{Taxonomic composition}

A checklist of the benthic invertebrates collected from Mitchell Branch from August 1987 through January 1988 is given in Appendix E, Table E.1. Over 143 taxa, of which 124 were insects, were collected in quantitative samples from Mitchell Branch. Ten orders of insects were collected, including Collembolla (springtails), Ephemeroptera (mayflies), Odonata (dragonflies and damselflies), Plecoptera (stoneflies), Hemiptera (true bugs), Megaloptera (alderflies and fishflies), Trichoptera (caddisflies), Coleoptera (beetles), Hymenoptera (wasps), and Diptera (true flies). The order Diptera was the most taxonomically rich group, with 77 representative taxa, of which 55 were from the family Chironomidae. The remaining insect orders were represented by nine or fewer taxa. Several insect taxa were not collected from sites downstream of the reference site (MIK 1.43). Particularly notable at these downstream sites was the absence or rare occurrence of most or all representative taxa that are generally intolerant of pollution, such as the Ephemeroptera, Plecoptera, and Trichoptera.

The noninsect taxa collected included Turbellaria (flatworms or planarians), Nematoda (roundworms), Oligochaeta (aquatic earthworms), Copepoda (copepods), Ostracoda (seed shrimp), Isopoda (aquatic sow bugs), Amphipoda (sideswimmers), Decapoda (crayfish), Hydracarina (water mites), Gastropoda (snails), and Bivalvia (mussels and clams) (Table E.1). As for the insects, several noninsect taxa (e.g., Isopoda, Amphipoda, Bivalvia) collected at MIK 1.43 were not collected at those sites further downstream.

\subsubsection{Density and biomass}

Mean density and biomass of the benthic macroinvertebrates at each sampling site in Mitchell Branch during the first 6 months of the second year of BMAP are presented in Table 6.1. Both density and biomass were significantly higher at MIK 1.43 than at each downstream 
Table 6.1. Benthic macroinvertebrate density, biomass, taxonomic richness, EPT richness, and taxonomic diversity in Mitchell Branch, August 1986-January 1987 (Year 1) and August 1987-January 1988 (Year 2)

\begin{tabular}{|c|c|c|c|c|c|}
\hline Site & $\begin{array}{c}\text { Density } \\
\text { (number } / 0.1 \mathrm{~m}^{2} \text { ) }\end{array}$ & $\begin{array}{c}\text { Biomass } \\
\left(\mathrm{mg} / 0.1 \mathrm{~m}^{2} \text { wet } \mathrm{wt}\right)\end{array}$ & $\begin{array}{c}\text { Richness } \\
\text { (taxa/sample) }\end{array}$ & $\begin{array}{c}\text { EPT }^{b} \\
\text { Richness } \\
\text { (taxa/sample) }\end{array}$ & $\begin{array}{l}\text { Diversity } \\
\left(\mathrm{H}^{\prime}\right)\end{array}$ \\
\hline \multicolumn{6}{|l|}{ MIK 1.43} \\
\hline Year 1 & $88.7 \pm 26.9$ & $138.1 \pm 38.9^{d}$ & $20.1 \pm 3.6^{c}$ & $3.8 \pm 0.7$ & $3.5 \pm 0.3^{d}$ \\
\hline Year 2 & $298.5 \pm 63.5$ & $267.6 \pm 70.7$ & $32.3 \pm 3.0$ & $5.6 \pm 0.7$ & $3.8 \pm 0.1$ \\
\hline \multicolumn{6}{|l|}{ MIK 0.86} \\
\hline Year 1 & $34.9 \pm 13.4$ & $310.6 \pm 165.0^{\circ}$ & $5.7 \pm 1.1$ & $0.2 \pm 0.1$ & $1.6 \pm 0.1$ \\
\hline Year 2 & $28.2 \pm 5.8$ & $30.5 \pm 12.0$ & $7.4 \pm 1.0$ & $0.4 \pm 0.2$ & $2.0 \pm 0.2$ \\
\hline \multicolumn{6}{|l|}{ MIK 0.78} \\
\hline Year 1 & $30.6 \pm 8.2$ & $21.5 \pm 6.0$ & $5.4 \pm 1.0$ & $0.3 \pm 0.1$ & $1.5 \pm 0.2$ \\
\hline Year 2 & $46.6 \pm 22.1$ & $18.7 \pm 7.9$ & $6.3 \pm 1.2$ & 0 & $1.6 \pm 0.3$ \\
\hline \multicolumn{6}{|l|}{ MIK 0.71} \\
\hline Year 1 & $1.1 \pm 0.3^{\prime}$ & $2.3 \pm 1.4$ & $0.6 \pm 0.1^{c}$ & 0 & $0^{c}$ \\
\hline Year 2 & $10.5 \pm 4.5$ & $2.8 \pm 1.4$ & $2.3 \pm 0.5$ & 0 & $0.8 \pm 0.3$ \\
\hline \multicolumn{6}{|l|}{ MIK 0.54} \\
\hline Year 1 & $30.9 \pm 12.9^{d}$ & $37.0 \pm 18.6$ & $2.2 \pm 0.4^{c}$ & 0 & $0.7 \pm 0.2$ \\
\hline Year 2 & $105.8 \pm 48.1$ & $69.8 \pm 16.6$ & $6.6 \pm 1.6$ & 0 & $1.4 \pm 0.3$ \\
\hline \multicolumn{6}{|l|}{ MIK 0.45} \\
\hline Year 1 & $21.2 \pm 7.4^{c}$ & $89.1 \pm 38.7^{d}$ & $1.9 \pm 0.2^{c}$ & $0.1 \pm 0.1$ & $0.5 \pm 0.1$ \\
\hline Year 2 & $270.2 \pm 57.0$ & $146.0 \pm 47.3$ & $7.2 \pm 1.1$ & $0.1 \pm 0.1$ & $0.6 \pm 0.1$ \\
\hline
\end{tabular}

${ }^{a}$ Values are the means $\pm 1 \mathrm{SE}$, with $n=18$

${ }^{b}$ Ephemeroptera, Plecoptera, and Trichoptera.

Significant between-year difference, $p<0.0001$.

dSignificant between-year difference, $p<0.05$.

Significant between-year difference, $p<0.01$.

Significant between-year difference, $p<0.001$.

Note: MIK = Mitchell Branch kilometer.

site except for MIK 0.45 (Table 6.2).

Although biomass at MIK 0.45 did not differ statistically from biomass at MIK 1.43, the biomass at MIK 1.43 was almost two times greater. MIK 0.71 exhibited the greatest difference in density and biomass from MIK 1.43; at MIK 0.71 density and biomass were about 28 times and 96 times lower respectively. Densities at MIKs 0.54 and 0.45 were considerably higher than at MIKs 0.86 and 0.78 ; however, only the density at MIK 0.45 was significantly higher than at MIKs 0.86 and 0.78 . 
Table 6.2. Statistical comparisons of mean benthic macroinvertebrate density, biomass, taxonomic richness (number of taxa), EPT richness, and taxonomic diversity for Mitchell Branch, August 1987-January 1988

Parameter/site

Density

MIK 1.43 MIK 0.45 MIK 0.54 $\quad$ MIK 0.78 $\quad$ MIK $0.86 \quad$ MIK 0.71

Biomass

$\begin{array}{lllll}\text { MIK 1.43 } & \text { MIK 0.45 } & \text { MIK 0.54 } & \text { MIK 0.86 } & \text { MIK 0.78 }\end{array}$

Richness

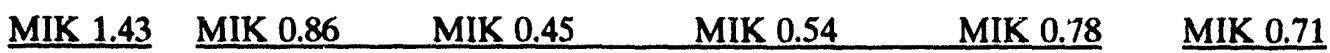

EPT richness ${ }^{b}$

$\begin{array}{lll}\text { MIK 1.43 } & \text { MIK } 0.86 & \text { MIK } 0.45\end{array}$

Diversity

\begin{tabular}{llllll} 
MIK 1.43 & MIK 0.86 & MIK 0.78 & MIK 0.54 & MIK 0.71 & MIK 0.45 \\
\hline
\end{tabular}

aSites are arranged in order from highest to lowest values from left to right. Sites not joined by lines are significantly different $(p<0.05)$, based on Tukey's studentized range test (HSD). $n=18$ for each site.

'No EPT taxa were collected from MIKs $0.78,0.71$, and 0.54 in quantitative samples.

Density and biomass varied considerably between sampling periods at most sites, particularly MIKs $1.43,0.45$, and 0.54 (Figs. 6.1 and 6.2). Few similarities in patterns of temporal change were noted among sites; this condition reflected between-site differences in taxonomic composition and community structure.
Between-year (August through January only) comparisons within each site revealed that, with few exceptions, values for density and biomass were higher during the second year than in the first year of BMAP (Table 6.1). These differences were statistically significant at all sites, except for density at MIKs 0.86 and 0.78 

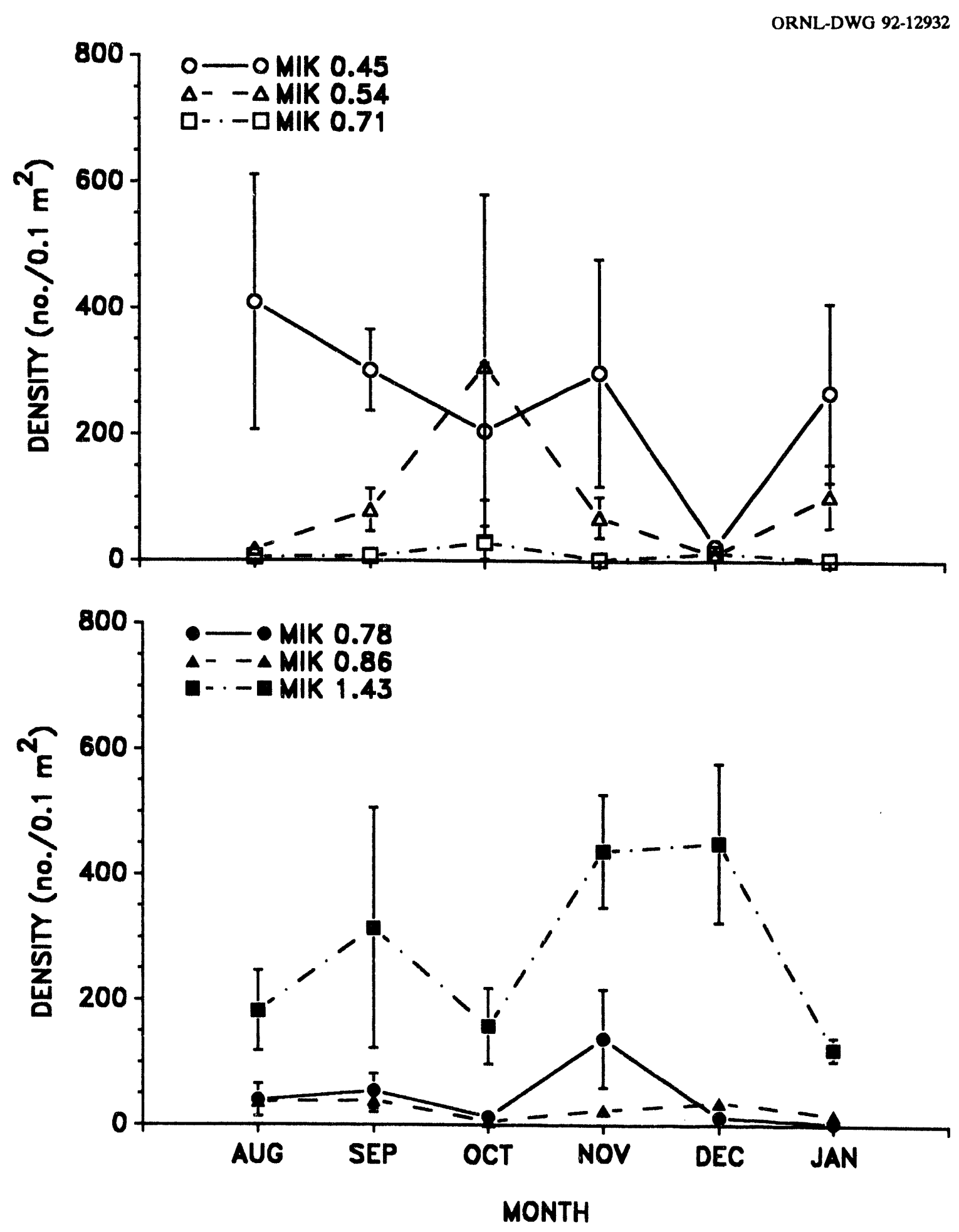

Fig. 6.1. Mean monthly density of benthic macroinvertebrates in Mitchell Branch. August 1987-January 1988. Values are the mean $\pm 1 \mathrm{SE}$. 

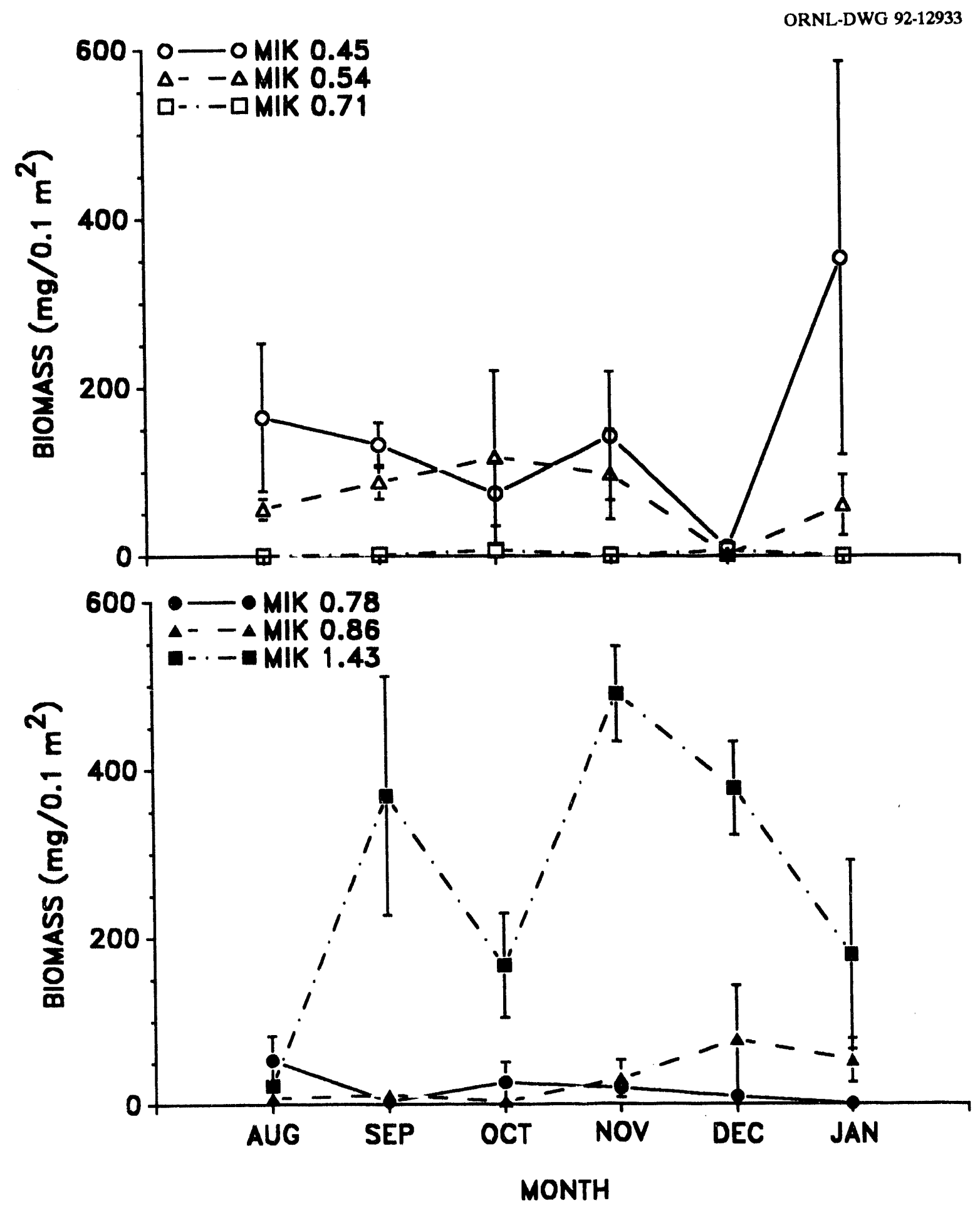

Fig. 6.2. Mean monthly biomass of benthic macroinvertebrates in Mitchell Branch, August 1987-January 1988. Values are the mean \pm 1 SE. 
and biomass at MIKs $0.78,0.71$, and 0.54 (Table 6.1). The most dramatic change in biomass occurred at MIK 0.86; the most dramatic change in density occurred at MIK 0.45. Biomass can be greatly influenced by the occasional collection of a few large taxa such as Decapoda (crayfish) and Tipulidae (Diptera: craneflies). The significant reduction in biomass at MIK 0.86 during the second year, with only a minor change in density, shows that large taxa were either absent or collected infrequently. The large increase in density at MIK 0.45 , coupled with a slight change in biomass, was probably the result of (1) higher densities of small taxa (e.g., oligochaetes and chironomids) that add little to the biomass and (2) the infrequent occurrence of larger taxa (e.g., Decapoda and Tipulidae).

\subsubsection{Dominant taxa*}

Considerable spatial and temporal differences were observed in taxonomic composition of the benthic community in Mitchell Branch. Insight into the possible reasons for these differences may be gained from considering the dominant taxonomic groups because these taxa usually account for most of the betweensite differences.

The most dominant taxa at all sites in Mitchell Branch were oligochaetes and chironomids; these two taxa combined for more than $80 \%$ of the total density at all sites, except for MIKs 1.43 and 0.78 , where they accounted for more than $60 \%$ of the total density (Table 6.3). Oligochaetes

\footnotetext{
*For the benthic macroinvertebrate section of this report, the term dominant is used synonymously with numerical dominance. Taxa were considered numerically dominant if they were collected at $50 \%$ or more of the study sites and comprised $\geq 10 \%$ of the average density at two or more sites.
}

became more dominant with distance downstream from MIK 1.43, whereas the percentage of chironomids tended to decrease with distance downstream from MIK 1.43. In addition to their numerical dominance, these two groups also contributed considerably to the total biomass at all sites; their combined contribution to biomass was greater than $40 \%$ at all sites except for MIK 1.43.

Other taxa also contributed substantially to either the density or biomass of the invertebrate community at some sites, or some taxa [e.g., Ephemeroptera, Plecoptera, and Trichoptera (EPT) taxa] provided useful information for assessing the water quality of the stream because of their overall sensitivity to changes. Dipterans (excluding Chironomidae) were moderately abundant at most sites, accounting for up to $7.9 \%$ of the total density and up to $22.2 \%$ of the total biomass (Table 6.3). EPT taxa comprised a substantial part of the total density and/or biomass at MIK 1.43 only (Table 6.3). These taxa were not collected in quantitative samples from MIKs 0.78 , 0.71 , or 0.54 , and they contributed very little to either density or biomass at MIKs 0.86 and 0.45 .

\subsubsection{Community structure}

Taxonomic richness. Spatial patterns in taxonomic richness were similar to those observed for density and biomass (Table 6.1). The site having the greatest number of taxa per sample was MIK 1.43, where richness was more than four times greater than at any of the other sites. The site with the fewest number of taxa per sample was MIK 0.71 , where there was a 15-fold difference from that for MIK 1.43. Richness at the remaining four sites was similar and statistically indistinguishable, whereas richness at MIKs 1.43 and 0.71 was significantly greater and less, 

Table 6.3. Relative density (mean percentage \pm 1 SE) and biomass (mean percentage \pm 1 SE) of selected macroinvertebrate
tar in Mitchell Branch, Angust 1987-January 1988

\begin{tabular}{|c|c|c|c|c|c|c|}
\hline \multirow[b]{2}{*}{ Taxon } & \multicolumn{6}{|c|}{ Site $^{a}$} \\
\hline & $\begin{array}{c}\text { MIK } \\
1.43\end{array}$ & $\begin{array}{l}\text { MIK } \\
0.86\end{array}$ & $\begin{array}{l}\text { MIK } \\
0.78\end{array}$ & $\begin{array}{l}\text { MIK } \\
0.71\end{array}$ & $\begin{array}{l}\text { MIK } \\
0.54\end{array}$ & $\begin{array}{l}\text { MIK } \\
0.45\end{array}$ \\
\hline $\begin{array}{c}\text { Oligochaeta } \\
\text { Density } \\
\text { Biomass }\end{array}$ & $\begin{array}{l}14.8 \pm 3.7 \\
3.4 \pm 0.7\end{array}$ & $\begin{array}{l}23.5 \pm 8.4 \\
18.3 \pm 9.1\end{array}$ & $\begin{array}{l}33.8 \pm 10.6 \\
32.6 \pm 14.0\end{array}$ & $\begin{array}{l}46.4 \pm 13.6 \\
39.5 \pm 12.0\end{array}$ & $\begin{array}{l}59.4 \pm 8.5 \\
62.9 \pm 6.7\end{array}$ & $\begin{array}{l}89.3 \pm 2.6 \\
85.2 \pm 3.9\end{array}$ \\
\hline $\begin{array}{l}\text { Chironomida } \\
\text { Density } \\
\text { Biomass }\end{array}$ & $\begin{array}{c}48.0 \pm 7.1 \\
8.9 \pm 4.9\end{array}$ & $\begin{array}{c}60.6 \pm 11.3 \\
25.1 \pm 7.5\end{array}$ & $\begin{array}{l}32.3 \pm 11.4 \\
16.1 \pm 11.6\end{array}$ & $\begin{array}{l}35.9 \pm 14.7 \\
35.1 \pm 13.9\end{array}$ & $\begin{array}{c}23.3 \pm 8.1 \\
7.6 \pm 4.8\end{array}$ & $\begin{array}{l}6.4 \pm 2.7 \\
2.1 \pm 1.0\end{array}$ \\
\hline $\begin{array}{l}\text { Diptera }^{b} \\
\text { Density } \\
\text { Biomass }\end{array}$ & $\begin{array}{c}4.6 \pm 0.6 \\
12.6 \pm 6.1\end{array}$ & $\begin{array}{c}5.9 \pm 1.7 \\
22.2 \pm 9.9\end{array}$ & $\begin{array}{l}2.4 \pm 1.1 \\
5.3 \pm 3.2\end{array}$ & $\begin{array}{r}7.9 \pm 4.2 \\
13.9 \pm 8.0\end{array}$ & $\begin{array}{l}6.3 \pm 2.5 \\
7.5 \pm 3.5\end{array}$ & $\begin{array}{l}1.8 \pm 0.7 \\
0.7 \pm 0.3\end{array}$ \\
\hline $\begin{array}{c}\text { Ephemeropt } \\
\text { Density } \\
\text { Biomass }\end{array}$ & $\begin{array}{l}6.0 \pm 1.0 \\
4.0 \pm 1.2\end{array}$ & $\begin{array}{l}1.2 \pm 0.8 \\
1.8 \pm 1.6\end{array}$ & $\begin{array}{l}0 \\
0\end{array}$ & $\begin{array}{l}0 \\
0\end{array}$ & $\begin{array}{l}0 \\
0\end{array}$ & $\begin{array}{l}0 \\
0\end{array}$ \\
\hline $\begin{array}{c}\text { Plecoptera } \\
\text { Density } \\
\text { Biomass }\end{array}$ & $\begin{array}{c}12.4 \pm 5.5 \\
5.0 \pm 2.2\end{array}$ & $\begin{array}{l}0.4 \pm 0.2 \\
3.4 \pm 3.4\end{array}$ & $\begin{array}{l}0 \\
0\end{array}$ & $\begin{array}{l}0 \\
0\end{array}$ & $\begin{array}{l}0 \\
0\end{array}$ & $\begin{array}{l}\mathbf{0} \\
\mathbf{0}\end{array}$ \\
\hline $\begin{array}{c}\text { Trichoptera } \\
\text { Density } \\
\text { Biomass }\end{array}$ & $\begin{array}{c}1.6 \pm 0.6 \\
14.2 \pm 5.6\end{array}$ & $\begin{array}{c}0.1 \pm 0.1 \\
<0.1\end{array}$ & $\begin{array}{l}0 \\
0\end{array}$ & $\begin{array}{l}\mathbf{0} \\
0\end{array}$ & $\begin{array}{l}0 \\
0\end{array}$ & $\begin{array}{l}<0.1 \\
<0.1\end{array}$ \\
\hline
\end{tabular}

-MIK = Mitchell Branch kilometer.

'Excludes Chironomidae. 
respectively, than at all other sites (Table 6.2).

Most of the sites exhibited temporal patterns in richness similar to those of density and biomass, with few similarities between sites (Fig. 6.3). One striking difference between MIK 1.43 and the downstream sites was that richness values of less than 20 taxa per sample were never observed at MIK 1.43 , whereas at the remaining 5 sites, richness never exceeded 12 taxa per sample during any sampling period and was usually less than 10 taxa per sample. Furthermore, variability
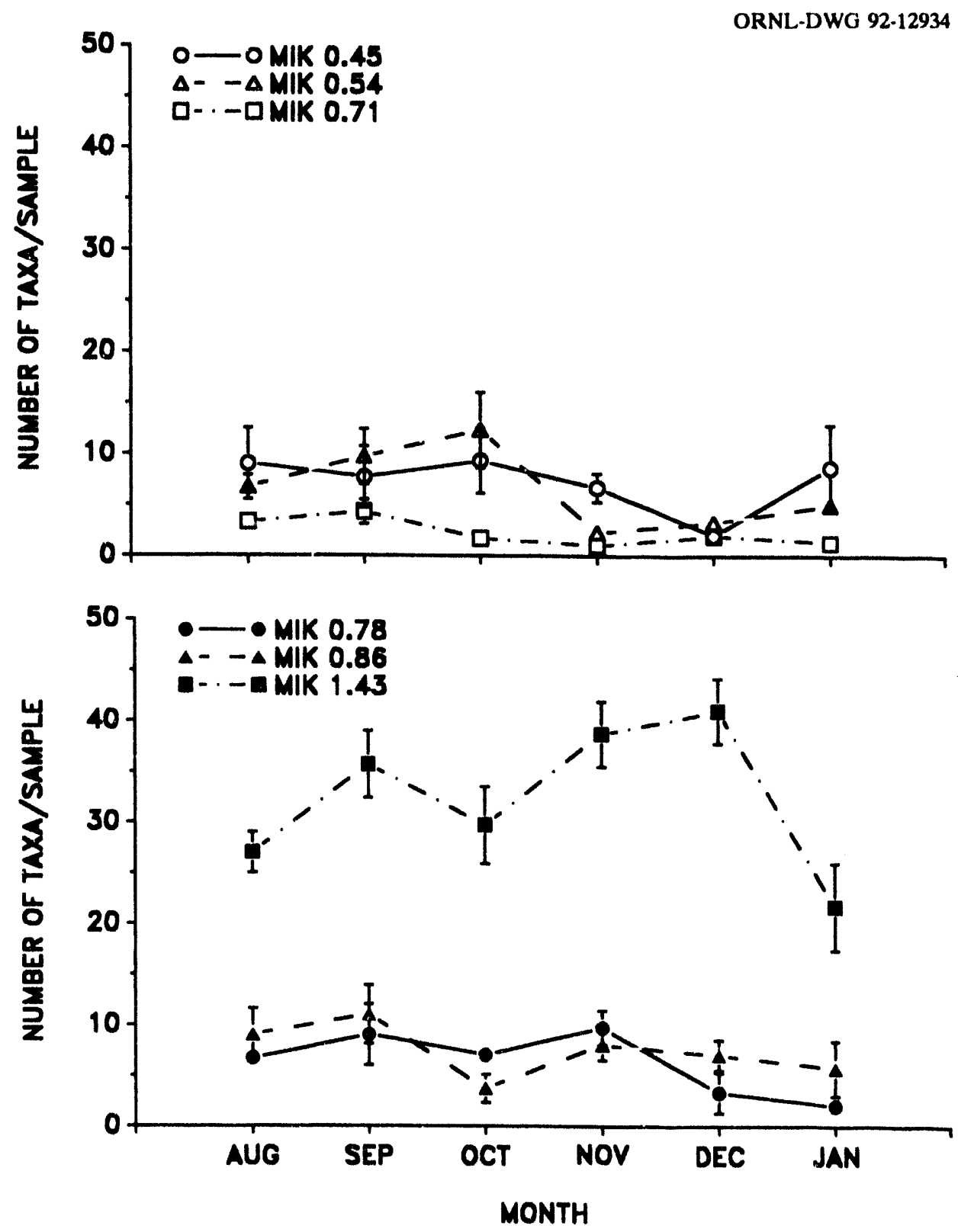

Fig. 6.3. Mean monthly taxonomic richness of benthic macroinvertebrates in Mitchell Branch, August 1987-January 1988. Values are the mean $\pm 1 \mathrm{SE}$. 
between replicate samples was generally much greater at MIK 1.43 than at the other sites during each sampling period, which may reflect a more complex community structure at this site (i.e., variability between replicates should decrease as the number of taxa that can potentially be collected decreases).

Comparisons between years showed that richness values at all sites were higher during the second year of the monitoring program then they were during the first year (Table 6.1). These differences were very highly significant $(p<0.0001)$ at all sites except MIKs 0.86 and 0.78 , where the values did not differ significantly between years.
EPT richness. During the second year EPT taxa were not obtained in quantitative samples from MIKs $0.78,0.71$, and 0.54 (Table 6.1). EPT taxa were rare at MIKs 0.45 and 0.86 , where they were collected in only 2 and 4 of the 6 months, respectively (Fig. 6.4), while at MIK 1.43 the number of EPT taxa per sample was never less than three and exceeded five in 4 of 6 months. Statistical comparison of the three sites having EPT taxa showed that there were significantly more EPT taxa at MIK 1.43 than at the two downstream sites and that the number of EPT taxa at the two downstream sites did not differ (Table 6.2). Between-year differences were minimal in EPT richness at all

ORNL-DWG 92-12935

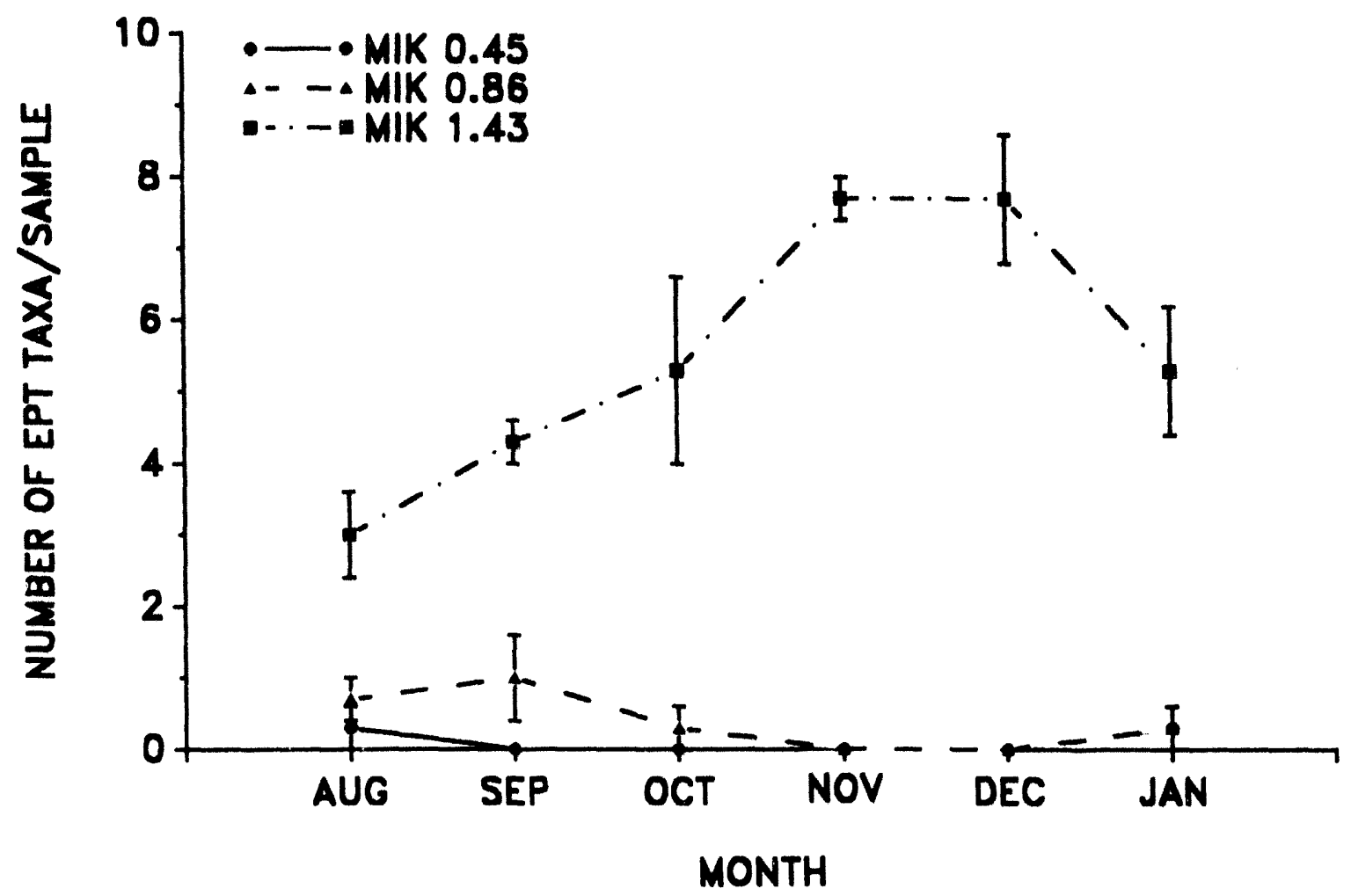

Fig. 6.4. Mean monthly richness of Ephemeroptera, Plecoptera, and Trichoptera (EPT richness) in Mitchell Branch, August 1987-January 1988. Values are the mean \pm 1 SE. 
sites except MIK 1.43, where the value for the second year was significantly greater than for the first year (Table 6.1).

Diversity. Spatial patterns in taxonomic diversity were similar to those observed for density, biomass, and taxonomic richness, with the highest value obtained from MIK 1.43 and the lower values from the downstream sites (Tables 6.1 and 6.2). Diversity was significantly greater at MIK 1.43 than at all other sites, whereas differences in diversity at MIKs $0.86,0.78$, and 0.54 were not significant. Unlike the other parameters, the lowest mean value for diversity was found at MIK 0.45; however, mean diversity at this site did not differ significantly from mean diversity at MIK 0.71 .

Temporally, MIK 1.43 exhibited less dramatic fluctuations in diversity than the other sites, and never fell below $\mathbf{3 . 0}$

(Fig. 6.5). At the other sites, however, diversity exhibited more dramatic monthly fluctuations and generally remained at $\mathbf{2 . 0}$ or less. All sites exhibited increases in diversity between years, but the increases were significant at only MIKs 1.43 and 0.54 (Table 6.1).

\subsubsection{Discussion}

The benthic invertebrate community of Mitchell Branch within the boundaries of the Oak Ridge K-25 Site continued to show evidence of severely degraded water quality during the first 6 months of the second year of BMAP. Relative to the reference site in upper Mitchell Branch (MIK 1.43), density, biomass, taxonomic richness, EPT richness, and taxonomic diversity were all substantially lower at the five downstream sites. The most notable differences between MIK 1.43 and the downstream sites were in taxonomic and EPT richness. Taxonomic richness was at least four times higher at MIK 1.43 than at any of the other sites, and EPT taxa, which generally tend to be sensitive to changes in water quality (Wiederholm 1984), were either absent or very rarely collected at the sites downstream of MIK 1.43.

As was found in the first year of BMAP (Smith et al. 1993), the most severely affected site in Mitchell Branch was MIK 0.71. Values for density, biomass, taxonomic richness, and EPT richness were all significantly lower at this site than at all other sites. Also, no EPT taxa were collected from this site, and the number of taxa collected per sample (richness) was at least 2.7 times less than at any of the other sites.

The extent of impact on the benthic invertebrate community at the remaining four affected sites (MIKs 0.86, 0.78, 0.54, and 0.45 ) was somewhat less than at MIK 0.71. Although differences in the invertebrate community existed between thuse four sites (e.g., differences in relative density and biomass of the dominant taxa), differences in the extent of impact were not readily discernable. Relative to other affected stre .,ns on the ORR, the benthic community at these sites clearly reflected highly stressed conditions. For example, in EFPC (Smith 1992a) and in streams in the White Oak Creek watershed (Smith 1992b), the most highly stressed benthic communities are usually made up almost exclusively of chironomids and/or oligochaetes, whereas less-pollutiontolerant taxa such as Ephemeroptera and Trichoptera are rare or absent. As water quality improves in these streams with distance downstream of effluent discharges, the relative abundance of the chironomids and oligochaetes decreases as the relative abundance of Ephemeroptera and Trichoptera increases. In relatively unaffected sites, the latter two taxonomic groups and Plecoptera generally contribute substantially to the total density. In Mitchell Branch, EPT taxa were rare or absent from all sites except for MIK 1.43, and the chironomids and oligochaetes 
ORNL-DWG 92-12936
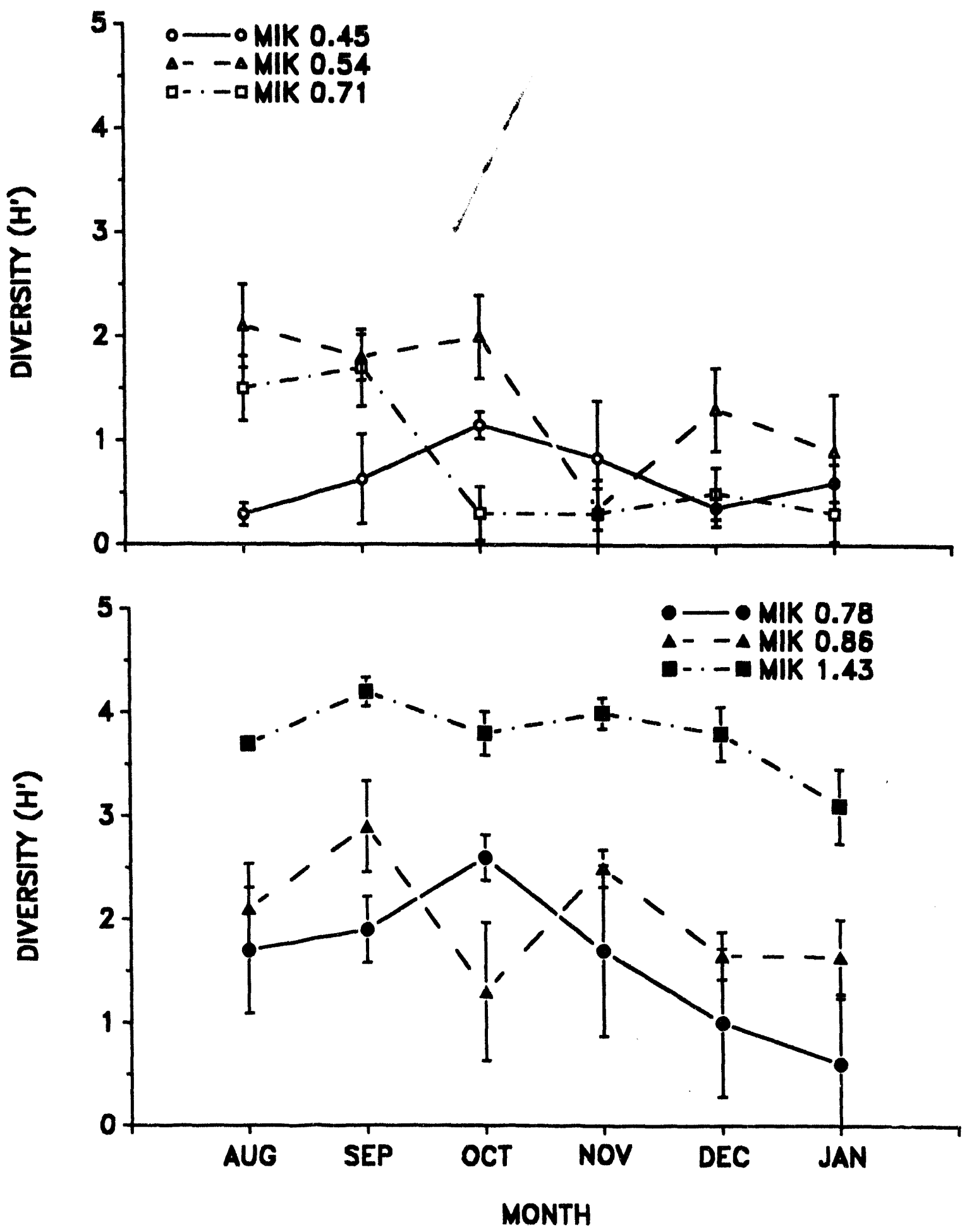

Fig. 6.5. Mean monthly taxonomic diversity $\left(\mathrm{H}^{\prime}\right)$ of benthic macroinvertebrates in Mitchell Branch, August 1987-January 1988. Values are the mean \pm 1 SE. 
accounted for over $65 \%$ of the total density at all affected sites.

Between-year changes in the benthic community of each site were examined for trends indicative of recovery. Most sites exhibited increases in density, biomass, taxonomic richness, and taxonomic diversity, and in many cases, these increases were statistically significant. EPT richness, on the other hand, changed little between years at any site except for MIK 1.43, where the value was significantly higher during the second year.

Benthic invertebrate communities exhibit natural year-to-year changes in composition, richness, relative abundance of taxa, etc. (e.g., McElravy et al. 1989). However, numerous factors including the biota present, the location and types of effluent discharges, the extent and type of streamside vegetation, substrate composition, and other abiotic factors make it unlikely that even sites as close to each other as those in Mitchell Branch would exhibit similar changes between years (e.g., substantial increases at most sites in density and taxonomic richness). Thus, the magnitude of most of the observed between-year changes in Mitchell Branch was probably not the result of natural variability alone, but to other factors, such as improving or worsening conditions, and/or between-year differences in laboratory sample processing efficiency (i.e., the percentage of organisms removed from a sample). A preliminary analysis of some quality assurance checks of sample processing efficiency of JAYCOR personnel indicated that processing efficiency has improved from consistently $<70 \%$ by the Tennessee Valley Authority, the former processing subcontractor, to consistently $>90 \%$ by JAYCOR, the current processing subcontractor (J. G. Smith, ESD, ORNL, unpublished data). Thus, at least some of the between-year differences observed within each site may have been the result of differences in sample processing efficiency.

Even though processing efficiency of the samples differed between years, this difference did not appear to have much effect on distinguishing spatial trends (i.e., similar spatial trends were cvident in Mitchell Branch during both years). However, differences in processing efficiency did reduce the ability to detect betweenyear differences within sites, which reduced the ability to detect possible recovery. In an attempt to identify changes that may be indicative of recovery, the data for each affected site in each year were first "normalized" to the reference site by comparing each parameter value (i.e., density, biomass, taxonomic richness, and EPT richness) from each affected site with that of MIK 1.43 and then dividing the largest of the two values (i.e., affected vs reference site) by the smallest value to obtain a difference factor between the sites. For illustration, the resulting values were designated as negative an the parameter value was highest at the affected site and positive when the parameter value was highest at the reference site. Assuming that (1) processing efficiency had no effect on the magnitude of difference between sites within each year and (2) the reference site remained relatively undisturbed, the magnitude of change between years was then determined for each parameter by subtracting the difference factor for the second year (August 1987-January 1988) from the difference factor for the first year (August 1986-January 1987).

Thus, for a site that exhibited no between-year change relative to its reference site, the resulting value was zero, whereas a positive or negative change between years could be indicative of improving or worsening conditions respectively. : or example, in the first year mean density at MIKs 0.86 and 1.43 , the reference site, was 34.9 and 88.7 individuals per 
$0.1 \mathrm{~m}^{2}$, respectively, giving a difference factor of 2.5. In the second year, mean densities at these sites were 28.2 and 298.5 individuals per $0.1 \mathrm{~m}^{2}$, respectively, giving a difference factor of $\sim 10.6$. The magnitude of change between the two years in density at MIK 0.86 would then be the first year difference factor (2.5) minus the second year difference factor (10.6), which would equal -8.1. This value would indicate that, relative to the reference site, density declined at MIK 0.86 during the second year, which may be indicative of worsening conditions.

As would be expected under natural conditions, between-year changes occurred in most parameters at most sites (Fig. 6.6). Differences in parameter values between the reference site and MIKs 0.86 and 0.78 tended to be greater during the second than the first year, whereas the trend was generally just the opposite at MIKs 0.54 and 0.45 . However, the magnitude of between-year changes at these four affected sites tended to be minor (i.e., magnitude of change $<10$ ). Thus, the data suggest that these sites changed little between years and that these changes could be, at least in part, the results of natural variability. The most dramatic between-year changes occurred at MIK 0.71. This site showed substantial increases in both density and taxonomic richness (magnitude of change $>20$ ) and a substantial decline in biomass (magnitude of change of 35.6). Although the magnitude of change in density and taxonomic richness implies that improvements may have occurred at this site since the first year of BMAP, records on sample processing efficiency do not support this. Records on processing efficiency of samples indicated that in most cases lower efficiency was associated with samples having low numbers of organisms $(<100)$ (J. G. Smith, ESD, ORNL, unpublished data). This was probably because most of the organisms collected at this site are very small (i.e., chironomids) and thus more easily overlooked. For a site such as MIK 0.71, where very low numbers of organisms were collected ( $\sim 10$ individuals per sample), differences in sample processing efficiency could result in substantially greater magnitudes of change between years than for a site having much higher numbers of organisms.

No substantial changes were evident in the benthic community between the first 6 months of the first and second years of BMAP. This finding indicates that neither significant improvements nor further degradation have occurred in Mitchell Branch during this period. As hypothesized in the first report on Mitchell Branch, siltation is probably one of the main perturbations, particularly at MIKs 0.86 and 0.78 , which are upstream of all major effluent discharges. Construction activities along Mitchell Branch have resulted in the removal of riparian vegetation and the exposure of surface soils, both of which can contribute to erosion and thus increase the silt load in the stream. Removal of riparian vegetation also increases the rate of surface water runoff, which in turn affects stream discharge during periods of rain and further reduces habitat stability within the stream. A substratum dominated by mud and silt tends to favor oligochaetes (Whitley 1982). The substratum in Mitchell Branch becomes increasingly covered with and/or dominated by silt with increasing distance from the headwaters, which may partially explain why oligochaetes become increasingly dominant with distance downstream.

Although siltation appears to have an important impact on the lower three sites in Mitchell Branch, the structure and composition of the benthic invertebrate community indicate the presence of toxicants in addition. This was particularly evident at MIK 0.71, where total density, biomass, and taxonomic richness were all very low, a condition characteristic of 


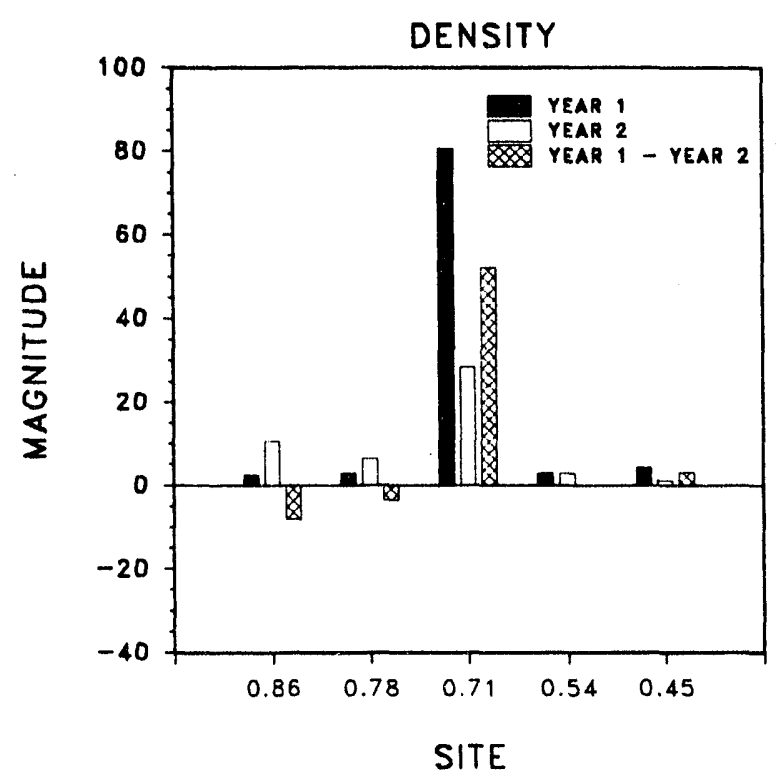

ORNL-DWG 92-12937
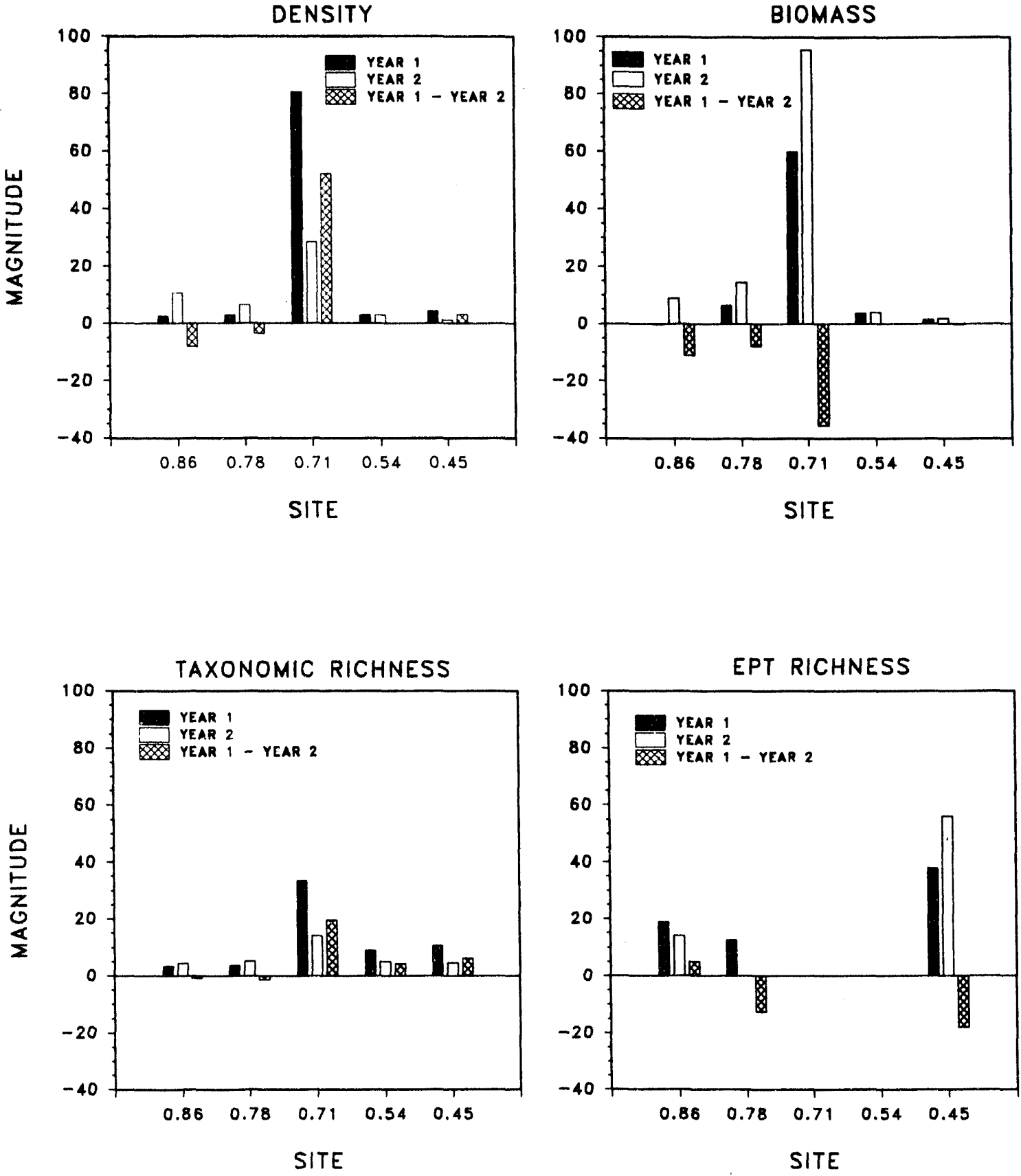

Fig. 6.6. Difference factor between each downstream site and the reference site (Mitchell Branch kilometer 1.43) (Year 1, August 1986-January 1987; Year 2, August 1987-January 1988) and the magnitude of change within each site between Year 1 and Year 2 (crosshatched bars) for mean density, biomass, taxonomic richness, and richness of Ephemeroptera, Plecoptera, and Trichoptera (EPT richness) in Mitchell Branch. 
benthic communities exposed to toxicants (e.g., Wiederholm 1984). Based on the results obtained in the toxicity monitoring program (Sect. 3; see also Sect. 3 in Smith et al. 1993), chlorine is the most likely toxicant affecting the invertebrate community at these sites, originating primarily from SD 170. The dominance of taxa that are capable of producing several generations per year (i.e., oligochaetes and chironomids), suggests that the benthic community at these sites is probably exposed intermittently to the toxicant(s). These taxa probably have resistent (e.g., egg) or unexposed (i.e., terrestrial adult) stages in the stream at most times. Thus, between toxic releases such organisms are capable of rapid recolonization. This "intermittent release" hypothesis is supported by water quality data collected concurrently with the toxicity monitoring program, which found that high concentrations of chlorine occurred periodically (Sect. 3.2.3).

Other factors (e.g., elevated temperatures, flow augmentation, and elevated concentrations of metals and organics) identified and discussed in the first report continue to be potential perturbations (Smith et al. 1993), and reductions in Ceriodaphnia fecundity at all sites downstream of MIK 1.0 provide evidence of chronic toxicity (Sect. 3.2). However, it is not currently possible to determine the extent of influence of these factors from the available data.

\subsubsection{Conclusions}

The structure and composition of the benthic invertebrate community in Mitchell Branch within the boundary of the Oak Ridge K-25 Site continue to indicate that water quality in this portion of the stream is severely degraded. Maximum impact occurs just downstream of SD 170, but within a short distance minor improve- ment is evident; thus, SD 170 clearly continues to be the major source of impact.

Several factors are probably involved in the degradation of the invertebrate community, but the two most important factors appear to be silt and the presence of one or more toxicants (e.g., chlorine). Excessive quantities of silt were evident at all sites and may have been the major cause of impact to the invertebrate community upstream of SD 170. Silt also appears to be an important cause of impact to the invertebrate community downstream of SD 170, but the presence of one or more toxicants also appears to be affecting this portion of the stream.

\subsubsection{Future Studies}

The initial benthic invertebrate monitoring program in which the sampling frequency was monthly was completed in July 1988; the sampling frequency was then reduced to quarterly. Qualitative samples will continue to be collected annually during the spring of each year at the nonreference sites, but a qualitative sample will no longer be collected from the reference site unless the site shows evidence of impact. Data analyses in future reports will continue to incorporate information obtained from other monitoring programs on the ORR where appropriate, and efforts to obtain more sensitive parameters to help ascertain the status of the benthic community, such as EPT richness, will also continue.

\subsection{FISHES (M. G. Ryon)}

\subsubsection{Introduction}

Fish population and community studies can be used to assess the ecological effects of water quality and habitat. These 
studies offer several advantages over other indicators of environmental quality (see Fausch et al. 1990, Karr et al. 1986, Karr 1987, Karr 1991) and are relevant to any evaluation of the biotic integrity of streams such as Mitchell Branch. Fish communities, for example, comprise several trophic levels, with species that are at or near the end of food chains. Consequently, they integrate the direct effects of water quality and habitat changes on primary producers (periphyton) and consumers (benthic invertebrates) that are used for food. Because of these trophic interrelationships, the well-being of fish populations has often been used as an index of water quality (e.g., Weber 1973; Greeson et al. 1977; Karr et al. 1991). Moreover, statements about the condition of the fish community are understood by the general public (Karr 1981).

The initial objectives of the instream fish monitoring task (Subtask 4b of BMAP, as described in Loar et al. 1991) were to (1) characterize spatial and temporal patterns in distribution and abundance of fishes in Mitchell Branch and (2) document possible effects on fish community structure and function resulting from implementation of the K-25 Site Water Pollution Control Program.

\subsubsection{Methods}

The fish community in Mitchell Branch was evaluated at five sites (Fig. 2.1 and Table 2.8), four of which reflect reaches potentially affected by the Oak Ridge K-25 Site effluents (Sect. 2.2.3). The most upstream site, MIK 1.43, served as an unaffected reference. An additional, nearby reference site (GCK 2.4) was sampled also (Fig. 2.2). The affected Mitchell Branch sites were sampled four times during 1988, twice in 1989, and once in the spring of 1990 (Table 6.4). Grassy Creek was sampled three times during
1988 , twice in 1989 , and once in the spring of 1990 . The other reference site, MIK 1.43, was dropped from the sampling program in the fall of 1988 because the shallow water did not provide fish habitat similar to the downstream sites (Table 6.4). The absence of similar habitat made fish community comparisons invalid.

All sampling was conducted with the use of a Smith-Root Model 15A backpack electrofisher. The unit has a self-contained, gasoline-powered generator capable of delivering up to $1200 \mathrm{~V}$ of pulsed direct current. The pulse frequency and the output voltage can be varied, but generally a pulse frequency of 90 to $120 \mathrm{~Hz}$ and a voltage of less than $400 \mathrm{~V}$ was used. The circular (ring) electrode at the end of the fiberglass anode pole was fitted with a nylon net $(0.64-\mathrm{cm}$ mesh) to allow the operator to assist in collecting stunned fish.

After 0.64-cm-mesh seines were placed across the upper and lower boundaries of the fish sampling site to restrict fish movement, a two- to three-person sampling team electrofished through the site in an upstream direction, making three consecutive passes. Stunned fish were collected and separated by pass in buckets prior to processing. If the number of fish captured during the first pass was extremely low or zero, only one pass was made. Depending on the turbidity of the water, the consecutive passes could not always be made immediately. Rather, fish were processed after each pass to allow sufficient time for the water to clear before another pass was started.

Following the electrofishing, fish were anesthetized with MS-222 (tricaine methanesulfonate), identified, measured to the nearest $0.1 \mathrm{~cm}$ (total length), and weighed to the nearest $0.1 \mathrm{~g}$ (for fish less than $100 \mathrm{~g}$ ) or $1 \mathrm{~g}$ (for fish greater than $100 \mathrm{~g}$ ) by using Pesola spring scales. At sites with high fish densities, individuals were recorded by $1-\mathrm{cm}$ size classes and species. After 25 individuals of a 
Table 6.4. Length, mean width, mean depth, area, and pool/rifife ratio (P/R) of fish sampling sites on Mitchell Branch and Grassy Creek,

a reference stream, January 1988-May 1990

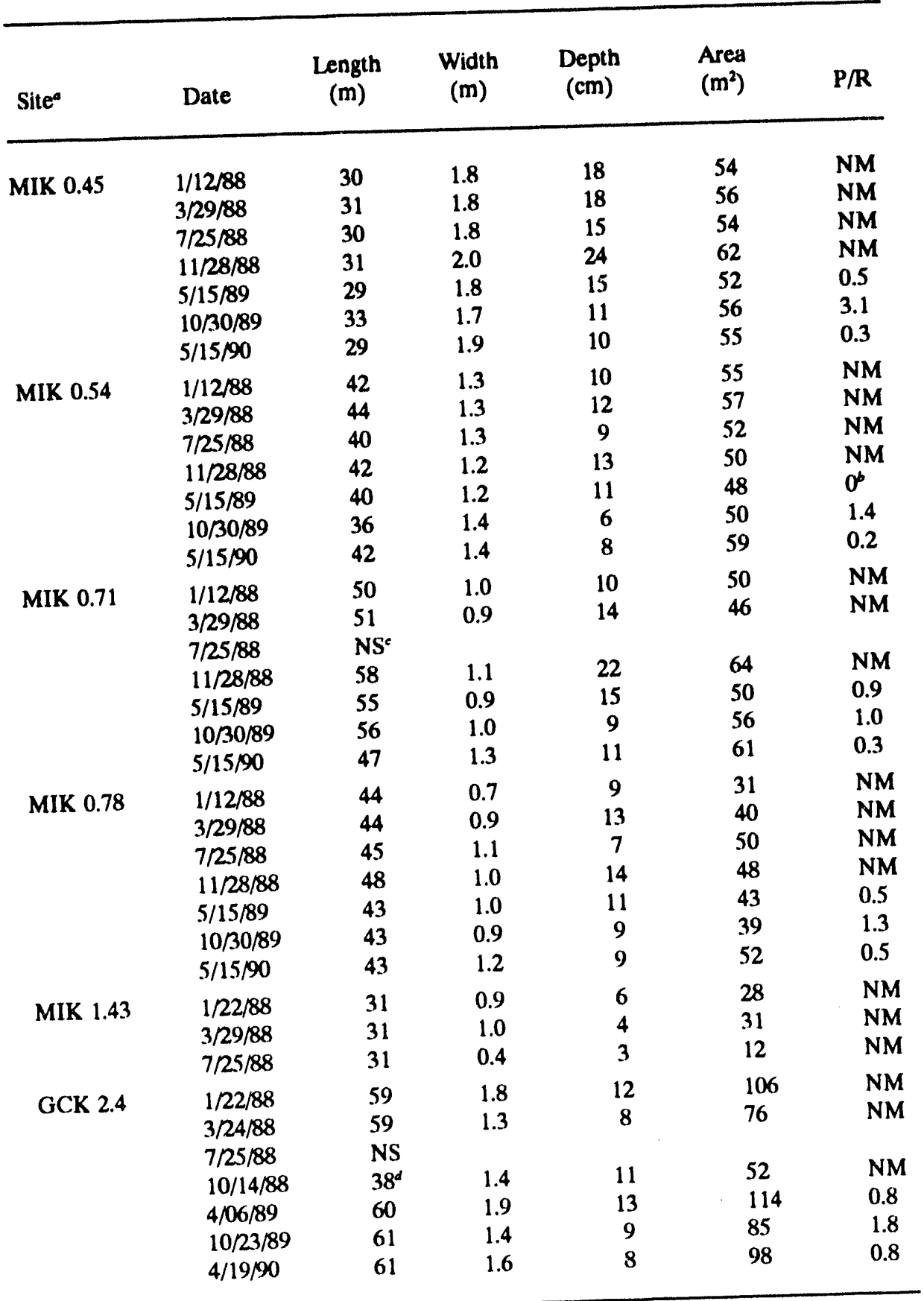

${ }^{a}$ MIK = Mitchell Branch kilometer; GCK $=$ Grassy Creek kilometer.

bStream section contained only pools.

'Site not sampled during this period.

${ }^{d}$ Length reflects only pools; riffle areas were dry and not measured.

Note: $\mathrm{NM}=$ not measured for this sample. 
species-size class were measured and weighed, additional members of that size class were only measured. Length-weight regressions were later used to estimate missing weight data (Railsback et al. 1989). Other data recorded (if possible to determine), included sex, reproductive state, disposition (i.e., dead or kept for laboratory identification and inclusion in a reference collection), and the presence of any abnormalities (e.g., external parasites, skeletal deformities, etc.). After processing fish from all passes, the fish were allowed to fully recover from the anesthetic and were returned to the stream within the sampling site. Any additional mortality occurring as a result of processing was noted at that time.

Also recorded were data on selected physical and chemical parameters of the stream site and on the sampling effort. A Horiba Model U-7 Water Quality Checker was used to measure $\mathrm{pH}$, temperature, dissolved oxygen, and conductivity. An HF Instruments Model DRT-15 turbidimeter was used to measure turbidity. The duration of the electrofishing effort was recorded, and a visual estimate was made of percentage cloud cover. Following completion of fish sampling, the length, widths (at 5-m intervals), and depths (at three points on the width transect) of the sampling reach were measured at each site.

Species population estimates were calculated by using the three-pass removal method of Carle and Strub (1978). Biomass was estimated by multiplying the population estimate by the mean weight per individual. Annual production was estimated at each site by using a sizefrequency method (Garman and Waters 1983). Total numbers, biomass, and production were divided by the surface area $\left(\mathrm{m}^{2}\right)$ of the sampling site to calculate density, biomass, and annual production per unit area. For each sampling date, surface area was estimated by multiplying the length of sampling reach by the mean width, based on measurements taken at 5-m intervals (Table 6.4). The procedures used for calculating density, biomass, and production are given in more detail in Railsback et al. (1989).

Condition factors measure the degree of plumpness of a fish as an index of relative health (Bennett 1970). A condition factor $(K)$ was calculated for individual fish according to the formula

$$
K=100\left(\text { weight/length }{ }^{3}\right),
$$

with weight in grams and total length in centimeters (Hile 1936). Fish weights estimated by regression were not used in the calculation of condition factors. Comparisons of condition factors between sites and between sampling periods were made with an ANOVA on untransformed data (PROC GLM, SAS 1985b) because the condition factors exhibited homogeneity of the variance (PROC UNIVARIATE, SAS 1985a). If the ANOVA indicated significant differences in condition factors between groups, the Tukey test was performed to identify those groups that were significantly different $(p<0.05)$.

\subsection{Results and Discussion}

Surveys made from 1988 to 1990 continued to show declines in fish population densities and biomass that were seen at most sites during the first year of BMAP (Ryon 1993), with the declines resulting in the absence of fish populations in much of Mitchell Branch. However, a slight increase in number of fish species was observed in 1990 in contrast to the earlier sampling period.

\subsubsection{Species composition and richness}

During the first 2 years of sampling (October 1986 to October 1993), the fish 
community of Mitchell Branch was a simple, three-species complex (Table 6.5). The complex consisted of the blacknose dace, Rhinichthys atratulus, the creek chub, Semotilus atromaculatus, and the redbreast sunfish, Lepomis auritus (Ryon 1993). All three species appear to be insensitive to many habitat and water quality stresses and would not be considered intolerant species in an impact assessment methodology such as the Index of Biotic Integrity (Karr et al. 1986). During the last two sampling periods, two additional species were collected in Mitchell Branch (Table 6.5). The central stoneroller, Campostoma anomalum, and the striped shiner, Luxilus chrysocephalus, were found in low numbers at MIK 0.78. In comparison with similar-sized, relatively undisturbed area streams, the fish species richness in Mitchell Branch was typical of that found in headwater streams (Ryon and Loar 1988). In upper Grassy Creek at GCK 2.4, the striped shiner, white sucker (Catostomus commersoni), banded sculpin (Cottus carolinae), and green sunfish (L. cyanellus) occurred with blacknose dace and creek chub during the same period. The faunal differences in these two streams may reflect temperature or water quality differences between the sites, although only the sculpin is considered to be an intolerant species (Karr et al. 1986).

\subsubsection{Population densities}

Surveys from 1988 to 1990 indicated that fish populations had disappeared from sites other than MIK 0.78 (Table 6.5). The only fish taken outside of this site was collected in the May 1990 sample at the upper end of MIK 0.71. This large creek chub may have been displaced from a section just upstream of SD 170 . The effluent from SD 170 enters Mitchell Branch in the lower reach of fish sampling site MIK 0.78. In the 1988 to 1990 surveys, no fish were collected in this site below where this effluent enters Mitchell Branch. This effluent is consistently toxic to fathead minnows in laboratory toxicity tests (Table 3.6). Episodic releases of chlorine (Sect. 3.2.3) may prevent fish from establishing permanent residency in the section of Mitchell Branch below the SD 170 outfall and above the embayment near Poplar Creek. The greatest impact on the benthic invertebrate community also occurred at this site (Table 6.2).

The 1988-90 surveys also documented declines in the fish population at MIK 0.78 above SD 170. In the first year's surveys (1986 to 1987), the population densities at MIK 0.78 averaged 3.2 fish per square meter (Ryon 1993). During 1988, the average population density dropped to 1.5 fish per square meter before increasing somewhat to 2.3 fish per square meter in 1989-90 samples (Table 6.5). Also noticeable in the 1988-90 sampling period was the gradual disappearance of redbreast sunfish from the population. In comparison with the reference site at GCK 2.4, the fish density at MIK 0.78 was slightly higher (1.6 vs 1.2 fish per square meter). Both reference sites and MIK 0.78 suffered from low-water conditions as a result of a drought in 1987-88. Another factor that may have affected fish densities was a fish kill in Mitchell Branch in November 1988 that was traced to SD 170 (J. M. Loar, ESD, ORNL, March 17, 1989, personal communication). Although the kill occurred downstream of the section of MIK 0.78 where fish were absent, it probably adversely affected immigration into the site from the lowermost reaches of Mitchell Branch downstream of the toxic zone.

\subsubsection{Population biomass}

Fish biomass values (in grams wet weight per square meter) paralleled the 
Table 6.5. Fish densities (per square meter) in Mitchell Branch and Grassy Creek, a reference stream, January 1988-April/May 1990

\begin{tabular}{lcccccc}
\hline & MIK & MIK & MIK & MIK & MIK & GCK \\
Species` & 0.45 & 0.54 & 0.71 & 0.78 & $1.43^{b}$ & 2.4 \\
\hline
\end{tabular}

January 1988

Blacknose dace

Creek chub

Redbreast sunfish

White sucker

Total

$\mathrm{NF}^{\mathrm{r}}$

NF

Total

Creek chub

White sucker

Blacknose dace

Creek chub

Total

NF

NF

NF

0.2

0.5

0.9

0.1

March 1988

NF

NF

$\overline{1.1}$

NF

$<\frac{0.1}{0.6}$

July 1988
0.2

$\frac{0.3}{0.5}$
0.4

0.1

$\frac{<0.1}{0.5}$
Blacknose dace

Creek chub

Redbreast sunfish

Total

NF

NF

April/May 1989

October/November 1988

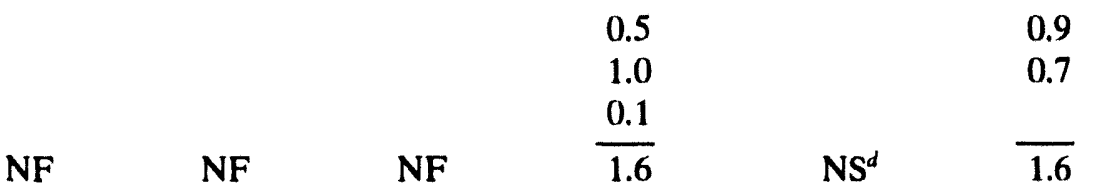

Blacknose dace

0.7

0.1

Creek chub

1.3

0.1

Striped shiner

White sucker

NF

NF

NF

$\overline{2.0}$

$<0.1$

$<0.1$

Total

NF

NF

NF

NS

0.2 
Table 6.5 (continued)

\begin{tabular}{lcccccc}
\hline & MIK & MIK & MIK & MIK & MIK & GCK \\
Species & 0.45 & 0.54 & 0.71 & 0.78 & $1.43^{b}$ & 2.4 \\
\hline
\end{tabular}

October 1989

Blacknose dace

Creek chub

Striped shiner

Central stoneroller

White sucker

Banded sculpin

Green sunfish

Total

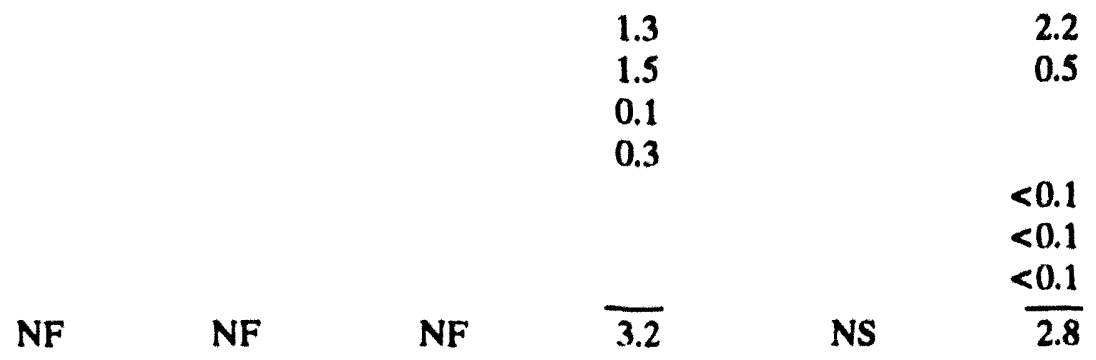

April/May 1990

Blacknose dace

Creek chub

Central stoneroller

Striped shiner

White sucker

Banded sculpin

Total

NF

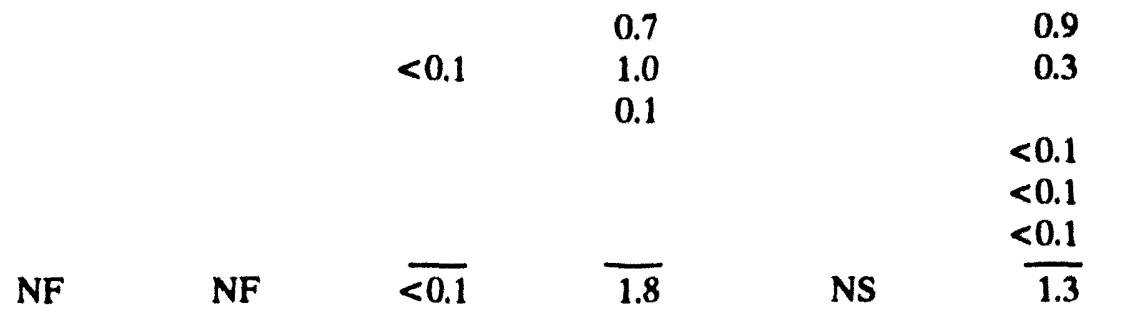

Species are blacknose dace, Rhinichthys atratulus; creek chub, Semotilus atromaculatus; striped shiner Luxilus chrysocephalus; central stoneroller, Campostoma anomalum; redbreast sunfish, Lepomis auritus; green sunfish, L. cyanellus; banded sculpin Cottus carolinae; and white sucker, Catostomus commersoni.

'Reference site on upper Mitchell Branch.

'NF = no fish taken in sample.

NS $=$ not sampled.

Note: MIK = Mitchell Branch kilometer; GCK = Grassy Creek kilometer.

trends observed in population density

(Table 6.6). Biomass at MIK 0.78 declined about $50 \%$ between the first year's surveys (mean of 10.6) and the surveys of 1988-90 (mean of 5.6), further indicating that adverse impacts affected fish populations in Mitchell Branch. When compared with the reference site on Grassy Creek (GCK 2.4), biomass at MIK 0.78 was high.

\subsubsection{Annual production}

Annual production ( $\mathrm{g}$ wet $\mathrm{wt} \cdot \mathrm{m}^{-2}$. year $\left.{ }^{-1}\right)$ of individual species and the fish community was calculated for MIK 0.78 and GCK 2.4 from spring to spring for 1987-90. In general, production increased each year for both individual species and the whole community (Table 6.7). The 
Table 6.6. Fish biomese (in grams per square moter) in Mitchell Branch and Orasy Creek, a reference stream, from January 1988-AprilMay 1990

\begin{tabular}{lcccccc}
\hline & MIK & MIK & MIK & MIK & MIK & OCK \\
Species" & 0.45 & 0.54 & 0.71 & 0.78 & $1.43^{b}$ & 2.4 \\
\hline
\end{tabular}

January 1988

Blacknose dace

Creek chub

Blacknose dace

Creek chub

Redbreast sunfish

White sucker

Total

NF

NF

NF

NF

NF

NF

March 1988

Blacknose dace

Creek chub

Whitc sucker

Total

Creek chub

Total

NF

NF

NF

0.2

4.1

$\overline{4.3}$

July 1988

0.4

1.8

0.4

5.0

0.5

$<0$.

0.4

0.1

0.4

0.5

$\overline{5.9} \quad \overline{0.1}$

$\frac{0.2}{1.1}$

$<0.1$

$\frac{0.2}{0.2}$

NF

0.3

0.2

0.6

NF

1.1

October/November 1988

Blacknose dace

Creek chub

Redbreast sunfish

Total

NF

NF

NF

Blacknose dace

Creek chub

Striped shiner

White sucker

Total

NF

NF

NF

1.4

0.2

7.2

0.4

0.1

0.4

0.9

2.1

1.9

$\frac{3.4}{5.9}$

NS

$\overline{2.8}$

April/May 1989 
Table 6.6 (continued)

\begin{tabular}{ccccccc}
\hline & MIK & MIK & MIK & MIK & MIK & OCK \\
Species" & 0.45 & 0.54 & 0.71 & 0.78 & $1.43^{b}$ & 2.4 \\
\hline
\end{tabular}

October 1989

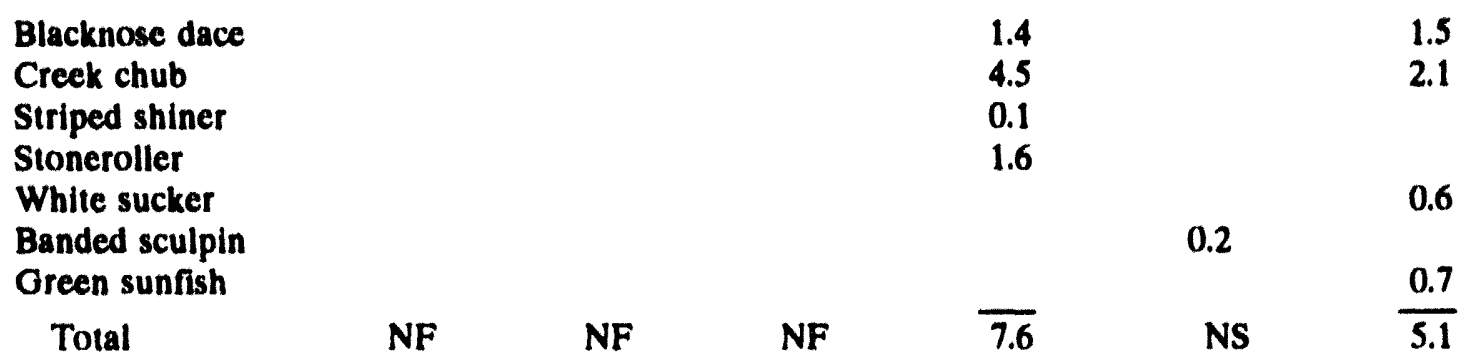

April/May 1990

Blacknose dace

Creek chub

Stoneroller

Striped shiner

White sucker

Banded sculpin

Total

NF

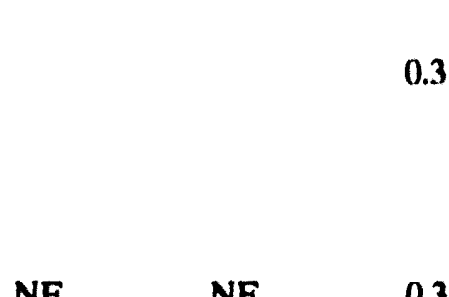

Species are blacknose dace, Rhinichthys atratulus; creek chub, Semotilus atromaculatus; striped shiner Notropis chrysocephalus; stoneroller, Campostoma anomalum; redbreast sunfish, Lepomis auritus; green sunfish, L. cyanellus; banded sculpin Cottus carolinae; and white sucker, Catostomus commersoni.

'Reference site on upper Mitchell Branch.

$\mathrm{NF}=$ no fish taken in sample.

NS $=$ not sampled.

Note: MIK = Mitchell Branch kilometer; GCK $=$ Grassy Creek kilometer.

exceptions were a decline in production of redbreast sunfish at MIK 0.78 after $1987-88$ and a dip in production for creek chub and blacknose dace at GCK 2.40 in 1988--89. The pattern of increasing production may be part of a general recovery from drought conditions in 1987 and 1988.

Total production at MIK 0.78 was 7 to 17 times higher than at GCK 2.4. The higher productivity of the disturbed Mitchell Branch site may reflect increased primary production resulting from nutrient enrichment and/or canopy removal. Elwood et al. (1981) demonstrated increased primary production following phosphorus enrichment of an nonpolluted stream. Such an enrichment may be occurring in Mitchell Branch as a result of plant discharges. Minshall (1978) presented some data for nonpolluted streams showing that mean annual gross primary production was greater in open 
Table 6.7. Annual production (in grams wet weight, per square meter per year \pm 1 SE) and production to blomess (P/B) ratios, in parenthesis, in Mitchell Branch and Grassy

Croek from spring 1987 to spring 1990

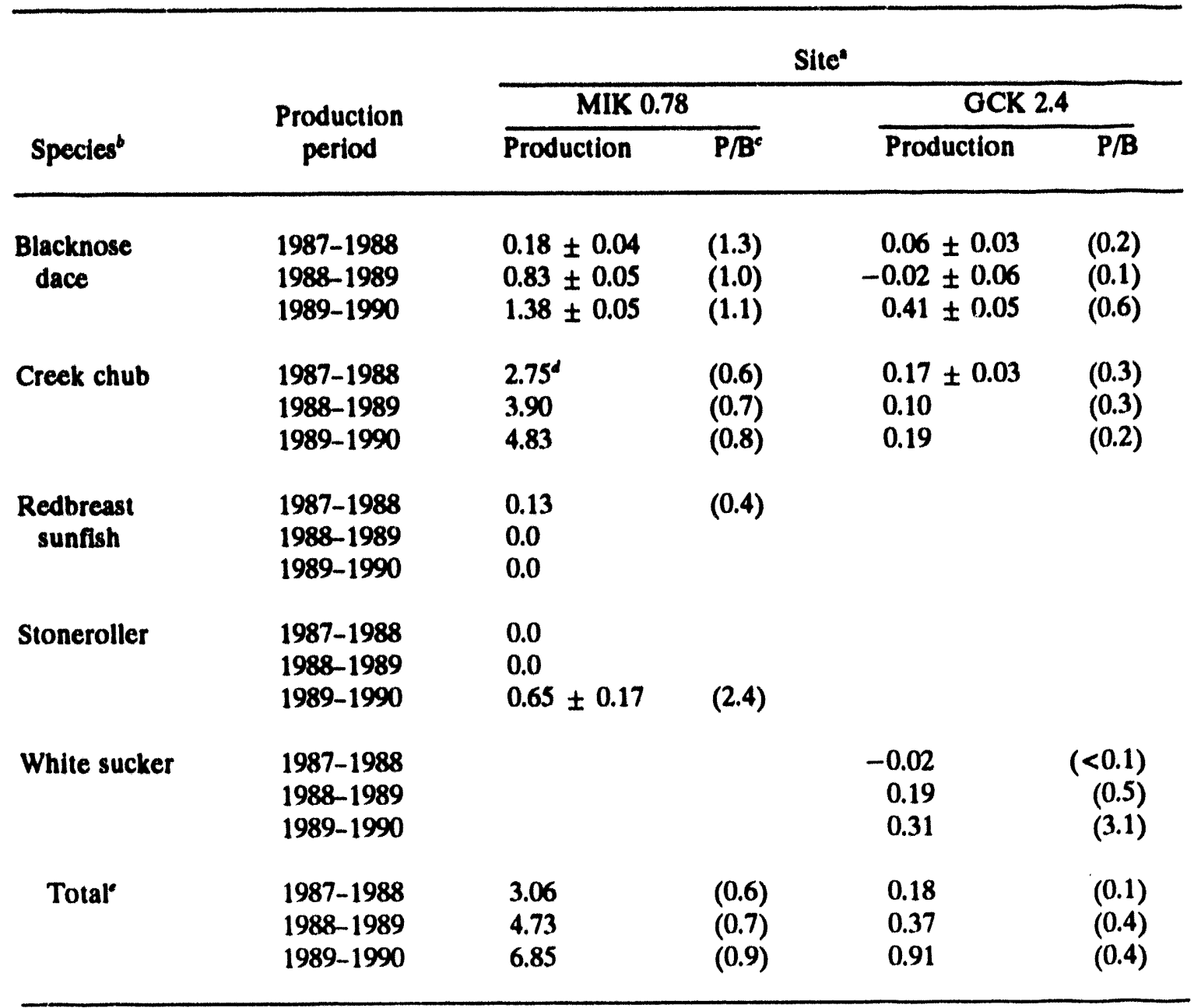

MIK = Mitchell Branch kilometer; GCK = Grassy Creek kilometer.

'Species are blacknose dace, Rhinichthys atratulus; creek chub, Semotilus atromaculatus; striped shiner Luxilus chrysocephalus; central stoneroller Campostoma anomalum; redbreast sunfish, Lepomis auritus; and white sucker, Catostomus commersoni.

'Biomass (B) is a mean of beginning and ending spring values for that production period.

standard error could not be calculated because some size classes have only one fish per class.

Totals include miscellaneous species (green sunfish L. cyanellus, and banded sculpin, Cottus carolinae) not listed.

streams than in streams with a closed canopy. The Grassy Creek canopy is more closed than that of Mitchell Branch. Higher primary production should sustain higher levels of secondary production, with greater numbers of benthic invertebrates available for consumption by fish species. However, secondary production of benthic invertebrates in 1986 sampling of MIK 0.78 was generally lower than other published 
values (Smith 1993). Secondary production estimates for MIK 0.78 were also lower than production estimates for unaffected headwater streams in the Oak Ridge area (Smith 1993). Data to determine whether this trend in benthic production continues for 1988-90 are not yet available.

New fish species found at MIK 0.78 in 1989-90 demonstrate that immigration (also see Sect. 5.3.3) provides an added component of the production value. Although new species were also added to the fauna at GCK 2.4, their occurrence was not as sustained as that at MIK 0.78 .

The annual fish production values for MIK 0.78 are in the middle of the range of other published production in warmwater streams (Table 6.8). The MIK 0.78 production was generally higher than that reported by Neves and Pardue (1983) for second-order streams in Virginia but lower than production in second-order streams in Kentucky (Lotrich 1973, Small 1975). The same trend held for comparisons of species. The production rate of blacknose dace was very similar between MIK $0.78\left(0.18\right.$ to $1.38 \mathrm{~g}$ wet $\mathrm{wt} \cdot \mathrm{m}^{-2} \cdot$ year $\left.^{-1}\right)$ and Virginia streams $(0.30$ to $0.38 \mathrm{~g}$ wet $\mathrm{wt} \cdot \mathrm{m}^{-2} \cdot$ year $\left.^{-1}\right)$. For creek chub, the production rate at MIK 0.78 (2.75 to $4.83 \mathrm{~g}$ wet $\left.\mathrm{wt} \cdot \mathrm{m}^{-2} \cdot \mathrm{year}^{-1}\right)$ was lower than in Kentucky streams $\left(7.28 \mathrm{~g}\right.$ wet $\mathrm{wt} \cdot \mathrm{m}^{-2} \cdot$ year $^{-1}$, Lotrich 1973). The production to biomass (P/B) ratio at MIK 0.78 was 0.6 to 0.9 ;

Table 6.8. Annual production of fish communities in warm water streams in the Southeast

\begin{tabular}{lccl}
\hline \multicolumn{1}{c}{ Stream (state) } & $\begin{array}{c}\text { Stream } \\
\text { order }\end{array}$ & $\begin{array}{c}\text { Production } \\
\left(\mathrm{g} \cdot \mathrm{m}^{-2} \cdot \mathrm{year}^{-1}\right. \\
\text { wet wt) }\end{array}$ & Reference $^{a}$ \\
\hline J. Carpenter Branch (Ky.) & 1st & 8.55 & Lotrich 1973 \\
Clemmons Creek (Ky.) & 2nd & 10.55 & Lotrich 1973 \\
Steeles Run (Ky.) & 2nd & $12.0^{b}$ & Small 1975 \\
& 2nd & $15.8^{b}$ & \\
Guys Run (Va.) & 2nd & 2.84 & Neves and \\
& $\begin{array}{l}\text { 2nd } \\
\text { 2nd }\end{array}$ & 3.16 & Pardue 1983 \\
Little Cooter Prairie (Ga.) & $c$ & 17.07 & Freeman and \\
& & & Freeman 1985 \\
\hline
\end{tabular}

aComplete reference citations may be found in Sect. 7 of this document (J. G. Smith et al., 1993, Second Report on the Oak Ridge K-25 Site Biological Monitoring and Abatement Program for Mitchell Branch, ORNL/TM-12150, Oak Ridge National Laboratory, Oak Ridge, Tenn.).

"Values converted from dry wt by using the conversion factor in T. F. Waters 1977, "Secondary production in inland waters," Adv. Ecol. Res. 10:91-164.

'Blackwater swamp; part of the Okefenokee Swamp. 
these values were much lower than the other published value, 3.2, for a blackwater swamp in Georgia (Freeman and Freeman 1985).

\subsubsection{Condition factors}

Comparisons of condition factors $(K)$ should provide information on the relative well-being of the fish because those with more weight per length have a higher condition factor (Everhart et al. 1975). Data were available for a statistical comparison of condition factors for blacknose dace and creek chub between MIK 0.78 and GCK 2.4 during January 1988, March 1988, October/November 1988, April/May 1989, October 1989, and April/May 1990.
For the blacknose dace and the creek chub, condition factors were significantly greater at MIK 0.78 than at GCK 2.4 in four of six comparisons (Table 6.9). Both species demonstrated significant differences at the same sampling periods. However, a consistent trend for improvement or decline was not apparent over the 1988-90 period (also see Sect. 5.3.1.4). The higher condition factors of fish in Mitchell Branch vs Grassy Creek suggest that individual fish surviving stressful conditions in Mitchell Branch generally remained in good health. However, caution must be exercised in the interpretation of condition factors because they may be relatively insensitive to environmental conditions or nutritional status (Loar et al. 1985).

Table 6.9. An analysis of variance comparison of fish condition factors between Mitchell Branch and Grassy Creek, January 1988-April/May 1990

\begin{tabular}{lllll}
\hline & & \multicolumn{3}{c}{ Site $^{a}$} \\
\cline { 5 - 5 } Species & Sampling date & MIK 0.78 & GCK 2.4 & \multicolumn{1}{c}{$p^{b}$} \\
\hline \multirow{3}{*}{ Blacknose dace } & January 1988 & 1.00 & 0.97 & 0.40 \\
& March 1988 & $1.17^{c}$ & 0.83 & 0.0001 \\
& Oct/Nov 1988 & $0.95^{c}$ & 0.85 & 0.004 \\
& April/May 1989 & 1.07 & 1.06 & 0.90 \\
& October 1989 & $1.15^{c}$ & 0.89 & 0.0001 \\
Creek chub & April/May 1990 & $1.04^{c}$ & 0.89 & 0.0001 \\
& & & & \\
& January 1988 & 1.03 & 0.97 & 0.10 \\
& March 1988 & $1.17^{c}$ & 0.96 & 0.005 \\
& Oct/Nov 1988 & $0.99^{c}$ & 0.92 & 0.04 \\
& April/May 1989 & 1.18 & 1.10 & 0.07 \\
& October 1989 & $1.02^{c}$ & 0.91 & 0.0001 \\
& April/May 1990 & $1.16^{c}$ & 0.98 & 0.0001 \\
& & & & \\
\hline
\end{tabular}

${ }^{a} \mathrm{MIK}=$ Mitchell Branch kilometer; GCK $=$ Grassy Creek kilometer. ${ }^{b} P=$ probability level.

'MIK 0.78 values that are statistically significantly different from GCK 2.4 values. 
Seasonal comparisons of condition factors did not show a trend for blacknose dace but did indicate for the creek chub that the spring samples were statistically greater $(p<0.05)$ than the other sampling periods. These spring increases for creek chubs may reflect preparations for spawning.

\subsubsection{Conclusions}

During the 1988-90 sampling period, fish populations in Mitchell Branch revealed substantial, adverse impacts. Species richness, density, and biomass of fish in Mitchell Branch were all low, with the extent of impact related spatially to operations at the Oak Ridge K-25 Site. Additionally, a steady decline in the robustness of the fish community was observed. The absence of fish below SD 170, which enters Mitchell Branch at MIK 0.76 , strongly suggests that toxic effluents are entering the stream at this site. Toxicants may have also entered through discharges from the K-1407-E/F ponds, SD 180, and/or SD 190. Although residual chlorine is a toxicant in Mitchell Branch (see Sect. 3), other stressors such as siltation could also contribute to the observed effects on the fish populations.

\subsubsection{Future Studies}

Earlier plans for assessing the fish community in Mitchell Branch involved quarterly sampling of the fish populations for the first 2 years (Loar et al. 1991, Ryon 1993). Because fish populations in most of Mitchell Branch have disappeared, sampling was restricted to the spring and fall periods. Future sampling will continue on this frequency. Data indicate an adverse impact from MIK 0.78 downstream to MIK 0.45, but because fish are taken in an impounded area of lower Mitchell Branch, an attempt will be made to define the downstream edge of toxic conditions in lower Mitchell Branch.

Plans to assess impacts at the individual level, by examining fecundity or feeding patterns, will be implemented only if population densities and biomass stabilize. Because immigration of individuals and species into Mitchell Branch from Poplar Creek is occurring, further qualitative sampling will be conducted to assess immigration. This sampling may be a cooperative effort with sampling for the bioaccumulation task. 


\section{REFERENCES}

Adams, S. M. 1993. Biological indicators of contaminant related stress. IN J. M. Loar, (ed.), Fourth annual report on the ORNL Biological Monitoring and Abatement Program. ORNL/TM-11544. Oak Ridge National Laboratory, Oak Ridge, Tenn.

Adams, S. M., and R. B. McLean. 1985. Estimation of largemouth bass, Micropterus salmoides Lacepede, growth using the liver somatic index and physiological variables. J. Fish Biol. 26:111-126.

Addison, R. F. 1984. Hepatic mixed function oxidase (MFO) induction in fish as a possible biological monitoring system. pp. 51-60. IN V. W. Cairns, P. V. Hodson, and J. O. Nriagu (eds.), Contaminant Effects on Fisheries. John Wiley \& Sons, New York.

Ahokas, J. T., N. T. Kärki, A. Oikari, and A. Soivio. 1976. Mixed function monooxygenase of fish as an indicator of pollution of Aquatic Environment by industrial effluent. Bull. Environ. Contam. Toxicol. 16:270-274.

Allain, C. C., L. S. Poon, C. S. G. Chan, W. Richmond, and P. C. Fu. 1974. Enzymatic determination of total serum cholesterol. Clin. Chem. 20:470-475.

Ashwood, T. L., C. R. Olsen, I. L. Larsen, and P. D. Lowery. 1986. Sediment contamination in streams surrounding the Oak Ridge Gaseous Diffusion Plant. ORNL/TM-9791. Oak Ridge National Laboratory, Oak Ridge, Tenn.

Bagenal, T. B. 1978. Methods for assessment of fish production in fresh waters. Blackwell Scientific Publications. Oxford.
Bennett, G. W. 1970. Management of lakes and ponds. Van Nostra. Reinhold Company, New York.

Bergmeyer, H. U., P. Scheibe, and A. W. Wahlefeld. 1978. Optimization of methods for aspartate aminotransferase and alanine aminotransferase. Clin. Chem. 24:58-73.

Boyle, J. W., R. Blumberg, S. J. Cotter, G. S. Hill, C. R. Kerley, R. H. Ketelle, R. L. Kroodsma, D. W. Lee, R. C. Martin, R. D. Roop, D. N. Secora, W. P. Staub, and R. E. Thoma. 1982. Environmental analysis of the operation of the Oak Ridge National Laboratory (X-10 Site). ORNL-5870. Oak Ridge National Laboratory, Oak Ridge, Tenn.

Bradford, M. M. 1976. A rapid and sensitive method for the quantitation of protein utilizing the principle of protein-dye binding. Anal. Biochem. 72:248-254.

Brown, D. A., R. W. Gossett, G. P. Hershelman, C. F. Ward, A. M. Westcott, and J. N. Cross. 1986. Municipal wastewater contamination in the Southern California Bight: Part I-Metal and organic contaminants in sediments and organisms. Mar. Environ. Res. 18:291-310.

Bucolo, G., and H. David. 1973. Quantitative determination of serum triglycerides by the use of enzymes. Clin. Chem. 19:476-482.

Burke, M. D., and R. T. Mayer. 1974. Ethoxyresorufin: Direct fluorimetric assay of a microsomal O-dealkylation which is preferentially inducible by 3-methylcholanthrene. Drug Metah. Dispos. 2:583-588.

Carle, F. L., and M. R. Strub. 1978. A new method for estimating population 
size from removal data. Biometrics 34:621-630.

Davis, J. R. 1972. The spawning behavior, fecundity rates, and food habits of the redbreast sunfish in southeastern North Carolina. Proc. Annu. Conf. Southeast. Assoc. Game Fish Comm. 25:556-560.

DOE (U.S. Department of Energy). 1979. Environmental assessment of the Oak Ridge Gaseous Diffusion Plant site. DOE/EA-0106. Technical Information Center, U.S. Department of Energy, Oak Ridge, Tenn.

Doumas, B. T., W. A. Watson, and H. G. Biggs. 1972. Standard methods in clinical chemistry. Clin. Chem. Acta. 31:87.

Elliott, J. M. 1977. Some methods for the statistical analysis of samples of benthic invertebrates. Freshwater Biological Association, Ambleside, England. Sci. Pub. No. 25.

Elwood, J. W., J. D. Newbold, A. F. Trimble, and R. W. Stark. 1981. The limiting role of phosphorus in a woodland stream ecosystem: Effects of $P$ enrichment on leaf decomposition and primary producers. Ecology 62:146-156.

EPA (U.S. Environmental Protection Agency). 1980. Interim methods for the sampling and analysis of priority pollutants in sediments and fish tissues. EPA 600/4-81-055. U.S. Environmental Protection Agency, Environmental Monitoring and Support Laboratory, Cincinnati, Oh.

EPA (U.S. Environmental Protection Agency). 1983. Extraction and analysis of priority pollutants in biological tissue, Method PPD 12/83.

U.S. Environmental Protection Agency, Environmental Sciences Division, Region IV, Analytical Support Branch, Athens, Ga. Mimeo.
EPA (U.S. Environmental Protection Agency). 1986. Authorization to discharge under the National Pollutant Discharge Elimination System, Permit No. TNO002950, Oak Ridge Gaseous Diffusion Plant. U.S. Environmental Protection Agency, Region IV, Atlanta, Ga.

EPA (U.S. Environmental Protection Agency). 1990. Toxic substance spreadsheet. U.S. Environmental Protection Agency, Region IV, Water Management Division, Atlanta, Ga. Mimeo.

Everhart, W. H., A. W. Eipper, and D. W. Youngs. 1975. Principles of Fishery Science. Cornell University Press, Ithaca, N.Y.

Fausch, K. D., J. Lyons, J. R. Karr, and P. L. Angermeier. 1990. Fish communities as indicators of environmental degradation. Am. Fish. Soc. Symp. 8:123-144.

Fletcher, G. L, M. J. King, J. W. Kiceniuk, and R. F. Addison. 1982. Liver hypertrophy in winter flounder following exposure to experimentally oiled sediments. Comp. Biochem. Physiol. 73C:45762.

FDA (U.S. Department of Agriculture Food and Drug Administration). 1984a. Polychlorinated biphenyls (PCBs) in fish and shellfish: Reduction of tolerances, Final Decision. Fed. Regist. 49(100):21520.

FDA (U.S. Department of Agirculture Food and Drug Administration). 1984b. Action level for methylmercury in fish. Fed. Regist. 49(224):456-463.

Forlin, L., and T. Anderson. 1985. Storage conditions of rainbow trout liver cytochrome P-450 and conjugating enzymes. Comp. Biochem. Physiol. 3:569-572.

Freeman, B. J., and M. C. Freeman. 1985. Production of fishes in a subtropical blackwater ecosystem: The 
Okefenokee swamp. Limnol. Oceanogr. 30:686-692.

Garman, G. C., and T. F. Waters. 1983. Use of the size-frequency (Hynes) method to estimate annual production of a stream fish population. Can. J. Fish. Aquat. Sci. 40:2030-2034. Greeson, P. E., T. A. Ehlke, G. A. Irwin, B. W. Lium, and K. V. Slack. 1977. Methods for collection and analysis of aquatic biological and microbiological samples. Book 5, Chap. 4A, pp. 1-332. IN U.S. Geological Survey, Techniques of water-resources Investigations of the United States Geological Survey. U.S. Government Printing Office, Washington, D.C.

Heath, A. G. 1987. Water pollution and fish physiology. CRC Press, Boca Raton, Fla.

Heidinger, R. C., and S. D. Crawford. 1977. Effect of temperature and feeding rate on the liver-somatic index of the largemouth bass, Micropterus salmoides. J. Fish. Res. Bd. Can. 34:633-638.

Henry, R. J., D. C. Cannon, and J. W. Winkelman. 1974. Clinical chemistry; principles and technics. 2nd ed. Harper and Row, Hagerstown, Md.

Hile, R. 1936. Age and growth of the cisco, Leucichthys artedi (LeSeur), in the lakes of the northeastern highlands, Wisconsin. U.S. Bur. Fish. Bull. 48:211-317.

Hinton, D. E., and J. A. Couch. 1984. Pathological measures of marine pollution effects. pp. 7-32. IN H. H. Harris (ed.), Concepts in Marine Pollution Measurements. Maryland Sea Grant College, University of Maryland, College Park, Md.

Hinzman, R. L., S. M. Adams, M. C. Black, H. L. Boston, W. Burton, A. J. Gatz, Jr., M. S. Greeley, Jr., W. R. Hill, J. M. Loar,
J. F. McCarthy, M. J. Peterson, M. G. Ryon, J. G. Smith, G. R. Southworth, A. J. Stewart, and V. R. Tolbert. 1993. Second report on the Oak Ridge Y-12 Plant Biological Monitoring and Abatement Program for East Fork Poplar Creek. Y/TS-888. Oak Ridge National Laboratory, Oak Ridge, Tenn.

Hoffman, F. O., B. G. Blaylock, C. C. Travis, K. L. Daniels, E. L. Etnier, K. E. Cowser, and C. W. Weber. 1984. Preliminary screening of contaminants in sediments. ORNL/TM-9730. Oak Ridge National Laboratory, Oak Ridge, Tenn. Horning, W. B., and C. I. Weber 1985.

Short-term methods for estimating the chronic toxicity of effluents and receiving waters to freshwater organisms. EPA/600/4-85/014. U.S. Environmental Protection Agency, Cincinnati, Oh.

Horowitz, R. J. 1978. Temporal variability patterns and the distributional patterns of stream fishes. Ecol. Monog. 48:307-321.

Jearld, A. 1983. Age determination. pp. 301-324. IN L. A. Nielsen and D. L. Johnson (eds.), Fisheries Techniques. Southern Printing Co., Blacksburg, Va.

Johannesen, K. M., and J. W. DePierre. 1978. Measurements of cytochrome $P_{450}$ in the presence of large amounts of contaminating hemoglobin and methemoglobin. Anal. Biochem. 86:725-32.

Karr, J. R. 1981. Assessment of biotic integrity using fish communities. Fisheries 6:21-27.

Karr, J. R. 1987. Biological monitoring and assessment: A conceptual framework. Environ. Manag. 11:249-256.

Karr, J. R. 1991. Biological integrity: A long-neglected aspect of water resource management. Ecol. Appl. 1:165-184. 
Karr, J. R., K. D. Fausch, P. L. Angermeier, P. R. Yant, and I. J. Schlosser. 1986. Assessing biological integrity in running waters: A method and its rationale. Illinois Natural History Survey Special Publication 5.

Kasten, J. L. 1986. Resource Management Plan for the Oak Ridge Reservation, Vol. 21: Water Conservation Plan for the Oak Ridge Reservation. ORNL/ESH-1/N21. Oak Ridge National Laboratory, Oak Ridge, Tenn.

Kszos, L. A., A. J. Stewart, L. F. Wicker, L. E. Roberson, and T. L. Phipps. 1988. Environmental Sciences Division Toxicology Laboratory Quality Assurance Program. QAP-X-89-ES-002. Oak Ridge National Laboratory, Oak Ridge, Tenn.

Kszos, L. A., A. J. Stewart, and P. A. Taylor. 1992. An evaluation of nickel toxicity to Ceriodaphnia dubia and Daphnia magna in a contaminated stream and in laboratory tests. Environ. Toxicol. Chem 11:1001-1012.

Kszos, L. A., A. J. Stewart, L. F. Wicker, and G. M. Logsdon. 1988. Environmental Sciences Division Toxicology Laboratory Standard Operating Procedures. ORNL/TM-11194. Oak Ridge National Laboratory, Oak Ridge, Tenn.

Loar, J. M., F. A. Burkhart, G. F. Cada, J. W. Huckabee, J. T. Kitchings, K. D. Kumar, A. M. Sasson, J. A. Solomon, and J. D. Story. 1981. Ecological studies in the vicinity of the Oak Ridge Gaseous Diffusion Plant. ORNL/TM-6714. Oak Ridge National Laboratory, Oak Ridge, Tenn.

Loar, J. M., M. J. Sale, G. F. Cada, D. K. Cox, R. M. Cushman, G. K. Eddlemon, J. L. Elmore,
A. J. Gatz, Jr., P. Kanciruk, J. A. Solomon, and D. S. Vaughn. 1985. Application of habitat evaluation models in southern Appalachian trout streams. ORNL/TM-9323. Oak Ridge National Laboratory, Oak Ridge, Tenn.

Loar, J. M., J. M. Giddings, S. M. Adams, J. F. McCarthy, and G. R. Southworth. 1989. The Oak Ridge Y-12 Plant Biological Monitoring and Abatement Program for East Fork Poplar Creek. TM-10265. Oak Ridge National Laboratory, Oak Ridge, Tennessee.

Loar, J. M., S. M. Adams, B. G. Blaylock, H. L. Boston, M. A. Huston, B. L. Kimmel, C. R. Olsen, J. G. Smith, G. R. Southworth, A. J. Stewart, and B. T. Walton. 1991a. Oak Ridge National Laboratory Biological Monitoring and Abatement Program for White Oak Creek watershed and the Clinch River. ORNL/TM-10370. Oak Ridge National Laboratory, Oak Ridge, Tenn.

Loar, J. M., L. M. Adams, S. M. Adams, M. G. Ryon, J. G. Smith, G. R. Southworth, and A. J. Stewart. 1991b. Oak Ridge Gaseous Diffusion Plant Biological Monitoring and Abatement Program for Mitchell Branch. ORNL/TM-11965. Oak Ridge National Laboratory, Oak Ridge, Tenn.

Loar, J. M., S. M. Adams, L. J. Allison, M. C. Black, H. L. Boston, A. J. Gatz, Jr., R. L. Hinzman, M. A. Huston, B. D. Jimenez, J. F. McCarthy, S. D. Reagan, J. G. Smith, G. R. Southworth, A. J. Stewart. 1992. First report on the Oak Ridge Y-12 Plant Biological Monitoring and Abatement Program for East Fork Poplar Creek. Y/TS-886. Oak Ridge Y-12 Plant, Oak Ridge, Tenn.

Loar, J. M., S. M. Adams, R. D. Bailey, B. G. Blaylock, H. L. Boston, 
W. R. Hill, M. A. Huston,

B. D. Jimenez, L. Meyers-Schöne,

D. A. Mohrbacher, M. J. Peterson,

M. G. Ryon, K. L. Shepard,

L. R. Shugart, J. G. Smith,

G. R. Southworth, A. J. Stewart,

C. K. Valentine, B. T. Walton, and

A. E. Waters. 1993. Third report on

the Oak Ridge National Laboratory

Biological Monitoring and Abatement

Program for White Oak Creek

watershed and the Clinch River.

ORNL/TM-11358. Oak Ridge

National Laboratory, Oak Ridge,

Tenn.

Lotrich, V. A. 1973. Growth, production, and community composition of fishes inhabiting a first-, second-, and thirdorder stream of eastern Kentucky.

Ecol. Monogr. 43:377-397.

Lowe, T. P., T. W. May,

W. G. Brumbaugh, and D. A. Kane.

1985. National contaminant

biomonitoring program: Concen-

trations of seven elements in

freshwater fish, 1978-1981. Arch.

Environ. Contam. Toxicol. 14:373-388.

Lowery, J. F., P. H. Counts,

H. L. Edmiston, and F. D. Edwards.

1987. Water resources data for

Tennessee, Water Year 1986.

Report No. USGS/WRD/HD-87/225.

U.S. Geological Survey, Nashville, Tenn.

Martin Marietta Energy Systems, Inc. 1983. Environmental and effluent analysis manual. Martin Marietta Energy Systems, Inc., Oak Ridge, Tenn.

McElravy, E. P., G. A. Lamberti, and V. H. Resh. 1989. Year-to-year variation in the aquatic macroinvertebrate fauna of a northern California stream. J. N. Am. Benthol. Soc. 8:51-63.

McGaha, M. A. 1989a. Toxicity monitoring at ORGDP July-September 1988.
K/QT-288. Oak Ridge Gaseous Diffusion Plant, Oak Ridge, Tenn.

McGaha, M. A. 1989b. Toxicity monitoring at ORGDP October-December 1988. K/QT-0311. Oak Ridge Gaseous Diffusion Plant, Oak Ridge, Tenn.

McGaha, M. A. 1989c. Toxicity monitoring at ORGDP January-March 1989. K/QT-312. Oak Ridge Gaseous Diffusion Plant, Oak Ridge, Tenn.

McGaha, M. A. 1989d. Toxicity monitoring at ORGDP April-June 1989. K/QT-313. Oak Ridge Gaseous Diffusion Plant, Oak Ridge, Tenn.

McGaha, M. A. 1989e. Toxicity monitoring at ORGDP July-September 1988. K/QT-342. Oak Ridge Gaseous Diffusion Plant, Oak Ridge, Tenn.

McKee, M. J., A. C. Hendricks, and R. E. Ebel. 1983. Effects of Naphthalene on Benzo[a]pyrene hydroxylase and cytochrome P-450 in Fundulus heteroclitus. Aquat. Toxicol. 3:103-114.

McMaster, W. M. 1967. Hydrologic data for the Oak Ridge area, Tennessee. U.S. Geological Survey-Water Supply Paper No. 1838-N. U.S. Government Printing Office, Washington, D.C.

Minshall, G. W. 1978. Autotrophy in stream ecosystems. Bioscience 28:767-771.

NCCLS (National Committee for Clinical Laboratory Standards). 1979. NCCLS Approved Standards: ACS-1 Specification for Standard Protein Solution (Bovine Serum Albumin), 2nd ed. National Committee for Clinical Laboratory Standards, Villanova, $\mathrm{Pa}$.

NOAA (National Oceanic and Atmospheric Administration). 1990. Local climatological data: 1989 annual summary with comparative data, Oak Ridge, Tennessee. National Climatic Data Center, Asheville, N.C. 
Neff, J. M. 1978. Polycyclic Aromatic Hydrocarbons. Applied Science Publisher, Ltd., London.

Payne J. F., and W. R. Penrose. 1975. Induction of aryl hydrocarbon benzo $[a]$ pyrene hydroxylase in fish by petroleum. Bull. Environ. Contam. Toxicol. 14:112-116.

Pielou, E. C. 1977. Mathematical ecology. John Wiley and Sons, New York.

Platts, W. S., W. F. Megahan, and G. W. Minshall. 1983. Methods for evaluating stream, riparian, and biotic conditions. U.S. Forest Service General Technical Report INT-138. Intermountain Forest and Range Experimental Station, Ogden, Utah.

Railsback, S. F., B. D. Holcomb, and M. G. Ryon. 1989. A computer program for estimating fish population sizes and annual production rates. ORNL/TM-11061. Oak Ridge National Laboratory, Oak Ridge, Tenn.

Ricker, W. E. 1975. Computation and interpretation of biological statistics of fish populations. Bull. Fish. Res. Board Can. Ottawa.

Roche Diagnostic Systems. 1986. Reagent for total protein. Roche Diagnostic Systems Information Package, Item 44903 \& 44313. Nutley, N.J.

Ryon, M. G., and J. M. Loar. 1988. A checklist of fishes on the Department of Energy Oak Ridge Reservation. J. Tenn. Acad. Sci. 53:97-102.

Ryon, M. G. 1993. Fishes. IN J. G. Smith et al., First Report on the Oak Ridge K-25 Site Biological Monitoring and Abatement Program for Mitchell Branch. ORNL/TM-11073. Oak Ridge National Laboratory, Oak Ridge, Tenn.

SAS Institute, Inc. 1985a. SAS User's Guide: Basics, Version 5 Edition. SAS Institute, Inc., Cary, N.C.
SAS Institute, Inc. 1985b. SAS User's Guide: Statistics, Version 5 Edition. SAS Institute, Inc., Cary, N.C.

Scheib, W. J. 1987. ORGDP storm drain characterization. Final Report, K/HS-128 Part 2. Oak Ridge Gaseous Diffusion Plant, Oak Ridge, Tenn.

Shoemaker, J. L., L. A. Kszos, M. A. McGaha, P. E. Osborne, and R. Woods. 1990 . Toxicity monitoring at ORGDP October-December 1989. K/QT-373. Oak Ridge Gaseous Diffusion Plant, Oak Ridge, Tenn.

Small, J. W., Jr. 1975. Energy dynamics of benthic fishes in a small Kentucky stream. Ecology 56:827-840.

Smith, J. G. 1992a. Benthic macroinvertebrates. IN J. M. Loar (ed.), First report on the Oak Ridge Y-12 Plant Biological Monitoring and Abatement Program for East Fork Poplar Creek. Y/TS-886. Oak Ridge National Laboratory, Oak Ridge, Tenn.

Smith, J. G. 1992b. Benthic macroinvertebrates. IN J. M. Loar (ed.), Second report on the Oak Ridge National Laboratory Biological Monitoring and Abatement Program for White Oak Creek watershed and the Clinch River. ORNL/TM-10804. Oak Ridge National Laboratory, Oak Ridge, Tenn.

Smith, J. G. 1994. Benthic macroinvertebrates. pp. 6-1 to 6-20. IN J. M. Loar (ed.), Third report on the Oak Ridge National Laboratory Biological Monitoring and Abatement Program for White Oak Creek watershed and the Clinch River. ORNL/TM-11358. Oak Ridge National Laboratory, Oak Ridge, Tenn.

Smith, J. G., S. M. Adams, L. A. Kszos, J. M. Loar, M. G. Ryon, and G. R. Southworth. 1993. First Report on the Oak Ridge K-25 Site Biological Monitoring and Abatement Program for Mitchell Branch. 
ORNL/TM-11073. Oak Ridge National Laboratory, Oak Ridge, Tenn.

Sokal, R. R., and F. J. Rohlf. 1981. Biometry, 2nd ed. W. H. Freeman and Company, San Francisco, Calif.

Steel, R. G. D., and J. H. Torrie. 1960. Principles and procedures of statistics with special reference to the biological sciences. McGraw-Hill, Inc., New York.

Stegeman J. J., R. L. Binder, and A. Orren. 1979. Hepatic and extrahepatic microsomal electron transport components and mixed function oxygenases in the marine fish Stenotomus versicolor. Biochem. Pharmacol. 28:3421-3439.

TVA (Tennessee Valley Authority). 1985. Instream contaminant study, Task 4: Fish sampling and analysis. Report to U.S. Department of Energy, Oak Ridge Operations Office. Tennessee Valley Authority, Office of Natural Resources and Economic Development, Knoxville, Tenn.

TVA (Tennessee Valley Authority). 1986. Instream contaminant study, Task 5: Summary report. Report to U.S. Department of Energy, Oak Ridge Operations Office. Tennessee Valley Authority, Office of Natural Resources and Economic Development, Knoxville, Tenn.

Tietz, N. W. 1986. Textbook of Clinical Chemistry. W. B. Saunders, Co., Philadelphia, $\mathrm{Pa}$.
Tiffany, T. O., J. M. Jansen, C. A. Burtis, J. B. Overton, and C. D. Scott. 1972. Enzymatic kinetic rate and end-point analyses of substrate by use of a GEMSAEC fast analyzer. Clin. Chem. 18:829-840.

Travis, C. C., F. O. Hoffman, B. G. Blaylock, K. L. Daniel, C. S. Gist, C. W. Weber. 1986. Preliminary review of TVA fish sampling and analysis report. Report of Task Group Five to Oak Ridge Task Force. January 1986. Mimeo.

Weber, C. I. (ed.). 1973. Biological field and laboratory methods for measuring the quality of surface waters and effluents. EPA 670/4-73-001. National Environmental Research Center, U.S. Environmental Protection Agency, Cincinnati, Oh.

Whitley, L. S. 1982. Aquatic Oligochaeta. IN A. R. Brigham, W. U. Brigham, and A. Gnilka (eds.), Aquatic Insects and Oligochaetes of North and South Carolina. Midwest Aquatic Enterprises, Mahomet, Ill.

Wiederholm, T. 1984. Responses of aquatic insects to environmental pollution. pp. 508-557. IN V. H. Resh and D. M. Rosenberg (eds.), The Ecology of Aquatic Insects. Praeger Publishers, New York.

Zar, 1984. Biostatistical analysis.

Prentice-Hall, Inc., Englewood Cliffs, N.J. 
Appendix A

WATER QUALITY DATA FOR MITCHELL BRANCH 


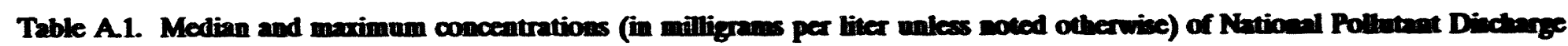
Elimination System paramelers in the water from lower Mitchell Branch

\begin{tabular}{|c|c|c|c|c|c|c|c|c|}
\hline \multirow[b]{3}{*}{ Parameter } & \multicolumn{8}{|c|}{ K-1700 } \\
\hline & \multicolumn{2}{|c|}{1986} & \multicolumn{2}{|c|}{1987} & \multicolumn{2}{|c|}{1988} & \multicolumn{2}{|c|}{1989} \\
\hline & Median & $\operatorname{Max}$ & Median & $\operatorname{Max}$ & Median & $\operatorname{Max}$ & Median & $\operatorname{Max}$ \\
\hline Aluminum, total & 0.1900 & 28000 & 0.1300 & 120000 & 0.1800 & 8.1000 & 0.2500 & 9.4000 \\
\hline Arsenic, total & $\mathbf{N A} \mathbf{A}^{\mathbf{b}}$ & NA & NA & NA & NA & NA & $<0.0050$ & 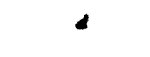 \\
\hline Barium, total & $<0.1$ & 2 & NA & NA & NA & NA & $<0.1000$ & 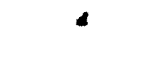 \\
\hline Beryllium, total & $<0.0010$ & $<0.0010$ & $<0.0010$ & $<0.0010$ & $<0.0010$ & $<0.0010$ & $<0.0010$ & $<0.0010$ \\
\hline Beta, total pCir & NA & NA & NA & NA & NA & NA & 18.5000 & 36.0000 \\
\hline Boron, total & 0.0410 & $\triangleleft$ & NA & $\mathbf{N A}$ & NA & NA & 0.0720 & 4 \\
\hline Cadmium & $<0.0020$ & 0.0020 & 0.0020 & 0.0120 & $<0.0020$ & 0.0026 & $<0.0020$ & 0.0030 \\
\hline Cesium & 100.0000 & $\bullet$ & 100.0000 & 100.0000 & 0.0000 & 100.0000 & 0.0000 & 0.0000 \\
\hline Chemical axygen demand & .7 .0000 & 7.0000 & 7.5000 & 38.0000 & 7.7500 & 688.0500 & $<5.0000$ & 159.0000 \\
\hline Chromium & 0.0100 & 0.0180 & 0.0100 & 0.0230 & $<0.0100$ & 0.0180 & $<0.0100$ & 0.0250 \\
\hline Cobalt, total & NA & NA & NA & NA & NA & NA & $<0.1000$ & $<$ \\
\hline Copper & NA & NA & NA & NA & NA & NA & 0.0110 & 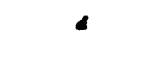 \\
\hline Flow $(L / s)$ & NA & NA & NA & NA & 23.1000 & 43.6000 & 25.1000 & 585000 \\
\hline Fluoride & 0.2000 & 1.0000 & 0.4000 & 6.0000 & 0.7000 & 14.0000 & 0.3000 & 1.7000 \\
\hline Iron, total & NA & NA & NA & NA & NA & NA & 202000 & 32000 \\
\hline Lead & 0.0040 & 0.0070 & 0.0040 & 0.0360 & $<0.0040$ & 0.0230 & $<0.0040$ & 0.0130 \\
\hline Magnesium, total & NA & NA & NA & NA & $\mathbf{N A}$ & NA & 5.9000 & 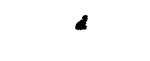 \\
\hline Manganese, total & NA & NA & NA & NA & NA & NA & 0.0930 & 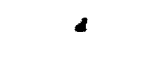 \\
\hline Mercury, total & $<0.0002$ & 0.0002 & 0.0002 & 0.0010 & $<0.0002$ & 0.0007 & $<0.0002$ & 0.00065 \\
\hline Molybdenum, total & NA & NA & NA & $\mathbf{N A}$ & NA & NA & $<0.0100$ & $<$ \\
\hline $\begin{array}{l}\text { Neptunium } \\
\text { Nicikel }\end{array}$ & $<1.0000$ & : & $<1.0000$ & $<\begin{array}{l}<.0000 \\
\text { NA }\end{array}$ & $\begin{array}{l}0.1200 \\
\text { NA }\end{array}$ & $\begin{array}{l}1.0000 \\
\text { NA }\end{array}$ & $\begin{array}{r}0.0950 \\
<0.0500\end{array}$ & 0.1600 \\
\hline $\begin{array}{l}\text { Nicikel } \\
\text { Nitrate as N }\end{array}$ & $\begin{array}{l}\text { NA } \\
0.4400\end{array}$ & $\begin{array}{l}\text { NA } \\
5.7200\end{array}$ & $\begin{array}{l}\text { NA } \\
0.4500\end{array}$ & $\begin{array}{l}\text { NA } \\
7.6800\end{array}$ & $\begin{array}{l}\text { NA } \\
0.3300\end{array}$ & $\begin{array}{l}\text { NA } \\
3.7000\end{array}$ & 0.4000 & 4.0000 \\
\hline Oil and grease & 20000 & 4.0000 & $<20000$ & 2.0000 & $<20000$ & 5.5000 & $<20000$ & $<20000$ \\
\hline
\end{tabular}


Table A.1 (contioned)

\begin{tabular}{|c|c|c|c|c|c|c|c|c|}
\hline \multirow[b]{3}{*}{ Parameter } & \multicolumn{8}{|c|}{$\mathrm{K}-1700^{\circ}$} \\
\hline & \multicolumn{2}{|c|}{1986} & \multicolumn{2}{|c|}{1987} & \multicolumn{2}{|c|}{1988} & \multicolumn{2}{|c|}{1989} \\
\hline & Median & $\operatorname{Max}$ & Median & $\overline{\operatorname{Max}}$ & Median & $\operatorname{Max}$ & Median & Max \\
\hline $\mathrm{pH}$ & NA & NA & NA & NA & 7.7000 & 8.8000 & 7.6000 & 8.6000 \\
\hline Phenols, total & $\mathbf{N A}$ & NA & 3.0000 & $\cdot$ & NA & NA & $\mathbf{N A}$ & $\mathbf{N A}$ \\
\hline Phosphorus, total & NA & NA & NA & NA & NA & $\mathbf{N A}$ & $<0.2000$ & 2 \\
\hline Plutonium & 1.0000 & b & 1.0000 & 20000 & -0.04 & 37.0000 & 0.0750 & 0.5900 \\
\hline Selenium, total & $<0.0050$ & $<0.0050$ & $<\cup .0050$ & $<0.0050$ & $<0.0050$ & 0.0059 & $<0.0050$ & 0.0050 \\
\hline Sitver & $<0.0100$ & $<0.0100$ & $<0.0100$ & $<0.0100$ & $<0.0100$ & 0.0240 & $<0.0100$ & 0.0100 \\
\hline Technetium (pCi/L) & 110.0000 & 110.0000 & 149.0000 & 229.0000 & 140.0000 & 295.0000 & 63.0000 & 157.0000 \\
\hline Temperature & NA & NA & NA & $\mathbf{N A}$ & 16.5500 & 320000 & 155000 & 30.3000 \\
\hline Titanium, total & $<0.0030$ & c & NA & NA & $\mathbf{N A}$ & NA & 0.0570 & 2 \\
\hline Total dissolved solids & NA & NA & 5220000 & 1264.0000 & NA & NA & 3180000 & 996.0000 \\
\hline Total suspended solids & 5.0000 & 64.0000 & 7.0000 & 2920000 & 4.0000 & 129.0000 & 4.0000 & 50.0000 \\
\hline Turbidity (NTUY & 6.0000 & 79.0000 & 4.05 & 180.0000 & 4.0500 & 300.0000 & 7.4000 & 1400.0000 \\
\hline Uranium & 0.0270 & 0.0470 & 0.0230 & 0.0350 & 0.0380 & 0.6300 & 0.0270 & 0.2070 \\
\hline Zinc & $<0.0200$ & 0.0420 & 0.0200 & 0.3200 & $<0.0200$ & 0.0620 & $<0.0200$ & 0.0750 \\
\hline
\end{tabular}

Located at Mitchell Branch kilometer (MIK) 0.12

NA $=$ not available

Less than (<) values were assigned if more than $50 \%$ of the initial observations bad (<) values.

One observation.

Two observations

NTU = nephelometric turbidity units.

Note: For information on sampling frequency and type, see Table A.8 in this report. 


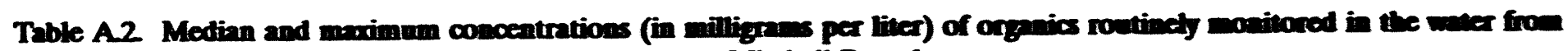
bover Miluchell Branch

\begin{tabular}{|c|c|c|c|c|c|c|c|c|}
\hline \multirow[b]{3}{*}{ Parameter } & \multicolumn{8}{|c|}{$K-1700$} \\
\hline & \multicolumn{2}{|c|}{1986} & \multicolumn{2}{|c|}{1987} & \multicolumn{2}{|c|}{1988} & \multicolumn{2}{|c|}{1989} \\
\hline & Median & $\operatorname{Max}$ & Median & $\operatorname{Max}$ & Median & Max & Median & $\operatorname{Max}$ \\
\hline 11 1-Trichloroethane & 4.0000 & 12.0000 & 2.0000 & 7.0000 & 2.0000 & 80000 & 20000 & 39.0000 \\
\hline 112 2-Tetrachloroethane & $<5.0000^{b}$ & 5.0000 & $<5.0000$ & $<5.0000$ & $<5.0000$ & $<5.0000$ & $<5.0000$ & $<5.0000$ \\
\hline 1 1-Dichloroethane & $<5.0000$ & 5.0000 & 3.0000 & 5.0000 & $<5.0000$ & 35.0000 & $<5.0000$ & 6.0000 \\
\hline 1 1-Dichloroethene & 5.0000 & 5.0000 & 20000 & 5.0000 & $<5.0000$ & 5.0000 & $<5.0000$ & 14.0000 \\
\hline 1 2-Dichloroethane & $<5.0000$ & $<5.0000$ & 5.0000 & 48.0000 & $<5.0000$ & $<5.0000$ & $<5.0000$ & $<5.0000$ \\
\hline 2-Butanone & $<10.0000$ & 16.0000 & 9.0000 & 20.0000 & 65.5000 & 100.0000 & $<10.0000$ & c \\
\hline 2-Hexanone & $<10.0000$ & 10.0000 & 20000 & 13.0000 & $\mathbf{N A}^{d}$ & NA & $<10.0000$ & c \\
\hline Acetone & 10.0000 & 25.0000 & 10.0000 & 200.0000 & 15.0000 & 48.0000 & $<10.0000$ & $c$ \\
\hline Acrolein & NA & NA & 5.0000 & 100.0000 & NA & NA & NA & NA \\
\hline Benzene & 5.0000 & 10.0000 & $<5.0000$ & $<5.0000$ & $<5.0000$ & 5.0000 & $<5.0000$ & 5.0000 \\
\hline Bromodichloromethane & $<5.0000$ & 5.0000 & $<5.0000$ & 5.0000 & $<5.0000$ & 5.0000 & $<5.0000$ & 5.0000 \\
\hline Bromoform & $<5.0000$ & 5.0000 & $<5.0000$ & $<5.0000$ & $<5.0000$ & $<5.0000$ & $<5.0000$ & $<5.0000$ \\
\hline Carbon tetrachloride & $<5.0000$ & 5.0000 & $<5.0000$ & $<5.0000$ & $<5.0000$ & 5.0000 & $<5.0000$ & $<5.0000$ \\
\hline Chlorobenzene & 5.0000 & 5.0000 & $<5.0000$ & $<5.0000$ & $<5.0000$ & 5.0000 & $<5.0000$ & 5.0000 \\
\hline Chloroethane & $<10.0000$ & 10.0000 & $<10.0000$ & 10.0000 & $<10.0000$ & $<10.0000$ & $<10.0000$ & 10.0000 \\
\hline Chloroform & 5.0000 & 9.0000 & 4.0000 & 14.0000 & 5.0000 & 17.0000 & 3.0000 & 10.0000 \\
\hline Chloromethane & $<10.0000$ & 10.0000 & $<10.0000$ & 10.0000 & $<10.0000$ & $<10.0000$ & $<10.0000$ & 10.0000 \\
\hline Cis-1 2-dichloroethene & NA & NA & NA & NA & 28.0000 & • & NA & NA \\
\hline Cis-1 3-dichloropropene & $<5.0000$ & 5.0000 & $<5.0000$ & $<5.0000$ & $<5.0000$ & $<5.0000$ & $<5.0000$ & $<5.0000$ \\
\hline Dibromochloromethane & $<5.0000$ & 5.0000 & $<5.0000$ & $<5.0000$ & $<5.0000$ & $<5.0000$ & $<5.0000$ & $<5.0000$ \\
\hline Dibromomethane & 1.0000 & 2.0000 & 37.0000 & c & NA & NA & NA & NA \\
\hline
\end{tabular}


Table A2 (comtined)

\begin{tabular}{|c|c|c|c|c|c|c|c|c|}
\hline \multirow[b]{3}{*}{ Parameter } & \multicolumn{8}{|c|}{$\mathrm{K}-1700^{\circ}$} \\
\hline & \multicolumn{2}{|c|}{1986} & \multicolumn{2}{|c|}{1987} & \multicolumn{2}{|c|}{1988} & \multicolumn{2}{|c|}{1989} \\
\hline & Median & $\operatorname{Max}$ & Median & Max & Median & $\operatorname{Max}$ & Median & $\operatorname{Max}$ \\
\hline Ethylbenzene & 5.0000 & 5.0000 & $<5.0000$ & $<5.0000$ & $<5.0000$ & 5.0000 & $<5.0000$ & $<5.0000$ \\
\hline $\begin{array}{l}\text { Freon } 113 \\
\text { Methylene chloride }\end{array}$ & $\begin{array}{l}3.0000 \\
5.0000\end{array}$ & $\begin{array}{l}5.0000 \\
5.0000\end{array}$ & $\begin{array}{l}2.0000 \\
5.0000\end{array}$ & $\begin{array}{r}920.0000 \\
6.0000\end{array}$ & $\begin{array}{r}7.0000 \\
<5.0000\end{array}$ & $\begin{array}{r}10.0000 \\
5.0000\end{array}$ & $\underset{<5.0000}{\text { NA }}$ & $\begin{array}{l}\text { NA } \\
5.0000\end{array}$ \\
\hline Tetrachloroethane & 5.0000 & 13.0000 & 2.0000 & 29.0000 & 2.0000 & 5.0000 & 2.0000 & 98.0000 \\
\hline Toluene & 2.0000 & 5.0000 & 1.0000 & 49.2000 & $<5.0000$ & 5.0000 & $<5.0000$ & 5.0000 \\
\hline Trans-1 2-dichloroethene & 40.0000 & 76.0000 & 43.0000 & 140.0000 & 38.5000 & 63.0000 & 30.0000 & 50.0000 \\
\hline Trans-1 3-dichloropropene & $<5.0000$ & 5.0000 & $<5.0000$ & $<5.0000$ & $<5.0000$ & $<5.0000$ & $<5.0000$ & 32.0000 \\
\hline Trichloroethane & 60.0000 & 86.0000 & 55.0000 & 510.0000 & 43.0000 & 87.0000 & 34.0000 & 720000 \\
\hline Vinyl chloride & 10.0000 & 13.0000 & 8.0000 & 17.0000 & 6.0000 & 10.0000 & 6.0000 & 10.0000 \\
\hline Xylenes, total & 2.0000 & 5.0000 & 1.0000 & 81.0000 & 1.0000 & 5.0000 & $<5.0000$ & $c$ \\
\hline
\end{tabular}

¿Located at Mitchell Branch kilometer (MIK) 0.12.

bess than values $(<)$ were assigned if more than $50 \%$ of the initial observations had $(<)$ values.

One observation.

NA $=$ Not available.

Two observations.

Note: For information on sampling frequency and type, see Table A8 in this report. 
Table A.3. Median and maximum concentrations (in milligrams per liter unless noted otherwise) of National Pollutant Discharge Elimination System parameters in the water from K-1407-B pond

\begin{tabular}{|c|c|c|c|c|c|c|}
\hline \multirow[b]{3}{*}{ Parameter } & \multicolumn{6}{|c|}{ K-1407-B } \\
\hline & \multicolumn{2}{|c|}{$1986^{\circ}$} & \multicolumn{2}{|c|}{$1987^{\circ}$} & \multicolumn{2}{|c|}{$1988^{4}$} \\
\hline & Median & $\operatorname{Max}$ & Median & $\operatorname{Max}$ & Median & $\operatorname{Max}$ \\
\hline Alpha, total (pCi/L) & $\mathbf{N A}^{\mathbf{e}}$ & NA & 7.0000 & 29.2000 & 5.0000 & J \\
\hline Aluminum, total & 0.2300 & 0.6200 & 0.2000 & 2.1000 & 0.2350 & 1.6000 \\
\hline Ammonia (as N) & $<0.2000$ & 0.5000 & $<0.2000$ & 0.7400 & $<0.2000$ & 2.9000 \\
\hline Antimony, total & $<0.0500$ & 0.0620 & $<0.0500$ & 0.1300 & $<0.0500$ & 0.2700 \\
\hline Anenic, total & 0.0050 & 0.0070 & $<0.0050$ & 0.0100 & 0.0050 & 0.0210 \\
\hline Barium, total & $<0.1000$ & $<0.1000$ & $<0.1000$ & $<0.1000$ & $<0.1000$ & $<0.1000$ \\
\hline Beryllium, total & $<0.0010$ & $<0.0010$ & $<0.0010$ & $<0.0010$ & $<0.0010$ & 0.0011 \\
\hline Beta, total (pCi/L) & 78.0000 & 93.0000 & 16.1000 & 52.9000 & 19.0000 & J \\
\hline Boron, total & 0.1200 & 0.1600 & 0.0765 & 0.1900 & 0.1050 & 12.0000 \\
\hline Bromide & 3.0000 & 6.0000 & $<2.0000$ & 3.0000 & $<2.0000$ & 3.7000 \\
\hline Cadmium & $<0.0020$ & $<0.0020$ & $<0.0020$ & 0.0500 & $<0.0020$ & 0.0250 \\
\hline Cesium & $<100.0000$ & $n$ & $<100.0000$ & $<100.0000$ & 0.0000 & 100.0000 \\
\hline Chemical axygen demand & 14.0000 & 66.0000 & 11.0000 & 146.0000 & 16.0000 & 56.0000 \\
\hline Chloride & 255.0000 & 366.0000 & 288.0000 & 1095.0000 & 476.5000 & 994.0000 \\
\hline Chlorine, total residual & $<0.1000$ & $<0.1000$ & $<0.1000$ & 0.5000 & $<0.1000$ & 0.7000 \\
\hline Chromium & $<0.0100$ & 0.0130 & $<0.0100$ & 1.9000 & $<0.0100$ & 0.0210 \\
\hline Cobalt, total & $<0.1000$ & 0.1800 & $<0.1000$ & $<0.1000$ & $<0.1000$ & $<0.1000$ \\
\hline Copper & 0.0042 & 0.0910 & $<0.0040$ & 2.0000 & 0.0043 & 0.0450 \\
\hline Cyanide & 0.0060 & $0.00 \%$ & $<0.0020$ & 0.1000 & 0.0060 & 0.1980 \\
\hline Flow (L/s) & NA & NA & NA & NA & 1.3000 & 165.5000 \\
\hline Fluoride & 0.3500 & 3.0000 & 0.8000 & 21.0000 & 2.5000 & .57 .0000 \\
\hline Iron, total & 1.5000 & 3.8000 & 0.4350 & 5.0000 & 0.3800 & 7.0000 \\
\hline Kjeldahl nitrogen & 0.6000 & 1.3000 & 0.6000 & 1.6200 & 0.8500 & 4.4200 \\
\hline Lead & $<0.0040$ & 0.0070 & $<0.0040$ & 0.0680 & $<0.0093$ & 0.2100 \\
\hline Magnesium, total & 23.0000 & 27.0000 & 15.5000 & 35.0000 & 20.0000 & 27.0000 \\
\hline Manganese, total & 0.1800 & 0.2700 & 0.0560 & 2.0000 & 0.0580 & 0.6300 \\
\hline Mercury, total & $<0.0002$ & $<0.0002$ & $<0.0002$ & 0.0010 & $<0.0002$ & 0.0023 \\
\hline Molybdenum, total & $<0.0100$ & 0.0140 & $<0.0100$ & 0.0250 & $<0.0100$ & 0.0360 \\
\hline Neptunium & $<1.0000$ & $n$ & $<1.0000$ & $<1.0000$ & 0.2650 & 1.0000 \\
\hline Nickel & 0.2300 & 0.8600 & $<0.0500$ & 2.0000 & $<0.0500$ & 1.6000 \\
\hline Nitrate (as N) & 0.6300 & 22.3000 & 0.5500 & 26.2000 & $<0.5500$ & 15.0000 \\
\hline Nitrogen (total organic) & NA & NA & NA & NA & 0.9500 & 1.4000 \\
\hline Oil and grease & $<2.0000$ & 3.0000 & $<2.000$ & 3.0000 & $<2.0000$ & 51.0000 \\
\hline $\mathrm{pH}$ (pH units) & NA & NA & 8.1000 & 8.4000 & 7.9000 & 8.9000 \\
\hline Phenols, total & 0.0010 & 0.0040 & $<0.0010$ & 0.0250 & 0.0025 & 0.0300 \\
\hline Phosphorus, total & NA & NA & $<0.2000$ & 1.4000 & $<0.2000$ & 8.4000 \\
\hline Plutonium & $<1.0000$ & $n$ & $<1.0000$ & 1.4000 & -0.0200 & 1.0000 \\
\hline \multicolumn{7}{|c|}{ Polychlorinated biphenyls $(\mu g / L)$} \\
\hline Aroclor-1254 & $\mathrm{BD}^{\prime}$ & BD & $<1.0000$ & 1.2000 & $<1.0000$ & 1.9000 \\
\hline
\end{tabular}


Tabie A.3 (continued)

\begin{tabular}{|c|c|c|c|c|c|c|}
\hline \multirow[b]{3}{*}{ Parameter } & \multicolumn{6}{|c|}{ K-1407-Ba } \\
\hline & \multicolumn{2}{|c|}{$1986^{\circ}$} & \multicolumn{2}{|c|}{$1987^{r}$} & \multicolumn{2}{|c|}{$1988^{4}$} \\
\hline & Median & $\operatorname{Max}$ & Median & $\operatorname{Max}$ & Median & Max \\
\hline Selenium, total & $<0.0500$ & 0.0500 & $<0.0050$ & $<0.0050$ & $<0.0050$ & $<0.0050$ \\
\hline Silver & $<0.0100$ & $<0.0100$ & $<0.0100$ & 0.0140 & $<0.0100$ & 0.0210 \\
\hline Sulfate (as $\mathrm{SO}_{4}$ ) & 585.0000 & 859.0000 & 339.0000 & 752.0000 & 507.0000 & 1410.0000 \\
\hline Sulfide (as S) & $<1.0000$ & $<1.0000$ & 1.0000 & 1.0000 & $<1.0000$ & 2.0000 \\
\hline Sulfite (as $\mathrm{SO}_{3}$ ) & $<2.0000$ & 3.0000 & 2.0000 & 7.0000 & $<2.0000$ & 4.0000 \\
\hline Technetium (pCi/L) & 276.5000 & 444.0000 & $<214.5000$ & 327.0000 & 222.0000 & 3004.0000 \\
\hline Temperature $\left({ }^{\circ} \mathrm{C}\right)$ & NA & NA & NA & NA & 23.0000 & 33.0000 \\
\hline Thallium, total & $<0.0100$ & $<0.0100$ & $<0.0100$ & $<0.0100$ & $<0.0100$ & 0.0100 \\
\hline Tin, total & $<0.0100$ & $<0.0100$ & $<0.0100$ & 0.0150 & $<0.0100$ & 0.0280 \\
\hline Titanium, total & $<0.0030$ & 0.0093 & $<0.0030$ & 0.0230 & $<0.0030$ & 0.0280 \\
\hline Total dissolved solids & 1594.0000 & 1922.0000 & 1196.0000 & 3204.0000 & 1680.0000 & 14032.0000 \\
\hline Total organic carbon & 19.0000 & 54.0000 & 4.3000 & 63.0000 & 11.8500 & 43.0000 \\
\hline Total suspended solids & 12.5000 & 398.0000 & 21.0000 & 21.0000 & 9.0000 & 52.0000 \\
\hline Uranium & 0.0510 & 0.1620 & 0.0455 & 0.1820 & 0.1900 & 4.9400 \\
\hline Zinc & 0.0240 & 0.0320 & $<0.0200$ & 2.0000 & $<0.0200$ & 0.1100 \\
\hline
\end{tabular}

a holding pond.

Sampling period November-December.

'Sampling period March-December, January and February not available.

SSampling does not include January or October.

NA $=$ not available.

One observation.

Less than values $(<)$ were assigned if more than $50 \%$ of the initial parameters had $(<)$ values.

"Two observations.

'BD $=$ below detection limit of $<1.0 \mathrm{ug} / \mathrm{L}$.

Note: For information concerning sampling frequency and type, see Table A.8 in this report. 
Table A.4. Median and maximum concentrations (in milligrams per liter) of organics routinely monitored in the water from $\mathrm{K}-1407-\mathrm{B}$ pond

\begin{tabular}{|c|c|c|c|c|c|c|}
\hline \multirow[b]{3}{*}{ Parameter } & \multicolumn{6}{|c|}{ K-1407-Ba } \\
\hline & \multicolumn{2}{|c|}{1986} & \multicolumn{2}{|c|}{1987} & \multicolumn{2}{|c|}{1988} \\
\hline & Median & $\operatorname{Max}$ & Median & Max & Median & $\operatorname{Max}$ \\
\hline 11 1-Trichloroethane & 2.0000 & 62.0000 & $<5.0000$ & 25.0000 & 5.0000 & 16.0000 \\
\hline 112 2-Tetrachloroethane & $<5.0000^{\circ}$ & 50.0000 & $<5.0000$ & $<5.0000$ & $<5.0000$ & $<5.0000$ \\
\hline 11-Dichloroethane & $<5.0000$ & 50.0000 & $<5.0000$ & 5.0000 & $<5.0000$ & 9.0000 \\
\hline 1 1-Dichloroethene & $<5.0000$ & 50.0000 & $<5.0000$ & 5.0000 & $<5.0000$ & 7.0000 \\
\hline 1 2-Dichloroethane & $<5.0000$ & 50.0000 & $<5.0000$ & 5.0000 & $<5.0000$ & $<5.0000$ \\
\hline 2-Butannone & $<10.0000$ & 100.0000 & 10.0000 & 34.0000 & 8.0000 & 20.0000 \\
\hline 2-Hexanone & $<10.0000$ & 100.0000 & 1.0000 & 6.0000 & 7.0000 & c \\
\hline Acetone & 20.0000 & 550.0000 & 26.0000 & 1200.0000 & 35.5000 & 610.0000 \\
\hline Acrolein & NA & NA & $<5.0000$ & 100.0000 & NA & NA \\
\hline Benzene & 3.5000 & 50.0000 & $<5.0000$ & 8.0000 & $<5.0000$ & 5.0000 \\
\hline Bromodichloromethane & $<5.0000$ & 50.0000 & $<5.0000$ & $<5.0000$ & $<5.0000$ & 5.0000 \\
\hline Bromoform & $<5.0000$ & 50.0000 & $<5.0000$ & 5.0000 & $<5.0000$ & 5.0000 \\
\hline Carbon tetrachloride & $<5.0000$ & 50.0000 & $<5.0000$ & 5.0000 & $<5.0000$ & $<5.0000$ \\
\hline Chlorobenzene & 5.0000 & 50.0000 & $<5.0000$ & 5.0000 & $<5.0000$ & 5.0000 \\
\hline Chloroethane & $<10.0000$ & 100.0000 & $<10.0000$ & 10.0000 & 10.0000 & 10.0000 \\
\hline Chloroform & 5.0000 & 50.0000 & 4.0000 & 20.0000 & 2.0000 & 5.0000 \\
\hline Chloromethane & $<10.0000$ & 100.0000 & $<10.0000$ & 10.0000 & $<10.0000$ & 10.0000 \\
\hline Cis-1 2-dichloroethene & NA & NA & NA & NA & 8.0000 & 10.0000 \\
\hline Cis-1 3-dichloropropene & $<5.0000$ & 50.0000 & $<5.0000$ & $<5.0000$ & $<5.0000$ & 5.0000 \\
\hline Dibromochloromethane & $<5.0000$ & 50.0000 & $<5.0000$ & $<5,0000$ & $<5.0000$ & $<5.0000$ \\
\hline Dibromomethane & 1.0000 & 2.0000 & 1.0000 & • & NA & NA \\
\hline Ethylbenzene & 5.0000 & 50.0000 & $<5.0000$ & $<5.0000$ & $<5.0000$ & 5.0000 \\
\hline Freon 113 & 2.0000 & 6.0000 & 2.0000 & 18.0000 & 5.0000 & 5.0000 \\
\hline Methylene chloride & $<5.0000$ & 50.0000 & 5.0000 & 240.0000 & $<5.0000$ & 5.0000 \\
\hline Tetrachloroethane & 18.0000 & 120.0000 & 3.0000 & 23.0000 & $<5.0000$ & 12.0000 \\
\hline Toluene & 4.0000 & 50.0000 & 3.0000 & 5.0000 & $<5.0000$ & 5.0000 \\
\hline Trans-1 2-dichloroethene & 11.0000 & 50.0000 & 7.0000 & 120.0000 & 11.0000 & 240.0000 \\
\hline Trans-1 3-dichloropropene & $<5.0000$ & 50.0000 & $<5.0000$ & $<5.0000$ & $<5.0000$ & $<5.0000$ \\
\hline Trichloroethane & 33.5000 & 56.0000 & 14.0000 & 50.0000 & 12.0000 & 91.0000 \\
\hline Vinyl chloride & $<10.0000$ & 100.0000 & $<10.0000$ & 10.0000 & $<10.0000$ & 17.0000 \\
\hline Xylenes, total & 3.0000 & 50.0000 & 1.0000 & 43.0000 & 1.0000 & - \\
\hline
\end{tabular}

\footnotetext{
a A holding pond.

${ }^{b}$ Less than values $(<)$ were assigned if more than $50 \%$ of the initial observations had $(<)$ values.

Two observations.

${ }^{d} \mathrm{NA}=$ Not available.

'One observation.

Note: For information concerning sampling frequency and type, see Table A.8 in this report.
} 
Table A.5. Median and marimum concentrations (in milligrams per liter unless noted otherwise) of National Pollutant Discharge Elimination System parameters in the water from K-1407-E/F pond

\begin{tabular}{|c|c|c|c|c|}
\hline \multirow[b]{3}{*}{ Parameter } & \multicolumn{4}{|c|}{ K-1407-E/F" } \\
\hline & \multicolumn{2}{|c|}{1988} & \multicolumn{2}{|c|}{1989} \\
\hline & Median & $\operatorname{Max}$ & Median & $\operatorname{Max}$ \\
\hline Aluminum, total & $<0.1000$ & 0.4700 & $<0.1000$ & 0.5500 \\
\hline Antimony, total & $<0.0500$ & e & $N^{d}$ & NA \\
\hline Arsenic, total & $<0.0050$ & • & $<0.0050$ & $<0.0050$ \\
\hline Barium, total & $<0.1000$ & $<0.1000$ & $<0.1000$ & $<0.1000$ \\
\hline Beryllium, total & $<0.0010$ & $<0.0010$ & $<0.0010$ & $<0.0010$ \\
\hline Boron, total & 0.0750 & 0.1500 & 0.0580 & 0.1300 \\
\hline Cadmium & $<0.0030$ & $<0.0030$ & $<0.0020$ & 0.0030 \\
\hline Chromium & $<0.0100$ & $<0.0100$ & $<0.0100$ & 0.1000 \\
\hline Cobalt, total & $<0.1000$ & $<0.1000$ & $<0.1000$ & $<0.1000$ \\
\hline Copper & $<0.0040$ & 0.0130 & 0.0160 & 0.0640 \\
\hline Flow (LS) & 2.7523 & 5.8372 & 2.1403 & 12.1352 \\
\hline Iron, total & 0.6800 & 0.9700 & 0.5100 & 4.7000 \\
\hline Lead & 0.0270 & 0.0500 & $<0.0040$ & 0.0500 \\
\hline Magnesium, total & 18.0000 & 63.0000 & 12.0000 & 24.0000 \\
\hline Manganese, total & 0.1350 & 0.2000 & 0.0835 & 0.1400 \\
\hline Mercury, total & $<0.0002$ & c & NA & NA \\
\hline Molybdenum, total & $<0.0100$ & $<0.0100$ & $<0.0100$ & $<0.0100$ \\
\hline Nickel & $<0.0500$ & $<0.0500$ & $<0.0500$ & 6.3000 \\
\hline Oil and grease & $<2.0000$ & $<2.0000$ & $<2.0000$ & 2.7000 \\
\hline pH (pH units) & 8.0000 & 9.1000 & 7.8000 & 11.5000 \\
\hline Phosphorus, total & $<0.2000$ & $<0.2000$ & $<0.2000$ & $:$ \\
\hline Selenium, total & $<0.0050$ & $\cdot$ & $<0.0050$ & $<0.0050$ \\
\hline Silver & $<0.0100$ & $<0.0100$ & $<0.0100$ & 0.1000 \\
\hline Sulfate (as $\mathrm{SO}_{4}$ ) & 914.0000 & 1100.0000 & 634.0000 & 1710.0000 \\
\hline Temperature & 8.4000 & 14.7000 & 16.9000 & 33.3000 \\
\hline Titanium, total & 0.0110 & 0.0150 & $<0.0030$ & 0.0180 \\
\hline Total suspended solids & 7.0000 & 10.0000 & 8.0000 & 65.0000 \\
\hline Turbidity (NTUY & NA & NA & 2.4500 & 3.3000 \\
\hline Zinc & $<0.0200$ & 0.0260 & $<0.0200$ & 0.3200 \\
\hline
\end{tabular}

"Holding ponds.

'Less than (<) values were assigned if more than $50 \%$ of the initial observations had (<) values.

'One observation.

NA = not available.

Two observations.

Nephelometric turbidity units.

Note: For information concerning sampling frequency and type, see Table A.8 in this report. 
Table A.6. Median and maximum concentrations (in milligrams per liter unless noted otherwise) of National Pollutant Discharge Elimination System parameters in the water from $\mathrm{K}-1407-\mathrm{J}$

\begin{tabular}{|c|c|c|c|c|c|c|}
\hline \multirow[b]{3}{*}{ Parameter } & \multicolumn{6}{|c|}{$\mathrm{K}-1407-\mathrm{J}^{a}$} \\
\hline & \multicolumn{2}{|c|}{ Nov-Dec 1988} & \multicolumn{2}{|c|}{ Nov-Dec 1989} & \multicolumn{2}{|c|}{ Year 1989} \\
\hline & Median & $\operatorname{Max}$ & Median & $\operatorname{Max}$ & Median & $\operatorname{Max}$ \\
\hline Alpha, total (pCi/L) & $\mathbf{N A}^{b}$ & NA & 320.0000 & c & 247.0000 & 580.0000 \\
\hline Aluminum, total & 0.2000 & 0.7400 & 0.4200 & 2.3000 & 0.2850 & 15.0000 \\
\hline Ammonia (as N) & $<0.2000^{d}$ & $<0.2000$ & $<0.2000$ & $\cdot$ & $<0.2000$ & $<0.2000$ \\
\hline Antimony, total & $<0.0500$ & 0.0880 & $<0.0500$ & $<0.0500$ & $<0.0500$ & $<0.0500$ \\
\hline Arsenic, total & 0.0054 & 0.0063 & $<0.0050$ & 0.0110 & $<0.0050$ & 0.1500 \\
\hline Barium, total & $<0.1000$ & $<0.1000$ & $<0.1000$ & $<0.1000$ & $<0.1000$ & 0.1400 \\
\hline Beryllium, total & $<0.0010$ & 0.0024 & $<0.0010$ & $<0.0010$ & $<0.0010$ & 0.0016 \\
\hline Beta, total $(\mathrm{pCi} / \mathrm{L})$ & NA & NA & 682.0000 & c & 271.0000 & 1349.0000 \\
\hline Boron, total & 0.0940 & 1.1000 & 0.0655 & 0.1100 & 0.1200 & 1.1000 \\
\hline Bromide & $<2.0000$ & $<2.0000$ & $<2.0000$ & $<2.0000$ & $<2.0000$ & $<2.0000$ \\
\hline Cadmium & $<0.0020$ & 0.0048 & $<0.0020$ & 0.0086 & $<0.0020$ & 0.0086 \\
\hline Cesium & NA & NA & 0.0000 & c & 0.0000 & 0.0000 \\
\hline Chemical oxygen demand & 9.0000 & 46.0000 & 13.5000 & 28.0000 & 14.0000 & 157.0000 \\
\hline Chloride & 905.0000 & 1740.0000 & 128.0000 & 153.0000 & 183.0000 & 356.0000 \\
\hline Chlorine, total residual & 0.1000 & 47.0000 & 0.0200 & 0.1400 & $<0.0200$ & 0.0600 \\
\hline Chromium & 0.0110 & 0.0410 & $<0.0100$ & 0.0740 & $<0.0100$ & 0.4400 \\
\hline Cobalt, total & $<0.1000$ & $<0.1000$ & $<0.1000$ & $<0.1000$ & $<0.1000$ & $<0.1000$ \\
\hline Copper & 0.0420 & 0.4100 & 0.0175 & 0.0910 & 0.0215 & 0.4900 \\
\hline Cyanide & 0.0090 & 0.0240 & $<0.1000$ & 0.1000 & 0.0030 & 0.1000 \\
\hline Flow (L/s) & 1.5000 & 2.0000 & 0.5000 & 5.4000 & 3.7000 & 6.0000 \\
\hline Fluoride & 25.0000 & 124.0000 & 1.3000 & 2.1000 & 16.0000 & 95.0000 \\
\hline Iron, total & 2.1000 & 6.5000 & 0.4950 & 7.9000 & 0.8650 & 42.0000 \\
\hline Kjeldahl nitrogen & 0.3900 & 0.7300 & 0.8000 & 1.0000 & 0.8000 & 3.6000 \\
\hline Lead & 0.0185 & 0.0370 & 0.0046 & 0.1290 & 0.0047 & 0.1290 \\
\hline Magnesium, total & 16.0000 & 21.0000 & 15.0000 & 20.0000 & 15.0000 & 30.0000 \\
\hline Manganese, total & 0.0850 & 0.1500 & 0.0635 & 0.3500 & 0.1050 & 0.6100 \\
\hline Mercury, total & 0.0002 & 0.0010 & 0.0004 & 0.0065 & $0.00 ? 2$ & 0.0590 \\
\hline Molybdenum, total & 0.0120 & 0.0380 & $<0.0100$ & 0.0400 & $<0.0101$ & 0.0400 \\
\hline Neptunium & NA & NA & 1.0000 & c & $0.66 \% 0$ & 1.3000 \\
\hline Nickel & 0.0650 & 0.1500 & 0.1700 & 0.8500 & 0.1350 & 2.6000 \\
\hline Nitrate (as $\mathrm{N}$ ) & 1.2000 & 2.4000 & 0.5000 & 3.0000 & 0.6000 & 1390.0000 \\
\hline Oil and grease & $<2.0000$ & $<2.0000$ & $<2.0000$ & $<2.0000$ & $<2.0000$ & $<2.0000$ \\
\hline $\mathrm{pH}$ (pH units) & 8.3500 & 9.3000 & 8.2000 & 8.7000 & 8.0000 & 9.3000 \\
\hline Phenols, total & $<0.0300$ & 0.0300 & $<0.0100$ & 0.0600 & $<0.0100$ & 0.0600 \\
\hline Phosphorus, total & $<0.2000$ & 0.8000 & 0.7400 & 2.0000 & 0.4050 & 9.3000 \\
\hline Plutonium & NA & NA & 0.2400 & c & 0.0900 & 1.8500 \\
\hline Selenium, total & $<0.0050$ & $<0.0050$ & $<0.0050$ & $<0.0050$ & $<0.0050$ & $<0.0050$ \\
\hline
\end{tabular}


Table A.6 (continued)

\begin{tabular}{|c|c|c|c|c|c|c|}
\hline \multirow[b]{3}{*}{ Parameter } & \multicolumn{6}{|c|}{ K-1407-Ja } \\
\hline & \multicolumn{2}{|c|}{ Nov-Dec 1988} & \multicolumn{2}{|c|}{ Nov-Dec 1989} & \multicolumn{2}{|c|}{ Year 1989} \\
\hline & Median & $\operatorname{Max}$ & Median & $\operatorname{Max}$ & Median & $\operatorname{Max}$ \\
\hline Silver & $<0.0100$ & $<0.0100$ & $<0.0100$ & 0.0100 & $<0.0100$ & 0.0100 \\
\hline Sulfate (as $\mathrm{SO}_{4}$ ) & 654.0000 & 1200.0000 & 307.5000 & 471.0000 & 447.0000 & 1500.0000 \\
\hline Sulfide (as S) & $<1.0000$ & 2.0000 & $<1.0000$ & $<1.0000$ & $<1.0000$ & $<1.000$ \\
\hline Sulfite (as $\mathrm{SO}_{3}$ ) & $<2.0000$ & $<2.0000$ & $<2.0000$ & $<2.0000$ & $<2.0000$ & $<2.0000$ \\
\hline Technetium, pCi/L & NA & NA & 1304.0000 & c & 526.0000 & 1719.0000 \\
\hline Temperature & 9.3000 & 100.0000 & 7.8000 & 11.5000 & 13.1000 & 31.9000 \\
\hline Thallium, total & $<0.0100$ & $<0.0100$ & $<0.0100$ & $<0.01 .00$ & $<0.0100$ & $<0.0100$ \\
\hline Tin, total & $<0.0100$ & 0.0100 & $<0.0100$ & 0.0100 & $<0.0100$ & 0.0310 \\
\hline Titanium, total & 0.0150 & 0.0440 & 0.0080 & 0.0560 & 0.0100 & 0.2200 \\
\hline Total dissolved solids & 2619.0000 & 6924.0000 & 720.0000 & 1604.0000 & 1358.0000 & 4096.0000 \\
\hline Total organic carbon & 12.0000 & 120.0000 & 5.0000 & 8.0000 & 8.0000 & 20.0000 \\
\hline Total suspended solids & 11.5000 & 23.0000 & 9.0000 & 118.0000 & 9.0000 & 359.0000 \\
\hline Uranium & 1.0950 & 2.8000 & 0.6380 & 0.9070 & 0.3380 & 0.5940 \\
\hline Zinc & 0.0500 & 0.4600 & 0.0360 & 0.2000 & 0.0465 & 0.3600 \\
\hline
\end{tabular}

A basin.

$\mathbf{N A}=$ not available.

'One observation.

Less than values $(<)$ were assigned if more than $50 \%$ of the initial observations had $(<)$ values.

Two observations.

SIE.

Note: For information concerning sampling frequency and type, see Table A.8 in this report. 
Table A.7. Median and maximum concentrations (in milligrams per liter unless noted otherwise) of organics routinely monitored in the water from K-1407-J

\begin{tabular}{|c|c|c|c|c|c|c|}
\hline \multirow[b]{3}{*}{ Parameter } & \multicolumn{6}{|c|}{ K-1407.J" } \\
\hline & \multicolumn{2}{|c|}{ Nov-Dec 1988} & \multicolumn{2}{|c|}{ Nov-Dec 1989} & \multicolumn{2}{|c|}{ Year 1989} \\
\hline & Median & $\operatorname{Max}$ & Median & $\operatorname{Max}$ & Median & $\operatorname{Max}$ \\
\hline 11 1-Trichloroethane & $<5.0000^{b}$ & 5.0000 & 6.0000 & 140.0000 & $<5.0000$ & 140.0000 \\
\hline 112 2-Tetrachloroethane & $<5.0000$ & $<5.0000$ & $<5.0000$ & 5.0000 & $<5.0000$ & 5.0000 \\
\hline 1 1-Dichloroethane & $<5.0000$ & 5.0000 & $<5.0000$ & 20.0000 & $<5.0000$ & 36.0000 \\
\hline 1 1-Dichloroethene & $<5.0000$ & $<5.0000$ & 5.0000 & 100.0000 & $<5.0000$ & 100.0000 \\
\hline 1 2-Dichloroethane & $<5.0000$ & $<5.0000$ & $<5.0000$ & $<5.0000$ & $<5.0000$ & $<5.0000$ \\
\hline Benzene & $<5.0000$ & $<5.0000$ & $<5.0000$ & $<5.0000$ & $<5.0000$ & $<5.0000$ \\
\hline Bromodichloromethane & $<5.0000$ & 5.0000 & $<5.0000$ & 5.0000 & $<5.0000$ & 5.0000 \\
\hline Bromoform & $<5.0000$ & $<5.0000$ & $<5.0000$ & $<5.0000$ & $<5.0000$ & $<5.0000$ \\
\hline Carbon tetrachloride & $<5.0000$ & 19.0000 & $<5.0000$ & 5.0000 & $<5.0000$ & 5.0000 \\
\hline Chlorobenzene & $<5.0000$ & $<5.0000$ & $<5.0000$ & $<5.0000$ & $<5.0000$ & 5.0000 \\
\hline Chloroethane & $<10.0000$ & $<10.0000$ & $<10.0000$ & $<10.0000$ & $<10.0000$ & $<10.0000$ \\
\hline Chloroform & 5.0000 & 13.0000 & 5.0000 & 11.0000 & 3.0000 & 11.0000 \\
\hline Chloromethane & $<10.0000$ & $<10.0000$ & $<10.0000$ & $<10.0000$ & $<10.0000$ & $<10.0000$ \\
\hline Cis-1 3-dichloropropene & $<5.0000$ & $<5.0000$ & $<5.0000$ & $<5.0000$ & $<5.0000$ & $<5.0000$ \\
\hline Dibromochloromethane & $<5.0000$ & $<5.0000$ & $<5.0000$ & $<5.0000$ & $<5.0000$ & $<5.0000$ \\
\hline Ethylbenzene & $<5.0000$ & $<5.0000$ & $<5.0000$ & $<5.0000$ & $<5.0000$ & 5.0000 \\
\hline Methylene chloride & $<5.0000$ & 5.0000 & $<5.0000$ & 5.0000 & $<5.0000$ & 8.0000 \\
\hline Tetrachloroethane & 13.0000 & 96.0000 & 11.0000 & 240.0000 & 5.0000 & 240.0000 \\
\hline Toluene & $<5.0000$ & 5.0000 & $<5.0000$ & 5.0000 & $<5.0000$ & 5.0000 \\
\hline Trans-1 2-dichloroethene & $<5.0000$ & 6.0000 & 22.0000 & 200.0000 & $<5.0000$ & 200.0000 \\
\hline Trans-1 3-dichloropropene & $<5.0000$ & $<5.0000$ & $<5.0000$ & $<5.0000$ & $<5.0000$ & $<5.0000$ \\
\hline Trichloroethane & 3.0000 & 17.0000 & 60.0000 & 1400.0000 & 5.0000 & 1400.0000 \\
\hline Vinyl chloride & $<10.0000$ & $<10.0000$ & $<10.0000$ & 12.0000 & $<10.0000$ & 12.0000 \\
\hline
\end{tabular}

a A basin.

'Less than values (<) were assigned if more than $50 \%$ of the initial observations had (<) values. Note: For information concerning sampling frequency and type, see Table A.8 in this report. 
Table A.8. Sampling frequency and sample type for regularly monitored water quality parameters as outlined

in the National Pollutant Discharge

Elimination System Permit

\begin{tabular}{|c|c|c|}
\hline Parameter & Sampling frequency" & Sample type ${ }^{b}$ \\
\hline Acid compounds & 5 & 3 \\
\hline Alpha, total & 5 & 4 \\
\hline Aluminum, total & 2 & 1 \\
\hline Ammonia (as $\mathbf{N}$ ) & 1 & 1 \\
\hline Antimony, total & 2 & 1 \\
\hline Arsenic, total & 1 & 1 \\
\hline Barium, total & 2 & 1 \\
\hline Base/neutral & 5 & 3 \\
\hline Beryllium, total & 2 & 1 \\
\hline Beta, total & 5 & 4 \\
\hline Boron, total & 2 & 1 \\
\hline Bromide & 1 & 1 \\
\hline Cadmium & 2 & $?$ \\
\hline Cesium & 5 & 4 \\
\hline Chemical oxygen demand & 3 & 1 \\
\hline Chloride & 1 & 1 \\
\hline Chlorine, total residual & 1 & 1 \\
\hline Chromium & 2 & 1 \\
\hline Cobalt, total & 2 & 1 \\
\hline Copper & 1 & 1 \\
\hline Cyanide & 1 & 2 \\
\hline Flow & 6 & $\mathbf{N A}^{c}$ \\
\hline Fluoride & 3 & 1 \\
\hline Iron, total & 2 & 1 \\
\hline Lead & 2 & 1 \\
\hline Magnesium, total & 2 & 1 \\
\hline Manganese, total & 2 & 1 \\
\hline Mercury, total & 2 & 1 \\
\hline Molybdenum, total & 2 & 1 \\
\hline Neptunium & 5 & 4 \\
\hline Nickel & 1 & 1 \\
\hline Nitrate (as $\mathrm{N}$ ) & 2 & 1 \\
\hline Nitrogen, total organic & 1 & 1 \\
\hline Oil and grease & 2 & 2 \\
\hline
\end{tabular}


Table A.8 (continued)

Parameter

Sampling frequency Sample type

pH

Pheriols, total

Phosphorus, total

Plutonium

Polychlorinated biphenyls

Selenium, total

Silver

Sulfate (as $\mathrm{SO}_{4}$ )

Sulfide (as $\mathbf{S}$ )

Sulfite (as $\left.\mathrm{SO}_{3}\right)$

Surfactants

Technetium

Temperature

Thallium, total

Tin, total

Titanium, total

Total dissolved solids

Total organic carbon

Total suspended solids

Turbidity

Uranium

Volatile compounds

Zinc

\begin{tabular}{ll}
6 & 2 \\
1 & 2 \\
1 & 1 \\
5 & 4 \\
1 & 1 \\
2 & 1 \\
1 & 1 \\
1 & 1 \\
1 & 1 \\
1 & 1 \\
1 & 1 \\
5 & 4 \\
3 & 2 \\
1 & 1 \\
1 & 1 \\
2 & 1 \\
3 & 1 \\
2 & 1 \\
3 & 1 \\
3 & 2 \\
1 & 4 \\
4 & 2 \\
2 & 1 \\
\hline
\end{tabular}

${ }^{a} 1=$ once per week; 2 = twice per week; $3=$ four times per week; 4 = five times per week; $5=$ monthly; and $6=$ continuous sampling.

${ }^{b 1}=24 \mathrm{~h}$ per composite; $2=$ grab; $3=72 \mathrm{~h}$ grab per composite; 4 = once per week; $24 \mathrm{~h}$ composite was analyzed for uranium.

(NA) Not applicable.

Note: The weekly composites were compiled into a monthly sample and analyzed for $\mathrm{Cs}, \mathrm{Pu}, \mathrm{Np}$, and $\mathrm{Tc}$. Isotopic analysis was conducted on uranium any week that values were above $0.02 \mathrm{mg} / \mathrm{L}$. 


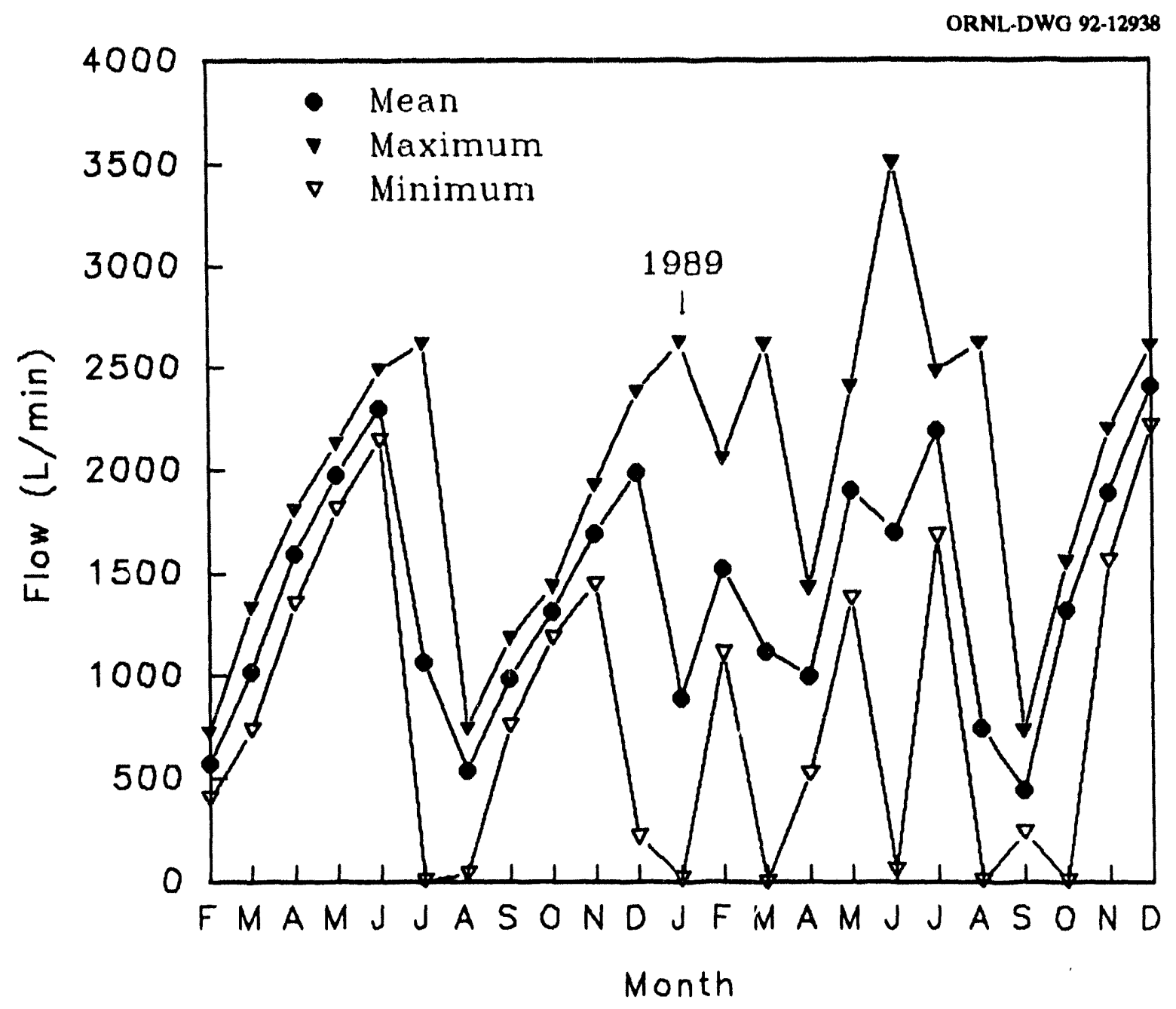

Fig. A.1. Mean monthly streamflow for Mitchell Branch at Mitchell Branch kilometer 0.12 (National Pollutant Discharge Elimination System monitoring station K-1700) below Oak Ridge K-25 Site. Maximum and minimum values for each month are also shown. Source: National Pollutant Discharge Elimination System Monthly Reports. 

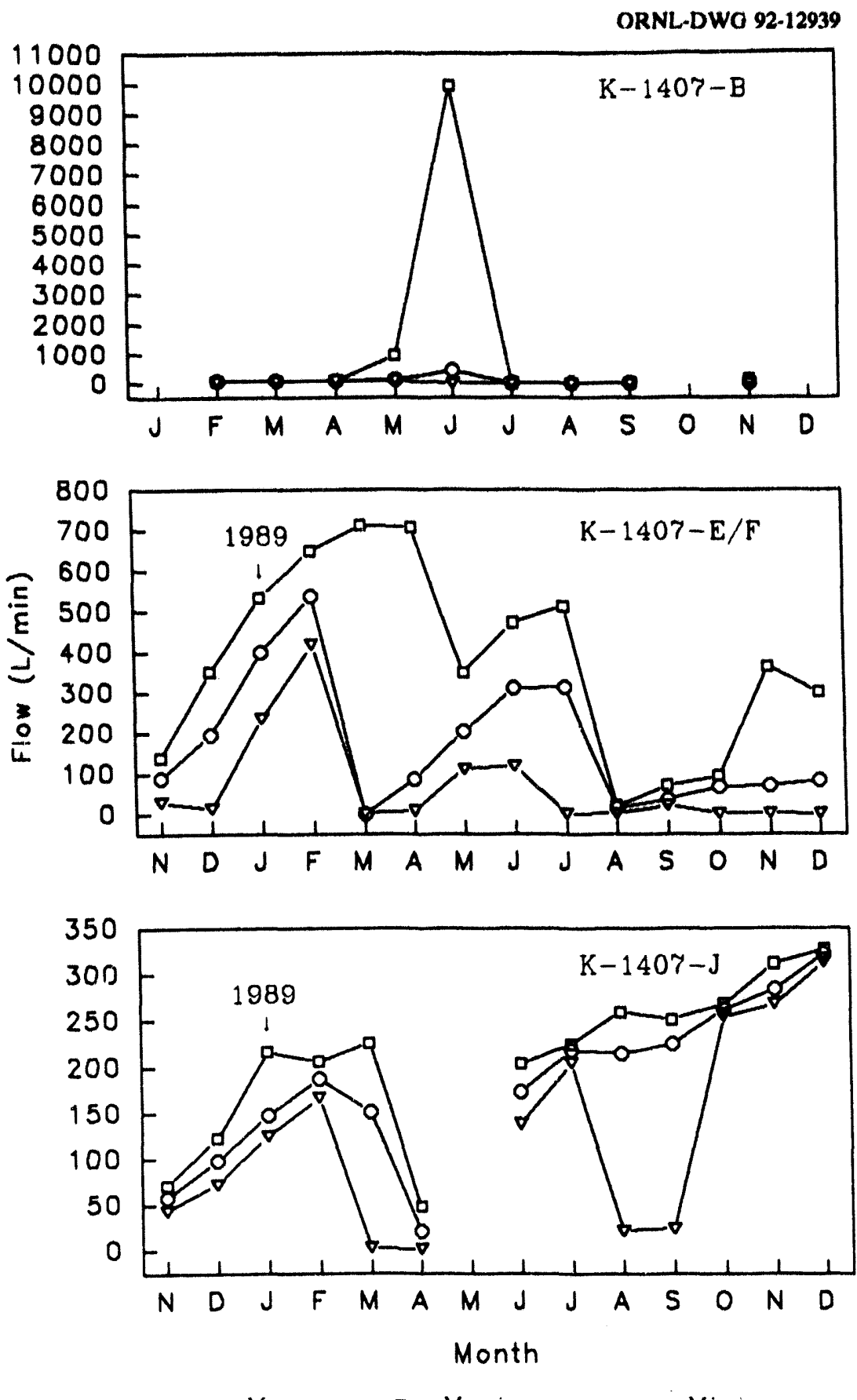

Fig. A.2. Mean monthly discharge from pond K-1407-B for 1988 and from K-1407-E/F and K-1407-J for November 1988-December 1989. Data were not available for K-1407-J from May to June 1989. Pond K-1407-B closed October 1988 and was replaced with K-1407-E/F (nonhazardous effluent) and K-1407-J (hazardous effluent). 
Appendix B

MEAN CONCENTRATIONS OF SELECTED PARAMETERS

IN MITCHELL BRANCH WATER OBTAINED BY THE

OAK RIDGE K-25 SITE PROCESS SUPPORT

DEPARTMENT IN CONJUNCTION WITH

THE TOXICITY TESTS CONDUCTED

DURING JULY 1988 THROUGH

DECEMBER 1989 
Tuble B.1. Moan (range in parentheses) concentrations of selected parameters (in milligrams per liter) in Mitchell Branch water obtained by the

Oat Ridee K-25 Site Proceses Support Department in conjunction with the taxicity test conducted

during Juhy 1988

\begin{tabular}{|c|c|c|c|}
\hline \multirow[b]{2}{*}{ Parameter } & \multicolumn{3}{|c|}{ Mitchell Branch kilometer } \\
\hline & 1.0 & 0.54 & 0.45 \\
\hline Aluminum & $\begin{array}{c}0.56 \\
(0.22-1.10)\end{array}$ & $\begin{array}{c}7.3 \\
(0.58-14)\end{array}$ & $\begin{array}{c}7.5 \\
(0.95-14)\end{array}$ \\
\hline Calcium & $\begin{array}{c}40 \\
(36-47)\end{array}$ & $\begin{array}{c}60 \\
(43-77)\end{array}$ & $\begin{array}{c}69 \\
(65-72)\end{array}$ \\
\hline Chloride & $\begin{array}{c}15 \\
(4-21)\end{array}$ & $\begin{array}{c}66 \\
(43-77)\end{array}$ & $\begin{array}{c}83 \\
(74-92)\end{array}$ \\
\hline Dissolved solids & $\begin{array}{c}259 \\
(222-306)\end{array}$ & $\begin{array}{c}387 \\
(286-488)\end{array}$ & $\begin{array}{c}471 \\
(450-492)\end{array}$ \\
\hline Fluoride & $\begin{array}{c}0.13 \\
(0.1-0.2)\end{array}$ & $\begin{array}{c}0.35 \\
(0.2-0.5)\end{array}$ & $\begin{array}{c}0.4 \\
(0.4-0.4)\end{array}$ \\
\hline Iron & $\begin{array}{c}0.79 \\
(0.4-1.4)\end{array}$ & $\begin{array}{c}3.6 \\
(1.6-5.6)\end{array}$ & $\begin{array}{c}4.7 \\
(3.1-6.2)\end{array}$ \\
\hline Magnesium & $\begin{array}{c}9.6 \\
(9.3-10.0)\end{array}$ & $\begin{array}{c}11.0 \\
(11-12)\end{array}$ & $\begin{array}{c}11.5 \\
(11-12)\end{array}$ \\
\hline Manganese & $\begin{array}{c}0.06 \\
(0.032-0.12)\end{array}$ & $\begin{array}{c}0.11 \\
(0.08-0.14)\end{array}$ & $\begin{array}{c}0.17 \\
(0.16-0.17)\end{array}$ \\
\hline Nickel & $\begin{array}{c}0.09 \\
(0.08-0.12)\end{array}$ & $\begin{array}{c}0.19 \\
(0.15-0.22)\end{array}$ & $\begin{array}{c}0.28 \\
(0.20-0.35)\end{array}$ \\
\hline Silicon & $\begin{array}{c}2.1 \\
(0.7-3.8)\end{array}$ & $\begin{array}{c}6.1 \\
(1.2-11)\end{array}$ & $\begin{array}{c}8.9 \\
(2.8-15)\end{array}$ \\
\hline Sodium & $\begin{array}{c}20 \\
(15-30)\end{array}$ & $\begin{array}{c}34 \\
(20-48)\end{array}$ & $\begin{array}{c}41 \\
(37-45)\end{array}$ \\
\hline Sulfate & $\begin{array}{c}33 \\
(8-46)\end{array}$ & $\begin{array}{c}108 \\
(65-151)\end{array}$ & $\begin{array}{c}141 \\
(118-163)\end{array}$ \\
\hline Suspended solids & $\begin{array}{c}13 \\
(3-30)\end{array}$ & $\begin{array}{c}143 \\
(6-280)\end{array}$ & $\begin{array}{c}138 \\
(13-262)\end{array}$ \\
\hline
\end{tabular}

Source: M. A. McGaha, 1989, Toxicity Monitoring at ORGDP July-September 1988, Report No. K/QT-288, Oak Ridge Gaseous Diffusion Plant, Oak Ridge, Tennessee.

Note: Two samples were taken from Mitchell Branch kilometer (MIK) 0.54 and 0.45 , and 3 were taken from MIK 1.0. 
Thble B.2. Mean (range in parentheses) concentrations of selocted parameters (in milligrams per Uiter) in Mitchell Branch water obtained by the Oak Ridge K-25 Site Process Support

Dopartment in conjunction with the toxicity test conducted during September 1988

\begin{tabular}{|c|c|c|c|c|c|}
\hline \multirow[b]{2}{*}{ Parameter } & \multicolumn{5}{|c|}{ Mitchell Branch kilometer } \\
\hline & 1.43 & 0.71 & 0.54 & 0.45 & 0.12 \\
\hline Aluminum & $\begin{array}{c}0.45 \\
(0.21-0.64)\end{array}$ & $\begin{array}{c}0.57 \\
(0.55-0.58)\end{array}$ & $\begin{array}{c}0.64 \\
(0.27-1.0)\end{array}$ & $\begin{array}{c}0.41 \\
(0.18-0.79)\end{array}$ & $\begin{array}{c}0.25 \\
(0.09-0.68)\end{array}$ \\
\hline Calcium & $\begin{array}{c}27 \\
(26-27)\end{array}$ & $\begin{array}{c}38 \\
(36-39)\end{array}$ & $\begin{array}{c}83 \\
(53-150)\end{array}$ & $\begin{array}{c}78 \\
(56-130)\end{array}$ & $\begin{array}{c}70 \\
(53-97)\end{array}$ \\
\hline Chloride & $\stackrel{1}{(1-1)}$ & $\begin{array}{c}25 \\
(24-26)\end{array}$ & $\begin{array}{c}106 \\
(56-218)\end{array}$ & $\begin{array}{c}78 \\
(37-168)\end{array}$ & $\begin{array}{c}58 \\
(28-119)\end{array}$ \\
\hline Dissolved solids & $\begin{array}{c}150 \\
(136-172)\end{array}$ & $\begin{array}{c}242 \\
(240-244)\end{array}$ & $\begin{array}{c}595 \\
(348-1116)\end{array}$ & $\begin{array}{c}514 \\
(324-922)\end{array}$ & $\begin{array}{c}463 \\
(342-664)\end{array}$ \\
\hline Fluoride & $\begin{array}{c}\mathrm{BD}^{\circ} \\
(<0.1)\end{array}$ & $\begin{array}{c}0.15 \\
(B D-0.2)\end{array}$ & $\begin{array}{c}0.89 \\
(0.4-2.0)\end{array}$ & $\begin{array}{c}0.73 \\
(0.4-1.6)\end{array}$ & $\begin{array}{c}0.66 \\
(0.4-1.1)\end{array}$ \\
\hline Iron & $\begin{array}{c}0.45 \\
(0.23-0.65)\end{array}$ & $\begin{array}{c}0.60 \\
(0.57-0.63)\end{array}$ & $\begin{array}{c}0.77 \\
(0.38-1.1)\end{array}$ & $\begin{array}{c}0.58 \\
(0.26-0.96)\end{array}$ & $\begin{array}{c}0.39 \\
(0.16-0.76)\end{array}$ \\
\hline Magnesium & $\begin{array}{c}13.7 \\
(13-14)\end{array}$ & $\begin{array}{c}7.3 \\
(9.3-9.3)\end{array}$ & $\begin{array}{c}11.7 \\
(9.9-15)\end{array}$ & $\begin{array}{c}12.3 \\
(11-15)\end{array}$ & $\begin{array}{c}11.7 \\
(10-13)\end{array}$ \\
\hline Manganese & $\begin{array}{c}0.05 \\
(0.03-0.07)\end{array}$ & $\begin{array}{c}0.08 \\
(0.06-0.10)\end{array}$ & $\begin{array}{c}0.11 \\
(0.06-0.14)\end{array}$ & $\begin{array}{c}0.12 \\
(0.08-0.16)\end{array}$ & $\begin{array}{c}0.14 \\
(0.03-0.23)\end{array}$ \\
\hline Nickel & $\begin{array}{c}\text { BD } \\
(<0.01)\end{array}$ & $\begin{array}{c}0.03 \\
(0.03-0.03)\end{array}$ & $\begin{array}{c}0.07 \\
(0.04-0.14)\end{array}$ & $\begin{array}{c}0.05 \\
(0.04-0.11)\end{array}$ & $\begin{array}{c}0.043 \\
(0.02-0.07)\end{array}$ \\
\hline Silicon & $\begin{array}{c}4.0 \\
(3.7-4.4)\end{array}$ & $\begin{array}{c}2.4 \\
(2.3-2.4)\end{array}$ & $\begin{array}{c}3.1 \\
(2.7-3.5)\end{array}$ & $\begin{array}{c}1.9 \\
(0.8-2.6)\end{array}$ & $\begin{array}{c}2.4 \\
(0.5-4.1)\end{array}$ \\
\hline Sodium & $\begin{array}{c}0.9 \\
(0.7-1.0)\end{array}$ & $\begin{array}{c}22 \\
(21-22)\end{array}$ & $\begin{array}{c}75 \\
(37-150)\end{array}$ & $\begin{array}{c}62 \\
(33-120)\end{array}$ & $\begin{array}{c}51 \\
(25-80)\end{array}$ \\
\hline Sulfate & $\begin{array}{c}2 \\
(2-2)\end{array}$ & $\begin{array}{c}48 \\
(47-49)\end{array}$ & $\begin{array}{c}187 \\
(93-414)\end{array}$ & $\begin{array}{c}174 \\
(97-333)\end{array}$ & $\begin{array}{c}143 \\
(75-239)\end{array}$ \\
\hline Suspended solids & $\begin{array}{c}28 \\
(6-60)\end{array}$ & $\begin{array}{c}9 \\
(6-11)\end{array}$ & $\begin{array}{c}16 \\
(5-22)\end{array}$ & $\begin{array}{c}8 \\
(2-13)\end{array}$ & $\begin{array}{c}2 \\
(1-6)\end{array}$ \\
\hline
\end{tabular}

${ }^{\mathrm{A}} \mathrm{BD}=$ below detection.

Source: M. A. McGaha, 1989, Toxicity Monitoring at ORGDP July-September 1988, Report No. K/QT-288, Oak Ridge Gaseous Diffusion Plant, Oak Ridge, Tennessee.

Note: Seven samples were taken at each locations. 
Table B.3. Mean (range in parentheces) concentrations of selocted parameters (in milligrams per liter) in Mitchell Branch water obtuined by the Oat Ridge K-25 Site Proceas Support Department in conjunction with the toricity test conducted during November 1988

\begin{tabular}{|c|c|c|c|c|c|c|}
\hline \multirow[b]{2}{*}{ Parameter } & \multicolumn{6}{|c|}{ Mitchell Branch kjlometer } \\
\hline & 1.43 & 1.00 & 0.71 & 0.54 & 0.45 & 0.12 \\
\hline Aluminum & $\begin{array}{c}1.01 \\
(0.15-2.8)\end{array}$ & $\begin{array}{c}0.11 \\
\left(\mathrm{BD}^{2}-0.35\right)\end{array}$ & $\begin{array}{c}0.89 \\
(B D-5.4)\end{array}$ & $\begin{array}{c}0.26 \\
(0.05-0.78)\end{array}$ & $\begin{array}{c}0.26 \\
(0.04-0.70)\end{array}$ & $\begin{array}{c}0.29 \\
(0.13-0.44)\end{array}$ \\
\hline Calcium & $\begin{array}{c}22 \\
(21-2 A)\end{array}$ & $\begin{array}{c}55 \\
(30-65)\end{array}$ & $\begin{array}{c}57 \\
(41-86)\end{array}$ & $\begin{array}{c}54 \\
(40-87)\end{array}$ & $\begin{array}{c}60 \\
(40-81)\end{array}$ & $\begin{array}{c}70 \\
(45-98)\end{array}$ \\
\hline Chloride & $\begin{array}{l}\text { BD } \\
(<10)\end{array}$ & $\begin{array}{c}11 \\
(B D-15)\end{array}$ & $\begin{array}{c}71 \\
(13-296)\end{array}$ & $\begin{array}{c}74 \\
(17-187)\end{array}$ & $\begin{array}{c}74 \\
(14-187)\end{array}$ & $\begin{array}{c}104 \\
(18-279)\end{array}$ \\
\hline Dissolved solids & $\begin{array}{c}121 \\
(102-146)\end{array}$ & $\begin{array}{c}253 \\
(200-274)\end{array}$ & $\begin{array}{c}639 \\
(220-1752)\end{array}$ & $\begin{array}{c}640 \\
(250-1636)\end{array}$ & $\begin{array}{c}547 \\
(238-1252)\end{array}$ & $\begin{array}{c}358 \\
(116-1620)\end{array}$ \\
\hline Fluoride & BD & $\begin{array}{c}0.20 \\
(0.1-0.3)\end{array}$ & $\begin{array}{c}2.2 \\
(0.2-9.8)\end{array}$ & $\begin{array}{c}2.1 \\
(0.2-9.0)\end{array}$ & $\begin{array}{c}1.5 \\
(0.2-6.2)\end{array}$ & $\begin{array}{c}2.2 \\
(0.3-8)\end{array}$ \\
\hline Iron & $\begin{array}{c}0.99 \\
(0.22-2.30)\end{array}$ & $\begin{array}{c}0.23 \\
(0.09-0.46)\end{array}$ & $\begin{array}{c}0.74 \\
(0.08-3.9)\end{array}$ & $\begin{array}{c}0.39 \\
(0.16-1.2)\end{array}$ & $\begin{array}{c}0.41 \\
(0.14-0.69)\end{array}$ & $\begin{array}{c}0.51 \\
(0.36-0.76)\end{array}$ \\
\hline Magnesium & $\begin{array}{c}13.1 \\
(12-14)\end{array}$ & $\begin{array}{c}15.6 \\
(9-18)\end{array}$ & $\begin{array}{c}12.0 \\
(11-14)\end{array}$ & $\begin{array}{c}13.0 \\
(11-15)\end{array}$ & $\begin{array}{c}12.6 \\
(11-14)\end{array}$ & $\begin{array}{c}13.3 \\
(12-16)\end{array}$ \\
\hline Manganese & $\begin{array}{c}0.09 \\
(0.05-0.16)\end{array}$ & $\begin{array}{c}0.05 \\
(0.03-0.15)\end{array}$ & $\begin{array}{c}0.07 \\
(0.04-0.12)\end{array}$ & $\begin{array}{c}0.12 \\
(0.09-0.20)\end{array}$ & $\begin{array}{c}0.16 \\
(0.12-0.25)\end{array}$ & $\begin{array}{c}0.24 \\
(0.18-0.35)\end{array}$ \\
\hline Nickel & $\begin{array}{c}\text { BD } \\
(<0.01)\end{array}$ & $\begin{array}{c}0.01 \\
(B D-0.01)\end{array}$ & $\begin{array}{c}0.03 \\
(\mathrm{BD}-0.12)\end{array}$ & $\begin{array}{c}0.03 \\
(B D-0.07)\end{array}$ & $\begin{array}{c}0.02 \\
(B D-0.03)\end{array}$ & $\begin{array}{c}0.02 \\
(B D-0.04)\end{array}$ \\
\hline Silicon & $\begin{array}{c}5.4 \\
(4.5-8.6)\end{array}$ & $\begin{array}{c}3.5 \\
(1.8-4.1)\end{array}$ & $\begin{array}{c}3.4 \\
(2-9.3)\end{array}$ & $\begin{array}{c}2.9 \\
(2.4-3.5)\end{array}$ & $\begin{array}{c}2.8 \\
(2.5-3.2)\end{array}$ & $\begin{array}{c}2.9 \\
(2.4-3.3)\end{array}$ \\
\hline Sodium & $\begin{array}{c}1 \\
(0.96-1.8)\end{array}$ & $\begin{array}{c}10 \\
(6.5-23)\end{array}$ & $\begin{array}{c}148 \\
(17-540)\end{array}$ & $\begin{array}{c}140 \\
(20-490)\end{array}$ & $\begin{array}{c}105 \\
(16-340)\end{array}$ & $\begin{array}{c}136 \\
(20-420)\end{array}$ \\
\hline Sulfate & $\begin{array}{l}\text { BD } \\
(<10)\end{array}$ & $\begin{array}{c}56 \\
(51-60)\end{array}$ & $\begin{array}{c}134 \\
(26-468)\end{array}$ & $\begin{array}{c}169 \\
(51-412)\end{array}$ & $\begin{array}{c}142 \\
(49-309)\end{array}$ & $\begin{array}{c}184 \\
(48-404)\end{array}$ \\
\hline Suspended solids & $\begin{array}{c}37 \\
(5-115)\end{array}$ & $\begin{array}{c}3 \\
(B D-6)\end{array}$ & $\begin{array}{c}18 \\
\text { (BD-96) }\end{array}$ & $\begin{array}{c}5 \\
(B D-16)\end{array}$ & $\begin{array}{c}5 \\
(1-15)\end{array}$ & $\begin{array}{c}5 \\
(3-9)\end{array}$ \\
\hline
\end{tabular}

$\mathrm{BD}=$ below detection.

Source: M. A. McGaha, 1989, Toxicity Monitoring at ORGDP October-December 1988, Report No. K/QT-0311, Oak Ridge Gaseous Diffusion Plant, Oak Ridge, Tennessee.

Note: Seven samples were taken at each location. 
Table B.4. Mean (range in parentheses) concentrations of selected parameters (in milligrams per liter) in Mitchell Branch water obtained by the Oak Ridge K-25 Site Process Support Department in conjunction with the toxicity test conducted during January 1989

\begin{tabular}{|c|c|c|c|c|c|c|}
\hline \multirow[b]{2}{*}{ Parameter } & \multicolumn{6}{|c|}{ Mitchell Branch kilometer } \\
\hline & 1.43 & 1.00 & 0.71 & 0.54 & 0.45 & 0.12 \\
\hline Aluminum & $\begin{array}{c}0.36 \\
(0.22-0.58)\end{array}$ & $\begin{array}{c}0.41 \\
(0.29-0.65)\end{array}$ & $\begin{array}{c}0.32 \\
(0.08-0.54)\end{array}$ & $\begin{array}{c}0.30 \\
(0.16-0.52)\end{array}$ & $\begin{array}{c}0.22 \\
(0.11-0.38)\end{array}$ & $\begin{array}{c}0.20 \\
(0.05-0.35)\end{array}$ \\
\hline Calcium & $\begin{array}{c}13 \\
(11-14)\end{array}$ & $\begin{array}{c}25 \\
(23-27)\end{array}$ & $\begin{array}{c}38 \\
(33-62)\end{array}$ & $\begin{array}{c}44 \\
(37-64)\end{array}$ & $\begin{array}{c}46 \\
(39-65)\end{array}$ & $\begin{array}{c}52 \\
(43-70)\end{array}$ \\
\hline Chloride & $\begin{array}{c}4 \\
\left(B D^{2}-10\right)\end{array}$ & $\begin{array}{c}2 \\
(2-2)\end{array}$ & $\begin{array}{c}19 \\
(8-79)\end{array}$ & $\begin{array}{c}25 \\
(10-74)\end{array}$ & $\begin{array}{c}24 \\
(10-72)\end{array}$ & $\begin{array}{c}27 \\
(11-78)\end{array}$ \\
\hline Dissolved solids & $\begin{array}{c}63 \\
(56-74)\end{array}$ & $\begin{array}{c}125 \\
(112-142)\end{array}$ & $\begin{array}{c}229 \\
(190-432)\end{array}$ & $\begin{array}{c}251 \\
(190-410)\end{array}$ & $\begin{array}{c}254 \\
(204-408)\end{array}$ & $\begin{array}{c}280 \\
(214-470)\end{array}$ \\
\hline Fluoride & $\begin{array}{c}\text { BD } \\
(<0.1)\end{array}$ & BD & $\begin{array}{c}0.14 \\
(B D-0.40)\end{array}$ & $\begin{array}{c}0.10 \\
(0.1-0.1)\end{array}$ & $\begin{array}{c}0.11 \\
(0.1-0.2)\end{array}$ & $\begin{array}{c}0.20 \\
(0.2-0.2)\end{array}$ \\
\hline Iron & $\begin{array}{c}0.38 \\
(0.26-0.62)\end{array}$ & $\begin{array}{c}0.41 \\
(0.34-0.52)\end{array}$ & $\begin{array}{c}0.32 \\
(0.15-0.49)\end{array}$ & $\begin{array}{c}0.35 \\
(0.2-0.55)\end{array}$ & $\begin{array}{c}0.45 \\
(0.32-0.63)\end{array}$ & $\begin{array}{c}0.50 \\
(0.35-0.68)\end{array}$ \\
\hline Magnesium & $\begin{array}{c}7.0 \\
(5.9-8)\end{array}$ & $\begin{array}{c}9.9 \\
(8.6-11)\end{array}$ & $\begin{array}{c}10.0 \\
(9.3-12)\end{array}$ & $\begin{array}{c}10.4 \\
(9.7-12)\end{array}$ & $\begin{array}{c}10.9 \\
(10-12)\end{array}$ & $\begin{array}{c}11.7 \\
(11-13)\end{array}$ \\
\hline Manganese & $\begin{array}{c}0.05 \\
(0.05-0.07)\end{array}$ & $\begin{array}{c}0.06 \\
(0.04-0.11)\end{array}$ & $\begin{array}{c}0.07 \\
0.06-0.08)\end{array}$ & $\begin{array}{c}0.19 \\
(0.16-0.24)\end{array}$ & $\begin{array}{c}0.25 \\
(0.20-0.29)\end{array}$ & $\begin{array}{c}0.28 \\
(0.22-0.34)\end{array}$ \\
\hline Nickel & $\underset{(<0.01)}{\text { BD }}$ & $\begin{array}{c}0.01 \\
(\mathrm{BD}-0.01)\end{array}$ & $\begin{array}{c}0.05 \\
(0.02-0.23)\end{array}$ & $\begin{array}{c}0.05 \\
(0.01-0.22)\end{array}$ & $\begin{array}{c}0.04 \\
(B D-0.19)\end{array}$ & $\begin{array}{c}0.05 \\
(0.01-0.22)\end{array}$ \\
\hline Silicon & $\begin{array}{c}3.8 \\
(3.6-4.1)\end{array}$ & $\begin{array}{c}3.9 \\
(3.7-4.4)\end{array}$ & $\begin{array}{c}3.5 \\
(2.8-4.2)\end{array}$ & $\begin{array}{c}3.5 \\
(3.2-3.8)\end{array}$ & $\begin{array}{c}3.4 \\
(3.0-4.0)\end{array}$ & $\begin{array}{c}3.4 \\
(3.0-3.6)\end{array}$ \\
\hline Sodium & $\begin{array}{c}1 \\
(1.0-1.2)\end{array}$ & $\begin{array}{c}2 \\
(1.9-2.4)\end{array}$ & $\begin{array}{c}17 \\
(9.7-54)\end{array}$ & $\begin{array}{c}21 \\
(11-53)\end{array}$ & $\begin{array}{c}20 \\
(10-52)\end{array}$ & $\begin{array}{c}23 \\
(10-63)\end{array}$ \\
\hline Sulfate & $\begin{array}{c}6 \\
(B D-10)\end{array}$ & $\begin{array}{c}14 \\
(13-14)\end{array}$ & $\begin{array}{c}44 \\
(32-106)\end{array}$ & $\begin{array}{c}51 \\
(34-100)\end{array}$ & $\begin{array}{c}54 \\
(36-102)\end{array}$ & $\begin{array}{c}21 \\
(6-103)\end{array}$ \\
\hline Suspended solids & $\begin{array}{c}4.3 \\
(3-7)\end{array}$ & $\begin{array}{c}3.1 \\
(2-5)\end{array}$ & $\begin{array}{c}3.1 \\
(1-5)\end{array}$ & $\begin{array}{c}4.6 \\
(2-7)\end{array}$ & $\begin{array}{c}3.6 \\
(2-6)\end{array}$ & $\begin{array}{c}3.3 \\
(1-5)\end{array}$ \\
\hline
\end{tabular}

${ }^{a} \mathrm{BD}=$ below detection.

Source: M. A. McGaha, 1989, Toxicity howitoring at ORGDP January-March 1989, Report No. K/QT-312, Oak Ridge Gaseous Diffusion Fiant, Oak Ridge, Tennessee.

Note: Seven samples were taken from each location. 
Table B.5. Mean (range in parentheses) concentrations of selected parameters (in milligrams per liter) in Mitchell Branch water obtained by the Oak Ridge K-25 Site Process Support Department in conjunction with the toxicity test conducted during March 1989

\begin{tabular}{|c|c|c|c|c|c|c|}
\hline \multirow[b]{2}{*}{ Parameter } & \multicolumn{6}{|c|}{ Mitchell Branch kilometer } \\
\hline & 1.43 & 1.00 & 0.71 & 0.54 & 0.45 & 0.12 \\
\hline Aluminum & $\begin{array}{l}\mathrm{BD}^{n} \\
(<0.5)\end{array}$ & $\begin{array}{c}0.60 \\
(B D-1.2)\end{array}$ & $\begin{array}{c}0.53 \\
(B D-0.74)\end{array}$ & $\begin{array}{c}0.58 \\
(B D-0.88)\end{array}$ & $\begin{array}{c}0.70 \\
\text { (BD-1.2) }\end{array}$ & $\begin{array}{c}0.80 \\
(0.53-0.92)\end{array}$ \\
\hline Calcium & $\begin{array}{c}13 \\
(12-15)\end{array}$ & $\begin{array}{c}26 \\
(23-28)\end{array}$ & $\begin{array}{c}43 \\
(31-70)\end{array}$ & $\begin{array}{c}49 \\
(37-76)\end{array}$ & $\begin{array}{c}52 \\
(43-75)\end{array}$ & $\begin{array}{c}55 \\
(47-80)\end{array}$ \\
\hline Chloride & $\begin{array}{c}\text { BD } \\
(<10)\end{array}$ & BD & $\begin{array}{c}28 \\
\text { (BD-91) }\end{array}$ & $\begin{array}{c}31 \\
(B D-96)\end{array}$ & $\begin{array}{c}27 \\
(\mathrm{BD}-82)\end{array}$ & $\begin{array}{c}35 \\
(15-92)\end{array}$ \\
\hline Dissolved solids & $\begin{array}{c}76 \\
(66-84)\end{array}$ & $\begin{array}{c}110 \\
(102-118)\end{array}$ & $\begin{array}{c}231 \\
(146-412)\end{array}$ & $\begin{array}{c}269 \\
(186-482)\end{array}$ & $\begin{array}{c}275 \\
(206-472)\end{array}$ & $\begin{array}{c}299 \\
(215-524)\end{array}$ \\
\hline Fluoride & $\begin{array}{c}\text { BD } \\
(<0.1)\end{array}$ & $\begin{array}{c}0.11 \\
\text { (BD-0.2) }\end{array}$ & $\begin{array}{c}0.11 \\
(B D-0.20)\end{array}$ & $\begin{array}{c}0.11 \\
(0.1-0.2)\end{array}$ & $\begin{array}{c}0.11 \\
(B D-0.2)\end{array}$ & $\begin{array}{c}0.23 \\
(0.2-0.4)\end{array}$ \\
\hline Iron & $\begin{array}{c}0.22 \\
\text { (BD-0.48) }\end{array}$ & $\begin{array}{c}0.11 \\
(B D-0.2)\end{array}$ & $\begin{array}{c}0.48 \\
(0.24-1.2)\end{array}$ & $\begin{array}{c}0.44 \\
(0.33-0.58)\end{array}$ & $\begin{array}{c}0.44 \\
(0.33-0.57)\end{array}$ & $\begin{array}{c}0.53 \\
(0.4-0.6)\end{array}$ \\
\hline Magnesium & $\begin{array}{c}6.9 \\
(6-7.6)\end{array}$ & $\begin{array}{c}9.3 \\
8.2-10)\end{array}$ & $\begin{array}{c}10.5 \\
(8.6-14)\end{array}$ & $\begin{array}{c}11.2 \\
(9.4-15)\end{array}$ & $\begin{array}{c}11.9 \\
(11-15)\end{array}$ & $\begin{array}{c}12.9 \\
(12-16)\end{array}$ \\
\hline Manganese & $\begin{array}{c}0.05 \\
(B D-0.15)\end{array}$ & $\begin{array}{c}0.04 \\
(B D-0.06)\end{array}$ & $\begin{array}{c}0.14 \\
(0.08-0.20)\end{array}$ & $\begin{array}{c}0.21 \\
(0.18-0.25)\end{array}$ & $\begin{array}{c}0.31 \\
(0.19-0.39)\end{array}$ & $\begin{array}{c}0.27 \\
(0.22-0.35)\end{array}$ \\
\hline Nickel & $\begin{array}{c}\text { BD } \\
(<0.25)\end{array}$ & BD & $\begin{array}{c}0.25 \\
\text { BD-0.28 }\end{array}$ & BD & BD & BD \\
\hline Silicon & $\begin{array}{c}5.3 \\
(4.4-8.4)\end{array}$ & $\begin{array}{c}5.1 \\
(4.6-6.2)\end{array}$ & $\begin{array}{c}5.0 \\
(4.2-6.8)\end{array}$ & $\begin{array}{c}4.7 \\
(4.2-5.5)\end{array}$ & $\begin{array}{c}5.2 \\
(4.2-6.7)\end{array}$ & $\begin{array}{c}4.2 \\
(3.4-5.7)\end{array}$ \\
\hline Sodium & $\begin{array}{c}4 \\
(1.8-8.7)\end{array}$ & $\begin{array}{c}5 \\
(4.6-6.2)\end{array}$ & $\begin{array}{c}27 \\
(9.9-81)\end{array}$ & $\begin{array}{c}31 \\
(13-78)\end{array}$ & $\begin{array}{c}32 \\
(14-69)\end{array}$ & $\begin{array}{c}31 \\
(14-73)\end{array}$ \\
\hline Sulfate & $\begin{array}{l}\text { BD } \\
(<10)\end{array}$ & $\begin{array}{c}11 \\
(10-12)\end{array}$ & $\begin{array}{c}49 \\
(22-136)\end{array}$ & $\begin{array}{c}48 \\
(\mathrm{BD}-116)\end{array}$ & $\begin{array}{c}52 \\
(32-114)\end{array}$ & $\begin{array}{c}56 \\
(32-124)\end{array}$ \\
\hline Suspended solids & $\begin{array}{c}6.4 \\
(3-11)\end{array}$ & $\begin{array}{c}4.1 \\
(2-7)\end{array}$ & $\begin{array}{c}12.7 \\
(\mathrm{BD}-57)\end{array}$ & $\begin{array}{c}9.7 \\
(5-15)\end{array}$ & $\begin{array}{c}6.6 \\
(3-9)\end{array}$ & $\begin{array}{c}4.0 \\
(2-6)\end{array}$ \\
\hline
\end{tabular}

${ }^{\mathrm{B}} \mathrm{BD}=$ below detection.

Source: M. A. McGaha, 1989, Toxicity Monitoring at ORGDP January-March 1989, Report No. K/QT-312, Oak Ridge Gaseous Diffusion Plant, Oak Ridge, Tennessee.

Note: Seven samples were taken from each location. 
Tuble B.6. Mean (range in parentheses) concentrations of selected parameters (in milligrams per liter) in Mitchell Branch water obtained by the Oak Ridge K-25 Site Process Support Department in conjunction with the toxicity test conducted during May 1989

\begin{tabular}{|c|c|c|c|c|c|c|}
\hline \multirow[b]{2}{*}{ Parameter } & \multicolumn{6}{|c|}{ Mitchell Branch kilometer } \\
\hline & 1.43 & 1.00 & 0.71 & 0.54 & 0.45 & 0.12 \\
\hline Aluminum & $\begin{array}{c}0.86 \\
(0.32-3.0)\end{array}$ & $\begin{array}{c}0.91 \\
(0.44-3.0)\end{array}$ & $\begin{array}{c}0.42 \\
(0.1-2.0)\end{array}$ & $\begin{array}{c}0.75 \\
(0.03-4.0)\end{array}$ & $\begin{array}{c}0.98 \\
(0.11-4.9)\end{array}$ & $\begin{array}{c}1.05 \\
(0.27-5.4)\end{array}$ \\
\hline Calcium & $\begin{array}{c}17 \\
(15-17)\end{array}$ & $\begin{array}{c}29 \\
(21-30)\end{array}$ & $\begin{array}{c}39 \\
(20-78)\end{array}$ & $\begin{array}{c}43 \\
(20-83)\end{array}$ & $\begin{array}{c}48 \\
(20-89)\end{array}$ & $\begin{array}{c}54 \\
(17-79)\end{array}$ \\
\hline Chloride & $\begin{array}{c}2 \\
(1-2)\end{array}$ & $\begin{array}{c}3 \\
(2-3)\end{array}$ & $\begin{array}{c}34 \\
(3-113)\end{array}$ & $\begin{array}{c}34 \\
(3-115)\end{array}$ & $\begin{array}{c}30 \\
(8-87)\end{array}$ & $\begin{array}{c}43 \\
(6-88)\end{array}$ \\
\hline Dissolved solids & $\begin{array}{c}91 \\
(86-94)\end{array}$ & $\begin{array}{c}141 \\
(108-152)\end{array}$ & $\begin{array}{c}234 \\
(102-476)\end{array}$ & $\begin{array}{c}273 \\
(138-550)\end{array}$ & $\begin{array}{c}263 \\
(98-514)\end{array}$ & $\begin{array}{c}227 \\
(84-378)\end{array}$ \\
\hline Fluoride & $\begin{array}{c}\mathrm{BD}^{\mathbf{a}} \\
(<0.1)\end{array}$ & BD & BD & $\begin{array}{c}0.13 \\
(0.1-0.2)\end{array}$ & $\begin{array}{c}0.16 \\
(0.1-0.2)\end{array}$ & $\begin{array}{c}0.36 \\
(0.2-1.3)\end{array}$ \\
\hline Iron & $\begin{array}{c}1.00 \\
(0.46-3.3)\end{array}$ & $\begin{array}{c}1.11 \\
(0.59-2.9)\end{array}$ & $\begin{array}{c}0.58 \\
(0.19-2.6)\end{array}$ & $\begin{array}{c}0.96 \\
(0.2-4.4)\end{array}$ & $\begin{array}{c}0.34 \\
(0.24-5.7)\end{array}$ & $\begin{array}{c}1.26 \\
(0.53-5.4)\end{array}$ \\
\hline Magnesium & $\begin{array}{c}8.8 \\
(5.2-9.9)\end{array}$ & $\begin{array}{c}10.9 \\
(5.5-13)\end{array}$ & $\begin{array}{c}8.9 \\
(4.5-12)\end{array}$ & $\begin{array}{c}9.3 \\
(4.5-13)\end{array}$ & $\begin{array}{c}9.4 \\
(3.7-13)\end{array}$ & $\begin{array}{c}9.7 \\
(3.3-12)\end{array}$ \\
\hline Manganese & $\begin{array}{c}0.16 \\
(0.07-0.36)\end{array}$ & $\begin{array}{c}0.20 \\
(0.14-0.3)\end{array}$ & $\begin{array}{c}0.06 \\
(0.05-0.11)\end{array}$ & $\begin{array}{c}0.10 \\
(0.09-0.12)\end{array}$ & $\begin{array}{c}0.17 \\
0.1-0.25)\end{array}$ & $\begin{array}{c}0.21 \\
(0.12-0.25)\end{array}$ \\
\hline Nickel & $\begin{array}{c}\mathrm{BD} \\
(<0.25)\end{array}$ & BD & $\begin{array}{c}0.01 \\
(\mathrm{BD}-0.018)\end{array}$ & $\begin{array}{c}0.01 \\
(B D-0.021)\end{array}$ & $\begin{array}{c}0.02 \\
(\mathrm{BD}-0.025)\end{array}$ & $\begin{array}{c}0.01 \\
(\mathrm{BD}-0.017)\end{array}$ \\
\hline Silicon & $\begin{array}{c}4.8 \\
(4.3-6.6)\end{array}$ & $\begin{array}{c}5.0 \\
(4.5-6.7)\end{array}$ & $\begin{array}{c}3.1 \\
(2.6-4.5)\end{array}$ & $\begin{array}{c}3.7 \\
(2.7-7.5)\end{array}$ & $\begin{array}{c}4.0 \\
(2.7-8.3)\end{array}$ & $\begin{array}{c}4.2 \\
(3.1-9.2)\end{array}$ \\
\hline Sodium & $\begin{array}{c}1 \\
(0.9-2.1)\end{array}$ & $(1.7-2.8)$ & $\begin{array}{c}22 \\
(2.7-50)\end{array}$ & $\begin{array}{c}22 \\
(2.4-51)\end{array}$ & $\begin{array}{c}22 \\
(5.2-49)\end{array}$ & $\begin{array}{c}31.7 \\
(4-553)\end{array}$ \\
\hline Sulfate & $\begin{array}{c}3 \\
(3-5)\end{array}$ & $\begin{array}{c}14 \\
(9-17)\end{array}$ & $\begin{array}{c}64 \\
(12-213)\end{array}$ & $\begin{array}{c}67 \\
(15-217)\end{array}$ & $\begin{array}{c}70 \\
(20-195)\end{array}$ & $\begin{array}{c}78 \\
(18-152)\end{array}$ \\
\hline Suspended solids & $\begin{array}{c}16.3 \\
(3-74)\end{array}$ & $\begin{array}{c}14.9 \\
(4-48)\end{array}$ & $\begin{array}{c}10.1 \\
(B D-46)\end{array}$ & $\begin{array}{c}12.1 \\
(B D-69)\end{array}$ & $\begin{array}{c}20.9 \\
(\mathrm{BD}-88)\end{array}$ & $\begin{array}{c}15.4 \\
(1-86)\end{array}$ \\
\hline
\end{tabular}

${ }^{\circ} \mathrm{BD}=$ below detection.

Source: M. A. McGaha, 1989, Taxicity Monitoring at ORGDP April-June 1989, Report No. K/QT-313, Oak Ridge Gaseous Diffusion Plant, Oak Ridge, Tennessee.

Note: Seven samples were taken from each location. 
Table B.7. Mean (range in parentheses) concentrations of selected parameters (in milligrams per liter) in Mitchell Branch water obtained by the Oak Ridge K-25 Site Process Support Department in conjunction with the toxicity test conducted during July 1989

\begin{tabular}{|c|c|c|c|c|c|c|}
\hline \multirow[b]{2}{*}{ Parameter } & \multicolumn{6}{|c|}{ Mitchell Branch kilometer } \\
\hline & 1.43 & 1.00 & 0.71 & 0.54 & 0.45 & 0.12 \\
\hline Aluminum & $\mathbf{N M}^{a}$ & $\begin{array}{c}0.50 \\
(0.15-0.85)\end{array}$ & $\begin{array}{c}0.10 \\
(0.07-0.18)\end{array}$ & $\begin{array}{c}0.29 \\
(0.08-1.3)\end{array}$ & $\begin{array}{c}0.36 \\
(0.11-0.36)\end{array}$ & $\begin{array}{c}0.22 \\
(0.10-0.4)\end{array}$ \\
\hline Calcium & $\begin{array}{c}19 \\
(19-20)\end{array}$ & $\begin{array}{c}42 \\
(34-79)\end{array}$ & $\begin{array}{c}61 \\
(44-79)\end{array}$ & $\begin{array}{c}58 \\
(43-73)\end{array}$ & $\begin{array}{c}64 \\
(55-75)\end{array}$ & $\begin{array}{c}67 \\
(56-87)\end{array}$ \\
\hline Chloride & $\begin{array}{c}1 \\
(1-1)\end{array}$ & $\begin{array}{c}8 \\
(2-45)\end{array}$ & $\begin{array}{c}35 \\
(15-57)\end{array}$ & $\begin{array}{c}32 \\
(21-56)\end{array}$ & $\begin{array}{c}32 \\
(23-44)\end{array}$ & $\begin{array}{c}40 \\
(20-92)\end{array}$ \\
\hline Dissolved solids & $\begin{array}{c}117 \\
(96-136\end{array}$ & $\begin{array}{c}209 \\
(158-450)\end{array}$ & $\begin{array}{c}336 \\
(246-460)\end{array}$ & $\begin{array}{c}366 \\
(250-660)\end{array}$ & $\begin{array}{c}348 \\
(246-580)\end{array}$ & $\begin{array}{c}349 \\
(242-610)\end{array}$ \\
\hline Fluoride & $\begin{array}{c}\mathrm{BD}^{b} \\
(<0.1)\end{array}$ & $\begin{array}{c}0.13 \\
(\mathrm{BD}-0.3)\end{array}$ & $\begin{array}{c}0.27 \\
(0.2-0.041)\end{array}$ & $\begin{array}{c}0.13 \\
(0.1-0.2)\end{array}$ & $\begin{array}{c}0.19 \\
(0.1-0.3)\end{array}$ & $\begin{array}{c}0.21 \\
(0.2-0.3)\end{array}$ \\
\hline Iron & $\begin{array}{c}0.49 \\
(0.41-0.61)\end{array}$ & $\begin{array}{c}0.99 \\
(0.51-1.7)\end{array}$ & $\begin{array}{c}0.17 \\
(0.1-0.5)\end{array}$ & $\begin{array}{c}0.54 \\
(0.18-2.4)\end{array}$ & $\begin{array}{c}0.80 \\
(0.35-2.2)\end{array}$ & $\begin{array}{c}0.57 \\
(0.21-0.88)\end{array}$ \\
\hline Magnesium & $\begin{array}{c}11.1 \\
(11-12)\end{array}$ & $\begin{array}{c}14.1 \\
(13-15)\end{array}$ & $\begin{array}{c}12.4 \\
(11-14)\end{array}$ & $\begin{array}{c}11.9 \\
(11-13)\end{array}$ & $\begin{array}{c}13 \\
(12-13)\end{array}$ & $\begin{array}{c}12.7 \\
(12-132)\end{array}$ \\
\hline Manganese & $\begin{array}{c}0.10 \\
(0.07-0.13)\end{array}$ & $\begin{array}{c}0.30 \\
(0.18-0.61)\end{array}$ & $\begin{array}{c}0.13 \\
0.1-0.19 \text { ) }\end{array}$ & $\begin{array}{c}0.19 \\
(0.10-0.41)\end{array}$ & $\begin{array}{c}0.28 \\
(0.24-0.28)\end{array}$ & $\begin{array}{c}0.30 \\
(0.11-0.35)\end{array}$ \\
\hline Nickel & $\begin{array}{c}0.01 \\
(B D-0.02)\end{array}$ & $\begin{array}{c}0.01 \\
(B D-0.02)\end{array}$ & $\begin{array}{c}0.02 \\
(0.02-0.04)\end{array}$ & $\begin{array}{c}0.02 \\
(0.01-0.05)\end{array}$ & $\begin{array}{c}0.02 \\
(0.02-0.03)\end{array}$ & $\begin{array}{c}0.02 \\
(0.02-0.03)\end{array}$ \\
\hline Silicon & $\begin{array}{c}4.1 \\
(3.9-4.3)\end{array}$ & $\begin{array}{c}4.6 \\
(3.6-5.2)\end{array}$ & $\begin{array}{c}3.6 \\
(3.5-3.7)\end{array}$ & $\begin{array}{c}3.8 \\
(3.3-5.3)\end{array}$ & $\begin{array}{c}3.8 \\
(3.5-4.6)\end{array}$ & $\begin{array}{c}3.5 \\
(3.4-3.8)\end{array}$ \\
\hline Sodium & $\begin{array}{c}0.8 \\
(0.7-0.8)\end{array}$ & $\begin{array}{c}8 \\
(12-44)\end{array}$ & $\begin{array}{c}31 \\
(16-53)\end{array}$ & $\begin{array}{c}35 \\
(15-110)\end{array}$ & $\begin{array}{c}32 \\
(13-87)\end{array}$ & $\begin{array}{c}40 \\
(46-228)\end{array}$ \\
\hline Sulfate & $\begin{array}{c}2 \\
(2-2)\end{array}$ & $\begin{array}{c}30 \\
(13-131)\end{array}$ & $\begin{array}{c}92 \\
(43-166)\end{array}$ & $\begin{array}{c}105 \\
(48-246)\end{array}$ & $\begin{array}{c}101 \\
(48-246)\end{array}$ & $\begin{array}{c}126 \\
(46-228)\end{array}$ \\
\hline Suspended solids & $\begin{array}{c}13 \\
(3-41)\end{array}$ & $\begin{array}{c}14 \\
(2-33)\end{array}$ & $\begin{array}{c}2 \\
\text { (BD-4) }\end{array}$ & $\begin{array}{c}15 \\
(B D-96)\end{array}$ & $\begin{array}{c}12 \\
(1-45)\end{array}$ & $\begin{array}{c}5 \\
(1-8)\end{array}$ \\
\hline
\end{tabular}

${ }^{a} \mathrm{NM}=$ not measured.

${ }^{b} \mathrm{BD}=$ below detection.

Source: M. A. McGaha, 1989, Toxicity Monitoring at ORGDP July-September 1988, Report

No. K/QT-342, Oak Ridge Gaseous Diffusion Plant, Oak Ridge, Tennessee.

Note: Seven samples were taken from each location. 
Table B.8. Mean (range in parentheses) concentrations of selected parameters (in milligrams per liter) in Mitchell Branch water obtained by the Oak Ridge K-25 Site Process Support Department in conjunction with the toxicity test conducted during September 1989

\begin{tabular}{|c|c|c|c|c|c|c|}
\hline \multirow[b]{2}{*}{ Parameter } & \multicolumn{6}{|c|}{ Mitchell Branch kilometer } \\
\hline & 1.43 & 1.00 & 0.71 & 0.54 & 0.45 & 0.12 \\
\hline Aluminum & $\begin{array}{c}0.95 \\
(0.2-1.9)\end{array}$ & $\begin{array}{c}1.01 \\
(0.47-1.6)\end{array}$ & $\begin{array}{c}1.32 \\
(0.22-2.9)\end{array}$ & $\begin{array}{c}1.90 \\
(0.11-8.1)\end{array}$ & $\begin{array}{c}1.44 \\
(0.08-6.2)\end{array}$ & $\begin{array}{c}3.5 \\
(0.16-14)\end{array}$ \\
\hline Calcium & $\begin{array}{c}20 \\
(17-22)\end{array}$ & $\begin{array}{c}35 \\
(30-41)\end{array}$ & $\begin{array}{c}50 \\
(12-94)\end{array}$ & $\begin{array}{c}56 \\
(30-85)\end{array}$ & $\begin{array}{c}56 \\
(28-77)\end{array}$ & $\begin{array}{c}53 \\
(24-74)\end{array}$ \\
\hline Chloride & $\begin{array}{c}2 \\
(1-2)\end{array}$ & $\begin{array}{c}2 \\
(2-3)\end{array}$ & $\begin{array}{c}30 \\
(4-116)\end{array}$ & $\begin{array}{c}32 \\
(10-96)\end{array}$ & $\begin{array}{c}27 \\
(7-73)\end{array}$ & $\begin{array}{c}16 \\
(6-32)\end{array}$ \\
\hline Dissolved solids & $\begin{array}{c}121 \\
(104-134)\end{array}$ & $\begin{array}{c}176 \\
(138-204)\end{array}$ & $\begin{array}{c}262 \\
(110-524)\end{array}$ & $\begin{array}{c}277 \\
(150-480)\end{array}$ & $\begin{array}{c}301 \\
(140-418)\end{array}$ & $\begin{array}{c}250 \\
(116-362)\end{array}$ \\
\hline Fluoride & $\begin{array}{c}\mathrm{BD}^{a} \\
(<0.1)\end{array}$ & $\begin{array}{c}0.12 \\
(\mathrm{BD}-0.2)\end{array}$ & $\begin{array}{c}0.16 \\
(\mathrm{BD}-0.53)\end{array}$ & $\begin{array}{c}0.19 \\
(\mathrm{BD}-0.3)\end{array}$ & $\begin{array}{c}0.20 \\
(0.1-0.3)\end{array}$ & $\begin{array}{c}0.24 \\
(0.2-0.3)\end{array}$ \\
\hline Iron & $\begin{array}{c}1.1 \\
(0.4-2.2\end{array}$ & $\begin{array}{c}1.10 \\
(0.7-1.7)\end{array}$ & $\begin{array}{c}0.16 \\
(0.28-4.1)\end{array}$ & $\begin{array}{c}2.24 \\
(0.14-9.6)\end{array}$ & $\begin{array}{c}2.01 \\
(0.3-8.3)\end{array}$ & $\begin{array}{c}4.07 \\
(0.45-16)\end{array}$ \\
\hline Magnesium & $\begin{array}{c}10.0 \\
(8.5-13)\end{array}$ & $\begin{array}{c}11.4 \\
(6.3-17)\end{array}$ & $\begin{array}{c}10.2 \\
(4.7-12)\end{array}$ & $\begin{array}{c}10.6 \\
(6.3-12)\end{array}$ & $\begin{array}{c}10.3 \\
(5.3-12)\end{array}$ & $\begin{array}{c}10.4 \\
(5.5-13)\end{array}$ \\
\hline Manganese & $\begin{array}{c}0.12 \\
(0.07-0.17)\end{array}$ & $\begin{array}{c}0.14 \\
(0.07-0.33)\end{array}$ & $\begin{array}{c}0.14 \\
(0.07-0.3)\end{array}$ & $\begin{array}{c}0.14 \\
(0.08-0.24)\end{array}$ & $\begin{array}{c}0.19 \\
(0.13-0.28)\end{array}$ & $\begin{array}{c}0.27 \\
(0.17-0.34)\end{array}$ \\
\hline Nickel & $\begin{array}{c}\mathrm{BD} \\
(<0.01)\end{array}$ & $\mathrm{BD}$ & $\begin{array}{c}0.03 \\
(0.01-0.06)\end{array}$ & $\begin{array}{c}0.02 \\
(B D-0.03)\end{array}$ & $\begin{array}{c}0.02 \\
(0.02-0.03)\end{array}$ & $\begin{array}{c}0.03 \\
(0.02-0.04)\end{array}$ \\
\hline Silicon & $\begin{array}{c}5.0 \\
(4.2-6.1)\end{array}$ & $\begin{array}{c}5.2 \\
(4.1-6)\end{array}$ & $\begin{array}{c}5.2 \\
(3.1-7.6)\end{array}$ & $\begin{array}{c}6.0 \\
(3.1-13)\end{array}$ & $\begin{array}{c}5.2 \\
(3.4-9.8)\end{array}$ & $\begin{array}{c}8.1 \\
(3.7-17)\end{array}$ \\
\hline Sodium & $\begin{array}{c}1 \\
(0.7-2.0)\end{array}$ & ${ }^{3}(2.1-3.8)$ & $\begin{array}{c}16 \\
(2.8-41)\end{array}$ & $\begin{array}{c}18 \\
(7.1-38)\end{array}$ & $\begin{array}{c}14 \\
(5.5-26)\end{array}$ & $\begin{array}{c}10 \\
(3.7-17)\end{array}$ \\
\hline Sulfate & $\stackrel{5}{(2-7)}$ & $\begin{array}{c}21 \\
(14-27)\end{array}$ & $\begin{array}{c}52 \\
(2.8-41)\end{array}$ & $\begin{array}{c}61 \\
(33-107)\end{array}$ & $\begin{array}{c}58 \\
(27-90)\end{array}$ & $\begin{array}{c}48 \\
(24-75)\end{array}$ \\
\hline Suspended solids & $\begin{array}{c}20 \\
(6-46)\end{array}$ & $\begin{array}{c}11 \\
(B D-30)\end{array}$ & $\begin{array}{c}27 \\
(\mathrm{BD}-88)\end{array}$ & $\begin{array}{c}41 \\
(B D-185)\end{array}$ & $\begin{array}{c}43 \\
(4-222)\end{array}$ & $\begin{array}{c}74 \\
(6-319)\end{array}$ \\
\hline
\end{tabular}

${ }^{\circ} \mathrm{BD}=$ below detection.

Source: M. A. McGaha, 1989, Toxicity Monitoring at ORGDP July-September 1988, Report No. K/QT-342, Oak Ridge Gaseous Diffusion Plant, Oak Ridge, Tennessee.

Note: Seven samples were taken from each location. 
Table B.9. Mean (range in parentheses) concentrations of selected parameters (in milligrams per liter) in Mitchell Branch water obtained by the Oak Ridge K-25 Site Process

Support Department in conjunction with the toxicity test conducted during December 1989

\begin{tabular}{|c|c|c|c|c|c|c|}
\hline \multirow[b]{2}{*}{ Parameter } & \multicolumn{6}{|c|}{ Mitchell Branch kilometer } \\
\hline & 1.43 & 1.00 & 0.71 & 0.54 & 0.45 & 0.12 \\
\hline Aluminum & $\begin{array}{c}0.21 \\
(0.13-0.41)\end{array}$ & $\begin{array}{c}0.29 \\
(0.18-0.51)\end{array}$ & $\begin{array}{c}0.22 \\
0.12-0.33)\end{array}$ & $\begin{array}{c}0.26 \\
(0.17-0.38)\end{array}$ & $\begin{array}{c}0.20 \\
(0.15-0.31)\end{array}$ & $\begin{array}{c}0.46 \\
(0.24-0.65)\end{array}$ \\
\hline Calcium & $\begin{array}{c}14 \\
(13-15)\end{array}$ & $\begin{array}{c}26 \\
(24-28)\end{array}$ & $\begin{array}{c}40 \\
(32-65)\end{array}$ & $\begin{array}{c}46 \\
(37-66)\end{array}$ & $\begin{array}{c}48 \\
(39-70)\end{array}$ & $\begin{array}{c}54 \\
(44-76)\end{array}$ \\
\hline Chloride & $\begin{array}{c}1 \\
(1.1-1.2)\end{array}$ & $\begin{array}{c}2 \\
(1.5-3)\end{array}$ & $\begin{array}{c}23 \\
(8-91)\end{array}$ & $\begin{array}{c}26 \\
(9.4-79)\end{array}$ & $\begin{array}{c}28 \\
(12-82)\end{array}$ & $\begin{array}{c}34 \\
(18-87)\end{array}$ \\
\hline Dissolved solids & $\begin{array}{c}51 \\
(34-82)\end{array}$ & $\begin{array}{c}136 \\
(108-162)\end{array}$ & $\begin{array}{c}238 \\
(159-456)\end{array}$ & $\begin{array}{c}259 \\
(178-424)\end{array}$ & $\begin{array}{c}273 \\
(206-444)\end{array}$ & $\begin{array}{c}302 \\
(230-468)\end{array}$ \\
\hline Fluoride & $\begin{array}{l}\mathrm{BD}^{a} \\
(<0.1)\end{array}$ & BD & BD & BD & BD & \\
\hline Iron & $\begin{array}{c}0.29 \\
(0.22-0.45)\end{array}$ & $\begin{array}{c}0.32 \\
(0.23-0.46)\end{array}$ & $\begin{array}{c}0.21 \\
(0.14-0.35)\end{array}$ & $\begin{array}{c}0.26 \\
(0.19-0.39)\end{array}$ & $\begin{array}{c}0.35 \\
(0.31-0.45)\end{array}$ & $\begin{array}{c}0.7 \\
(0.44-0.94)\end{array}$ \\
\hline Magnesium & $\begin{array}{c}7.6 \\
(6.8-8.2)\end{array}$ & $\begin{array}{c}9.8 \\
(8.9-11)\end{array}$ & $\begin{array}{c}10.2 \\
(9.1-13)\end{array}$ & $\begin{array}{c}11 \\
(9.5-13)\end{array}$ & $\begin{array}{c}11 \\
(10-13)\end{array}$ & $\begin{array}{c}12 \\
(11-14)\end{array}$ \\
\hline Manganese & $\begin{array}{c}0.13 \\
(0.12-0.14)\end{array}$ & $\begin{array}{c}0.14 \\
(0.13-0.18)\end{array}$ & $\begin{array}{c}0.08 \\
(0.07-0.1)\end{array}$ & $\begin{array}{c}0.15 \\
(0.14-0.17)\end{array}$ & $\begin{array}{c}0.22 \\
(0.19-0.24)\end{array}$ & $\begin{array}{c}0.28 \\
(0.23-0.31)\end{array}$ \\
\hline Nickel & $\begin{array}{c}\text { BD } \\
(<0.01)\end{array}$ & $\begin{array}{c}0.01 \\
\text { BD-0.03) }\end{array}$ & $\begin{array}{c}0.01 \\
(B D-0.02)\end{array}$ & BD & $\begin{array}{c}0.02 \\
(0.02-0.04)\end{array}$ & $\begin{array}{c}0.02 \\
(0.02-0.05)\end{array}$ \\
\hline Silicon & $\begin{array}{c}3.9 \\
(3.6-5)\end{array}$ & $\begin{array}{c}4.0 \\
(3.7-4.4)\end{array}$ & $\begin{array}{c}3.2 \\
(2.9-3.5)\end{array}$ & $\begin{array}{c}3.6 \\
(3.2-4)\end{array}$ & $\begin{array}{c}3.3 \\
(3-3.7)\end{array}$ & $\begin{array}{c}3.6 \\
(3.2-4)\end{array}$ \\
\hline Sodium & $\begin{array}{c}1 \\
(0.9-1.1)\end{array}$ & $\begin{array}{c}2 \\
(1.7-2.0)\end{array}$ & $\begin{array}{c}16 \\
(6.6-62)\end{array}$ & $\begin{array}{c}21 \\
(8.2-58)\end{array}$ & $\begin{array}{c}19 \\
(7.5-53)\end{array}$ & $\begin{array}{c}21 \\
(10-56)\end{array}$ \\
\hline Sulfate & $\begin{array}{c}4 \\
(3.2-4.1)\end{array}$ & $\begin{array}{c}11 \\
(10-15)\end{array}$ & $\begin{array}{c}41 \\
(21-124)\end{array}$ & $\begin{array}{c}49 \\
(26-102)\end{array}$ & $\begin{array}{c}54 \\
(30-111)\end{array}$ & $\begin{array}{c}55 \\
(29-114)\end{array}$ \\
\hline Suspended solids & $\begin{array}{c}7.4 \\
(4-12)\end{array}$ & $\begin{array}{c}6 \\
(2-8)\end{array}$ & $\begin{array}{c}2 \\
(\mathrm{BD}-2)\end{array}$ & $\begin{array}{c}4.9 \\
(2-10)\end{array}$ & $\begin{array}{c}6.8 \\
(2-12)\end{array}$ & $\begin{array}{c}13 \\
(5-22)\end{array}$ \\
\hline
\end{tabular}

${ }^{a} \mathrm{BD}=$ below detection.

Source: J. L. Shoemaker, 1990, Toxicity Monitoring at ORGDP October-December 1989, Report No. K/QT-373, Oak Ridge Gaseous Diffusion Plant, Oak Ridge, Tennessee.

Note: Seven samples were taken from each location. 
Appendix C

\section{RESULTS OF QUALITY ASSURANCE/QUALITY CONTROL ANALYSES OF FISH SAMPLES}




\section{RESULTS OF QUALITY ASSURANCE/QUALITY CONTROL ANALYSES OF FISH SAMPLES}

Ten pairs of duplicate samples of fish muscle were analyzed for mercury. The mean coefficient of variation (CV) between sample pairs was $21 \%$, with a mean standard deviation (SD) of $0.07 \mu \mathrm{g} / \mathrm{g}$. The mean absolute difference between duplicate samples was $0.10 \mu \mathrm{g} / \mathrm{g}$. Multiple analyses of EPA reference fish $(n=6)$ averaged $( \pm S D) 2.54 \pm 0.09 \mu \mathrm{g} / \mathrm{g}$, vs an expected value of $2.52 \mu \mathrm{g} / \mathrm{g}$. Mercury concentrations in sunfish $(n=10)$ from reference sites averaged $0.07 \pm 0.02 \mu \mathrm{g} / \mathrm{g}$.

The results of PCB analyses were somewhat more variable than those from mercury analyses. The mean coefficient of variation between 11 sample pairs was $36 \%$, with a mean standard deviation of $0.15 \mu \mathrm{g} / \mathrm{g}$. The mean absolute differ- ence between sample pairs was $0.36 \mu \mathrm{g} / \mathrm{g}$. Recoveries of mixtures of PCB-1254 and PCB-1260 spiked into samples of uncontaminated fish and clams averaged $105 \pm 9 \%$. PCB concentrations in fish $(n=11)$ from the uncontaminated reference sites averaged $0.05 \pm 0.05 \mu \mathrm{g} / \mathrm{g}$.

Other quality assurance samples, includi' g split sample exchanges among laboraturies at TVA, Tennessee Department of Health and Environment, and EPA, are run as part of BMAP studies for ORNL and the Y-12 Plant. Results of these evaluations are applicable to K-25 site samples and are available in Loar (1991b, 1992, 1993) and Hinzman et al. 1993. 
Appendix D

CONCENTRATIONS OF MERCURY AND PCBs

IN INDIVIDUAL FISH FROM MITCHELL

BRANCH, POPLAR CREEK, AND

NEARBY REACHES OF THE

CLINCH RIVER 
Table D.1. Concentrations of mercury and PCBs (micrograms per gram wet weight) in resident fish from Mitchell Branch and nearby reaches of Poplar Creek and the Clinch River, 1987-1990

\begin{tabular}{|c|c|c|c|c|c|c|c|c|c|c|}
\hline Site & Date & Spp & Sex & No.d & $W t^{\circ}$ & Lgth' & $\mathrm{Hg}_{\mathrm{g}}$ & $\mathrm{PCB}^{2}$ & $1254^{h}$ & $1260^{\prime}$ \\
\hline PCK 6.9 & $06 / 01 / 87$ & REDBRE & $\mathbf{M}$ & 7025 & 141.5 & 19.5 & 0.38 & 0.46 & 0.17 & 0.29 \\
\hline PCK 6.9 & $06 / 01 / 87$ & REDBRE & $\mathbf{F}$ & 9177 & 48.4 & 13.6 & 0.19 & 0.18 & 0.06 & 0.12 \\
\hline PCK 6.9 & $06 / 01 / 87$ & REDBRE & $\mathbf{F}$ & 9157 & 41.0 & 13.3 & 0.27 & 0.34 & 0.16 & 0.18 \\
\hline PCK 6.9 & $06 / 01 / 87$ & REDBRE & $\mathbf{F}$ & 7638 & 15.2 & 9.6 & & 0.40 & 0.12 & 0.28 \\
\hline PCK 6.9 & $06 / 01 / 87$ & BLUGIL & $\mathbf{F}$ & 7052 & 93.0 & 17.6 & 0.57 & 0.32 & 0.13 & 0.19 \\
\hline PCK 6.9 & $06 / 01 / 87$ & BLUGIL & $\mathbf{F}$ & 7054 & 71.1 & 15.6 & 0.57 & 0.05 & 0.03 & 0.02 \\
\hline PCK 6.9 & $06 / 01 / 87$ & BLUGIL & $\mathbf{M}$ & 7061 & 102.1 & 17.0 & 0.38 & 0.19 & 0.04 & 0.15 \\
\hline PCK 6.9 & 06/01/87 & BLUGIL & $\mathbf{M}$ & 7056 & 85.3 & 16.7 & 0.24 & 0.09 & 0.02 & 0.07 \\
\hline PCK 6.9 & 06/01/87 & BLUGIL & $\mathbf{F}$ & 7057 & 104.2 & 17.3 & 0.65 & 0.53 & 0.15 & 0.38 \\
\hline PCK 6.9 & $06 / 01 / 87$ & BLUGIL & $\mathbf{F}$ & 7055 & 63.5 & 15.3 & 0.27 & 0.29 & 0.18 & 0.11 \\
\hline PCK 6.9 & $06 / 01 / 87$ & BLUGIL & $\mathbf{F}$ & 7077 & 75.5 & 15.9 & 0.38 & 0.14 & 0.07 & 0.07 \\
\hline PCK 6.9 & $06 / 01 / 87$ & BLUGIL & $\mathbf{F}$ & 7078 & 42.8 & 13.8 & 0.33 & 0.11 & 0.03 & 0.08 \\
\hline PCK 10.4 & $11 / 18 / 87$ & BLUGIL & $\mathbf{M}$ & 6348 & 77.2 & 16.0 & 0.05 & 0.11 & 0.03 & 0.08 \\
\hline PCK 10.4 & $11 / 18 / 87$ & BLUGIL & $\mathbf{F}$ & 6349 & 59.1 & 15.0 & 0.13 & 0.04 & 0.02 & 0.02 \\
\hline PCK 10.4 & $11 / 18 / 87$ & BLUGIL & $\mathbf{F}$ & 6350 & 72.0 & 16.0 & 0.09 & 0.04 & 0.02 & 0.02 \\
\hline PCK 10.4 & $11 / 18 / 87$ & BLUGIL & $\mathbf{F}$ & 6351 & 71.3 & 15.0 & 0.10 & 0.07 & 0.03 & 0.04 \\
\hline PCK 10.4 & $11 / 18 / 87$ & BLUGIL & $\mathbf{F}$ & 6352 & 39.0 & 13.5 & 0.09 & 0.03 & 0.02 & 0.01 \\
\hline PCK 10.4 & $11 / 18 / 87$ & BLUGIL & $\mathbf{M}$ & 6353 & 37.7 & 12.8 & 0.10 & 0.06 & 0.02 & 0.04 \\
\hline PCK 10.4 & $11 / 18 / 87$ & BLUGIL & $\mathbf{F}$ & 6354 & 45.4 & 13.5 & 0.16 & 0.08 & 0.04 & 0.04 \\
\hline PCK 10.4 & $11 / 18 / 87$ & BLUGIL & $\mathbf{F}$ & 6355 & 64.6 & 15.4 & 0.11 & 0.02 & 0.01 & 0.02 \\
\hline PCK 8.2 & $11 / 18 / 87$ & BLUGIL & $\mathbf{M}$ & 6340 & 59.4 & 14.6 & 0.22 & 0.21 & 0.13 & 0.08 \\
\hline PCK 8.2 & $11 / 18 / 87$ & BLUGIL & $\mathbf{F}$ & 6341 & 45.9 & 14.2 & 0.69 & 0.25 & 0.10 & 0.15 \\
\hline PCK 8.2 & $11 / 18 / 87$ & BLUGIL & $\mathbf{M}$ & 6342 & 28.1 & 11.8 & 0.38 & 0.27 & 0.12 & 0.15 \\
\hline PCK 8.2 & $11 / 18 / 87$ & BLUGIL & $\mathbf{F}$ & 6343 & 40.5 & 13.2 & 0.51 & 0.15 & 0.06 & 0.09 \\
\hline PCK 8.2 & $11 / 18 / 87$ & BLUGIL & $\mathbf{F}$ & 6344 & 84.1 & 17.3 & 0.60 & 0.14 & 0.07 & 0.07 \\
\hline PCK 8.2 & $11 / 18 / 87$ & BLUGIL & $\mathbf{M}$ & 6345 & 91.5 & 17.2 & 0.33 & 0.36 & 0.12 & 0.24 \\
\hline PCK 8.2 & $11 / 18 / 87$ & BLUGIL & $F$ & 6346 & 57.0 & 14.7 & 0.50 & 0.12 & 0.04 & 0.08 \\
\hline PCK 8.2 & $11 / 18 / 87$ & BLUGIL & $\mathbf{F}$ & 6347 & 66.5 & 15.3 & 0.21 & 0.11 & 0.05 & 0.06 \\
\hline PCK 6.9 & $11 / 18 / 87$ & BLUGIL & $\mathbf{M}$ & 6332 & 60.7 & 15.0 & 0.28 & 0.22 & 0.12 & 0.10 \\
\hline PCK 6.9 & $11 / 18 / 87$ & BLUGIL & & 6333 & 41.7 & 13.9 & 0.66 & 0.35 & 0.01 & 0.34 \\
\hline PCK 6.9 & $11 / 18 / 87$ & BLUGIL & $\mathbf{M}$ & 6334 & 45.3 & 13.5 & 0.50 & 0.20 & 0.11 & 0.09 \\
\hline PCK 6.9 & $11 / 18 / 87$ & BLUGIL & $\mathbf{M}$ & 6335 & 55.9 & 14.4 & 0.42 & 0.25 & 0.12 & 0.13 \\
\hline PCK 6.9 & $11 / 18 / 87$ & BLUGIL & $\mathbf{M}$ & 6336 & 48.6 & 14.2 & 0.26 & 0.18 & 0.05 & 0.13 \\
\hline PCK 6.9 & $11 / 18 / 87$ & BLUGIL & $\mathbf{M}$ & 6337 & 71.6 & 15.4 & 0.33 & 0.29 & 0.13 & 0.16 \\
\hline PCK 6.9 & $11 / 18 / 87$ & BLUGIL & $\mathbf{F}$ & 6338 & 70.7 & 15.8 & 0.50 & 0.14 & 0.06 & 0.08 \\
\hline PCK 6.9 & 11/18/87 & BLUGIL & $\mathbf{F}$ & 6339 & 95.9 & 17.2 & 0.48 & 0.47 & 0.09 & 0.38 \\
\hline PCK 1.6 & $12 / 08 / 87$ & BLUGIL & $\mathbf{M}$ & 7043 & 135.9 & 18.9 & 0.22 & 0.17 & 0.06 & 0.11 \\
\hline PCK 1.6 & $12 / 08 / 87$ & BLUGIL & $\mathbf{M}$ & 7044 & 97.9 & 18.0 & 0.35 & 0.18 & 0.08 & 0.10 \\
\hline PCK 1.6 & $12 / 08 / 87$ & BLUGIL & $\mathbf{M}$ & 7045 & 85.8 & 16.7 & 0.30 & 0.13 & 0.05 & 0.08 \\
\hline PCK 1.6 & $12 / 08 / 87$ & BLUGIL & $\mathbf{F}$ & 7046 & 76.1 & 16.2 & 0.06 & 0.10 & 0.06 & 0.04 \\
\hline PCK 1.6 & $12 / 08 / 87$ & BLUGIL & $\mathbf{F}$ & 7047 & 56.2 & 15.2 & 0.11 & 0.17 & 0.08 & 0.09 \\
\hline PCK 1.6 & $12 / 08 / 87$ & BLUGIL & $\mathbf{M}$ & 7048 & 99.5 & 18.2 & 0.09 & 0.14 & 0.02 & 0.12 \\
\hline PCK 1.6 & $12 / 08 / 87$ & BLUGIL & $\mathbf{M}$ & 7049 & 58.7 & 16.3 & 0.07 & 0.37 & 0.11 & 0.28 \\
\hline PCK 1.6 & $12 / 08 / 87$ & BLUGIL & $\mathbf{M}$ & 7050 & 109.5 & 18.8 & 0.06 & 0.12 & 0.03 & 0.09 \\
\hline
\end{tabular}


Table D.1 (continued)

\begin{tabular}{|c|c|c|c|c|c|c|c|c|c|c|}
\hline Site & Date & Spp & Sex & No.d & $W^{\prime}$ & Lgth' & $\mathrm{Hg}$ & $\mathrm{PCB}$ & $1254^{4}$ & $1260^{\circ}$ \\
\hline CRK 15.0 & $11 / 18 / 87$ & BLUGIL & $\mathbf{F}$ & 6324 & 57.3 & 15.3 & 0.10 & 0.06 & 0.03 & 0.03 \\
\hline CRK 15.0 & $11 / 18 / 87$ & BLUGIL & $\mathbf{M}$ & 6325 & 73.6 & 15.9 & 0.11 & 0.10 & 0.03 & 0.07 \\
\hline CRK 15.0 & $11 / 18 / 87$ & BLUGIL & $\mathbf{M}$ & 6326 & 33.9 & 12.8 & 0.06 & 0.07 & 0.04 & 0.03 \\
\hline CRK 15.0 & $11 / 18 / 87$ & BLUGIL & $\mathbf{F}$ & 6327 & 65.0 & 15.1 & 0.11 & 0.06 & 0.03 & 0.03 \\
\hline CRK 15.0 & $11 / 18 / 87$ & BLUGIL & $M$ & 6328 & 57.6 & 14.8 & 0.11 & 0.06 & 0.02 & 0.04 \\
\hline CRK 15.0 & 11/18/87 & BLUGIL & $\mathbf{F}$ & 6329 & 65.1 & 15.3 & 0.31 & 0.04 & 0.01 & 0.03 \\
\hline CRK 15.0 & $11 / 18 / 87$ & BLUGIL & $\mathbf{M}$ & 6330 & 67.4 & 15.5 & 0.10 & 0.06 & 0.02 & 0.04 \\
\hline CRK 15.0 & $11 / 18 / 87$ & BLUGIL & $\mathbf{M}$ & 6331 & 69.5 & 15.8 & 0.23 & 0.15 & 0.01 & 0.14 \\
\hline MHL 38 & $11 / 11 / 87$ & BLUGIL & $\mathbf{M}$ & 6308 & 72.2 & 15.9 & 0.03 & 0.03 & 0.02 & 0.01 \\
\hline MHL 38 & $11 / 11 / 87$ & BLUGIL & $F$ & 6309 & 51.9 & 15.0 & 0.10 & 0.02 & 0.02 & 0.01 \\
\hline MHL 38 & $11 / 11 / 87$ & BLUGIL & $\mathbf{M}$ & 6310 & 53.9 & 14.8 & 0.03 & 0.06 & 0.06 & 0.01 \\
\hline MHL 38 & $11 / 11 / 87$ & BLUGIL & $\mathbf{M}$ & 6311 & 61.0 & 14.8 & 0.03 & 0.03 & 0.01 & 0.02 \\
\hline MHL 38 & $11 / 11 / 87$ & BLUGIL & $\mathbf{F}$ & 6312 & 34.7 & 13.0 & 0.03 & 0.02 & 0.02 & 0.01 \\
\hline MHL 38 & $11 / 11 / 87$ & BLUGIL & $\mathbf{M}$ & 6313 & 31.3 & 12.5 & 0.03 & 0.02 & 0.01 & 0.01 \\
\hline MHL 38 & $11 / 11 / 87$ & BLUGIL & $\mathbf{F}$ & 6314 & 108.8 & 18.0 & 0.04 & 0.02 & 0.02 & 0.01 \\
\hline MHL 38 & $11 / 11 / 87$ & BLUGIL & $\mathbf{M}$ & 6315 & 72.8 & 16.1 & 0.02 & 0.04 & 0.03 & 0.01 \\
\hline PCK 10.4 & $11 / 16 / 88$ & BLUGIL & $\mathbf{M}$ & 7594 & 88.1 & 16.6 & 0.03 & 0.01 & 0.01 & 0.001 \\
\hline PCK 10.4 & $11 / 16 / 88$ & BLUGIL & $\mathbf{F}$ & 7595 & 66.5 & 15.5 & 0.05 & 0.01 & 0.001 & 0.01 \\
\hline PCK 10.4 & $11 / 16 / 88$ & BLUGIL & $\mathbf{M}$ & 7590 & 105.4 & 18.3 & 0.05 & 0.02 & 0.02 & 0.001 \\
\hline PCK 10.4 & $11 / 16 / 88$ & BLUGIL & $\mathbf{F}$ & 7591 & 54.5 & 14.6 & 0.24 & 0.26 & 0.13 & 0.13 \\
\hline PCK 10.4 & $11 / 16 / 88$ & BLUGIL & $\mathbf{F}$ & 7593 & 73.2 & 15.7 & 0.03 & 0.03 & 0.03 & 0.001 \\
\hline PCK 10.4 & $11 / 16 / 88$ & BLUGIL & $\mathbf{M}$ & 7597 & 90.0 & 16.0 & 0.05 & 0.03 & 0.02 & 0.01 \\
\hline PCK 10.4 & $11 / 16 / 88$ & BLUGIL & $\mathbf{M}$ & 7592 & 72.1 & 15.6 & 0.04 & 0.01 & 0.01 & 0.001 \\
\hline PCK 10.4 & $11 / 16 / 88$ & BLUGIL. & $\mathbf{M}$ & 7596 & 67.9 & 15.8 & 0.13 & 0.01 & 0.001 & 0.01 \\
\hline PCK 8.2 & $11 / 16 / 88$ & BLUGIL & $\mathbf{M}$ & 7580 & 80.8 & 16.2 & 0.23 & 0.59 & 0.45 & 0.14 \\
\hline PCK 8.2 & $11 / 16 / 88$ & BLUGIL & $\mathbf{M}$ & 7581 & 112.3 & 17.8 & 0.33 & 0.55 & 0.49 & 0.06 \\
\hline PCK 8.2 & $11 / 16 / 88$ & BLUGIL & $\mathbf{M}$ & 7582 & 86.3 & 16.8 & 0.34 & 0.49 & 0.40 & 0.09 \\
\hline PCK 8.2 & $11 / 16 / 88$ & BLUGIL & $\mathbf{M}$ & 7583 & 63.8 & 15.0 & 0.40 & 0.18 & 0.14 & 0.04 \\
\hline PCK 8.2 & $11 / 16 / 88$ & BLUGIL & $\mathbf{F}$ & 7584 & 75.7 & 16.0 & 0.54 & 0.22 & 0.13 & 0.09 \\
\hline PCK 8.2 & $11 / 16 / 88$ & BLUGIL & $\mathbf{M}$ & 7585 & 63.8 & 15.0 & 0.32 & 0.11 & 0.07 & 0.04 \\
\hline PCK 8.2 & $11 / 16 / 88$ & BLUGIL & $\mathbf{M}$ & 7586 & 69.1 & 15.2 & 0.54 & 0.24 & 0.17 & 0.07 \\
\hline PCK 8.2 & $11 / 16 / 88$ & BLUGIL & $\mathbf{F}$ & 7587 & 98.4 & 17.4 & 0.59 & 0.08 & 0.05 & 0.03 \\
\hline PCK 6.9 & $11 / 16 / 88$ & BLUGIL & $\mathbf{F}$ & 7570 & 73.4 & 16.6 & 0.54 & 0.17 & 0.14 & 0.03 \\
\hline PCK 6.9 & $11 / 16 / 88$ & BLUGIL & $\mathbf{M}$ & 7571 & 93.9 & 17.1 & 0.09 & 0.14 & 0.12 & 0.02 \\
\hline PCK 6.9 & $11 / 16 / 88$ & BLUGIL & $\mathbf{M}$ & 7572 & 54.7 & 15.0 & 0.28 & & & \\
\hline PCK 6.9 & $11 / 16 / 88$ & BLUGIL & $\mathbf{M}$ & 7573 & 49.6 & 14.2 & 0.36 & 0.39 & 0.25 & 0.14 \\
\hline PCK 6.9 & $11 / 16 / 88$ & BLUGIL & F & 7574 & 51.2 & 14.5 & 0.39 & 0.17 & 0.12 & 0.05 \\
\hline PCK 6.9 & $11 / 16 / 88$ & BLUGIL & $\mathbf{M}$ & 7575 & 67.9 & 15.8 & 0.31 & 0.40 & 0.23 & 0.17 \\
\hline PCK 6.9 & $11 / 16 / 88$ & BLUGIL & $\mathbf{M}$ & 7576 & 60.2 & 15.3 & 0.09 & 0.08 & 0.06 & 0.02 \\
\hline PCK 6.9 & $11 / 16 / 88$ & BLUGIL & $\mathbf{F}$ & 7577 & 50.2 & 14.2 & 0.27 & 0.15 & 0.12 & 0.03 \\
\hline PCK 1.6 & $11 / 16 / 88$ & BLUGIL & F & 7650 & 68.2 & 15.9 & 0.24 & 0.14 & 0.11 & 0.03 \\
\hline PCK 1.6 & $11 / 16 / 88$ & BLUGIL & $\mathbf{F}$ & 7651 & 49.2 & 14.6 & 0.24 & 0.23 & 0.16 & 0.07 \\
\hline PCK 1.6 & $11 / 16 / 88$ & BLUGIL & $\mathbf{M}$ & 7652 & 78.8 & 16.0 & 0.15 & 0.30 & 0.26 & 0.04 \\
\hline PCK 1.6 & $11 / 16 / 88$ & BLUGIL & $\mathbf{M}$ & 7653 & 55.2 & 14.2 & 0.14 & 0.39 & 0.37 & 0.02 \\
\hline PCK 1.6 & $11 / 16 / 88$ & BLUGIL & $\mathbf{M}$ & 7654 & 70.0 & 15.5 & 0.06 & 0.06 & 0.05 & 0.01 \\
\hline PCK 1.6 & $11 / 16 / 88$ & BLUGIL & $\mathrm{F}$ & 7655 & 59.2 & 14.8 & 0.16 & 0.11 & 0.09 & 0.02 \\
\hline
\end{tabular}


Tuble D.1 (continued)

\begin{tabular}{|c|c|c|c|c|c|c|c|c|c|c|}
\hline Siten & Date & Spp & Sex & No.d & $W t^{\circ}$ & Lgth' & $\mathbf{H}_{8}$ & PCBe & $1254^{\prime \prime}$ & $1260^{\circ}$ \\
\hline $\begin{array}{l}\text { PCK } 1.6 \\
\text { PCK } 1.6\end{array}$ & $\begin{array}{l}11 / 16 / 88 \\
11 / 16 / 88\end{array}$ & $\begin{array}{l}\text { BLUGIL. } \\
\text { BL.UGIL. }\end{array}$ & $\begin{array}{l}\mathbf{M} \\
\mathbf{F}\end{array}$ & $\begin{array}{l}7656 \\
7657\end{array}$ & $\begin{array}{l}59.6 \\
61.2\end{array}$ & $\begin{array}{l}14.6 \\
14.5\end{array}$ & $\begin{array}{l}0.11 \\
0.22\end{array}$ & $\begin{array}{l}0.18 \\
0.14\end{array}$ & $\begin{array}{l}0.12 \\
0.09\end{array}$ & $\begin{array}{l}0.06 \\
0.05\end{array}$ \\
\hline CRK 15.0 & $11 / 16 / 88$ & BLUGIL & $\mathbf{M}$ & 7643 & 90.5 & 17.5 & 0.08 & 0.06 & 0.03 & 0.03 \\
\hline CRK 15.0 & $11 / 16 / 88$ & BLUGIL & $\mathbf{M}$ & 7648 & 69.9 & 15.6 & 0.10 & 0.12 & 0.08 & 0.04 \\
\hline CRK 15.0 & $11 / 16 / 88$ & BLUGIL & $\mathbf{M}$ & 7647 & 53.5 & 14.5 & 0.02 & 0.17 & 0.15 & 0.02 \\
\hline CRK 15.0 & $11 / 16 / 88$ & BLUGIL & $M$ & 7641 & 83.6 & 16.2 & 0.16 & 0.01 & 0.001 & 0.01 \\
\hline CRK 15.0 & $11 / 16 / 88$ & BLUGIL & $\mathbf{M}$ & 7640 & 54.8 & 15.0 & 0.20 & 0.22 & 0.22 & 0.001 \\
\hline CRK 15.0 & $11 / 16 / 88$ & BLUGIL & $\mathbf{M}$ & 7645 & 104.5 & 17.8 & 0.16 & 0.17 & 0.16 & 0.01 \\
\hline CRK 15.0 & $11 / 16 / 88$ & BLUGIL & $\mathbf{M}$ & 7642 & 109.6 & 17.8 & 0.28 & 0.08 & 0.02 & 0.06 \\
\hline CRK 15.0 & $11 / 16 / 88$ & BLUGIL & M & 7644 & 78.2 & 16.4 & 0.11 & 0.05 & 0.03 & 0.02 \\
\hline PCK 6.9 & 03/07/90 & BLUGIL & $\mathbf{M}$ & 5230 & 80.3 & 17.3 & 0.68 & 0.10 & 0.001 & 0.10 \\
\hline PCK 6.9 & 03/07/90 & BLUGIL & $\mathbf{M}$ & 5231 & 63.4 & 16.0 & 0.43 & 0.12 & 0.04 & 0.08 \\
\hline PCK 6.9 & $03 / 07 / 90$ & BLUGIL & $\mathbf{M}$ & 5232 & 114.8 & 19.4 & 0.33 & 0.17 & 0.001 & 0.17 \\
\hline PCK 6.9 & 03/07/90 & BLUGIL & $\mathbf{M}$ & 5233 & 69.4 & 16.4 & 0.43 & 0.14 & 0.07 & 0.07 \\
\hline PCK 6.9 & 03/07/90 & BLUGIL & $\mathbf{M}$ & 5234 & 42.6 & 14.2 & 0.33 & 0.07 & 0.03 & 0.04 \\
\hline PCK 6.9 & 03/07/90 & BLUGIL & $\mathbf{M}$ & 5235 & 83.1 & 17.4 & 0.42 & 0.07 & 0.001 & 0.07 \\
\hline PCK 6.9 & 03/07/90 & BLUGIL & $\mathbf{M}$ & 5236 & 59.2 & 15.5 & 0.30 & 0.15 & 0.09 & 0.06 \\
\hline PCK 6.9 & 03/07/90 & BLUGIL & M & 5237 & 58.5 & 15.4 & 0.51 & 0.17 & 0.05 & 0.12 \\
\hline PCK 8.2 & 03/07/90 & BLUGIL & $\mathbf{M}$ & 5240 & 71.0 & 16.3 & 0.14 & 0.08 & 0.03 & 0.05 \\
\hline PCK 8.2 & 03/07/90 & BLUGIL & $\mathbf{M}$ & 5241 & 70.7 & 16.0 & 0.33 & 0.07 & 0.03 & 0.04 \\
\hline PCK 8.2 & 03/07/90 & BLUGIL & $\mathbf{M}$ & 5242 & 89.5 & 17.4 & 0.43 & 0.02 & 0.001 & 0.02 \\
\hline PCK 8.2 & 03/07/90 & BLUGIL & $\mathrm{M}$ & 5243 & 77.5 & 16.7 & 0.30 & 0.06 & 0.02 & 0.04 \\
\hline PCK 8.2 & 03/07/90 & BLUGIL & F & 5244 & 65.4 & 15.9 & 0.65 & 0.05 & 0.02 & 0.03 \\
\hline PCK 8.2 & $03 / 07 / 90$ & BLUGIL & $\mathbf{M}$ & 5245 & 71.3 & 15.8 & 0.36 & 0.11 & 0.01 & 0.10 \\
\hline PCK 8.2 & 03/07/90 & BLUGIL & $\mathbf{M}$ & 5246 & 81.5 & 17.2 & 0.24 & 0.02 & 0.01 & 0.01 \\
\hline PCK 8.2 & 03/07/90 & BLUGIL & $\mathbf{M}$ & 5247 & 100.8 & 18.0 & 0.45 & 0.15 & 0.05 & 0.10 \\
\hline MIK 0.2 & 05/06/87 & REDBRE & I & MB1 & 5.7 & 6.8 & 0.18 & & & \\
\hline MIK 0.2 & 05/06/87 & REDBRE & I & MB2 & 8.6 & 8.0 & 0.16 & & & \\
\hline MIK 0.2 & $05 / 06 / 87$ & REDBRE & 1 & MB4 & 4.5 & 6.5 & 0.17 & & & \\
\hline MIK 0.2 & 05/06/87 & REDBRE & I & MB7 & 4.4 & 6.5 & 0.18 & & & \\
\hline MIK 0.2 & 05/06/87 & REDBRE & 1 & MB8 & 4.4 & 6.1 & 0.18 & & & \\
\hline MIK 0.2 & $05 / 06 / 87$ & REDBRE & 1 & MB10 & 3.3 & 5.9 & 0.15 & & & \\
\hline MIK 0.2 & $05 / 06 / 87$ & REDBRE & 1 & MB12 & 2.5 & 5.3 & 0.17 & & & \\
\hline MIK 0.2 & $03 / 23 / 88$ & BLUGIL & $\mathbf{F}$ & 7912 & 120.3 & 17.5 & 0.40 & & & \\
\hline MIK 0.2 & $03 / 23 / 88$ & REDBRE & $\mathbf{M}$ & 7913 & 9.3 & 7.9 & 0.09 & & & \\
\hline MIK 0.2 & 03/23/88 & REDBRE & 1 & 7914 & 4.0 & 5.9 & 0.13 & & & \\
\hline MIK 0.2 & $03 / 23 / 88$ & REDBRE & 1 & 7917 & 3.3 & 5.7 & 0.14 & & & \\
\hline MIK 0.2 & $03 / 23 / 88$ & REDBRE & 1 & 7921 & 3.3 & 5.5 & 0.12 & & & \\
\hline MIK 0.2 & 03/23/88 & REDBRE & I & 7922 & 3.1 & 5.5 & 0.11 & & & \\
\hline MIK 0.2 & 03/29/89 & REDBRE & $\mathbf{M}$ & 7377 & 10.4 & 8.1 & 0.12 & & & \\
\hline MIK 0.2 & 03/29/89 & REDBRE & $\mathbf{F}$ & 7378 & 3.0 & 5.2 & 0.22 & & & \\
\hline MIK 0.2 & $03 / 29 / 89$ & REDBRE & $\mathbf{M}$ & 7379 & 7.1 & 7.1 & 0.17 & & & \\
\hline MIK 0.2 & $03 / 29 / 89$ & REDBRE & $\mathbf{M}$ & 7479 & 37.8 & 13.0 & 0.20 & 3.27 & 2.73 & 0.54 \\
\hline MIK 0.2 & 03/29/89 & REDBRE & $\mathbf{F}$ & 7450 & 34.6 & 12.0 & 0.18 & 0.97 & 0.79 & 0.18 \\
\hline MIK 0.2 & $03 / 29 / 89$ & REDBRE & $\mathbf{M}$ & 7451 & 38.8 & 12.9 & 0.18 & 1.70 & 1.21 & 0.49 \\
\hline MIK 0.2 & 03/29/89 & REDBRF & $\mathbf{F}$ & 7453 & 36.3 & 12.9 & 0.21 & 1.93 & 1.52 & 0.41 \\
\hline MIK 0.2 & $03 / 29 / 89$ & REDBRE & F & 7454 & 46.7 & 13.0 & 0.22 & 0.50 & 0.38 & 0.12 \\
\hline MIK 0.2 & 03/29/89 & REDBRE & $\mathbf{M}$ & 7452 & 11.3 & 8.4 & & 2.41 & 2.22 & 0.19 \\
\hline
\end{tabular}


Table D.1 (continued)

\begin{tabular}{|c|c|c|c|c|c|c|c|c|c|c|}
\hline Site & Date & Spp & Sex & No. & $W^{\prime}$ & Lgeth' & $\mathrm{H}_{8}$ & PCB & $1254^{n}$ & $1260^{\circ}$ \\
\hline MIK 0.2 & 03/29/89 & REDBRE & $\mathbf{M}$ & 7455 & 19.1 & 9.9 & & 0.91 & 0.79 & 0.12 \\
\hline MIK 0.2 & 03/29/89 & REDBRE & $\mathbf{M}$ & 7456 & 32.1 & 11.6 & & 1.16 & 0.82 & 0.34 \\
\hline MIK 0.2 & $01 / 11 / 90$ & REDBRE & $\mathbf{M}$ & 5210 & 68.3 & 15.9 & 0.38 & 0.85 & 0.39 & 0.46 \\
\hline MIK 0.2 & $01 / 11 / 90$ & REDBRE & $\mathbf{F}$ & 5211 & 68.5 & 15.5 & 0.31 & 0.45 & 0.36 & 0.09 \\
\hline MIK 0.2 & $01 / 11 / 90$ & REDBRE & $\mathbf{M}$ & 5212 & 36.1 & 13.0 & 0.22 & 1.55 & 1.09 & 0.46 \\
\hline MIK 0.2 & $01 / 16 / 90$ & REDBRE & $\mathbf{F}$ & 5217 & 59.7 & 16.0 & 0.39 & 0.23 & 0.18 & 0.05 \\
\hline MIK 0.2 & $01 / 11 / 90$ & REDBRE & $\mathbf{M}$ & 5213 & 6.9 & 7.0 & 0.11 & & & \\
\hline MIK 0.2 & $01 / 16 / 90$ & REDBRE & $\mathbf{F}$ & 5200 & 38.6 & 13.4 & 0.26 & 0.71 & 0.51 & 0.20 \\
\hline MIK 0.2 & $01 / 16 / 90$ & REDBRE & $\mathbf{F}$ & 5201 & 29.5 & 12.6 & 0.25 & 0.87 & 0.57 & 0.30 \\
\hline MIK 0.2 & $01 / 16 / 90$ & REDBRE & $\mathbf{F}$ & 5218 & 20.7 & 11.8 & 0.21 & 0.74 & 0.57 & 0.17 \\
\hline MIK 0.2 & $01 / 16 / 90$ & REDBRE & $\mathbf{M}$ & 5202 & 15.0 & 9.9 & & 1.00 & 0.73 & 0.27 \\
\hline MIK 0.2 & $01 / 16 / 90$ & BLUGIL & $\mathbf{F}$ & 5219 & 72.6 & 16.5 & 0.31 & 0.74 & 0.46 & 0.28 \\
\hline
\end{tabular}

Site: PCK = Poplar Creek kjlometer, MIK = Mitchell Branch kjlometer, CRK = Clinch River kilometer, $\mathrm{MHL}=$ Meiton Hill Lake. Numerical designation is the distance from the mouth of the stream. Species (SPP): Bluegil = bluegill (Lepomis macrochirus), and Redbre = redbreast sunfish (Lepomis auritus)

Sex: $M=$ male, $F=$ female, and $I=$ immature.

No. = Fish identification number.

Wt = Fish weight, grams.

Lgth $=$ Fish total length, centimeters.

PCB = Total PCBs (sum of PCB-1254 and PCB-1260) in fish axial muscle, micrograms per gram wet weight.

1254 = PCB-1254 (Arochlor 1254) in fish axial muscle, micrograms per gram wet weight.

$' 1260$ = PCB-1260 (Arochlor 1260) in fish axial muscle, micrograms per gram wet weight. 
Table D.2. Detection limits of organic compounds (in micrograms per gram, wet weight)

Compound

Detection limit

\section{Capillary column GCMS}

Phenol

$<2.0$

Bis(2-chloroethyl) ether

$<2.0$

2-Chlorophenol

$<2.0$

1,3-Dichlorobenzene

$<2.0$

1,4-Dichlorobenzene

$<2.0$

Benzyl alcohol

$<2.0$

1,2-Dichlorobenzene

$<2.0$

2-Methylphenol

$<2.0$

Bis(2-chlorodisopropyl)ether

$<2.0$

4-Methylphenol

$<2.0$

$\mathrm{N}$-nitroso-di-N-propylamine

$<2.0$

Hexachloroethane

$<2.0$

Nitrobenzene

$<2.0$

Isophorone

$<2.0$

2-Nitrophenol

$<2.0$

2,4-Dimethylphenol

$<2.0$

Benzoic acid

$<10.0$

Bis(2-chloroethoxy)methane

$<2.0$

2,4-Dichlorophenol

$<2.0$

1,2,4-Trichlorobenzene

$<2.0$

Naphthalene

$<2.0$

4-Chloroaniline

$<2.0$

Hexachlorobutradiene

$<2.0$

4-Chloro-3-methylphenol

$<2.0$

2-Methylnaphthalene

$<2.0$

Hexachlorocyclapentadiene

$<2.0$

2,4,6-Trichlorophenol

$<2.0$

2,4,5-Trichlorophenol

$<10.0$

2-Chloronaphthalene

$<2.0$

2-Nitroaniline

$<10.0$

Dimethylphthalate

$<2.0$

Acenaphthalene

$<2.0$

3-Nitroaniline

$<10.0$

Acenaphthene

$<2.0$

2,4-Dinitrophenol

$<10.0$

Nitrophenol 
Table D.2. (continued)

\begin{tabular}{|c|c|}
\hline Compound & Detection limit ${ }^{a}$ \\
\hline Dibenzofuran & $<2.0$ \\
\hline 2,4-Dinitrotoluene & $<2.0$ \\
\hline 2,6-Dinitrotoluene & $<2.0$ \\
\hline Diethylphthalate & $<2.0$ \\
\hline 4-Chlorophenyl-phenylether & $<2.0$ \\
\hline Fluorene & $<2.0$ \\
\hline 4-Nitroaniline & $<10.0$ \\
\hline 4,6-Dinitro-2-methylphenol & $<10.0$ \\
\hline N-nitrosodiphenylamine & $<2.0$ \\
\hline 4-bromophenyl-phenylether & $<2.0$ \\
\hline Hexachlorobenzene & $<2.0$ \\
\hline Pentachlorophenol & $<10.0$ \\
\hline Phenanthrene & $<2.0$ \\
\hline Anthracene & $<2.0$ \\
\hline Di-N-butylphthalate & $<2.0$ \\
\hline Fluoranthene & $<2.0$ \\
\hline Pyrene & $<2.0$ \\
\hline Butylbenzylphthalate & $<2.0$ \\
\hline 3,3-Dichlorobenzidene & $<10.0$ \\
\hline Benz $[a]$ anthracene & $<2.0$ \\
\hline Bis(2-ethylhexyl)phthalate & $<2.0$ \\
\hline Chrysene & $<2.0$ \\
\hline Di- $N$-octylphthalate & $<2.0$ \\
\hline Benzo[b]fluoranthene & $<2.0$ \\
\hline Benzo[k]fluoranthene & $<2.0$ \\
\hline Benzo $[a]$ pyrene & $<2.0$ \\
\hline Indeno $[1,2,3-c d]$ pyrene & $<2.0$ \\
\hline Dibenz $[a, h]$ anthracene & $<2.0$ \\
\hline Benzo $[g, h, i]$ perylene & $<2.0$ \\
\hline \multicolumn{2}{|c|}{ Capillary column GC/ECD } \\
\hline Alpha-bhc & $<0.02$ \\
\hline Beta-bhc & $<0.04$ \\
\hline Delta-bhc & $<0.04$ \\
\hline Gamina-bhc & $<0.02$ \\
\hline Heptachlor & $<0.04$ \\
\hline Aldrin & $<0.04$ \\
\hline
\end{tabular}


Table D.2. (continued)

Compound

Detection limit

Heptachlor epoxide

$<0.04$

Endosulfan i

$<0.04$

Dieldrin

$<0.04$

4,4'-Dde

$<0.04$

Endrin

Endosulfan ii

$<0.2$

4,4'-Ddd

$<0.08$

$<0.2$

Endosulfan sulfate

$<0.2$

4,4'-Ddt

$<0.08$

Endrin ketone

$<0.4$

Methoxychlor

$<0.2$

Alpha chlordane

$<0.04$

Gamma chlordane

$<0.04$

Toxaphene

$<2$

HPLC with fluorescence detection

Naphthalene

$<0.12$

Acenaphthene

$<0.03$

Phenanthrene

$<0.01$

Anthracene

$<0.10$

Fluoranthene

$<0.2$

Pyrene

$<0.003$

Benz $[a \mid$ anthracene

$<0.001$

Benzo $[b \mid$ fluoranthene

$<0.03$

Benzo|k]fluoranthene

$<0.02$

Benzo[a]pyrene

$<0.004$

Dibenz $[a, h]$ anthracene

$<0.002$

Benzolg,h,i]perylene

$<0.005$

Indeno[1,2,3-cd]pyrene

$<1.0$

Chrysene

$<0.003$

${ }^{a}$ Optimum detection limit in 10-g sample. Sample weights varied between 5 and $10 \mathrm{~g}$, so detection limits were higher in some saınples. Concentrations of contaminants approximately ten times lower than these levels can be detected but are outside the limits of reliable quantification. 
Appendix E

CHECKLIST OF BENTHIC MACROINVERTEBRATE

TAXA COLLECTED FROM MITCHELL BRANCH AUGUST 1987 THROUGH JANUARY 1988 
Table E.1. Checklist of benthic macroinvertebrate tan collected from Mitchell Branch, August 1987 through January 1988

\begin{tabular}{|c|c|c|c|c|c|c|}
\hline \multirow[b]{2}{*}{ Taxon } & \multicolumn{6}{|c|}{ Site } \\
\hline & $\begin{array}{l}\text { MIK } \\
1.43\end{array}$ & $\begin{array}{l}\text { MIK } \\
0.86\end{array}$ & $\begin{array}{l}\text { MIK } \\
0.78\end{array}$ & $\begin{array}{l}\text { MIK } \\
0.71\end{array}$ & $\begin{array}{l}\text { MIK } \\
0.54\end{array}$ & $\begin{array}{l}\text { MIK } \\
0.45\end{array}$ \\
\hline Turbellaria & & & & & & \\
\hline $\begin{array}{l}\text { Planariidae } \\
\text { Planariidae? }\end{array}$ & $X^{a}$ & & $\mathbf{X}$ & & $\begin{array}{l}\mathbf{X} \\
\mathbf{X}\end{array}$ & \\
\hline Nematoda & $\mathbf{x}$ & $\mathbf{X}$ & $\mathbf{X}$ & & $\mathbf{X}$ & $\mathbf{X}$ \\
\hline Oligochaeta & $\mathbf{X}$ & $\mathbf{X}$ & $\mathbf{X}$ & $\mathbf{X}$ & $\mathbf{X}$ & $\mathbf{X}$ \\
\hline Crustacea & & & & & & \\
\hline $\begin{array}{l}\text { Copepoda } \\
\text { Ostracoda } \\
\text { Isopoda } \\
\text { Asellidae }\end{array}$ & $\mathbf{X}$ & $\mathbf{x}$ & $\begin{array}{l}\mathbf{X} \\
\mathbf{X}\end{array}$ & & & $\mathbf{X}$ \\
\hline $\begin{array}{c}\text { Asellus } \\
\text { Lirceus } \\
\text { Amphipoda } \\
\text { Gammaridae }\end{array}$ & $\begin{array}{l}\mathbf{X} \\
\mathbf{X}\end{array}$ & & & & & \\
\hline $\begin{array}{l}\text { Crangonyx } \\
\text { Decapoda }\end{array}$ & $\mathbf{X}$ & & & & & \\
\hline $\begin{array}{l}\text { Cambaridae } \\
\text { Hydracarina }\end{array}$ & $\begin{array}{l}\mathbf{X} \\
\mathbf{X}\end{array}$ & & & & & \\
\hline Hydracarina? & & & & & $\mathbf{X}$ & . \\
\hline Insecta & & & & & & \\
\hline Collembola & & & & & $\mathrm{X}$ & \\
\hline $\begin{array}{l}\text { Entomobryomorpha } \\
\text { Sminthuridae }\end{array}$ & $\begin{array}{l}\mathbf{X} \\
\mathbf{X}\end{array}$ & $\mathbf{X}$ & $\begin{array}{l}\mathbf{X} \\
\mathbf{X}\end{array}$ & $\mathbf{X}$ & $\mathbf{X}$ & $\begin{array}{l}X \\
X\end{array}$ \\
\hline $\begin{array}{l}\text { Ephemeroptera } \\
\text { Baetidae }\end{array}$ & & & & & & \\
\hline $\begin{array}{l}\text { Baetis } \\
\text { Cloeon }\end{array}$ & $\begin{array}{l}X \\
X\end{array}$ & $X$ & & & & \\
\hline Ephemerellidae & & & & & & \\
\hline $\begin{array}{l}\text { Eurylophella } \\
\text { Ephemeridae }\end{array}$ & X & & & & & \\
\hline Ephemera & $\mathbf{X}$ & & & & & \\
\hline $\begin{array}{l}\text { Ephemeridae? } \\
\text { Leptophlebiidae }\end{array}$ & $\begin{array}{l}X \\
X\end{array}$ & & & & & \\
\hline Habrophlebiodes & $\mathrm{X}$ & & & & & \\
\hline Habrophlebiodes? & $\mathrm{X}$ & & & & & \\
\hline
\end{tabular}


Table E1 (continued)

\begin{tabular}{|c|c|c|c|c|c|c|}
\hline \multirow[b]{2}{*}{ Taxon } & \multicolumn{6}{|c|}{ Site } \\
\hline & $\begin{array}{l}\text { MIK } \\
1.43\end{array}$ & $\begin{array}{l}\text { MIK } \\
0.86\end{array}$ & $\begin{array}{l}\text { MIK } \\
0.78\end{array}$ & $\begin{array}{l}\text { MIK } \\
0.71\end{array}$ & $\begin{array}{l}\text { MIK } \\
0.54\end{array}$ & $\begin{array}{l}\text { MIK } \\
0.45\end{array}$ \\
\hline \multicolumn{7}{|l|}{ Ephemeroptera (con.t) } \\
\hline Lepthophlebia & $\mathbf{X}$ & & & & & \\
\hline Paraleptophlebia & $\mathbf{x}$ & & & & & \\
\hline $\begin{array}{l}\text { Paraleptophlebia? } \\
\text { Jiphiōnuridae }\end{array}$ & $\mathbf{X}$ & & & & & \\
\hline Ameletus & $\mathbf{X}$ & & & & & \\
\hline \multicolumn{7}{|l|}{ Odonata } \\
\hline $\begin{array}{l}\text { Anisoptera } \\
\text { Aeshnidae }\end{array}$ & $\mathbf{x}$ & & & & & \\
\hline $\begin{array}{c}\text { Boyeria vinosa } \\
\text { Cordulegastridae }\end{array}$ & & & $\mathbf{x}$ & & & \\
\hline Cordulegaster & $\mathbf{x}$ & & & & & \\
\hline Cordulegaster? & $\mathbf{X}$ & & & & & \\
\hline Gomphidae & $\mathbf{X}$ & & & $\mathbf{X}$ & & \\
\hline Lanthus? & $\mathbf{X}$ & & & & & \\
\hline $\begin{array}{c}\text { Stylogomphus } \\
\text { albistylus }\end{array}$ & $\mathbf{X}$ & & & & & \\
\hline Libellula? & & & & & & $\mathbf{X}$ \\
\hline Libellulidae? & $\mathbf{X}$ & & & & & \\
\hline $\begin{array}{l}\text { Zygoptera } \\
\text { Calopterygidae }\end{array}$ & & & & $\mathbf{X}$ & & $\mathbf{X}$ \\
\hline Calopteryx & $\mathbf{X}$ & & $\mathbf{X}$ & & & \\
\hline Coenagrionidae & & & $\mathbf{X}$ & $\mathbf{X}$ & $\mathbf{X}$ & $\mathbf{X}$ \\
\hline Argia & & & $\mathbf{x}$ & $\mathbf{X}$ & $\mathbf{X}$ & $\mathbf{X}$ \\
\hline Enallagma & & & & & $\mathbf{X}$ & $\mathbf{X}$ \\
\hline Enallagma/Ischnura & & & $\mathbf{X}$ & & $\mathbf{X}$ & \\
\hline $\begin{array}{l}\text { Enallagma? } \\
\text { Ischnura? }\end{array}$ & & & & & & $\begin{array}{l}\mathbf{X} \\
\mathbf{X}\end{array}$ \\
\hline Coenagrionidae? & $\mathbf{X}$ & & & $\mathbf{X}$ & & \\
\hline Plecoptera & & & & & & \\
\hline Capniidae & & $\mathbf{X}$ & & & & \\
\hline $\begin{array}{l}\text { Allocapnia } \\
\text { Leuctridae }\end{array}$ & $\mathbf{X}$ & & & & & \\
\hline $\begin{array}{c}\text { Leuctra } \\
\text { Nemouridae }\end{array}$ & & $\mathbf{X}$ & & & & \\
\hline Amphinemura & $\mathbf{X}$ & & & & & \\
\hline Nemoura & & $\mathbf{x}$ & & & & \\
\hline Nemoura? & $\mathbf{X}$ & & & & & \\
\hline
\end{tabular}


Table E1 (continued)

\begin{tabular}{|c|c|c|c|c|c|c|}
\hline \multirow[b]{2}{*}{ Taxon } & \multicolumn{6}{|c|}{ Site } \\
\hline & $\begin{array}{l}\text { MIK } \\
1.43\end{array}$ & $\begin{array}{l}\text { MIK } \\
0.86\end{array}$ & $\begin{array}{l}\text { MIK } \\
0.78\end{array}$ & $\begin{array}{l}\text { MIK } \\
0.71\end{array}$ & $\begin{array}{l}\text { MIK } \\
0.54\end{array}$ & $\begin{array}{l}\overline{M I K} \\
0.45\end{array}$ \\
\hline \multicolumn{7}{|l|}{ Plecoptera (cont.) } \\
\hline Perlidae & & $\mathbf{X}$ & & & & \\
\hline Perlodidae & $\mathbf{x}$ & & & & & \\
\hline Clioperla clio & $\mathrm{x}$ & & & & & \\
\hline Isoperla? & $\mathbf{x}$ & & & & & \\
\hline Perlodidae? & $x$ & & & & & \\
\hline \multicolumn{7}{|l|}{ Hemiptera } \\
\hline \multicolumn{7}{|l|}{ Microveliidae } \\
\hline Microvelia & $\mathbf{X}$ & & & & & \\
\hline Microvelia? & & & & $\mathrm{x}$ & $\mathbf{x}$ & \\
\hline \multicolumn{7}{|l|}{ Notonectidae } \\
\hline Notonecta & & & & & $\mathbf{X}$ & \\
\hline \multicolumn{7}{|l|}{ Megaloptera } \\
\hline Corydalidae & $\mathbf{X}$ & & & & & \\
\hline \multicolumn{6}{|l|}{ Nipronia } & \\
\hline fasciatus & $\mathbf{x}$ & & & & & \\
\hline \multicolumn{7}{|l|}{ Sialidae } \\
\hline Sinlis & $\mathbf{X}$ & & & & & \\
\hline \multicolumn{7}{|l|}{ Trichoptera } \\
\hline $\begin{array}{c}\text { Hydropsychidae } \\
\text { Diplectrona }\end{array}$ & & & & & & $\mathbf{X}$ \\
\hline modesta & $\mathbf{X}$ & $\mathbf{X}$ & & & & \\
\hline Limnephilidae & $\mathrm{x}$ & & & & & \\
\hline Goera & $\mathbf{X}$ & & & & & . \\
\hline Neophylax & $\mathrm{X}$ & & & & & \\
\hline Pycnopsyche & $\mathrm{X}$ & & & & & \\
\hline \multicolumn{7}{|l|}{ Molannidae } \\
\hline Molanna & $\mathbf{X}$ & & & & & \\
\hline Phryganeidae? & & & & & & $\mathrm{X}$ \\
\hline \multicolumn{7}{|l|}{ Coleoptera } \\
\hline \multicolumn{7}{|l|}{ Dryopidae } \\
\hline Helichus & $\mathbf{X}$ & & & & & \\
\hline Dytiscidae? & & & & & & $\mathrm{X}$ \\
\hline Elmidae & $\mathbf{X}$ & & & & & \\
\hline Dubiraphia & $\mathrm{X}$ & & & & & \\
\hline Optioservus & $\mathrm{X}$ & $\mathbf{X}$ & $\mathbf{X}$ & & & $\mathbf{X}$ \\
\hline Stenelmis & $\mathrm{X}$ & $\mathbf{X}$ & $\mathrm{X}$ & $\mathbf{x}$ & & \\
\hline
\end{tabular}


Table E.1 (continued)

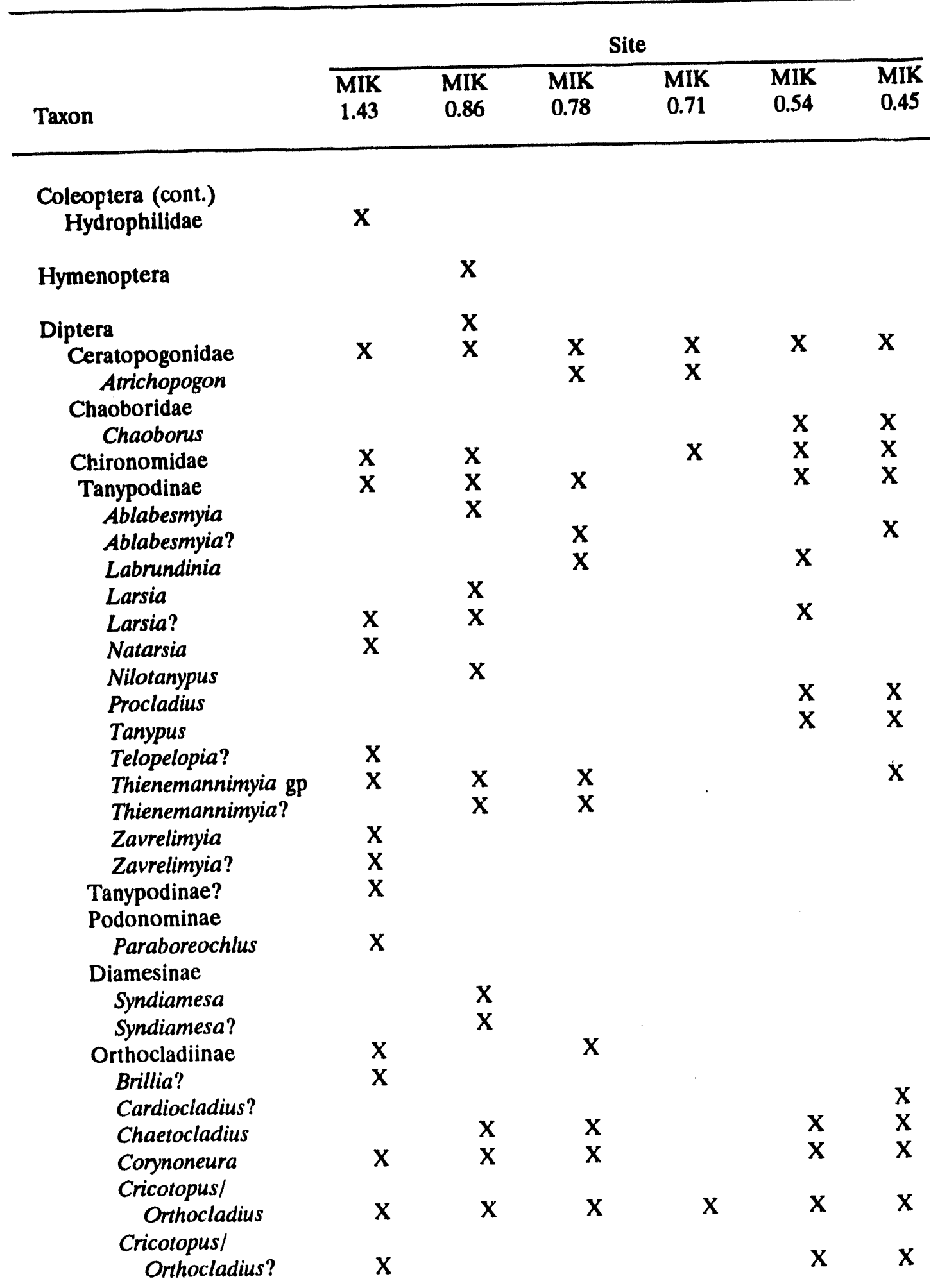


Table E.1 (continued)

\begin{tabular}{|c|c|c|c|c|c|c|}
\hline \multirow[b]{2}{*}{ Taxon } & \multicolumn{6}{|c|}{ Site } \\
\hline & $\begin{array}{l}\text { MIK } \\
1.43\end{array}$ & $\begin{array}{l}\text { MIK } \\
0.86\end{array}$ & $\begin{array}{l}\text { MIK } \\
0.78\end{array}$ & $\begin{array}{l}\text { MIK } \\
0.71\end{array}$ & $\begin{array}{l}\text { MIK } \\
0.54\end{array}$ & $\begin{array}{l}\text { MIK } \\
0.45\end{array}$ \\
\hline Chironomidae (cont.) & & & & & & \\
\hline Diplocladius & $\mathbf{X}$ & & & & & \\
\hline Gymnometriocnemus? & & & & & $\mathbf{x}$ & \\
\hline $\begin{array}{l}\text { Heleniella } \\
\text { Heterotrissocladius }\end{array}$ & $\begin{array}{l}\mathbf{X} \\
\mathbf{X}\end{array}$ & & & & & \\
\hline Limnophyes & & & $\mathbf{X}$ & & & \\
\hline Limnophyes? & & & $\mathbf{X}$ & & $\mathbf{X}$ & \\
\hline Nanocladius? & $\mathrm{X}$ & & & & & \\
\hline $\begin{array}{l}\text { Parakiefferiella? } \\
\text { Parametriocnemus }\end{array}$ & $\mathrm{x}$ & $\mathbf{X}$ & & $\mathbf{X}$ & & $\mathbf{X}$ \\
\hline Parametriocnemus? & $\mathbf{X}$ & & & & & $\mathbf{x}$ \\
\hline Psilometriocnemus? & $X$ & & & & & \\
\hline Rheocricotopus? & $\mathbf{X}$ & $\mathbf{X}$ & & & & Y \\
\hline Thienemanniella & & & & & & \\
\hline Orthocladiinae? & $\begin{array}{l}\mathbf{X} \\
\mathbf{X}\end{array}$ & & & & & \\
\hline Chironominae & $\mathbf{X}$ & & & & $\mathbf{X}$ & $\mathbf{X}$ \\
\hline Chironomini & & & & & & \\
\hline Chironomus & & & $\mathbf{X}$ & & $\mathbf{X}$ & $\mathrm{X}$ \\
\hline Chironomus/ & & & & & & \\
\hline Goeldichironomus & & & & & $\mathbf{X}$ & \\
\hline Chironomus? & & & $\mathbf{X}$ & $\mathbf{X}$ & $\mathbf{X}$ & \\
\hline Cryptochironomus & $X$ & & & & $\mathrm{X}$ & $\mathbf{X}$ \\
\hline Cryptotendipes & $\mathbf{X}$ & & & & & \\
\hline Cryptotendipes? & $\mathbf{X}$ & & & & & \\
\hline Dicrotendipes & & & & & $\mathrm{X}$ & $X$ \\
\hline Endochironomus & $\mathbf{X}$ & & & & & \\
\hline Endochironomus? & $\mathbf{X}$ & & & & & \\
\hline Glyptotendipes & & & & $\mathbf{X}$ & $\mathbf{X}$ & $\mathrm{X}$ \\
\hline Goeldichironomus & & $\mathrm{X}$ & & & $\mathrm{X}$ & $\mathrm{X}$ \\
\hline Goeldichironomus? & $\mathbf{X}$ & & & & $\mathrm{X}$ & \\
\hline Microtendipes & $\mathrm{X}$ & $\mathbf{X}$ & $\mathrm{X}$ & & $\mathrm{X}$ & $\mathbf{X}$ \\
\hline Microtendipes? & & & & $\mathbf{X}$ & & \\
\hline Paralauterborniella & $\mathbf{X}$ & & & & & \\
\hline Paralauterborniella? & $\mathrm{X}$ & & & & & \\
\hline Paratendipes & $\mathbf{X}$ & $\mathbf{X}$ & & & & \\
\hline Phaenopsectra & $\mathbf{X}$ & & & & & \\
\hline Polypedilum & $\mathbf{X}$ & $\mathrm{X}$ & $\mathrm{X}$ & $\mathbf{X}$ & & \\
\hline Stictochironomus & $\mathbf{X}$ & & $\mathrm{X}$ & & $X$ & \\
\hline Chironominae? & $\mathbf{X}$ & & $\mathrm{X}$ & & & \\
\hline
\end{tabular}


Table E.1 (continued)

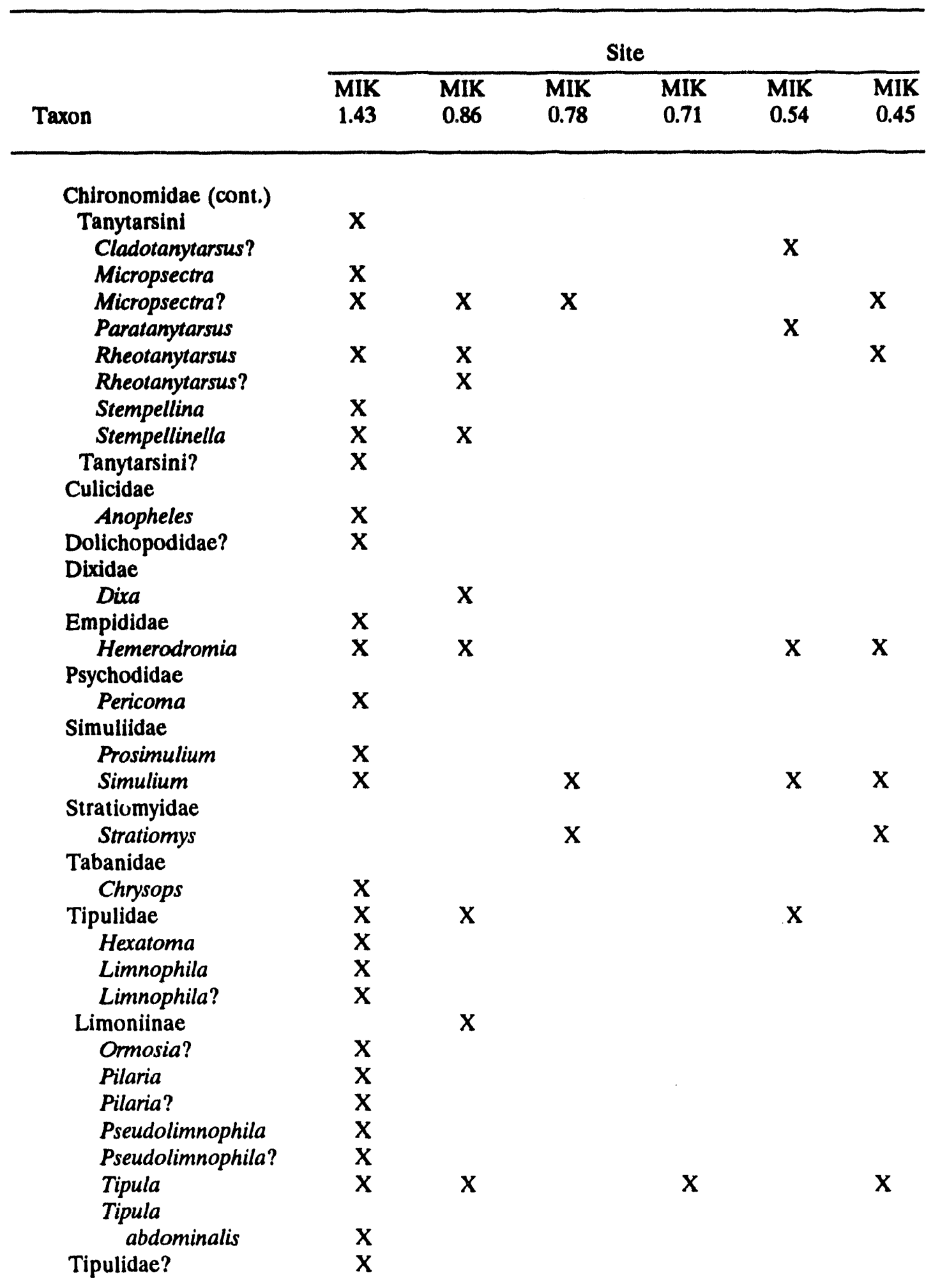


Tat le E.1 (continued)

\begin{tabular}{|c|c|c|c|c|c|c|}
\hline \multirow[b]{2}{*}{ Taxon } & \multicolumn{6}{|c|}{ Site } \\
\hline & $\begin{array}{l}\text { MIK } \\
1.43\end{array}$ & $\begin{array}{l}\text { MIK } \\
0.86\end{array}$ & $\begin{array}{l}\text { MIK } \\
0.78\end{array}$ & $\begin{array}{l}\text { MIK } \\
0.71\end{array}$ & $\begin{array}{l}\text { MIK } \\
0.54\end{array}$ & $\begin{array}{l}\text { MIK } \\
0.45\end{array}$ \\
\hline \multicolumn{7}{|l|}{ Mollusca } \\
\hline $\begin{array}{l}\text { Gastropoda } \\
\text { Ancylidae }\end{array}$ & $\mathbf{X}$ & & & & $\mathbf{X}$ & \\
\hline $\begin{array}{r}\text { Ferrissia } \\
\text { Lymnaeidae }\end{array}$ & $\mathbf{X}$ & & & & & $\mathbf{X}$ \\
\hline $\begin{array}{l}\text { Lymnaeidae? } \\
\text { Physidae }\end{array}$ & & & $\mathbf{X}$ & & & $\mathrm{X}$ \\
\hline $\begin{array}{l}\text { Physella } \\
\text { Bivalvia }\end{array}$ & & & $\mathbf{X}$ & & & \\
\hline Sphaeriidae & $\mathbf{x}$ & & & & & \\
\hline Pisidium & $\mathbf{x}$ & & & & & \\
\hline Pisidium? & $\mathbf{X}$ & & & & & \\
\hline Sphaerium & $\mathbf{X}$ & & & & & \\
\hline
\end{tabular}

"An "X" denotes that the taxon was collected at least once in quantitative samples. Note: MIK = Mitchell Branch kilometer. 
ORNL/TM-12150

\section{INTERNAL DISTRIBUTION}

1. S. M. Adams

2. L. J. Allison

3. T. L. Ashwood

4. B. G. Blaylock

5. E. B. Bryant

6. C. F. Cada

7. S. W. Christensen

8. M. L. Coffey

9. R. B. Cook

10. C. C. Coutant

11. C. E. Duncan

12-14. J. M. Forstrom

15. R. E. Frounfelker

16. C. W. Gehrs

17. M. S. Greeley

18. S. G. Hildebrand

19-28. R. L. Hinzman

29. M. A. Huston

30. L. J. Jennings

31. J. A. Kovacs

32-41. L. A. Kszos

42. K. L. Lee

43-47. J. M. Loar

48. R. J. Olson

49. M. J. Peterson
50. R. Petrie

51. A. S. Quist

52. R. A. Rich

53-57. J. L. Rich

58. C. D. Runk

59. M. G. Ryon

60. E. M. Schilling

61. J. A. Shaakir-Ali

62. L. R. Shugart

63-65. J. G. Smith

66. G. R. Southworth

67. A. J. Stewart

68. G. W. Suter

69. M. Swearingen

70. S. S. Talmage

71. V. R. Tolbert

72. W. Van Winkle

73. Central Research Library

74. EMD Document Center

75-89. ESD Library

90. ER Document Management Center

91-92. Laboratory Records Department

93. Laboratory Records, ORNL-RC

94. K-25 Site Records Department

95. ORNL Patent Office

\section{EXTERNAL DISTRIBUTION}

96. S. Ahlstedt, Division of Water Resources, Tennessee Valley Authority, Norris, TN 37828

97. D. L. Buhaly, Environmental Operation Branch, Environmental Protection Division, SE-31, Department of Energy Oak Ridge Operations, P.O. 2001, Oak Ridge, TN 37831

98. L. Chang, U.S. Environmental Protection Agency, 26 W. Martin Luther King Drive, Cincinnati, OH 45268

99. B. Cook, Tennessee Department of Environment and Conservation, DOE Oversight Division, 761 Emory Valley Road, Oak Ridge, TN 37830

100. P. H. Edmonds, Radian Corporation, 120 South Jefferson Circle, Oak Ridge, TN 37830

101. M. T. Fagg, Tennessee Wildlife Resources Agency, Ellington Agricultural Center, P.O. Box 40747, Nashville, TN 37204

102. R. N. Farvolden, Professor, Department of Earth Sciences, University of Waterloo, Waterloo, Ontario N2L 3G1 CANADA 

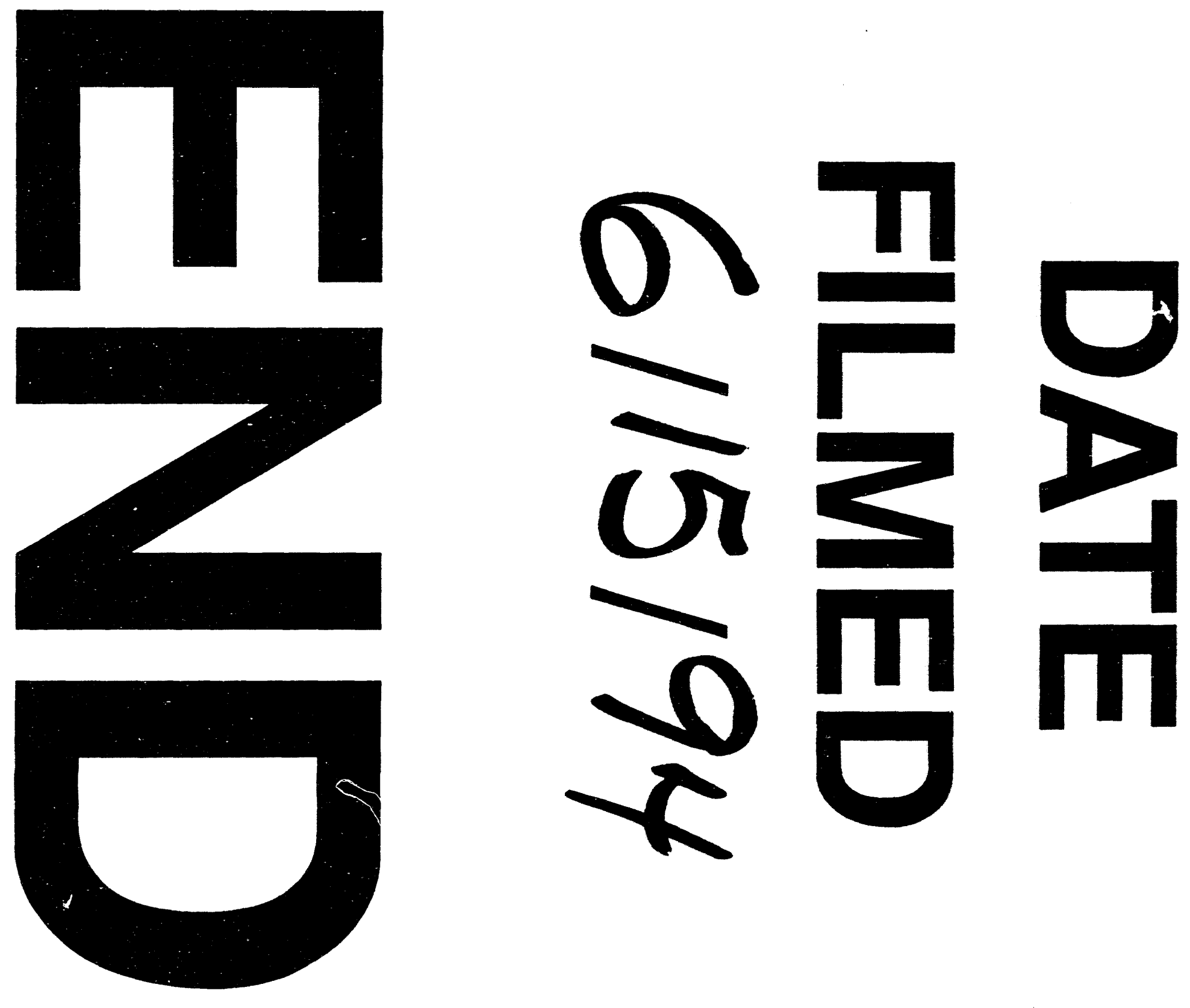


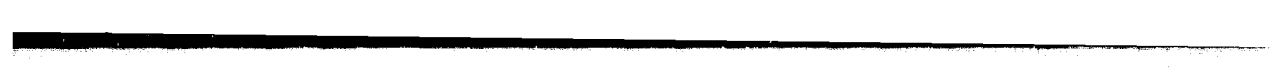

